\title{
Weaning-induced alterations on neuropeptidergic populations of the rat hypothalamus
}

Citation for published version (APA):

Diniz, G. B. (2018). Weaning-induced alterations on neuropeptidergic populations of the rat hypothalamus. [Doctoral Thesis, Maastricht University]. Maastricht University. https://doi.org/10.26481/dis.20181218gd

Document status and date:

Published: 01/01/2018

DOI:

10.26481/dis.20181218gd

Document Version:

Publisher's PDF, also known as Version of record

\section{Please check the document version of this publication:}

- A submitted manuscript is the version of the article upon submission and before peer-review. There can be important differences between the submitted version and the official published version of record. People interested in the research are advised to contact the author for the final version of the publication, or visit the DOI to the publisher's website.

- The final author version and the galley proof are versions of the publication after peer review.

- The final published version features the final layout of the paper including the volume, issue and page numbers.

Link to publication

\footnotetext{
General rights rights.

- You may freely distribute the URL identifying the publication in the public portal. please follow below link for the End User Agreement:

www.umlib.nl/taverne-license

Take down policy

If you believe that this document breaches copyright please contact us at:

repository@maastrichtuniversity.nl

providing details and we will investigate your claim.
}

Copyright and moral rights for the publications made accessible in the public portal are retained by the authors and/or other copyright owners and it is a condition of accessing publications that users recognise and abide by the legal requirements associated with these

- Users may download and print one copy of any publication from the public portal for the purpose of private study or research.

- You may not further distribute the material or use it for any profit-making activity or commercial gain

If the publication is distributed under the terms of Article $25 \mathrm{fa}$ of the Dutch Copyright Act, indicated by the "Taverne" license above, 


\section{Weaning-induced alterations on neuropeptidergic populations of the rat hypothalamus}

\section{Dissertation}

To obtain the degree of Doctor at Maastricht University, on the authority of the Rector Magnificus,

Prof. Dr. Rianne M. Letschert

in accordance with the decision of the Board of Deans, to be defended in public on Tuesday $18 / 12 / 2018$, at 10:45 hours

by

Giovanne B. Diniz 


\section{Supervisor:}

Prof. Dr. Harry W. M. Steinbusch

\section{Co-Supervisor:}

Prof. Dr. Jackson C. Bittencourt University of São Paulo, Brazil

\section{Assessment Committee:}

Prof. Dr. B. Joosten (Chairman)

Dr. C. F. Elias University of Michigan, USA

Prof. Dr. L. Köhler

Prof. Dr. T. Kozicz Mayo Clinic, Rochester, USA

Dr. F. van Leeuwen

\section{Financial Support:}

The experimental investigations and the writing of this Dissertation would not be possible without financial aid from the São Paulo Research Foundation (FAPESP), grants \#2016/02748-0, \#2016/02224-1 and \#2017/08908-2. Additional support was provided by the Brazilian Coordination for the Improvement of Higher Education Personnel (CAPES), grant CAPES/COFECUB \#848/15, and by the Brazilian National Council for Scientific and Technological Development (CNPq), grant \#426378/2016-4. 


\section{List of Abbreviations}

-ir -immunoreactive

AgRP Agouti-related neuropeptide

CNS Central nervous system

Fos Proto-oncogene c-Fos

FSH Follicle-stimulating hormone

GABA $\gamma$-aminobutyric acid

GnRH Gonadotropin-releasing hormone

Hcrt Hypocretin neuropeptide precursor (gene)

IHy Incerto-hypothalamic area

LH Luteinizing hormone

LHA Lateral hypothalamic area

MCH Melanin-concentrating hormone

MCHR1 Melanin-concentrating hormone receptor 1

Mchr1 Melanin-concentrating hormone receptor 1 (gene)

MCHR2 Melanin-concentrating hormone receptor 2

MPOA Medial preoptic area of the hypothalamus

NPY Neuropeptide Y

ORX Orexins

Pmch Pro-melanin-concentrating hormone (gene)

a-MSH a-melanocyte-stimulating hormone 



\section{Table of Contents}

\section{Chapter 1}

General Introduction.

.4

\section{Chapter 2}

Neuroanatomical Structure of the MCH System....

\section{Chapter 3}

The Melanin-Concentrating Hormone as an Integrative Peptide Driving Motivated Behaviors

\section{Chapter 4}

Litter size determines the number of melanin-concentrating hormone neurons in the medial preoptic area of Sprague-Dawley lactating dams.

\section{Chapter 5}

Suckling-induced Fos activation and melanin-concentrating hormone immunoreactivity during late lactation.

\section{Chapter 6}

The weaning period promotes alterations in the orexin neuronal population of rats in a suckling-

dependent manner....

\section{Chapter 7}

General Discussion

\section{Appendix A}

Summary [English]

\section{Appendix B}

Summary [Dutch].

\section{Appendix C}

Valorisation...

\section{Appendix D}

About the Author.

\section{Appendix E}

Acknowledgements. 

General Introduction 



\subsection{Lactation and Weaning}

The theory of evolution by natural selection, by Charles R. Darwin, can be simplified in three main principles: variation, heredity and selection. These principles ensure that variations acquired by one individual that are positively selected by the environment may be perpetuated through the offspring of that individual. Several animal species, however, evolved to be sexually immature at birth, requiring a development time before they are able to generate their own progeny. Because death of the developing offspring before sexual maturation incurs in the loss of that particular set of genes, making it a powerful negative selector, several species were positively selected for developing strategies that maximize the survival of their progeny. This resulted in the development of a myriad of reproductive strategies, including lactation, the hallmark feature of the Mammalia class.

Lactation is the process by which the young receive their afterbirth nutrition directly from the mother through a secreted substance, milk. Since the offspring's nourishment comes straight from the mother's reserves, the dam can increase her energy stores during gestation in anticipation of the postpartum period. This is achieved through a series of concerted physiological alterations, which include increased intestinal villus height in the small intestine and colon expansion (Cripps and Williams 1975), increased fat storage (Naismith et al. 1982), steroidal hormone alterations (Morishige et al. 1973), and an increase in the ingestions of both food and liquid (Atherton et al. 1982; Cripps and Williams 1975; Shirley 1984). Nutrition, however, is but one aspect of lactation for most species with altricial progeny, such as lab rodents and humans. In those species, the dam must also supply the pups with other inputs, such as help to secrete, warmth, sensorial stimulation, play and social organization. Not supplying the litter can lead to long-term development problems (Liu et al. 2000).

One of the distinctive features of the lactation period is its complex temporal dynamics, since the needs of the litter change as the pups develop. Rats provide an excellent example of that dynamicity (Cramer et al. 1990; Thiels et al. 1990). On the first day of life, rat pups are virtually immobile, blind, deaf, unable to keep their own temperature or to secrete without help. The opening of the ear channel occurs by day four of life, and by the end of the first week of life the pups are able to crawl limited distances. By the end of the second week of life, however, the pups are able to see and walk without assistance, and the emergence of the incisor at day 8 allows then to forage and consume solid food (Altman and Sudarshan 1975; Smart and Dobbing 1971). The third week of life of the pups correspond to the period of weaning, as the pups become more reliant on solid food and spend less time on the nest (Thiels et al. 1990). By the end of the third week, the pups are independent and can be separated from the mother, as is the usual laboratory practice.

The weaning is an important time for the mother physiology, as the metabolic pressure exerted by the litter is progressively alleviated. This period allows the dam to prepare to resume sexual functions and to reset her homeostatic baselines to pre-gestational levels. To cope with such extensive alterations in a short span of time, several concerted brain alterations occur, many of them centered on the medial preoptic area of the hypothalamus (MPOA), a region necessary for the onset and maintenance of maternal behavior (Numan 1974; Numan et al. 1977). Since the alterations in behavior and physiology are so extensive, it is expected that other brain areas must also change in order to ensure proper energy homeostasis and awareness. In this process, the hypothalamus plays a major role. 


\subsection{The Parental Hypothalamus}

The hypothalamus, located deep in the forebrain and corresponding to only $0.2 \%$ of the total human brain mass, is one of the best conserved neural structures. A hypothalamus, or a homologous structure, can be found even in some of the most phylogenetically distant animals, such as lancelets (Moret et al. 2004). Since its development, the hypothalamus has been fundamentally conserved for its role in the maintenance of body homeostasis. This cardinal role cannot be executed without an enormous associated complexity, as each organism will find diverse environments and will have to respond appropriately to each one of them. It is natural, therefore, that animals with a more robust hypothalamus, capable of larger spectrum of responses, will have a fitness advantage against their peers, what resulted in a remarkable development in hypothalamus complexity regarding its spatial organization, connectivity, and chemical composition.

The hypothalamus has been implicated on the modulation of several physiological functions. Lesions of the hypothalamus lead to eating disorders (Anand and Brobeck 1951), loss of osmotic regulation and circadian pace keeping (Stephan and Zucker 1972), increase in thresholds of gustatory preference-aversion (Ferssiwi et al. 1987), alteration of basal temperature (Satinoff and Shan 1971), deficit of reproductive behavior (Pfaff and Sakuma 1979) and precocious puberty (Donovan and Ten Bosch 1956), sleep disorganization (Lin et al. 1989), changes in autonomic activity (Yoshimatsu et al. 1984) and disturbances in hormone secretion (Bernardis and Frohman 1970), to cite a few. The deleterious effects following discreet hypothalamic lesions exemplify well the fundamental actions played by hypothalamic neurons, especially on the maintenance of the homeostatic balance.

During pregnancy and lactation, several homeostatic baselines must be reset to accommodate the new temporary needs of the mother (Brunton and Russell 2008). Since the fat storages of the female increase several-fold during pregnancy, the hypothalamus became less sensitive to the anorectic effects of leptin (Seeber et al. 2002; Trujillo et al. 2011). To promote that increase, the arcuate nucleus sees an increase in its orexigenic population (NPY/AgRP) while the anorectic effects of a-MSH are abolished (Augustine et al. 2008). To allow the increase in blood volume necessary for placental maintenance, vasopressinergic neurons of the paraventricular nucleus have their secretion threshold reduced by placental hormones (Sunn et al. 2002). Tuberoinfundibular dopaminergic neurons are suppressed before lactation, allowing a surge in prolactin synthesis by the anterior hypophysis (Brunton and Russell 2008). The stress responses of the female are attenuated during pregnancy, in a mechanism that involves endogenous opioids (Atkinson and Waddell 1995; Brunton et al. 2005). The supraoptic and paraventricular hypothalamic nuclei undergo morphological alterations, with glial processes retreating to allow widespread communication between oxytocinergic neurons, necessary for milk letdown during lactation (Theodosis 2002). The medial preoptic area is recruited by prolactin and estrogen and organizes the circuit that will allow the mother to replace an innate aversive response to the pups for the wellcharacterized maternal behavior that will ensure the litter survival (Morgan et al. 1999; Numan and Stolzenberg 2009).

Contrasting to the well-known alterations that take place in the medial and periventricular zones of the hypothalamus, which includes the MPOA, paraventricular, supraoptic and arcuate nuclei, less is known about the role of the hypothalamic lateral zone in pregnancy and lactation. In the current scheme of parcellation employed by most atlases, approximately all of the hypothalamus comprehended 
between the fornix and the internal capsule and between the optic chiasm and the mammillary bodies is considered part of the lateral hypothalamic area (LHA), closely following the definition of lateral hypothalamus provided by Gurdjian (1927). The LHA is also known as the bed nucleus of the medial forebrain bundle, arguably the largest rostrocaudal tract of fibers in the prosencephalon (Nieuwenhuys et al. 1982; Veening et al. 1982), as fibers from this bundle are found intermingled with LHA neurons (Geeraedts et al. 1990). This confers a remarkable connectivity to the LHA, rivaled only by its varied neurochemical composition. Among the neuromodulators synthesized in this area we find the melaninconcentrating hormone $(\mathrm{MCH})$ and the orexins (ORX), intermingled with a vast population of GABAergic and glutamatergic interneurons (Bittencourt et al. 1992; Gao and van den Pol 2000; Peyron et al. 1998). These peptides compose the neurochemical signature of two different populations that reside in the LHA, since MCH neurons do not synthesize ORX and vice-versa (Elias et al. 1998; Peyron et al. 1998).

\subsection{Melanin-concentrating hormone}

Mammalian MCH is a nonadecapeptide with a cyclic structure found in virtually all animals belonging to this phylum (Nahon et al. 1989; Vaughan et al. 1989). The MCH-coding gene, called Pmch, shows remarkable conservation when compared to its homologue in a cartilaginous fish (Sphyrna lewini), suggesting it has been maintained under considerable evolutive pressure along vertebrate evolution. This conservation reflects the fundamental roles played by MCH in mammalian physiology. Those roles are supported by a complex distribution of $\mathrm{MCH}$-immunoreactive ( $\mathrm{MCH}$-ir) neurons, which can be found in several subcortical structures, with a particularly high concentration in the LHA. Projections immunoreactive to $\mathrm{MCH}$ can be found widespread throughout the central nervous system, allowing MCH neurons to interact with several different brain circuits and neurochemical populations (Bittencourt et al. 1992).

Melanin-concentrating hormone has been associated with over 12 families of functions, including ingestive behavior (Ludwig et al. 2001; Qu et al. 1996; Shimada et al. 1998), energy expenditure (Kennedy et al. 2001; Segal-Lieberman et al. 2003; Verty et al. 2010), sleep architecture (Lagos et al. 2011; Tsunematsu et al. 2014; Verret et al. 2003), mood modulation (Borowsky et al. 2002; Georgescu et al. 2005; Monzon et al. 2001), stress response (Kennedy et al. 2003; Smith et al. 2006), sexual physiology (Gonzalez et al. 1996; Murray et al. 2000; Murray et al. 2006; Naufahu et al. 2013), maternal behavior (Knollema et al. 1992; Rondini et al. 2010), learning and memory (Adamantidis et al. 2005; Monzon and De Barioglio 1999; Pachoud et al. 2010), ventricular homeostasis (Conductier et al. 2013a; Conductier et al. 2013b), autonomic modulation (Egwuenu et al. 2012; Messina and Overton 2007), immune response (Coumans et al. 2007; Kokkotou et al. 2009) and sensory integration (Adams et al. 2011; Miller et al. 1993). Several of the functions associated to $\mathrm{MCH}$ are altered during pregnancy and lactation, suggesting it is very unlikely that $\mathrm{MCH}$ does not mediate at least some of the parental brain alterations necessary to maximize the litter survival. The precise relationship between $\mathrm{MCH}$ and lactation, however, has been hard to pinpoint.

The first suggestion of an $\mathrm{MCH}$ action on the lactating brain comes from the work of Knollema et al. (1992), which found expression of Pmch and synthesis of the peptide in the MPOA, periventricular preoptic nucleus and anterior paraventricular nucleus exclusively during the late lactation period. This expression/synthesis is exclusive to dams, with no signal detected during gestation, early lactation or after the weaning of the pups. The proximity between $\mathrm{MCH}$ and oxytocin-synthesizing neurons of the 
paraventricular nucleus and of $\mathrm{MCH}$-ir fibers to oxytocin-ir fibers in the median eminence led those authors to speculate a role in hormonal secretion, a possibility further investigated by Parkes and Vale (1993). The application of MCH to collected posterior pituitaries increased the amount of oxytocin in the collected medium, suggesting MCH acts at the level of the pituitary to promote the release of this hormone, thereby contributing to the milk ejection-reflex during lactation.

Despite the initial development in linking $\mathrm{MCH}$ and lactation, the field went then relatively quiescent for over twenty years, until Benedetto et al. (2014) described that exogenous MCH application in the MPOA decreases the active components of maternal behavior. Considering that Pmch is increasingly expressed in the MPOA as lactation progresses (Knollema et al. 1992; Rondini et al. 2010), that study was the first to provide compelling evidence that $\mathrm{MCH}$ is part of the brain alterations that take place during weaning to restore the maternal brain to its non-lactating baseline and interrupt the pupdirected behavior. Although evidence started to build up for an $\mathrm{MCH}$ role in neuroendocrine control and maternal behavior, it is still unclear if $\mathrm{MCH}$ plays a role in the metabolic aspect of lactation, given its important action on energy balance in other physiological states. Furthermore, the signals that inform the MCH peptidergic system of the progress of lactation are still unknown, as is the relationship between $\mathrm{MCH}$ and weaning. This knowledge may help us better understand how MCH neurons connect to a broader lactation network.

\subsection{Orexins}

The ORX peptidergic system is formed by two peptides, called orexin A and orexin B, also known as hypocretin-1 and hypocretin-2 (de Lecea et al. 1998; Sakurai et al. 1998). These two peptides originate from a single preprohormone, coded by the Hcrt gene. Orexin A is a cyclic peptide with 33 amino acids, while orexin B is a linear peptide with 28 amino acids. Despite the structural disparity, both orexins act on the same set of receptors with comparable effects. As is the case of $\mathrm{MCH}$, the amino acid sequences of the orexins are also highly conserved among vertebrate species (Tsujino and Sakurai 2009).

In the rat, ORX-ir neurons are found exclusively in the LHA, with the highest number of neurons in the perifornical nucleus, although a continuous of neurons is found from the medial hypothalamus to the laterodorsal LHA and the posterior hypothalamic area (Date et al. 1999; Nambu et al. 1999; Peyron et al. 1998). Orexin-immunoreactive fibers are found in several subcortical areas of the CNS but are seldom found in cortical fields. The densest areas of ORX innervation are, in the rat, the paraventricular thalamic nucleus, arcuate nucleus, raphe nuclei, the dorsal tuberomammillary nucleus and the locus coeruleus (Date et al. 1999; Nambu et al. 1999; Peyron et al. 1998). There is no difference between the staining pattern of an antibody selective for ORXA and one that stains both ORXA and ORXB, suggesting a high degree of colocalization between these two peptides (Peyron et al. 1998).

The ample distribution of ORX-ir fibers in subcortical areas of the mammalian brain is reflective of the numerous functions performed by ORX-synthesizing neurons. The most well-known ORX action is on the maintenance of sleep architecture, as animals with deletion of the Hcrt gene display a behavior similar to narcolepsy, with impromptu bouts of sleep during the active time (Chemelli et al. 1999; Hara et al. 2001; Lin et al. 1999). The orexins have also been associated to arousal, attention and behavioral responses to stress (Furlong et al. 2009; Mahler et al. 2014), reward and hedonic responses (Harris and Aston-Jones 2006; Mahler and Aston-Jones 2012) and energy expenditure (Hagan et al. 1999; Wang et 
al. 2001). Further reinforcing the similarities between the MCH and the ORX peptidergic systems, the orexins were also associated to a potent orexigenic action (Edwards et al. 1999; Haynes et al. 1999; Sakurai et al. 1998; Volkoff et al. 1999), although more recent works suggest the actions of ORX on feeding may be subtler and depend on the physiological state of the animal (González et al. 2016).

Regardless of the exact effect of ORX on feeding behavior, it is well-established that the orexins play an important role in energy homeostasis. Therefore, the ORX peptidergic system is a prime candidate to be modified during the new brain status quo of the lactating female. Previous studies, however, failed to reach a consensus about the exact role of orexins during lactation (Brogan et al. 2000; Cai et al. 2001; España et al. 2004; Garcia et al. 2003; Sun et al. 2003; Wang et al. 2003). Even more lackluster is the body of information available on the participation of orexin neurons in weaning and the re-establishing of the normal female brain.

\subsection{Aims and Outline of this Thesis}

Given the importance of lactation to mammalian species, the magnitude of transformations that take place in the maternal brain, and our lack of knowledge about the weaning period and the processes that underlie the return of the lactating brain to the resting state, we proposed with this work to investigate the influence of the suckling stimulus on the lateral hypothalamus during weaning, with special attention to the MCH and ORX populations.

In Chapter 2, we review the major morphological aspects of the MCH system, including all peptides synthesized from the MCH precursor, and its receptors. The neuroanatomy of the system is evaluated in depth, including detailed descriptions of the immunoreactive cell bodies, fibers and receptors in the mammalian brain. The need for studies in new animal models is highlighted.

In Chapter 3 we review the major functions associated to MCH and the underlying connectivity. We propose in this review that most $\mathrm{MCH}$ functions can be understood as a single process: the maintenance of the homeostatic baseline by $\mathrm{MCH}$, which works as a dampener to prevent possibly deleterious fluctuations in metabolic parameters.

In Chapter 4, we investigate the effect of litter size on the MPOA population of MCH neurons. To that end, mothers were separated in two groups according to the size of their naturally-occurring litter and the number of $\mathrm{MCH}$-ir neurons was evaluated at mid-lactation, early weaning and late weaning. Artificial culling of the litter after birth confirmed our results.

In Chapter 5, we investigate the acute effects of the suckling stimulus on the activation of MCH neurons in the whole hypothalamus of weaning rat dams. To suppress the suckling stimulus, a group of pups had their vibrissae pads anesthetized and were allowed to return to the home cage. The brain of the dams was then examined for the presence of the Fos protein in addition to MCH immunoreactivity.

In Chapter 6, we investigate if the Fos immunoreactivity in the tuberal hypothalamus of weaning dams found in the previous chapter is indicative of the recruitment of the orexin neuronal population present in this area. Three-dimensional reconstructions were employed to answer that question.

In Chapter 7, we discuss the main results found in the aforementioned works, with a focus on the perspectives in the field. 


\subsection{References}

Adamantidis A et al. (2005) Disrupting the melanin-concentrating hormone receptor 1 in mice leads to cognitive deficits and alterations of NMDA receptor function Eur J Neurosci 21:2837-2844

Adams AC, Domouzoglou EM, Chee MJ, Segal-Lieberman G, Pissios P, Maratos-Flier E (2011) Ablation of the hypothalamic neuropeptide melanin concentrating hormone is associated with behavioral abnormalities that reflect impaired olfactory integration Behav Brain Res 224:195-200

Altman J, Sudarshan K (1975) Postnatal development of locomotion in the laboratory rat Animal behaviour 23:896-920

Anand BK, Brobeck JR (1951) Hypothalamic control of food intake in rats and cats The Yale journal of biology and medicine 24:123

Atherton J, Dark J, Garland H, Morgan M, Pidgeon J, Soni S (1982) Changes in water and electrolyte balance, plasma volume and composition during pregnancy in the rat The Journal of physiology 330:81-93

Atkinson HC, Waddell BJ (1995) The hypothalamic-pituitary-adrenal axis in rat pregnancy and lactation: circadian variation and interrelationship of plasma adrenocorticotropin and corticosterone Endocrinology 136:512-520

Augustine RA, Ladyman SR, Grattan DR (2008) From feeding one to feeding many: hormone-induced changes in bodyweight homeostasis during pregnancy The Journal of physiology 586:387-397

Benedetto L, Pereira M, Ferreira A, Torterolo P (2014) Melanin-concentrating hormone in the medial preoptic area reduces active components of maternal behavior in rats Peptides 58:20-25

Bernardis LL, Frohman LA (1970) Effect of lesion size in the ventromedial hypothalamus on growth hormone and insulin levels in weanling rats Neuroendocrinology 6:319-328

Bittencourt JC et al. (1992) The melanin-concentrating hormone system of the rat brain: an immuno- and hybridization histochemical characterization J Comp Neurol 319:218-245

Borowsky B et al. (2002) Antidepressant, anxiolytic and anorectic effects of a melanin-concentrating hormone-1 receptor antagonist Nat Med 8:825-830

Brogan R, Grove K, Smith MS (2000) Differential regulation of leptin receptor but not orexin in the hypothalamus of the lactating rat Journal of neuroendocrinology 12:1077-1086

Brunton PJ, Meddle SL, Ma S, Ochedalski T, Douglas AJ, Russell JA (2005) Endogenous opioids and attenuated hypothalamicpituitary-adrenal axis responses to immune challenge in pregnant rats Journal of Neuroscience 25:5117-5126

Brunton PJ, Russell JA (2008) The expectant brain: adapting for motherhood Nature Reviews Neuroscience 9:11

Cai XJ, Denis R, Vernon RG, Clapham JC, Wilson S, Arch JR, Williams G (2001) Food restriction selectively increases hypothalamic orexin-B levels in lactating rats Regulatory peptides 97:163-168

Chemelli RM et al. (1999) Narcolepsy in orexin knockout mice: molecular genetics of sleep regulation Cell 98:437-451

Conductier $\mathrm{G}$ et al. (2013a) Melanin-concentrating hormone regulates beat frequency of ependymal cilia and ventricular volume Nature neuroscience 16:845-847

Conductier $\mathrm{G}$ et al. (2013b) Control of ventricular ciliary beating by the melanin concentrating hormone-expressing neurons of the lateral hypothalamus: a functional imaging survey Frontiers in endocrinology 4:182

Coumans B, Grisar T, Nahon JL, Lakaye B (2007) Effect of ppMCH derived peptides on PBMC proliferation and cytokine expression Regul Pept 143:104-108

Cramer CP, Thiels E, Alberts JR (1990) Weaning in rats: I. Maternal behavior Developmental psychobiology 23:479-493

Cripps A, Williams V (1975) The effect of pregnancy and lactation on food intake, gastrointestinal anatomy and the absorptive capacity of the small intestine in the albino rat British Journal of Nutrition 33:17-32

Darwin C (1859) On the Origin of Species by Means of Natural Selection, Or, The Preservation of Favoured Races in the Struggle for Life. J. Murray.

Date Y et al. (1999) Orexins, orexigenic hypothalamic peptides, interact with autonomic, neuroendocrine and neuroregulatory systems Proc Natl Acad Sci U S A 96:748-753

de Lecea L et al. (1998) The hypocretins: hypothalamus-specific peptides with neuroexcitatory activity Proc Natl Acad Sci U S A 95:322-327

Donovan B, Ten Bosch JVDW (1956) Precocious puberty in rats with hypothalamic lesions Nature 178:745

Edwards C, Abusnana S, Sunter D, Murphy K, Ghatei M, Bloom S (1999) The effect of the orexins on food intake: comparison with neuropeptide $Y$, melanin-concentrating hormone and galanin Journal of Endocrinology 160:R7-12

Egwuenu EJ, Fong AY, Pilowsky PM (2012) Intrathecal melanin-concentrating hormone reduces sympathetic tone and blocks cardiovascular reflexes American Journal of Physiology-Regulatory, Integrative and Comparative Physiology 303:R624-R632

Elias CF et al. (1998) Chemically defined projections linking the mediobasal hypothalamus and the lateral hypothalamic area J Comp Neurol 402:442-459

España RA, Berridge CW, Gammie SC (2004) Diurnal levels of Fos immunoreactivity are elevated within hypocretin neurons in lactating mice Peptides 25:1927-1934 
Ferssiwi A, Cardo B, Velley L (1987) Gustatory preference-aversion thresholds are increased by ibotenic acid lesion of the lateral hypothalamus in the rat Brain research 437:142-150

Furlong TM, Vianna DM, Liu L, Carrive P (2009) Hypocretin/orexin contributes to the expression of some but not all forms of stress and arousal European Journal of Neuroscience 30:1603-1614

Gao XB, van den Pol AN Melanin concentrating hormone: an inhibitory neuropeptide in the lateral hypothalamus. In: Soc Neurosci Abstr, 2000. vol 1. p 1151

Garcia MC, Lopez M, Gualillo O, Seoane LM, Dieguez C, Senaris RM (2003) Hypothalamic levels of NPY, MCH, and prepro-orexin mRNA during pregnancy and lactation in the rat: role of prolactin FASEB J 17:1392-1400

Geeraedts LM, Nieuwenhuys R, Veening JG (1990) Medial forebrain bundle of the rat: IV. Cytoarchitecture of the caudal (lateral hypothalamic) part of the medial forebrain bundle bed nucleus J Comp Neurol 294:537-568

Georgescu D et al. (2005) The hypothalamic neuropeptide melanin-concentrating hormone acts in the nucleus accumbens to modulate feeding behavior and forced-swim performance J Neurosci 25:2933-2940

González JA, Jensen LT, lordanidou P, Strom M, Fugger L, Burdakov D (2016) Inhibitory interplay between orexin neurons and eating Current Biology 26:2486-2491

Gonzalez MI, Vaziri S, Wilson CA (1996) Behavioral effects of alpha-MSH and MCH after central administration in the female rat Peptides 17:171-177

Gurdjian ES (1927) The diencephalon of the albino rat. Studies on the brain of the rat. no. 2 J Comp Neurol 43:1-114

Hagan JJ et al. (1999) Orexin A activates locus coeruleus cell firing and increases arousal in the rat Proc Natl Acad Sci U S A 96:10911-10916

Hara J et al. (2001) Genetic ablation of orexin neurons in mice results in narcolepsy, hypophagia, and obesity Neuron 30:345354

Harris GC, Aston-Jones G (2006) Arousal and reward: a dichotomy in orexin function Trends Neurosci 29:571-577

Haynes AC, Jackson B, Overend P, Buckingham RE, Wilson S, Tadayyon M, Arch JR (1999) Effects of single and chronic intracerebroventricular administration of the orexins on feeding in the rat Peptides 20:1099-1105

Kennedy AR et al. (2003) Effect of direct injection of melanin-concentrating hormone into the paraventricular nucleus: further evidence for a stimulatory role in the adrenal axis via SLC-1 J Neuroendocrinol 15:268-272

Kennedy AR, Todd JF, Stanley SA, Abbott CR, Small CJ, Ghatei MA, Bloom SR (2001) Melanin-concentrating hormone (MCH) suppresses thyroid stimulating hormone (TSH) release, in vivo and in vitro, via the hypothalamus and the pituitary Endocrinology 142:3265-3268

Knollema S, Brown ER, Vale W, Sawchenko PE (1992) Novel hypothalamic and preoptic sites of prepro-melanin-concentrating hormone messenger ribonucleic Acid and Peptide expression in lactating rats J Neuroendocrinol 4:709-717

Kokkotou E et al. (2009) Melanin-concentrating hormone (MCH) modulates C difficile toxin A-mediated enteritis in mice Gut 58:34-40

Lagos P, Torterolo P, Jantos H, Monti JM (2011) Immunoneutralization of melanin-concentrating hormone (MCH) in the dorsal raphe nucleus: effects on sleep and wakefulness Brain research 1369:112-118

Lin J-S, Sakai K, Vanni-Mercier G, Jouvet M (1989) A critical role of the posterior hypothalamus in the mechanisms of wakefulness determined by microinjection of muscimol in freely moving cats Brain research 479:225-240

Lin L et al. (1999) The sleep disorder canine narcolepsy is caused by a mutation in the hypocretin (orexin) receptor 2 gene Cell 98:365-376

Liu D, Diorio J, Day JC, Francis DD, Meaney MJ (2000) Maternal care, hippocampal synaptogenesis and cognitive development in rats Nature neuroscience 3:799

Ludwig DS et al. (2001) Melanin-concentrating hormone overexpression in transgenic mice leads to obesity and insulin resistance J Clin Invest 107:379-386

Mahler SV, Aston-Jones GS (2012) Fos activation of selective afferents to ventral tegmental area during cue-induced reinstatement of cocaine seeking in rats Journal of Neuroscience 32:13309-13325

Mahler SV, Moorman DE, Smith RJ, James MH, Aston-Jones G (2014) Motivational activation: a unifying hypothesis of orexin/hypocretin function Nature neuroscience 17:1298

Messina MM, Overton JM (2007) Cardiovascular effects of melanin-concentrating hormone Regulatory peptides 139:23-30

Miller CL, Hruby VJ, Matsunaga TO, Bickford PC (1993) Alpha-MSH and MCH are functional antagonists in a CNS auditory gating paradigm Peptides 14:431-440

Monzon ME, De Barioglio SR (1999) Response to novelty after i.c.v. injection of melanin-concentrating hormone (MCH) in rats Physiol Behav 67:813-817

Monzon ME, Varas MM, De Barioglio SR (2001) Anxiogenesis induced by nitric oxide synthase inhibition and anxiolytic effect of melanin-concentrating hormone (MCH) in rat brain Peptides 22:1043-1047.

Moret F, Guilland JC, Coudouel S, Rochette L, Vernier P (2004) Distribution of tyrosine hydroxylase, dopamine, and serotonin in the central nervous system of amphioxus (Branchiostoma lanceolatum): implications for the evolution of catecholamine systems in vertebrates Journal of Comparative Neurology 468:135-150 
Morgan HD, Watchus JA, Milgram NW, Fleming AS (1999) The long lasting effects of electrical simulation of the medial preoptic area and medial amygdala on maternal behavior in female rats Behavioural Brain Research 99:61-73

Morishige WK, Pepe GJ, Rothchild I (1973) Serum luteinizing hormone, prolactin and progesterone levels during pregnancy in the rat Endocrinology 92:1527-1530

Murray JF et al. (2000) Melanin-concentrating hormone, melanocortin receptors and regulation of luteinizing hormone release J Neuroendocrinol 12:217-223

Murray JF et al. (2006) Evidence for a Stimulatory Action of Melanin-Concentrating Hormone on Luteinising Hormone Release Involving MCH1 and Melanocortin-5 Receptors Journal of neuroendocrinology 18:157-167

Nahon JL, Presse F, Bittencourt JC, Sawchenko PE, Vale W (1989) The rat melanin-concentrating hormone messenger ribonucleic acid encodes multiple putative neuropeptides coexpressed in the dorsolateral hypothalamus Endocrinology 125:2056-2065

Naismith D, Richardson D, Pritchard AE (1982) The utilization of protein and energy during lactation in the rat, with particular regard to the use of fat accumulated in pregnancy British journal of nutrition 48:433-441

Nambu T, Sakurai T, Mizukami K, Hosoya Y, Yanagisawa M, Goto K (1999) Distribution of orexin neurons in the adult rat brain Brain research 827:243-260

Naufahu J, Cunliffe AD, Murray JF (2013) The roles of melanin-concentrating hormone in energy balance and reproductive function: Are they connected? Reproduction 146:R141-150

Nieuwenhuys R, Geeraedts LM, Veening JG (1982) The medial forebrain bundle of the rat. I. General introduction J Comp Neurol 206:49-81

Numan M (1974) Medial preoptic area and maternal behavior in the female rat Journal of comparative and physiological psychology 87:746

Numan M, Rosenblatt JS, Komisaruk BR (1977) Medial preoptic area and onset of maternal behavior in the rat Journal of comparative and physiological psychology 91:146

Numan M, Stolzenberg DS (2009) Medial preoptic area interactions with dopamine neural systems in the control of the onset and maintenance of maternal behavior in rats Front Neuroendocrinol 30:46-64

Pachoud B, Adamantidis A, Ravassard P, Luppi PH, Grisar T, Lakaye B, Salin PA (2010) Major impairments of glutamatergic transmission and long-term synaptic plasticity in the hippocampus of mice lacking the melanin-concentrating hormone receptor-1 I Neurophysiol 104:1417-1425

Parkes DG, Vale WW (1993) Contrasting actions of melanin-concentrating hormone and neuropeptide-E-I on posterior pituitary function Ann N Y Acad Sci 680:588-590

Peyron C, Tighe DK, van den Pol AN, de Lecea L, Heller HC, Sutcliffe JG, Kilduff TS (1998) Neurons containing hypocretin (orexin) project to multiple neuronal systems J Neurosci 18:9996-10015

Pfaff DW, Sakuma Y (1979) Deficit in the lordosis reflex of female rats caused by lesions in the ventromedial nucleus of the hypothalamus The Journal of physiology 288:203-210

Qu D et al. (1996) A role for melanin-concentrating hormone in the central regulation of feeding behaviour Nature 380:243247

Rondini TA, Donato J, Jr., Rodrigues Bde C, Bittencourt JC, Elias CF (2010) Chemical identity and connections of medial preoptic area neurons expressing melanin-concentrating hormone during lactation J Chem Neuroanat 39:51-62

Sakurai T et al. (1998) Orexins and orexin receptors: a family of hypothalamic neuropeptides and $G$ protein-coupled receptors that regulate feeding behavior Cell 92:573-585

Satinoff E, Shan SY (1971) Loss of behavioral thermoregulation after lateral hypothalamic lesions in rats Journal of Comparative and Physiological Psychology 77:302

Seeber RM, Smith JT, Waddell BJ (2002) Plasma leptin-binding activity and hypothalamic leptin receptor expression during pregnancy and lactation in the rat Biology of reproduction 66:1762-1767

Segal-Lieberman G et al. (2003) Melanin-concentrating hormone is a critical mediator of the leptin-deficient phenotype Proc Natl Acad Sci U S A 100:10085-10090

Shimada M, Tritos NA, Lowell BB, Flier JS, Maratos-Flier E (1998) Mice lacking melanin-concentrating hormone are hypophagic and lean Nature 396:670-674

Shirley B (1984) The food intake of rats during pregnancy and lactation Laboratory animal science 34:169-172

Smart J, Dobbing J (1971) Vulnerability of developing brain. II. Effects of early nutritional deprivation on reflex ontogeny and development of behaviour in the rat Brain Research 28:85-95

Smith DG et al. (2006) Melanin-concentrating hormone-1 receptor modulates neuroendocrine, behavioral, and corticolimbic neurochemical stress responses in mice Neuropsychopharmacology 31:1135-1145

Stephan FK, Zucker I (1972) Circadian rhythms in drinking behavior and locomotor activity of rats are eliminated by hypothalamic lesions Proceedings of the National Academy of Sciences 69:1583-1586

Sun G, Narita K, Murata T, Honda K, Higuchi T (2003) Orexin-A Immunoreactivity and Prepro-Orexin mRNA Expression in Hyperphagic Rats Induced By Hypothalamic Lesions and Lactation Journal of neuroendocrinology 15:51-60 
Sunn N et al. (2002) Circulating relaxin acts on subfornical organ neurons to stimulate water drinking in the rat Proceedings of the National Academy of Sciences 99:1701-1706

Theodosis DT (2002) Oxytocin-secreting neurons: a physiological model of morphological neuronal and glial plasticity in the adult hypothalamus Frontiers in neuroendocrinology 23:101-135

Thiels E, Alberts JR, Cramer CP (1990) Weaning in rats: Il. Pup behavior patterns Developmental psychobiology 23:495-510

Trujillo M, Spuch C, Carro E, Senaris R (2011) Hyperphagia and central mechanisms for leptin resistance during pregnancy Endocrinology 152:1355-1365

Tsujino N, Sakurai T (2009) Orexin/hypocretin: a neuropeptide at the interface of sleep, energy homeostasis, and reward system Pharmacological reviews 61:162-176

Tsunematsu T et al. (2014) Optogenetic manipulation of activity and temporally controlled cell-specific ablation reveal a role for MCH neurons in sleep/wake regulation Journal of Neuroscience 34:6896-6909

Vaughan JM, Fischer WH, Hoeger C, Rivier J, Vale W (1989) Characterization of melanin-concentrating hormone from rat hypothalamus Endocrinology 125:1660-1665

Veening JG, Swanson LW, Cowan WM, Nieuwenhuys R, Geeraedts LM (1982) The medial forebrain bundle of the rat. II. An autoradiographic study of the topography of the major descending and ascending components J Comp Neurol 206:82-108

Verret $L$ et al. (2003) A role of melanin-concentrating hormone producing neurons in the central regulation of paradoxical sleep BMC Neurosci 4:19

Verty AN, Allen AM, Oldfield BJ (2010) The endogenous actions of hypothalamic peptides on brown adipose tissue thermogenesis in the rat Endocrinology 151:4236-4246

Volkoff H, Bjorklund JM, Peter RE (1999) Stimulation of feeding behavior and food consumption in the goldfish, Carassius auratus, by orexin-A and orexin-B Brain research 846:204-209

Wang J, Osaka T, Inoue S (2001) Energy expenditure by intracerebroventricular administration of orexin to anesthetized rats Neuroscience letters 315:49-52

Wang J-B, Murata T, Narita K, Honda K, Higuchi T (2003) Variation in the expression of orexin and orexin receptors in the rat hypothalamus during the estrous cycle, pregnancy, parturition, and lactation Endocrine 22:127-134

Yoshimatsu H, Niijima A, Oomura Y, Yamabe K, Katafuchi T (1984) Effects of hypothalamic lesion on pancreatic autonomic nerve activity in the rat Brain research 303:147-152 



\title{
Chapter 2
}

\section{Neuroanatomical Structure of the MCH System}

\author{
Bittencourt $J \mathrm{C}^{1}$ and Diniz $\mathrm{GB}^{1}$ \\ 'These authors contributed equally to this work
}

As published in: Pandi-Perumal S., Torterolo P., Monti J. (eds) MelaninConcentrating Hormone and Sleep. Springer, Cham. 2018. DOI: 10.1007/978-3-319-75765-0_1 



\title{
Neuroanatomical Structure of the MCH System
}

\author{
Jackson C. Bittencourt and Giovanne B. Diniz
}

\begin{abstract}
The mammalian melanin-concentrating hormone $(\mathrm{MCH})$ system has been studied for almost 30 years as of the writing of this chapter. The understanding of any neural system starts with its structure, as the anatomical organization of its peptides and receptors provides important clues about its physiological activity. With that in mind, the MCH system will be reviewed as a whole in the first segment of this chapter, including the genes and proteins that comprise this system. In the following segment, a description of the morphological and neurochemical characteristics of MCH neurons will be provided, including the areas in the central nervous system where these neurons are found and their projection pathways and targets. In the last segment, the receptors for $\mathrm{MCH}$ will be briefly discussed, and the available anatomical information about these receptors will be presented to the reader. By the end of this chapter, the reader should be able to identify the sites of MCH synthesis, the major targets of $\mathrm{MCH}$ action, and the areas responsive to $\mathrm{MCH}$ owing to the presence of its receptor.
\end{abstract}

\section{List of Abbreviations}

3V Third ventricle

3Vdc Third ventricle dorsal cap

aa Amino acid(s)

AHA Anterior hypothalamic area

\footnotetext{
J. C. Bittencourt $(\bowtie)$

Laboratory of Chemical Neuroanatomy, Department of Anatomy, Institute of Biomedical Sciences, University of Sao Paulo, Sao Paulo, Brazil

Centre for Neuroscience and Behavior, Institute of Psychology, University of Sao Paulo, Sao

Paulo, Brazil

e-mail: jcbitten@icb.usp.br

G. B. Diniz

Laboratory of Chemical Neuroanatomy, Department of Anatomy, Institute of Biomedical

Sciences, University of Sao Paulo, Sao Paulo, Brazil

e-mail: giovanne.diniz@usp.br
} 


\begin{tabular}{|c|c|}
\hline ANP & Atrial natriuretic peptide \\
\hline$A R O M$ & Antisense-RNA-overlapping-MCH gene \\
\hline AVP & Arginine vasopressin \\
\hline BNST & Bed nucleus of the stria terminalis \\
\hline CART & Cocaine- and amphetamine-regulated transcript \\
\hline CNS & Central nervous system \\
\hline $\mathrm{CRF}$ & Corticotropin-releasing factor \\
\hline f & Fornix \\
\hline GAD67 & Glutamate decarboxylase $67 \mathrm{kDa}$ \\
\hline GPCR & G protein-coupled receptor \\
\hline ic & Internal capsule \\
\hline IHy & Incertohypothalamic area \\
\hline IRE & Interferon-gamma responsive element \\
\hline LDT & Laterodorsal tegmental nucleus \\
\hline LHA & Lateral hypothalamic area \\
\hline $\mathrm{MCH}$ & Melanin-concentrating hormone (peptide) \\
\hline MCHR 1 & Melanin-concentrating hormone receptor 1 (peptide) \\
\hline MCHRI & Melanin-concentrating hormone receptor 1 (primate gene) \\
\hline Mchrl & Melanin-concentrating hormone receptor 1 (rodent gene) \\
\hline MCHR2 & Melanin-concentrating hormone receptor 2 (peptide) \\
\hline MCHR2 & Melanin-concentrating hormone receptor 2 (primate gene) \\
\hline $\mathrm{ME}$ & Median eminence \\
\hline$m f b$ & Medial forebrain bundle \\
\hline $\mathrm{mt}$ & Mammillothalamic tract \\
\hline MGOP & MCH-gene-overprinted-polypeptide \\
\hline MPOA & Medial preoptic area \\
\hline NEI & Neuropeptide glutamic acid-isoleucine \\
\hline NGE & Neuropeptide glycine-glutamic acid \\
\hline NPY & Neuropeptide Y \\
\hline opt & Optic tract \\
\hline ORF & Open reading frame \\
\hline ORX & Orexin/hypocretin \\
\hline PACAP & Pituitary adenylate cyclase-activating peptide \\
\hline $\mathrm{PC}$ & Prohormone convertase(s) \\
\hline PH & Posterior hypothalamic area \\
\hline Pmch & Pro-melanin-concentrating hormone (rodent gene) \\
\hline PMCH & Pro-melanin-concentrating hormone (primate gene) \\
\hline PMCHLI & Pro-melanin-concentrating hormone linked gene 1 (chimeric gene) \\
\hline PMCHL2 & Pro-melanin-concentrating hormone linked gene 2 (chimeric gene) \\
\hline pmPRt & Paramedian pontine reticular formation \\
\hline ppMCH & Prepro-melanin-concentrating hormone \\
\hline $\mathrm{PVH}$ & Paraventricular nucleus of the hypothalamus \\
\hline REM & Rapid eye movement sleep \\
\hline SDL & Subcoeruleus/sublaterodorsal nucleus \\
\hline SLC-1 & Somatostatin-like coupled receptor 1 , same as MCHR 1 \\
\hline
\end{tabular}




$\begin{array}{ll}\text { SP } & \text { Substance P } \\ \text { SSTR } & \text { Somatostatin receptor(s) } \\ \text { TM } & \text { Transmembrane } \\ \text { TMdm } & \text { Tuberomammillary nucleus, dorsomedial part } \\ \text { Tu } & \text { Olfactory tubercle } \\ \text { X-ir } & \text { Immunoreactive to X } \\ \text { ZI } & \text { Zona incerta } \\ \alpha \text {-MSH } & \alpha \text {-Melanocyte-stimulating hormone }\end{array}$

\section{Introduction}

The melanin-concentrating hormone $(\mathrm{MCH})$ system is a complex vertebrate neuropeptidergic system, composed of several neuropeptides and multiple receptor subtypes that span several clades. Although $\mathrm{MCH}$ was initially isolated from the teleost pituitary as a chromophore modulator (Kawauchi et al. 1983), less than a decade was necessary for the description of mammalian orthologues of its gene (Pmch/PMCH) (Nahon et al. 1989, 1993; Presse et al. 1990). Simultaneously, the amino acid (aa) sequence of the peptides encoded by these genes (Vaughan et al. 1989) was described, and their distribution in the mammalian brain soon followed (Bittencourt et al. 1992). The description of the neuroanatomical structure of the MCH system was then followed by a profusion of functional works, which slowly started to unveil the numerous roles played by its peptides in mammalian physiology. Among these functions, and of particular interest to this work, are the modulation of motivated behaviors (reviewed in Diniz and Bittencourt 2017) and of the sleep-wake cycle (reviewed in Ferreira et al. 2017a).

It is necessary, however, to clarify the definitions of "motivated behaviors" and "sleep-wake cycle" employed in this chapter, as these concepts are often used in the literature without further consideration. The most common conception of motivated behaviors is as behaviors that the animal performs to meet homeostatic needs. This description is true of ingestive behavior, perhaps the most thoroughly studied motivated behavior. However, this definition fails to include, for example, mating and maternal behaviors; both of these types of behavior may even result in a worsening of the animal's metabolic needs, but it performs these actions nevertheless because they are necessary for the maintenance of the species. Motivated behaviors could therefore be redefined as actions that the animal executes that are necessary to its survival or the survival of its species. Although this definition can be applied to all behaviors discussed so far, it opens up the question of whether sleeping can be considered a motivated behavior. For the purposes of this work, it will not be. While other behaviors require the animal to interact with exogenous elements (e.g., food, mate, pups) for their full completion, sleep can occur regardless of exogenous elements. Therefore, we will define motivated behaviors in this chapter as actions that the animal executes toward exogenous agents that are necessary for its survival or the 
survival of its species. Sleep, on the other hand, will be defined as "a periodic, complex intrinsic state alteration that encompasses synaptic alterations, hormone secretion, and consciousness suspension and that is necessary for the support of several other physiological processes."

As a complex physiological process, the sleep-wake cycle is sustained in the central nervous system (CNS) by an equally complex network of nuclei, connections, neurotransmitters, and neuromodulators. Central in this network is the hypothalamus and, in particular, the lateral hypothalamic area (LHA), which has been long known to be an integrative center of homeostatic controls and motivated behaviors, receiving inputs from and sending outputs to the whole CNS (Diniz and Bittencourt 2017). Although the importance of the LHA for several behaviors has been recognized for a long time, most of its inner workings have remained a black box for decades, mainly owing to its remarkable cyto- and chemoarchitecture and the complexity of its connectivity (Simerly 2015). The study of the LHA was further complicated by the extensive presence of the medial forebrain bundle $(m f b)$ in this area, as the LHA has been considered the bed nucleus of the $m f b$ (Nieuwenhuys et al. 1982). It was only after the discovery of LHA-specific neurochemical markers (e.g., MCH, orexin/ hypocretin [ORX] and cocaine- and amphetamine-regulated transcript [CART]) that this area could be explored in a more detailed way (Bittencourt et al. 1992; Sakurai et al. 1998; de Lecea et al. 1998; Douglass et al. 1995). The discovery of these markers, in addition to the development of new methodologies, such as optogenetics, chemogenetics, in vivo electrophysiology, polysynaptic viral tracing, calcium imaging, and miniaturized fluorescence microscopes, forever changed the way we understand the LHA and other hubs related to the control of the sleep-wake cycle.

The first attempts to anatomically describe the modulatory centers of sleep and wakefulness, however, predate these techniques by far and can be traced to the work of von Economo, in 1930. After attending to patients during the encephalitis epidemic of 1917-1926, von Economo noticed that some of his patients displayed a subset of signs and symptoms related to sleep, which he called encephalitis lethargica. Interestingly, while some patients displayed increased sleep time, others had a decrease, even though they were all part of the same viral epidemic. The correlation between symptoms and the location of the lesions in these patients led von Economo to propose a dual model of sleep control: the brain stem as the wakefulness-promoting center and the anterior hypothalamus as the sleep-promoting center (Von Economo 1930). Although simple, von Economo's model was surprisingly accurate and paved the way for many other nuclei to be included in this network in the following decades (Saper 2013). Likewise, several neuromodulators, including $\mathrm{MCH}$ and other lateral hypothalamic neuropeptides, were also implicated in this control system as new evidence was incorporated into the model. In the next part of this chapter, the basic aspects of the $\mathrm{MCH}$ system and its anatomical structure in the mammalian CNS will be reviewed. 


\section{The MCH System}

In broad strokes, the mammalian MCH system can be split into two components: a peptidergic element and a receptor element. The peptidergic component is composed of a single gene, $\mathrm{Pmch} / \mathrm{PMCH}$, which encodes a precursor that originates three mature peptides: $\mathrm{MCH}$, neuropeptide glycine-glutamic acid (NGE), and neuropeptide glutamic acid-isoleucine (NEI). While substantial anatomical and functional data have been obtained for MCH and NEI (as reviewed in Diniz and Bittencourt 2017; Bittencourt and Celis 2008), the status of NGE as a neuroactive substance has remained controversial. It has been suggested that NGE may modulate cAMP production (Hintermann et al. 2001), but further studies are needed to give us a better understanding of this putative peptide. The receptor component consists of two genes, Mchrl/MCHRl and MCHR2, each encoding one receptor subtype (MCHR1 and MCHR2, respectively). The subtype 2 receptor is not ubiquitous among vertebrates, however, as a functional MCHR2 cannot be found in species of the Glires superorder. No receptor is known for NEI or NGE. The discovery of their receptors or their mechanisms of action, if existent, could represent the next breakthrough in the understanding of the $\mathrm{MCH}$ system. In this section, the general characteristics of each component of the MCH system will be explored.

\subsection{MCH, NEI, and NGE}

Since there is substantial complexity in the $\mathrm{MCH}$ system across different vertebrate clades, which goes beyond the scope of this work, the focus of this section will be on mammalian species, more specifically on data from rats, mice, and humans. As the Pmch/PMCH gene is remarkably conserved among mammals, it is likely that observations made on the cited animals can be extrapolated to other species.

The evidence collected so far point to the existence of a single authentic Pmch gene in mammals, spanning approximately $1.4 \mathrm{~kb}$. In rats ( $r P m c h$-gene ID, 24659), this gene is located on chromosome $7 \mathrm{q} 13$ and overlaps with the PARP1 binding protein-coding gene (Parpbp) (Nahon et al. 1992). In the same locus are the genes coding for insulin-like growth factor 1 (Igfl), nucleoporin 37 (Nup37), and WASH complex subunit 3 (Washc3). The mouse gene (mPmch-gene ID, 110312) is located on chromosome $10 \mathrm{C} 11043.7 \mathrm{cM}$, and, as in the rat, it overlaps with the Parpbp gene and is flanked by Igfl, Nup37, and Washc3 genes. Finally, the human gene $(P M C H$ - gene ID, 5367) is located on chromosome 12q23.2 and has a similar synteny to the murine species (Pedeutour et al. 1994).

In all these species, the Pmch/PMCH gene follows the same basic structure, i.e., it is formed by three exons (Exon 1 coding sequence, 249 base pairs [bp]; Exon 2, 199 bp; Exon 3 coding sequence, $47 \mathrm{bp}$ ), in addition to two introns and untranslated $5^{\prime}$ and $3^{\prime}$ sequences whose lengths differ between species (Nahon et al. 1989; Breton et al. 1993a; Thompson and Watson 1990; Presse et al. 1990). Figure 1 illustrates the 


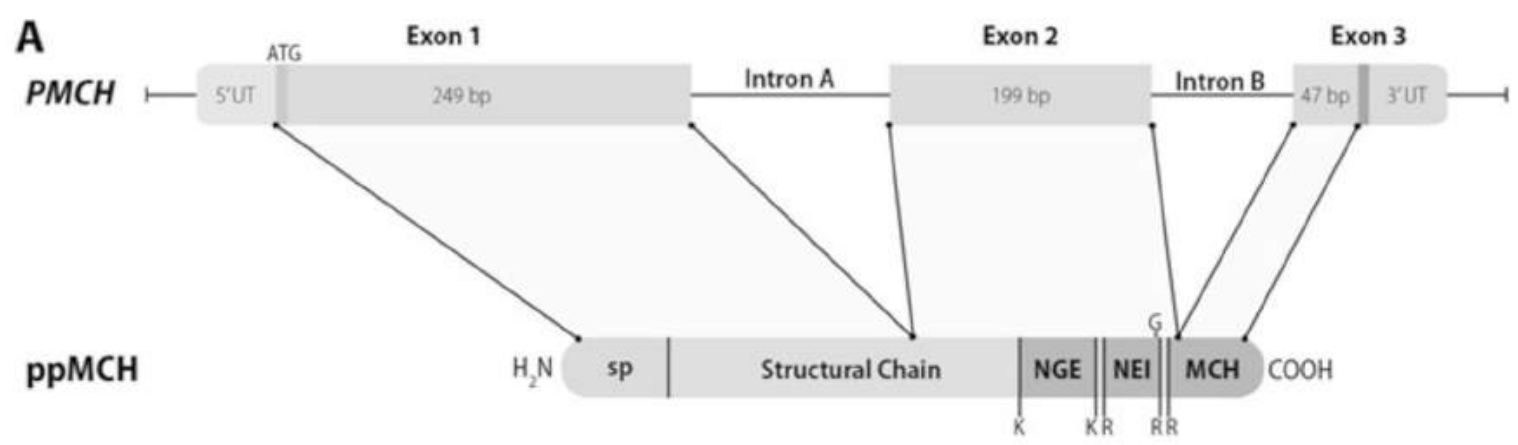

B

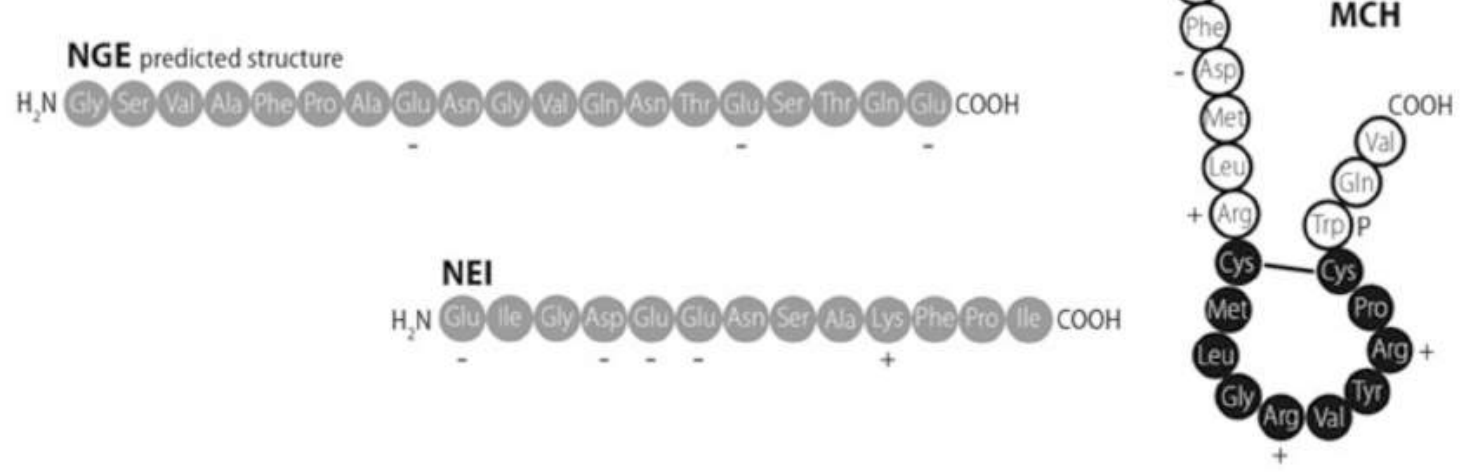

Fig. 1 Structure of the $P M C H$ gene and its family of peptides. Schematic representation of the human $P M C H$ gene and the MCH, NEI, and NGE peptides. (a) The PMCH gene is composed of three exons interrupted by two intronic sequences. The first exon corresponds to the signal peptide (sp) and the first part of the structural chain of prepro-MCH (ppMCH). The second exon codes for the remaining segment of the structural chain, NGE, NEI, and the first three amino acids of MCH. The third exon will complete the fourth codon corresponding to $\mathrm{MCH}$ and the final 15 amino acids. The sites of cleavage by prohormone convertases are indicated in the ppMCH structure by " $\mathrm{K}$ " and "R." The amidation site of NEI is indicated by "G." (b) The residue sequences for MCH, NEI, and the predicted structure of NGE. Plus and minus signs indicate the polarity of electrically charged amino acids. The letter "P" indicates a residue that is important for binding potentiation of $\mathrm{MCH}$ $\left(\operatorname{Trp}{ }^{17}\right)$. White circles denote residues that are not essential for $\mathrm{MCH}$ function, while black circles denote the necessary amino acids for $\mathrm{MCH}$ binding. Gray circles are used for peptides without known binding properties

$P M C H$ gene structure. In the upstream flanking region of the Pmch/PMCH gene, several promoter sequences are found, including two AP-1-binding sequences, one interferon- $\gamma$ response element (IRE), and partial sequences of a glucocorticoid response element (Viale et al. 1997). Through AP-1 sites, the transcription of Pmch may respond to the FOS/JUN transcription factors, suggesting that FOS may be an adequate marker for the activity of $\mathrm{MCH}$-containing neurons. Through the IRE, MCH neurons may respond to immunological events, such as sickness behavior or hypothalamic inflammation. The glucocorticoid response element may act to couple $\mathrm{MCH}$ populations to the defensive stress response/arousal circuits. Between the two AP- 1 sequences, there is a CAAT box, and approximately $27 \mathrm{bp}$ upstream of the cap nucleotide is a TATA box. These two sequences are good indicators that the area is the regulatory zone of $P M C H$ transcription initiation (Viale et al. 1997). 
As mentioned above, Exon 1 contains a 249 bp coding sequence, including the ATG start codon, in addition to the $5^{\prime}$ untranslated region, whose length depends on the species. The $5^{\prime}$ untranslated sequence is highly conserved between humans and mice and presents a palindromic sequence juxtaposed to the start codon, which could act as part of a transcription control mechanism through the formation of a hairpin. The substitutions in the rat sequence do not support the presence of a folding structure in this species (Breton et al. 1993a). There is a second putative ATG start codon four positions downstream of the first codon. Although both are viable starting points, several works support the first ATG codon as the primary initiation locus (Nahon et al. 1989; Breton et al. 1993a; Presse et al. 1990). There is high identity among all three species concerning the coding region of Exon 1, with the mouse equidistant from humans and rats and a slightly bigger difference between rats and humans ( $\mathrm{R}$ vs. $\mathrm{M}, 86.75 \%$; $\mathrm{R}$ vs. $\mathrm{H}, 82.33 \%$; $\mathrm{H}$ vs. $\mathrm{M}, 86.37 \%$ ). It is noteworthy that Exon 1 is the least conserved of the three exons.

Exons 1 and 2 are separated by Intron $\mathrm{A}$, a sequence whose length varies depending on the species, ranging from 302 to 350 bp. Exon 2 is 199 bp in length and is slightly more conserved among the three mammalian species, contrasting with the upstream exon by having higher similarity between the murine species than between the murine and human sequences (R vs. M, 94.48\%; R vs. H, 87.94\%; H vs. M, $89.95 \%)$. After an intermission consisting of Intron B, with lengths ranging from 250 to $271 \mathrm{bp}$, Exon 3 completes the coding portion of the gene with $47 \mathrm{bp}$. The Exon 3 coding region is remarkably conserved among mammalian species ( $R$ vs. $M$, 96\%; R vs. H, 94\%; H vs. M, 96\%). Interestingly, the intronic regions display a higher degree of identity between rats and mice (86-88\%) than the exonic sequences do, but a much lower degree between mice and humans (60-70\%) (Thompson and Watson 1990; Breton et al. 1993a, b). Following the stop codon, there is a $3^{\prime}$ untranslated sequence of variable length that shows a high content of AT motifs and a putative polyadenylation site (ATTAAA), possibly responsible for posttranslational regulation of the $\mathrm{Pmch} / \mathrm{PMCH} \mathrm{cDNA}$, as well as two opposite repeat sequences that could contribute to the formation of a hairpin structure (Breton et al. 1993a).

The Pmch/PMCH open reading frame (ORF) is a highly conserved sequence of 495 bp that encodes a prepro-MCH (ppMCH) of 165 aa in all three species (Fig. 1). The first 63 nucleotides (Exon 1) encode a signal peptide that is cleaved by signal peptidases at $\mathrm{Gly}^{21}$, generating a pro-MCH of 144 aa. The remaining 186 nucleotides of Exon 1 and the first 75 of Exon 2 combine after Intron $\mathrm{A}$ is spliced to form the 87-aa-long structural chain. Also in Exon 2 is the 124 nucleotides that code for NGE (19 aa), NEI (13 aa), and the first three amino acids of $\mathrm{MCH}$. Intron B is inserted in the codon that originates $\mathrm{Met}^{4}$ of $\mathrm{MCH}$. After this intermission, the 47 nucleotides of the Exon 3 coding sequence complete $\mathrm{Met}^{4}$ and encode the final 15 residues of MCH (19 aa in total) (Vaughan et al. 1989; Nahon et al. 1989; Breton et al. 1993a; Thompson and Watson 1990). After the formation of pro-MCH, the activity of prohormone convertases (PCs) will be essential for the formation of mature NGE, NEI, and MCH. Neuropeptide G-E is separated from the structural chain by cleavage of ppMCH Lys ${ }^{109}$ and separated from NEI through the cleavage of the dibasic ppMCH residues Lys ${ }^{129}-\operatorname{Arg}^{130}$. Neuropeptide E-I is separated 
from $\mathrm{MCH}$ through cleavage of the dibasic ppMCH residues $\mathrm{Arg}^{145}-\mathrm{Arg}^{146}$. Although not completely elucidated, the cleavage of ppMCH appears to be dependent on several PCs. While PC7, PACE4, PC1/3, PC2, PC5/6-A, and PC5/6-B seem to be able to cleave the $\mathrm{Arg}^{145}-\mathrm{Arg}^{146}$ residues to generate mature $\mathrm{MCH}, \mathrm{PC} 2$ appears to be exclusively responsible for the cleavage of residues Lys ${ }^{129}-\operatorname{Arg}^{130}$ to generate NEI-G, which will be further processed to generate mature NEI (Viale et al. 1999). The importance of PC2 in the maturation of the $\mathrm{MCH}$ peptide family is further supported by ample colocalization between Pmch mRNA and PC2 immunoreactivity or Pcsk2 expression in the hypothalamus. On the other hand, only $15-20 \%$ of hypothalamic Pmch mRNA-expressing cells are immunoreactive to PC1/3 (Viale et al. 1999). Further studies are necessary to fully clarify the action of PCs on the formation of mature $\mathrm{MCH}$ and NEI. While some information is available regarding $\mathrm{MCH}$ and $\mathrm{NEI}$, virtually nothing is known about NGE synthesis, as no one has provided evidence for its synthesis in vivo or in vitro. One possibility is that, instead of NGE, pro-MCH encodes an neuropeptide PE, spanning residues Pro ${ }^{104}$ to $\mathrm{Glu}^{128}$, as another Lys residue is found in position 103 and could act as a cleavage site, although little support for this theory is found in the literature (Nahon et al. 1989; Breton et al. 1993a).

While NGE has no putative post-translational modification and is predicted to be linear, NEI undergoes C-terminal amidation on ppMCH residue Gly ${ }^{144}$ to reach its mature form but stays linear (Parkes and Vale 1992). On the other hand, MCH has a cyclic structure due to a disulfide bond between residues $\mathrm{Cys}^{7}$ and $\mathrm{Cys}^{16}$ (Nahon et al. 1989; Vaughan et al. 1989) (Fig. 1). This cyclic portion appears to be fundamental for $\mathrm{MCH}$ activity, as disturbances of the ring structure strongly decrease its activity (Macdonald et al. 2000). Furthermore, the cyclic region contains two positive residues, $\operatorname{Arg}^{11}$ and $\operatorname{Arg}^{14}$, which confer a predominantly positive charge on the ring. While the substitution of $\operatorname{Arg}^{14}$ has minimal effects on the binding properties of $\mathrm{MCH}$, the loss of $\mathrm{Arg}^{11}$ virtually abolishes its binding to the $\mathrm{MCH}$ receptor. While the cyclic structure is necessary for the activity of $\mathrm{MCH}, \operatorname{Trp}^{17}$, located outside the ring, appears to potentiate $\mathrm{MCH}$ activity. Despite the binding activity being focused on the ring structure, the whole $\mathrm{MCH}$ amino acid sequence is conserved in mammals. The same is observed in NEI, whose aa sequence has $100 \%$ identity among humans, mice, and rats. Neuropeptide G-E, on the other hand, has a single substitution differing between humans and mice and four substitutions differing between rats and mice, suggesting less conservation pressure on this peptide than on the other peptides in the MCH family (Breton et al. 1993a).

In addition to the "canonical" MCH, NEI, and NGE, there are several other transcription products that may arise from the Pmch/PMCH gene. MCH-gene-overprintedpolypeptide (MGOP) is a putative peptide that arises from alternative splicing of the $P M C H$ gene. Both in rats and in mice, MGOP originates from the transcription of Exon 1, total absence of Exon 2, and a frameshifted transcription of Exon 3, which generates a protein whose $\mathrm{N}$-terminal region is conserved when compared with ppMCH but whose C-terminal region is entirely different (Toumaniantz et al. 1996, 2000). Immunoreactivity to MGOP is found in MCH-immunoreactive ( $\mathrm{MCH}$-ir) neurons of rodents, but its function is unknown at the time (Allaeys et al. 2004). The antisense-RNA-overlapping$\mathrm{MCH}(A R O M)$ gene is found in the opposite DNA strand corresponding to the locus of 
$P M C H$. This gene has homologues in rats, mice, and humans and spans approximately $34 \mathrm{~kb}$ of genomic DNA. It has a complex genomic structure, containing over 12 exons and multiple initiation sites, possibly generating several coding and noncoding transcripts. Some of the proteins that possibly originate from AROM display nucleotide strand-binding properties, suggesting that these products may regulate gene transcription (Borsu et al. 2000; Moldovan et al. 2012). Finally, there are two PMCH-linked chimeric genes in primates, termed PMCHL1 and PMCHL2, which have partial homology to the authentic PMCH gene and are found on chromosomes 5p14 and 5q13, respectively. Preliminary data suggests that the products of these genes are long noncoding RNAs, with a trans-spliced transcript of PMCHLl being expressed in the CNS and putatively modulating PMCH mRNA expression (Breton et al. 1993b; Courseaux and Nahon 2001; Presse et al. 2014).

\subsection{Melanin-Concentrating Hormone Receptor 1}

MCHR 1 is found in virtually all known vertebrates. It was the first receptor discovered that has a selective binding affinity for $\mathrm{MCH}$, although some years passed between its description and the discovery of its natural ligand. The hunt for an $\mathrm{MCH}$ receptor started just 4 years after the description of the mammalian protein, with the development of a tritiated $\mathrm{MCH}$ analog that could be used as a radioligand to locate binding sites for MCH (Drozdz et al. 1993). However, a tenfold decrease in the activity of this analog compared with $\mathrm{MCH}$ prompted those authors to develop $\mathrm{N}$ - and C-terminal-iodinated $\mathrm{MCH}$ analogs that retained their full biological activity. Using these iodinated analogs, they were able to detect $\mathrm{MCH}$ binding in several cell lines, including G4F-7, B16F-1, G4F, human RE melanoma, COS-7, and PC12. No binding was detected for $\mathrm{CHO}$ cells or human fibroblasts. Competition experiments revealed that $\alpha$-melanocyte-stimulating hormone ( $\alpha-\mathrm{MSH})$, neuropeptide Y (NPY), pituitary adenylate cyclase-activating peptide (PACAP), and substance P (SP) were all unable to displace the iodinated $\mathrm{MCH}$ analogs, with the exception of rat atrial natriuretic peptide (ANP), which had two- to fourfold lower affinity than $\mathrm{MCH}$ (Drozdz and Eberle 1995; Drozdz et al. 1995). Further works expanded the number of MCH-binding cell lines, including SVK14, A-431, and SCC-25 (Burgaud et al. 1997) and reinforced the specificity of $\mathrm{MCH}$ binding, as corticotropin-releasing factor (CRF), arginine vasopressin (AVP), NEI, and MGOP were also not able to displace the radioligand.

A significant advance in the identification of the $\mathrm{MCH}$ receptor was made by Kolakowski et al. (1996). These authors identified a genomic DNA fragment that matched the orphan G protein-coupled receptor (GPCR) GPR24, which had significant homology to somatostatin receptors (SSTRs) and could be mapped to the human chromosome 22, locus q13.3. Although this receptor shared approximately $40 \%$ of its residues with SSTRs in the predicted transmembrane (TM) domains and had several key residues in critical positions for the binding activity of somatostatin, there was no evidence of binding to somatostatin-14 or opioid ligands. For this 
reason, this receptor was renamed somatostatin-like coupled receptor 1 (SLC-1). Further refinements of the knowledge regarding SLC-1 were provided by Lakaye et al. (1998), who identified the rat orthologue of SLC-1, described its gene sequence, including a previously unidentified intron, and located the consensus sites for glycosylation and phosphorylation. The ligand of SLC-1, however, remained unknown.

In 1999, a major leap in the understanding of the $\mathrm{MCH}$ peptidergic system occurred when five groups identified MCH as the ligand of SLC-1 (Lembo et al. 1999; Bachner et al. 1999; Chambers et al. 1999; Saito et al. 1999; Shimomura et al. 1999). These groups employed the method of "reverse pharmacology," transfecting cell lines with SLC-1, exposing those cells to either brain extracts or panels of neuroactive substances and then measuring parameters of receptor activation such as intracellular $\mathrm{Ca}^{2+}$ mobilization, cAMP production, or $\mathrm{K}^{+}$currents. In all cases, $\mathrm{MCH}$ was the only neuroactive substance capable of eliciting a response in the transfected cell lines, with an $\mathrm{EC}_{50}$ that ranged from $0.2 \mathrm{nM}$ to approximately $120 \mathrm{nM}$ depending on the cell line, stability of transfection, and response measured. These works also demonstrated that other neuroactive substances have no competitive activity and started to dissect the intracellular pathways of MCHR 1 activation.

All those works contributed to a clear picture of $\mathrm{MCH}$ receptor 1 . In the rat, this receptor is encoded by the Mchrl gene (Gene ID: 83567), which is located on chromosome 7q34. The coding sequences are composed of two exons of $82 \mathrm{bp}$ and $977 \mathrm{bp}$, separated by an intron of $1265 \mathrm{bp}$. The human MCHRI gene (Gene ID: 2847), on the other hand, is found on chromosome 22q13.2 and has an intronic sequence of $1214 \mathrm{bp}$ (Fig. 2). The intron position is the same in humans and rodents, splitting the codon of Gly ${ }^{28}$ into a G on Exon 1 and a GG (rat) or GA (human) in Exon 2. The resulting protein is 353 aa long in both species, forming the characteristic seven-TM domain structure of GPCRs. The aa sequence of this protein predicts three consensus sites for asparagine-linked glycosylation in the extracellular $\mathrm{N}$-terminus, two potential phosphorylation sites for protein kinase $\mathrm{A}$ in the second and third intracellular loops, six for protein kinase $\mathrm{C}$ in the second and third intracellular loops and in the C-terminal portion, and one for protein kinase CK2 in the C-terminal portion. The human and rat proteins share $96 \%$ identity, indicating high conservation pressure on this receptor. The three-dimensional model of MCHR 1 predicts a hydrophilic pocket between TM domains 3 and 7 and another among TM domains 4,5 , and 6 , which are separated by a central hydrophobic region (Macdonald et al. 2000) (Fig. 2).

\subsection{The Melanin-Concentrating Hormone Receptor 2}

Two years after the discovery of MCHR1, six groups were able to identify another receptor for MCH (An et al. 2001; Hill et al. 2001; Mori et al. 2001; Rodriguez et al. 2001; Sailer et al. 2001; Wang et al. 2001). Using the consensus structure of GPCRs and the available sequence of $M C H R l$, these groups were able to search human 
A

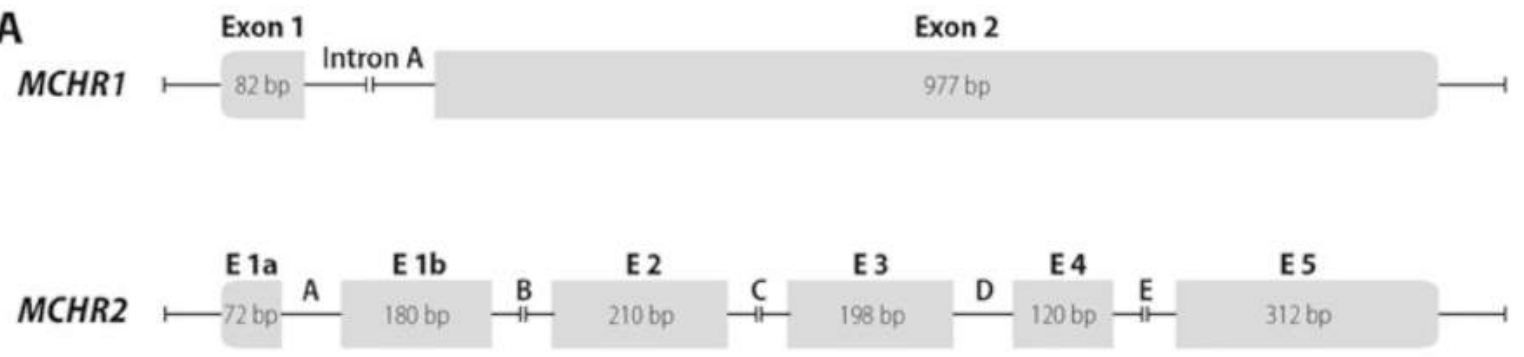

B
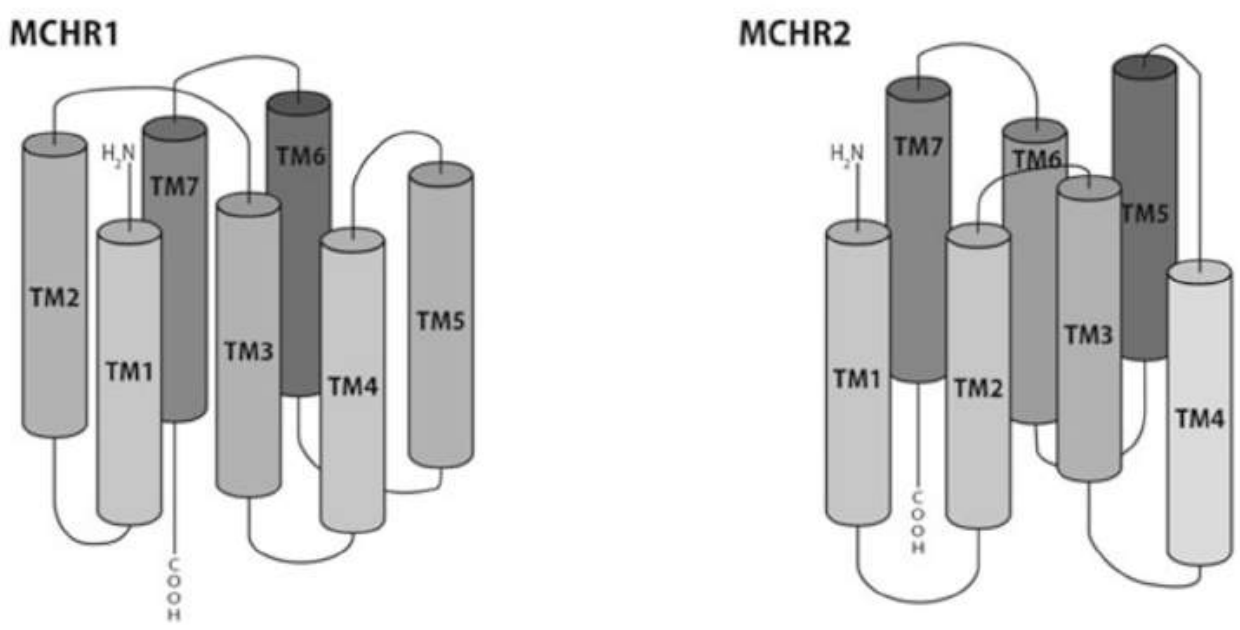

Fig. 2 Structure of the $M C H R I$ and $M C H R 2$ genes and their corresponding protein structure. (a) The MCHRl gene is composed of two exons and one intronic sequence (represented by broken lines, not to scale). MCHR2 displays considerably more genomic complexity and is formed by six exons and five intronic regions of variable lengths. Exons $1 \mathrm{a}$ and $1 \mathrm{~b}$ correspond to splice variants of this gene, with E1b coding for the active form of the receptor. (b) A simplified representation of the three-dimensional structure of these receptors. Portions indicated by "TM" correspond to transmembrane domains of the receptor (the membrane has been omitted for clarity)

genomic databases for homologous sequences. They identified a sequence coding for a putative GPCR that, despite an overall low identity, was more similar to MCHRI than to any other gene. This gene, now identified as human MCHR2 (Gene ID: 84539), is located on chromosome $6 \mathrm{q} 16.2$, spans over $23 \mathrm{~kb}$, and has six exons and five intronic sequences, displaying considerably more genomic complexity than MCHRI. The ORF of MCHR2 is 1023 bp long and encodes a 340 aa protein. The first two exons of MCHR2 are splice variants, with Exon $1 \mathrm{~b}$ coding for the "extended" version of $M C H R 2$ and Exon 1a coding for an MCHR2 truncated in the N-terminal portion. Exon $1 \mathrm{~b}$ encodes the first 60 aa of $M C H R 2$, Exon 2 encodes 70 aa, Exon 3 encodes 66 aa, Exon 4 encodes 40 aa, and Exon 5 encodes the final 104 aa. Exon 1a corresponds to a 24 aa sequence, resulting in a truncated version of MCHR2 that has 304 aa (Fig. 2). In addition to the characteristic 7-TM structure, MCHR2 also displays several other conserved properties of GPCRs, such as two N-linked glycosylation sites, a DRY motif located at the end of TM3, an Asp residue in TM3, an NPXXXY motif in TM7, and a potential palmitoylation site in the C-terminal region (Fig. 2).

As is the case with MCHR1, MCHR2 is highly selective for $\mathrm{MCH}$ as its ligand. Transfected cells respond to both mammalian and salmonid $\mathrm{MCH}$ in $\left[\mathrm{Ca}^{2+}\right]$ assays, 
with an $\mathrm{EC}_{50}$ that ranges from 0.085 to $31 \mathrm{nM}$, depending on the cell line used, the transfection stability, and the method used to measure $\mathrm{Ca}^{2+}$ mobilization. These $\mathrm{EC}_{50}$ values are similar in magnitude to the values of MCHR 1 in similar conditions, if not slightly lower. There is no response from MCHR2-transfected cells to other peptides of the MCH peptidergic system, such as NEI, NGE, and MGOP; to natriuretic peptides, such as rat ANP (1-28 and 3-28), human C-type natriuretic peptide-22, and human brain natriuretic peptide-32; to somatostatin and somatostatin-related peptides, such as somatostatin-14, somatostatin-28, cortistatin-14, and cortistatin29; to opioid ligands, such as $\gamma$-endorphin, dynorphin $\mathrm{A}$, and dynorphin $\mathrm{B}$; or to another member of the melanocortinergic system, $\alpha-\mathrm{MSH}$. No effect of $\mathrm{MCH}$ or any other peptide was observed for the truncated version of MCHR2.

A remarkable aspect of MCHR2 is its low similarity to MCHR1, despite their obvious paralogous relationship. The two proteins share a mere $38 \%$ identity at the aa level, a remarkably low amount for proteins of the same family that bind to the same ligand. Such a high level of divergence between two paralogues suggests that they appeared early in vertebrate evolution and that there was weak selective pressure on at least one of them. The presence of orthologues of both MCHRI (Gene ID: 103189252) and MCHR2 (Gene ID: 103178350) in the elephant shark (Callorhinchus milii) genome supports this early appearance of a family of receptors for $\mathrm{MCH}$. The low selective pressure on MCHR2 is supported by the loss of this receptor in the species of the Glires clade. In the rabbit (Oryctolagus cuniculus - order Lagomorpha), a base substitution in Exon 2 resulted in the transformation of a CGA codon (Gly ${ }^{85}$ in the human gene) to a TGA stop codon. In the guinea pig (Cavia porcellus - order Rodentia), a frameshift mutation occurred by the introduction of an additional A after bp 47, resulting in an early stop codon that truncated the protein. Rats, mice, and hamsters have no indication of an MCHR2 orthologue in their genomes, suggesting the removal of truncated sequences after the mutations that probably occurred in the last common ancestor of the Glires superorder (Tan et al. 2002). The lack of information regarding MCHR2 functions impairs, for now, our ability to speculate about what kind of environmental aspects could have loosened the evolutionary constraint on MCHR2, or even what gains, if any, the animals of the Glires clade could have obtained by losing this receptor.

\section{Morphological and Neurochemical Aspects of MCH/NEI Neurons}

The first complete description of MCH and NEI immunoreactivity in the mammalian brain using a specific antibody was published in 1992, using male animals of albino (Sprague Dawley) and pigmented (Long-Evans) rat strains (Bittencourt et al. 1992). A vast amount of work was invested from then on to expand our knowledge of the anatomical features of the $\mathrm{MCH}$ peptidergic system in other mammals. There are now available descriptions of the distribution of $\mathrm{MCH}$-ir neurons in the rat, mouse, Siberian hamster, Syrian hamster, tufted capuchin monkey (a New World primate), 
human, pig, sheep, and cat (as reviewed in Bittencourt 2011). The description of this peptidergic system in other physiological models, such as females in different reproductive stages (Knollema et al. 1992; Rondini et al. 2007, 2010; Alvisi et al. 2016), also contributed to a greater understanding of this system, but detailed studies regarding its sexual dimorphism are still lacking. In addition to its description in different mammals, several works also contributed to refining the distribution of this system, revealing new neurochemical markers associated with $\mathrm{MCH}$-containing cells, and unveiling the networks that integrate this peptidergic system into functional circuits. It is noteworthy, however, that the lack of systematization regarding anatomical nomenclature has hindered the field, with several different names used to refer to the same structures.

A considerable amount of information was gathered about $\mathrm{MCH}$ neurons in the last two decades. For example, it was demonstrated that $80-90 \%$ of MCH neurons also contain the mRNA coding for the glutamate decarboxylase (GAD67) enzyme, which suggests GABA synthesis in these cells (Rondini et al. 2007, 2010; Elias et al. 2008). Recent reports, however, suggest that a subset of MCH neurons may express the machinery for glutamate transport and release (Chee et al. 2015). A recent work further complicates our understanding of neurotransmitter use by $\mathrm{MCH}$ neurons, suggesting almost all of these neurons contain the machinery for GABA synthesis ( Gadl and Gad2) but lack the vesicular GABA transporter needed for the canonical pathway of GABA release (Slc32al) (Mickelsen et al. 2017). On the other hand, almost all of MCH neurons express the vesicular glutamate transporter 2 (Slc17a6), suggesting a glutamatergic phenotype for these neurons (Mickelsen et al. 2017). Neuronal $\mathrm{MCH}$ is also extensively colocalized with CART, ranging from $70 \%$ to 95\% colocalization depending on the region analyzed (Elias et al. 2001). There is also colocalization of MCH with nesfatin-1 (Brailoiu et al. 2007; Fort et al. 2008; Foo et al. 2008), neuronal pentraxin 1 (Reti et al. 2002), acetylcholinesterase in the absence of acetyltransferase (Risold et al. 1989; Chou et al. 2004), monocyte chemoattractant protein-1/CCL2 (Banisadr et al. 2005), secretogranins II and III (Tanabe et al. 2007; Hotta et al. 2009), and $\alpha$-dystrobrevin (Hazai et al. 2008).

$\mathrm{MCH}$ neurons also contain a myriad of receptors, which render them responsive to several inputs originating broadly in the CNS. These neurons receive both GABAergic and glutamatergic inputs, which are processed by the ionotropic $\mathrm{GABA}_{\mathrm{A}}$ (containing subunits $\alpha 2$ and $\alpha 3$ ), NMDA, and AMPA receptors and the metabotropic receptors mGlul and mGlu5 present in these cells (Bäckberg et al. 2004; van den Pol et al. 2004; Huang and van den Pol 2007). These neurons are also responsive to the neighboring $\mathrm{ORX}$ cells through the $\mathrm{ORXR}_{1}$ and $\mathrm{ORXR}_{2}$ receptors (van den Pol et al. 2004; Parks et al. 2014b). Melanin-concentrating hormone neurons are also responsive to endogenous opioids, probably through kappa-opioid and nociceptin/orphanin receptors (Parsons and Hirasawa 2011; Parks et al. 2014b). Other receptors present in $\mathrm{MCH}$ cells are the $\mathrm{V} 1 \mathrm{a}$ receptor for vasopressin and OXTR for oxytocin (Yao et al. 2012), NK3 for neurokinin B (Griffond et al. 1997), 5-HT 1 A for serotonin (Collin et al. 2002), KissR for kisspeptin, SSTR 1 and SSTR2 for somatostatin, NTSR1 for neurotensin, NPSR for neuropeptide S, CCKAR for cholecystokinin (Parks et al. 2014b), H3R for histamine (Parks et al. 


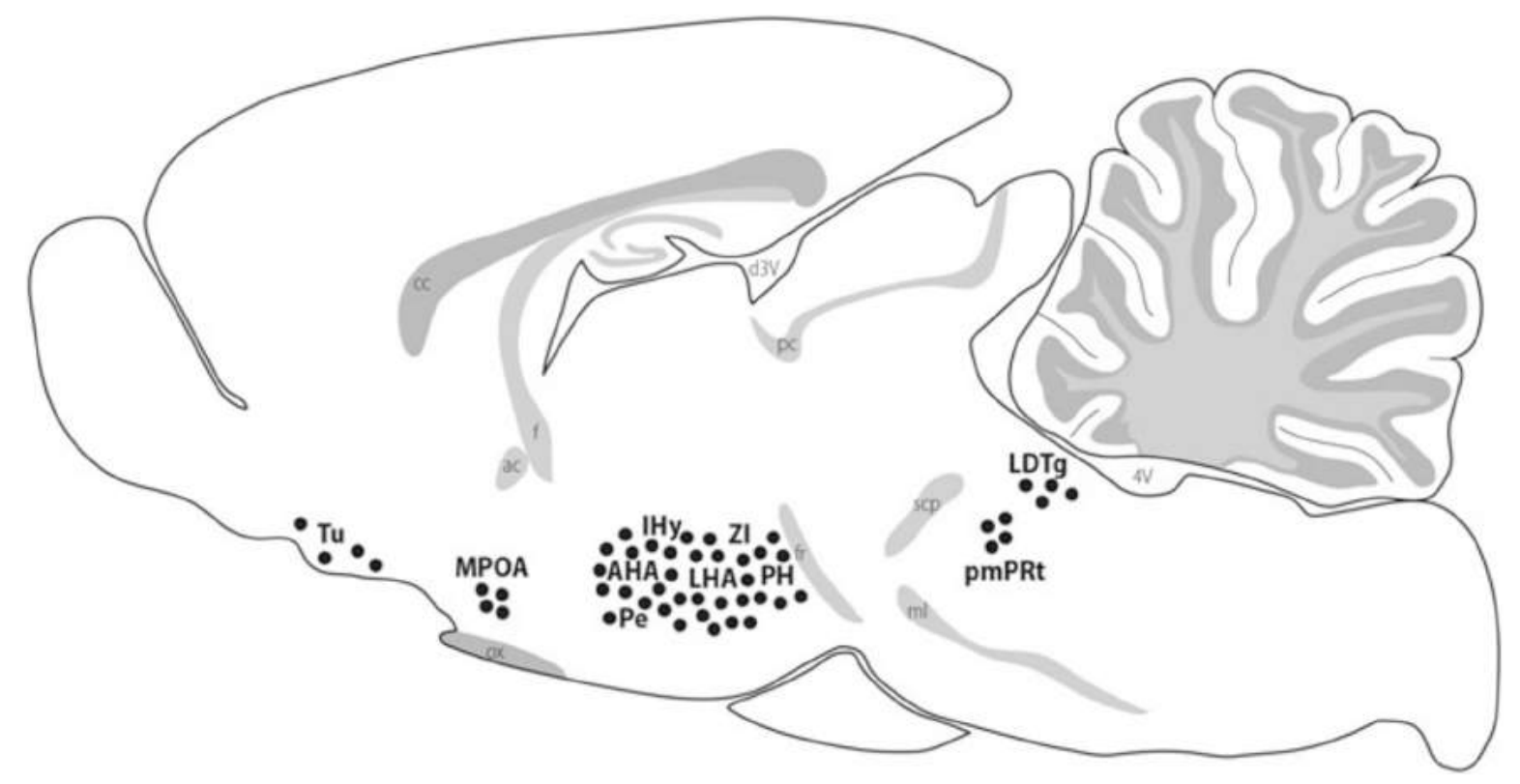

Fig. 3 The distribution of MCH-containing neurons in the rat neuroaxis. Schematic representation of a parasagittal slice of a rat brain. Significant fiber tracts are indicated in gray to provide reference points to the reader. In rostrocaudal order, the primary loci of $\mathrm{MCH}$ neurons are as follows: the olfactory tubercle (Tu), the medial preoptic area (MPOA) of lactating females, the incertohypothalamic area (IHy), the periventricular nucleus of the hypothalamus (Pe), the anterior hypothalamic area (AHA), the lateral hypothalamic area (LHA), the zona incerta (ZI), the posterior hypothalamic area ( $\mathrm{PH})$, the paramedian pontine reticular formation (pmPRt), and the laterodorsal tegmental nucleus (LDTg) only in females. Other abbreviations: $4 V$ fourth ventricle, ac anterior commissure, cc corpus callosum, $d 3 \mathrm{~V}$ dorsal third ventricle, $f$ fornix, fr fasciculus retroflexus, $m l$ medial lemniscus, ox optic chiasm, $p c$ posterior commissure, scp superior cerebellar peduncle

2014a), and the adrenergic receptor $\alpha_{2 \mathrm{~A}}$, which responds to both noradrenergic and dopaminergic inputs (Modirrousta et al. 2005; Alberto et al. 2011; Conductier et al. 2011). MCH neurons are also responsive to insulin through the insulin receptor (Hausen et al. 2016), as well as to glucose, probably through the GLUT3 glucose transporter (Burdakov et al. 2005; Kong et al. 2010).

In the following sections, the distribution of $\mathrm{MCH}$ along the rostrocaudal axis of the rat CNS will be described (Bittencourt et al. 1992), as this is the most detailed description available, and the pertinent parallels to what is known about the human MCH system will be drawn (Elias et al. 1998; Bittencourt 2011; Krolewski et al. 2010). A schematic representation of each area that will be discussed in the section is presented in Fig. 3. Since there is over 96\% colocalization between MCH and NEI in the rat brain (Bittencourt et al. 1992), all descriptions in this chapter are valid for both peptides, although we will refer only to "MCH" for the sake of simplicity. The peptide NGE will not be discussed in this section, as the scarcity of data renders impossible any discussion of its immunoreactivity in vivo. 


\subsection{Olfactory Tubercle}

The anterior-most group of MCH-ir cells is found in the olfactory tubercle (Tu), in the basal forebrain area, ranging from +0.45 to $-0.45 \mathrm{~mm}$ from bregma. These small and multipolar immunoreactive cells are found in the polymorph or multiform layer of the Tu (Fig. 4a-a'). A small contingent of cells is found bordering the islands of Calleja, a neighboring structure, while a few cells were found dorsally within the substantia innominata and caudally within the anterior amygdaloid area. This population is most easily labeled through immunohistochemistry in colchicinetreated animals or through in situ hybridization, suggesting that these cells have a low rate of $\mathrm{MCH}$ synthesis or a high rate of degradation. While $\mathrm{MCH}$-ir cells are found in both male and female rat brains, there is no evidence of this population in other species, including humans. It is uncertain, at this time, whether this $\mathrm{Tu}$ population is exclusive to rats or poorer immunogenicity in other species generates false negative results.

\subsection{Diencephalon}

\subsubsection{Medial Preoptic Area}

The medial preoptic area (MPOA) represents the most anterior group of Pmch mRNA-expressing and MCH-ir neurons in the diencephalon and is one of the two known areas where $\mathrm{MCH}$ is sexually dimorphic. While not observable in the male rat brain, in females during the estrous cycle or in pregnant females, Pmch mRNA can be detected in the MPOA of lactating rats as soon as the 5 th postpartum day. In addition to the mRNA expression, immunoreactive neurons can be identified in high numbers in the ventromedial aspects of the MPOA (Fig. 4b- $b^{\prime}$ ), with some of them invading the limits of the periventricular preoptic nucleus of the hypothalamus and the rostralmost parts of the paraventricular nucleus of the hypothalamus $(\mathrm{PVH})$ (Knollema et al. 1992) (Fig. 4c- $\mathrm{c}^{\prime}$ ). This population of neurons has a very specific temporal development pattern, with increasing synthesis of $\mathrm{MCH}$ as lactation progresses, reaching a peak between the 15th and 19th days of lactation and disappearing after weaning (Rondini et al. 2010; Alvisi et al. 2016). Recently, it has been demonstrated that $\mathrm{MCH}$ synthesis in this area is directly correlated to the postpartum, but not the prepartum, litter size (Ferreira et al. 2017b).

\subsubsection{Incerto-hypothalamic Area}

The first major group of MCH-ir and Pmch mRNA-expressing cells found in the neuraxis of both male and female animals is located at the level of the caudal PVH and was initially designated the rostromedial zona incerta (ZI) group. The designation of 

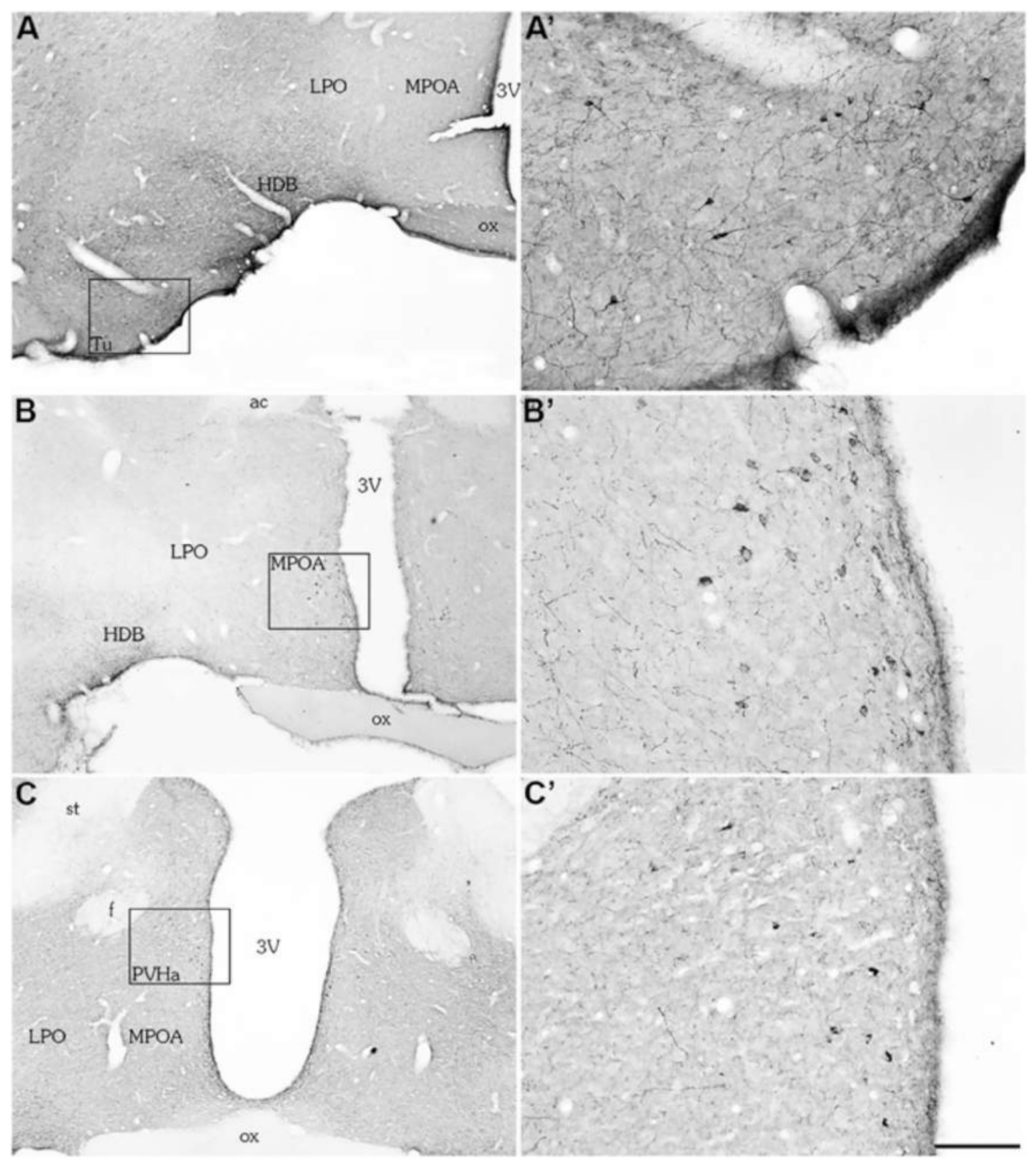

Fig. 4 Areas containing MCH-producing neurons in the rat CNS. Brightfield photomicrographs of frontal rat slices subjected to immunohistochemistry for $\mathrm{MCH}$ localization. (a) Small immunoreactive neurons are found in the olfactory tubercle (Tu) of rats of both sexes. (a') Higher magnification of the rectangle in a. Immunoreactive neurons in the Tu are smaller than hypothalamic neurons. (b) Neurons that synthesize MCH are detectable in the medial preoptic area (MPOA) of females exclusively during the lactation period. ( $\mathbf{b}^{\prime}$ ) Higher magnification of the rectangle in $\mathbf{b}$. Neurons in the MPOA are concentrated in the ventromedial part of this nucleus and are not as densely stained as neurons in other areas. (c) In addition to neurons in the MPOA, the anterior part of the paraventricular nucleus of the hypothalamus (PVHa) also contains $\mathrm{MCH}$-ir neurons in lactating animals. $\left(\mathbf{c}^{\prime}\right)$ Higher magnification of the rectangle in $\mathbf{c}$. Other abbreviations: $3 \mathrm{~V}$ third ventricle, $a c$ anterior commissure, $f$ fornix, $H D B$ horizontal limb of the diagonal band of Broca, $\angle P O A$ lateral preoptic area, ox optic chiasm, st stria terminalis. Scale bar: $\mathbf{a}-\mathbf{c}, 500 \mu \mathrm{m} ; \mathbf{a}^{\prime}-\mathbf{c}^{\prime}$, $100 \mu \mathrm{m}$ 
this area was, perhaps, the most controversial involving $\mathrm{MCH}$ neurons, as the same area has been referred to as the rostromedial zona incerta, medial zona incerta, and rostral zona incerta by different authors, owing to the fact that it is not charted in most atlases. It was not until 2003, when an extensive neurochemical and hodological study was performed, that the area was properly renamed the incerto-hypothalamic area (IHy). Although this region can be considered a subthalamic region from a strictly anatomical standpoint, its hodology, neurochemistry, and cytoarchitecture indicate that this area is better described as a hypothalamic transitional region.

Morphologically, the IHy is found at the dorsoventral level of the tip of the third ventricle $(3 \mathrm{~V})$, and on the lateromedial axis, it is centered around an imaginary line drawn over the mammillothalamic tract $(\mathrm{mt})$, laterally limited by a line drawn over the fornix (f) (Fig. 5a-a'). The IHy can be identified, in cytological staining, as an ovoid-shaped area of five or six layers of cells that display a preferred lateromedial orientation. Neurochemically, this area is characterized by the presence of $\mathrm{MCH}-$ and CART-ir neurons intermingled with dopaminergic neurons (immunoreactive for tyrosine hydroxylase-TH-ir). These dopaminergic neurons are part of the A13 group, which partially overlaps with the IHy and the rostral zona incerta proper. Although MCH/CART-ir neurons are found in great proximity to $\mathrm{TH}$-ir neurons, there is no colocalization between these markers, and the relationship between these chemically distinct populations is yet to be explored in the literature. The IHy can be found in both rat and human samples of both sexes.

\subsubsection{Anterior Periventricular Nucleus}

At the caudal level of the PVH, another smaller population of $\mathrm{MCH}$-ir neurons inhabits the periventricular nucleus of the hypothalamus. These cells are preferentially distributed along the dorsal part of this nucleus (Fig. 5b-b'), sometimes constituting a cap-like structure at the top of the third ventricle $(3 \mathrm{Vdc})$ (Fig. $5 \mathrm{c}-$ $\left.c^{\prime}\right)$. This population is typically composed of elongated neurons, some oriented parallel to the $3 \mathrm{~V}$ wall and some radially oriented with respect to the ventricle, particularly on the $3 \mathrm{Vdc}$. This group of cells is stained in both male and female rat brains, but it cannot be seen in the mouse brain. There is also no evidence of this population in the human brain.

\subsubsection{Medial Hypothalamus}

Two populations of neurons can be identified in the medial zone of the hypothalamus. The first is a population of neurons that are caudal to the posterior part of the PVH, although never within its borders. This population predominantly occupies the caudal aspect of the anterior hypothalamic area (AHA) but extends beyond its borders to the medial hypothalamus rostral to the dorsomedial nucleus. The rostrocaudal distribution of this population partially overlaps those of the IHy and the anterior periventricular populations, but these neurons can be distinguished easily from the periventricular 

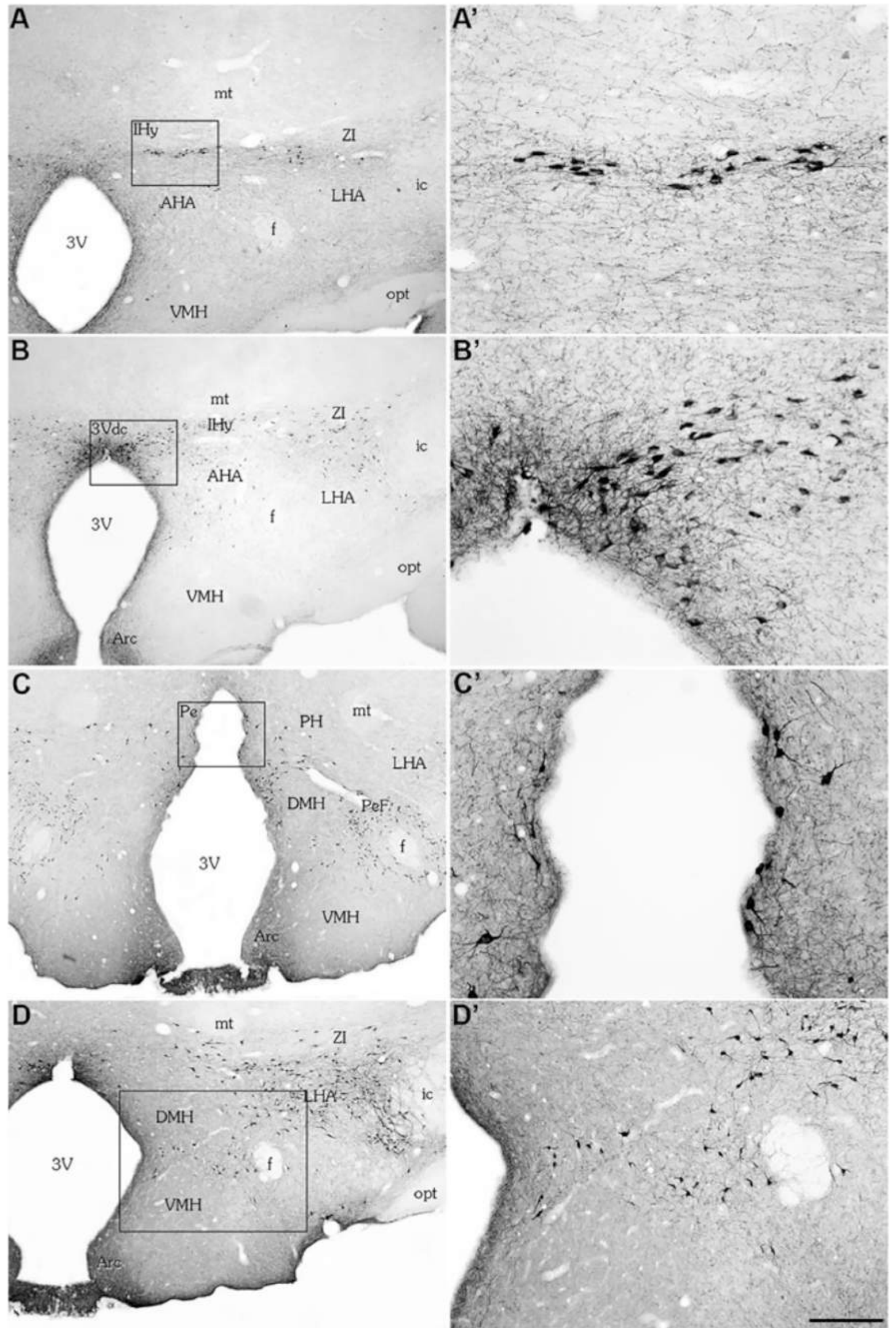

Fig. 5 Areas containing MCH-producing neurons in the rat CNS, continued. Brightfield photomicrographs of frontal rat slices subjected to immunohistochemistry for $\mathrm{MCH}$ localization. (a) In the diencephalon of rats of both sexes, $\mathrm{MCH}$-ir neurons are found in the incertohypothalamic area 
group as there is a gap between these two populations. The separation between the IHy and the medial hypothalamic group is harder to visualize, as these populations appear continuous in the dorsoventral axis. Melanin-concentrating neurons of the AHA, however, can be separated from the IHy by their distinct orientation: while IHy neurons are mostly bipolar and elongated in the lateromedial axis, AHA neurons are multipolar and without clear directionality. Although some neurons are found in the neuropil surrounding the ventral aspect of the PVH or the dorsal margin of the ventromedial nucleus, these neurons are never found within the limit of these nuclei.

A second population of MCH-ir neurons is found more caudally in the medial hypothalamus, at the level of the dorsomedial and ventromedial nuclei. These neurons are found outside the margins of these two nuclei, occupying the area between them (called the internuclear area) and forming a thin layer of cells stretching from the fornix to the periventricular nucleus (Fig. $5 \mathrm{~d}_{-}-\mathrm{d}^{\prime}$ ). Both medial hypothalamus populations are found in male and female rats, as well as in human brains.

\subsubsection{Lateral Hypothalamic Area}

The LHA is the main site in the hypothalamus that harbors $\mathrm{MCH}$-containing cells. Following the classical division of the LHA into three rostrocaudal subdivisions, i.e., anterior, tuberal, and posterior (Saper et al. 1979), MCH-ir cells are mostly found in the tuberal and posterior divisions of this structure. Owing to the size and complexity of this area, three subgroups of $\mathrm{MCH}$-ir neurons can be identified. The first group consists of immunoreactive neurons that occupy the space between the internal capsule (ic) and the fornix (f) in the lateromedial axis and the space between the ventral margin of the ZI and the level of the fornix (Fig. 6a-a' ${ }^{\prime}$. Neurons in this group are strongly labeled, medium to large and multipolar, giving rise to two to five

Fig. 5 (continued) (IHy). The IHy can be identified as a strip of MCH neurons between the third ventricle $(3 \mathrm{~V})$ and the mammillothalamic tract $(\mathrm{mt})$, at the dorsoventral level of the zona incerta (ZI). ( $\left.\mathbf{a}^{\prime}\right)$ Higher magnification of the rectangle in a. Neurons in the IHy are bipolar and frequently oriented in the lateromedial axis. (b) A distinctive set of neurons can be identified in the area surrounding the dorsal tip of the $3 \mathrm{~V}$, forming a cap of neurons ( $3 \mathrm{Vdc})$. $\left(\mathbf{b}^{\prime}\right)$ Higher magnification of the rectangle in $\mathbf{b}$. Neurons in the $3 \mathrm{Vdc}$ are often radially oriented with respect to the $3 \mathrm{~V}$. (c) Immunoreactive neurons also occupy the periventricular nucleus of the hypothalamus (Pe) along its tuberal extension. $\left(\mathbf{c}^{\prime}\right)$ Higher magnification of the rectangle in $\mathbf{c}$. Labeled cells in the Pe are found parallel or radially oriented with respect to the $3 \mathrm{~V}$ and extend projections to the subventricular zone. (d) Several MCH-ir neurons are found in the medial zone of the hypothalamus. At the level of the dorsal $(\mathrm{DMH})$ and ventromedial $(\mathrm{VMH})$ hypothalamic nuclei, these neurons are found along the margin of those nuclei, in the internuclear area. ( $\mathbf{d}^{\prime}$ ) Higher magnification of the rectangle in $\mathbf{d}$. The internuclear group of $\mathrm{MCH}$ neurons extends itself from the fornix (f) to the $3 \mathrm{~V}$. Other abbreviations: AHA anterior hypothalamic area, Arc arcuate nucleus, $i c$ internal capsule, LHA lateral hypothalamic area, opt optic tract, PeF perifornical nucleus. Scale bar: a-d, $500 \mu \mathrm{m} ; \mathbf{d}^{\prime}, 200 \mu \mathrm{m}$; and $\mathbf{a}^{\prime}-\mathbf{c}^{\prime}$, $100 \mu \mathrm{m}$ 


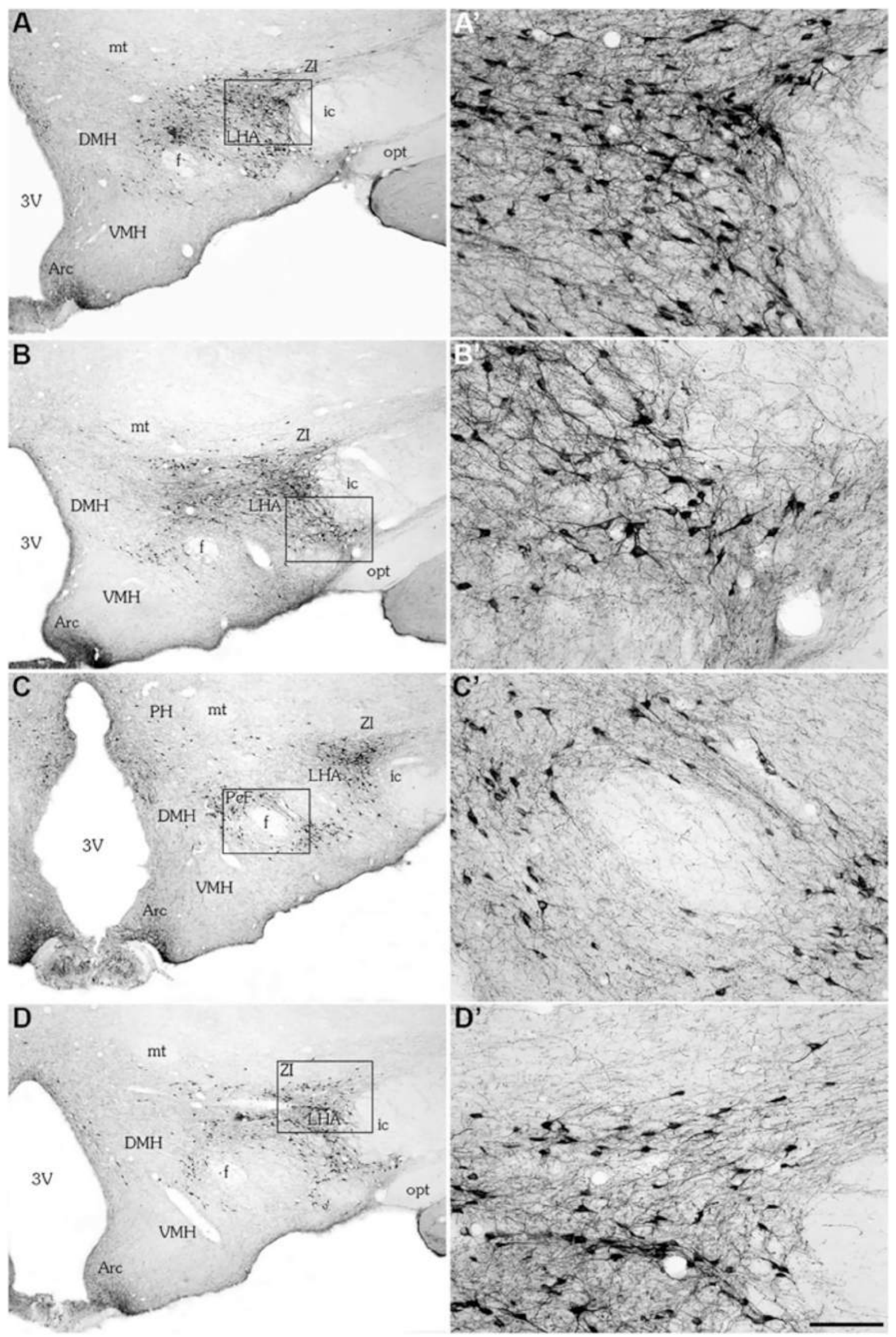

Fig. 6 Areas containing MCH-producing neurons in the rat CNS, continued. Brightfield photomicrographs of frontal rat slices subjected to immunohistochemistry for $\mathrm{MCH}$ localization. The most 
primary dendrites. Secondary dendritic branching is frequently observed, although tertiary branching is less common. These neurons form the bulk of the MCH-ir neurons in the anterior tuberal hypothalamus, and in certain cases, they appear to be continuous with the stained neurons of the medial hypothalamus. The second group is formed by very large immunoreactive neurons, located in the region dorsal to the optic tract (opt) and ventromedial to the tip of the internal capsule (Fig. 6b-b'). Owing to the unique size of these neurons, this area is called the magnocellular group of the LHA, and it is more evident at the levels at which both ventromedial and dorsomedial nuclei are also present. Third, as the LHA progresses in the rostrocaudal axis, a group of $\mathrm{MCH}$-ir neurons is found surrounding the fornix, in the perifornical nucleus (Fig. 6c$\mathrm{c}^{\prime}$ ). This population is intermingled at more rostral levels with the group that spans the space between the fornix and the internal capsule, which makes it somewhat hard to isolate. At more caudal levels, it becomes better delineated as the number of $\mathrm{MCH}$-ir neurons medial to the internal capsule decreased owing to the increased presence of white matter patches of the $m f b$. It is noteworthy that some authors believe there to be a fourth population, located dorsally to the peduncular part of the lateral hypothalamus and lateral to the subincertal nucleus, called the DL-PLH. In all likelihood, this population is composed of LHA neurons that are pushed to the dorsal part of the internal capsule as the $m f b$ expands into the LHA.

In humans, the distribution of $\mathrm{MCH}$ in the LHA is similar to that of the rat, but not identical, owing to the smaller lateromedial extent of the hypothalamus in primates as a result of the expansion of the internal capsule. Therefore, a large contingent of immunoreactive neurons is found medial to the fornix, in the space between the fornix and the third ventricle, although some immunoreactive neurons are still found intermingled with fibers of the internal capsule. There are no known sex-related differences in the LHA MCH population.

Fig. 6 (continued) densely populated MCH-ir area in the rat CNS is the lateral hypothalamic area (LHA). Due to its complexity, the MCH neurons in this area can be divided into subgroups. (a) The main subgroup of MCH-ir neurons is found in the dorsomedial part of the LHA, ventral to the zona incerta ( $\mathrm{ZI}$ ), and medial to the internal capsule (ic), extending approximately to the level of the fornix (f). ( $\left.\mathbf{a}^{\prime}\right)$ Higher magnification of the rectangle in a. Neurons in the LHA are large, densely stained, multipolar, and without clear orientation. Projections from these neurons can be seen intermingled with the white matter patches of the medial forebrain bundle $(\mathrm{mfb})$. (b) In the area immediately ventromedial to the ic and dorsal to the optic tract (opt), several very large immunostained neurons can be found, corresponding to the magnocellular group of the LHA. $\left(\mathbf{b}^{\prime}\right)$ Higher magnification of the rectangle in $\mathbf{b}$. (c) Neurons immunoreactive to $\mathrm{MCH}$ are also found encircling the fornix, in the perifornical nucleus $(\mathrm{PeF})$. This group is more easily identified at caudal levels of the tuberal hypothalamus. $\left(\mathbf{c}^{\prime}\right)$ Higher magnification of the rectangle in $\mathbf{c}$, showing the concentric distribution of MCH-ir neurons around the fornix. (d) In close proximity to the LHA, a small number of $\mathrm{MCH}$-ir neurons can be labeled in the ZI. (d' $\left.\mathbf{d}^{\prime}\right)$ Higher magnification of the rectangle in $\mathbf{d}$. Despite the spatial proximity, an apparently "empty" band of neuropil separates the ZI and the LHA neurons. Other abbreviations: $3 \mathrm{~V}$ third ventricle, $A r c$ arcuate nucleus, $D M H$ dorsomedial hypothalamic nucleus, $m t$ mammillothalamic tract, $\mathrm{PH}$ posterior hypothalamic area, $V M H$ ventromedial hypothalamic nucleus. Scale bar: a-d, $500 \mu \mathrm{m} ; \mathbf{a}^{\prime}-\mathbf{d}^{\prime}, 100 \mu \mathrm{m}$ 


\subsubsection{Zona Incerta}

Although MCH-ir neurons do not occupy the rostral ZI, a population of these neurons can indeed be found in the lateral ZI. These neurons occupy the area immediately dorsal to the LHA, forming a three- to four-cell-thick layer in the stratum over the dorsal part of the internal capsule, mostly at the rostrocaudal level of the dorsomedial and ventromedial nuclei (Fig. $\left.6 \mathrm{~d}-\mathrm{d}^{\prime}\right)$. Although there is conspicuous proximity between the ZI and the LHA groups, an apparently "empty" band of neuropil separates these two populations. Furthermore, neurons in the ZI are large and multipolar, but their major axis is oriented horizontally, following the overall shape of the area, while LHA neurons have no distinct polarity. Lateromedially, incertal neurons do not go more than halfway between the internal capsule and the fornix. Neurons that synthesize MCH are present in the ZI of rats and humans, regardless of sex.

\subsubsection{Tuberomammillary Nucleus, Dorsomedial Part}

The dorsomedial part of the tuberomammillary nucleus (TMdm) was one of the unexpected $\mathrm{MCH}$-containing cell groups described during the discovery of $\mathrm{MCH}$ in the mammalian brain. This nucleus is localized laterodorsally on a diagonal axis between the brain surface at the level of the ventral premammillary nucleus toward the tip of the third ventricle, where it begins to divide and disappear posteriorly, and its ventral part becomes the mammillary recess (Fig. 7a-a'). This group of $\mathrm{MCH}$-containing cells is intermingled with histaminergic cells, although there is no colocalization between these two neuroactive substances (Casatti et al. 2002). It must be noted that this group of cells is not the tuberal magnocellular nucleus of Bleier (Bleier et al. 1979), as the proper tuberal magnocellular nucleus is localized ventrally, forming a sheet of cells dorsal to the pial surface of the brain at the level of the supramammillary nucleus. This group of cells can be found in both male and female rat brains. No specific mention of this nucleus has been made in studies of the primate brain.

\subsubsection{Posterior Hypothalamus}

A few scattered immunoreactive neurons are found in the posterior hypothalamic area $(\mathrm{PH})$. These neurons represent the caudal limit of the $\mathrm{MCH}$ distribution and have a similar rostrocaudal level to the tuberomammillary nucleus immunoreactive cells (Fig. 7a). In the human brain, likewise, $\mathrm{MCH}$-ir neurons were found extending back into the posterior hypothalamic area. There are no known sex-related differences in this population. 

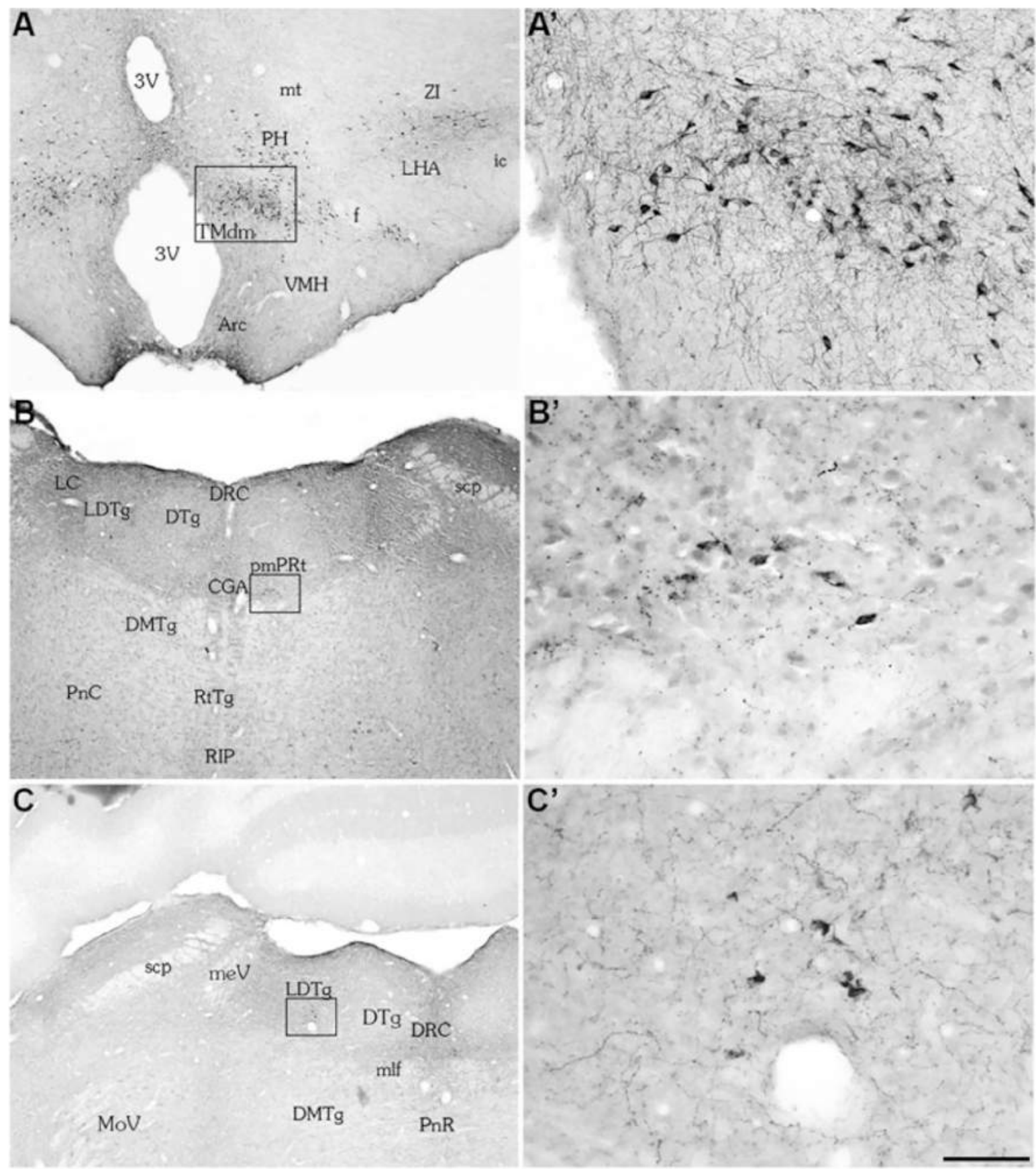

Fig. 7 Areas containing MCH-producing neurons in the rat CNS, continued. Brightfield photomicrographs of frontal rat brain slices subjected to immunohistochemistry for $\mathrm{MCH}$ localization. (a) At the caudal limit of the hypothalamus, at the transition between the tuberal, and at mammillary subdivisions, the last $\mathrm{MCH}$-ir neurons in the hypothalamus are found. Neurons can be identified in the posterior hypothalamic area (PH) and lateral hypothalamic area (LHA) and in great numbers in the tuberomammillary dorsal nucleus (TMdm). ( $\left.\mathbf{a}^{\prime}\right)$ Higher magnification of the rectangle in a, highlighting the labeled neurons in the TMdm. (b) In the male brainstem, $\mathrm{MCH}$-ir neurons are restricted to the paramedian pontine reticular formation (pmPRt), located adjacent to the $\alpha$ part of the central gray (CGA), and ventromedial to the dorsal tegmental nucleus (DTg). ( $\mathbf{b}^{\prime}$ ) Higher magnification of the rectangle in $\mathbf{b}$, highlighting labeled neurons in the pmPRt. (c) Labeled neurons are found in the laterodorsal tegmental nucleus (LDTg) exclusively in female rats, regardless of reproductive stage. $\left(\mathbf{c}^{\prime}\right)$ Higher magnification of the rectangle in $\mathbf{c}$. Other abbreviations: $3 \mathrm{~V}$ third ventricle, $\operatorname{Arc}$ arcuate nucleus, $D M T g$ dorsomedial tegmental area, $D R C$ caudal part of the dorsal raphe nucleus, $f$ fornix, $i c$ internal capsule, $L C$ locus coeruleus, LHA lateral hypothalamic area, meV mesencephalic V tract, mlf medial longitudinal fasciculus, MoV motor nucleus of the $\mathrm{V}$ nerve, $m t$ 


\subsection{Paramedian Pontine Reticular Formation}

During the original anatomical characterization of $\mathrm{MCH}$ in the male rat, an unexpected group of MCH-containing cells was found in the reticular formation, in an area designated the paramedian pontine reticular formation ( $\mathrm{pmPRt}$ ). These neurons can be seen intermingled with the fibers that constitute the superior cerebellar peduncle at the level of the caudal part of the laterodorsal tegmental nucleus, with some of these neurons located dorsally between the borders of the dorsal raphe nucleus and the pontine central gray matter (Fig. 7b-b'). So far, there has been no further neurochemical characterization of these neurons, with no description regarding other neuropeptides or classical neurotransmitters they may synthesize. This group of cells can be found in both male and female rat brains, but it has never been reported in humans.

\subsection{Laterodorsal Tegmental Nucleus}

The laterodorsal tegmental nucleus (LDT) was the second nucleus in the rat CNS to be discovered as sexually dimorphic regarding Pmch mRNA expression and peptide synthesis (Rondini et al. 2007). Labeling in this nucleus is observed only in female rats, regardless of reproductive status (Fig. $7 \mathrm{c}-\mathrm{c}^{\prime}$ ). These $\mathrm{MCH}$ neurons are also GABAergic, as revealed by their co-expression of GAD67-encoding mRNA, but not cholinergic, despite a significant presence of cholinergic neurons in this nucleus. In addition to the LDT, some neurons can be found in the adjoining subcoeruleus/ sublaterodorsal nucleus (SDL). Since both LDT and SDL have been implicated in rapid eye movement (REM) sleep (Garcia et al. 2017), this group of MCH neurons may be involved in the particular features of female REM sleep.

\section{The Projections of MCH Neurons}

The MCH- and NEI-ir neurons project widely to the CNS, to the point that "every commonly recognized cell group and cortical field in the rat brain was found to contain at least some MCH- and NEI-ir fibers" (Bittencourt et al. 1992). As a complete description of every projection would be too exhaustive, we will simply highlight the general organization of these fibers, and a more detailed description of

Fig. 7 (continued) mammillothalamic area, $P H$ anterior hypothalamic area, $P n C$ caudal part of the pontine reticular nucleus, $P n R$ pontine raphe nucleus, $R t T g$ reticulotegmental nucleus of the pons, $R I P$ raphe interpositus nucleus, $s c p$ superior cerebellar peduncle, $V M H$ ventromedial hypothalamic nucleus. Scale bar: a-c, $500 \mu \mathrm{m} ; \mathbf{a}^{\prime}, 100 \mu \mathrm{m} ;$ and $\mathbf{b}^{\prime}-\mathbf{c}^{\prime}, 50 \mu \mathrm{m}$ 
projections to sleep-related nuclei will be provided in the next chapter. As was the case with the cellular distribution, there is enough similarity between the patterns of $\mathrm{MCH}$ and NEI staining that these two can be used interchangeably. The projections of $\mathrm{MCH}$-ir neurons can be organized into four major pathways: ascending, dorsomedial periventricular, pituitary, and descending, in addition to lateral projections local to the hypothalamus. Figure 8 is a schematic representation of all four pathways. Each pathway will be described in this section.

\subsection{Ascending Pathway}

The major fiber path taken by the $\mathrm{MCH}$-ir fibers is the $m f b$, reinforcing the intimate connection of this complex fiber bundle to the LHA and its neurons. In frontal slices of the LHA, immunoreactive fibers can be seen occupying a large area of the $m f b$, with a special concentration in the ventromedial aspect of compartment $c$ of this bundle (Nieuwenhuys et al. 1982). As the $m f b$ ascends to the rostral portion of the brain, the first branching of $\mathrm{MCH}-i$ fibers occurs, with these projections exiting laterally through the ansa peduncularis to reach the amygdaloid complex, the basal nuclei, and the substantia innominata. Some of these fibers may also cross the subcortical structures to reach the external capsule and innervate the lateral portion of the cortical mantle. At the level of the preoptic area, fibers coursing through the mfb enter the stria medullaris, destined for the habenular nuclei, and others enter the stria terminalis to complement innervation of the amygdaloid complex. At the level of the preoptic recess, a major split occurs in the $m f b$-traversing $\mathrm{MCH}$-ir fibers: the majority, occupying the most medial aspect of the nucleus of the diagonal band, bend dorsomedially and spread to reach several cortical areas, the septal region, and the hippocampal formation, the latter in close association with the fornix. A smaller contingent of fibers, on the other hand, exits the $m f b$ and travels through the anterior commissure to reach the anterior olfactory area and the olfactory bulbs.

There is ample innervation of the cortical mantle by $\mathrm{MCH}$-ir fibers (Bittencourt et al. 1992; Saper et al. 1986; Cvetkovic et al. 2003; Elias et al. 2008). Among the immunoreactivity-rich fields are the infralimbic, prelimbic, anterior cingulate, and agranular insular cortices, with fewer fibers found in the somatosensory, auditory, and visual cortices. The claustrum is one of the most densely innervated cortical regions. The motor cortex also receives significant input through the $\mathrm{MCH}$-ir fibers (Fig. 9a-a'), most of which originate from neurons in the LHA (Elias et al. 2008). There is a laminar distribution of MCH-ir fibers, with layers I and IV containing more fibers, while a moderate density is observed in layers II and Vb. Layers III, IV, and $\mathrm{Va}$ are the most sparsely innervated. A substantial number of immunoreactive fibers are found in olfactory-linked areas, such as the main olfactory bulbs, the anterior olfactory nucleus, the olfactory tubercle, the piriform cortex, and the taenia tecta.

Many subcortical areas are also innervated by $\mathrm{MCH}$-ir fibers. The septal area contains some of the highest densities of immunoreactive fibers, including both en 


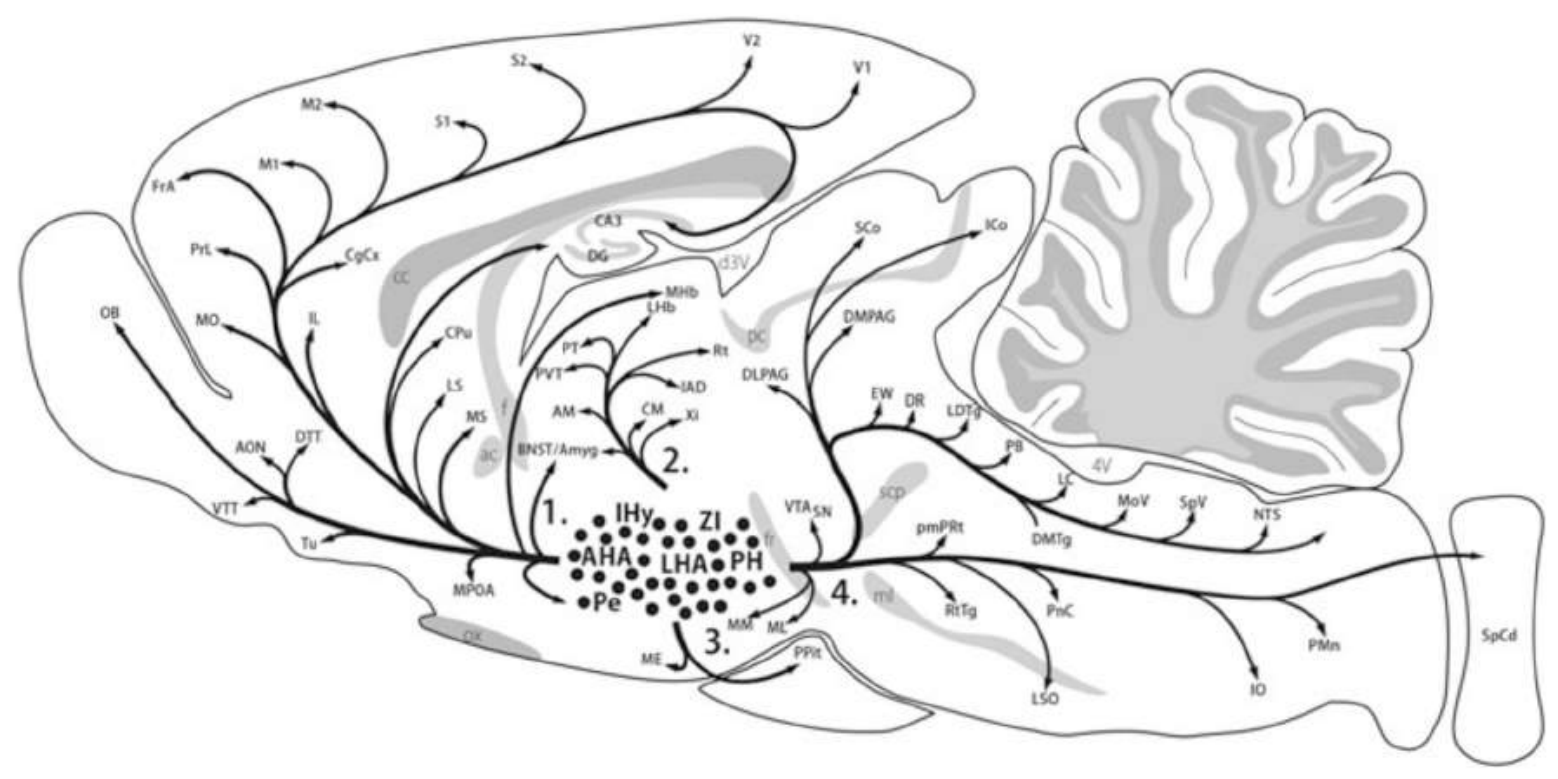

Fig. 8 The main pathways of MCH-ir fibers in the rat neuroaxis. Schematic representation of a parasagittal slice of a rat brain. Major fiber tracts are indicated in gray to provide reference points to the reader. There are four main projection pathways of $\mathrm{MCH}$-ir fibers in the rat brain: (1) The ascending pathway; (2) the periventricular dorsomedial pathway; (3) the pituitary pathway; and (4) the descending pathway. Abbreviations: $4 \mathrm{~V}$ fourth ventricle, ac anterior commissure, $A M$ anteromedial thalamic nucleus, Amyg amygdaloid complex, $A O N$ anterior olfactory nucleus, BNST bed nucleus of the stria terminalis, CA3 cornu ammonis 3 field of the hippocampus proper, cc corpus callosum, $C g C x$ cingulate cortex, $C M$ centromedial thalamic nucleus, $C P u$ caudate nucleus and putamen, $d 3 \mathrm{~V}$ dorsal third ventricle, $D G$ dentate gyrus, DLPAG dorsolateral periaqueductal gray matter, $D M P A G$ dorsomedial periaqueductal gray matter, $D M T g$ dorsomedial tegmental area, DR dorsal raphe nucleus, DTT dorsal taenia tecta, EW Edinger-Westphal nucleus, $f$ fornix, fr fasciculus retroflexus, FrA frontal association cortex, IAD interanterodorsal thalamic nucleus, ICo inferior colliculus, IL infralimbic, IO inferior olive, LC locus coeruleus, LDTg laterodorsal tegmental nucleus, $L H b$ lateral habenular nucleus, $L S$ lateral septal nucleus, $L S O$ lateral superior olive, $M 1$ primary motor cortex, $M 2$ secondary motor cortex, $M E$ median eminence, $M H b$ medial habenular nucleus, $M L$ lateral mammillary nucleus, $m l$ medial lemniscus, $M M$ medial mammillary nucleus, $M O$ medial orbital cortex, $M O V$ motor nucleus of the $\mathrm{V}$ nerve, $M P O A$ medial preoptic area, $M S$ medial septal nucleus, NTS nucleus of the solitary tract, $O B$ olfactory bulb, $O x$ optic chiasm, $P B$ parabrachial nucleus, $p c$ posterior commissure, $P H$ posterior hypothalamic area, $P M n$ paramedian reticular nucleus, pmPRt paramedian pontine reticular formation, PPit posterior pituitary, $\operatorname{PrL}$ prelimbic cortex, $P T$ paratenial thalamic nucleus, $P V T$ paraventricular thalamic nucleus, Rt reticular nucleus, $R t \operatorname{Tg}$ reticulotegmental nucleus of the pons, $S 1$ primary somatosensory cortex, $S 2$ secondary somatosensory cortex, SCo superior colliculus, scp superior cerebellar peduncle, $S N$ substantia nigra, $S p C d$ spinal cord, $S p V$ spinal trigeminal nucleus, $T u$ olfactory tubercle, $V 1$ primary visual cortex, $V 2$ secondary visual cortex, VTA ventral tegmental area, VTT ventral taenia tecta, $\mathrm{Xi}$ xiphoid thalamic nucleus

passant fibers and terminal boutons. Fibers are found at very high density in the medial nucleus and the diagonal band of Broca and at moderate to high density in the lateral nuclei, the bed nucleus of the stria terminalis, and the septofimbrial and triangular nuclei (Fig. 9b-c'). The hippocampal formation proper also receives a high density of fibers, including all cornu ammonis (CA) subfields, although there is greater fiber density in the dorsal hippocampal formation than in the ventral part, as 

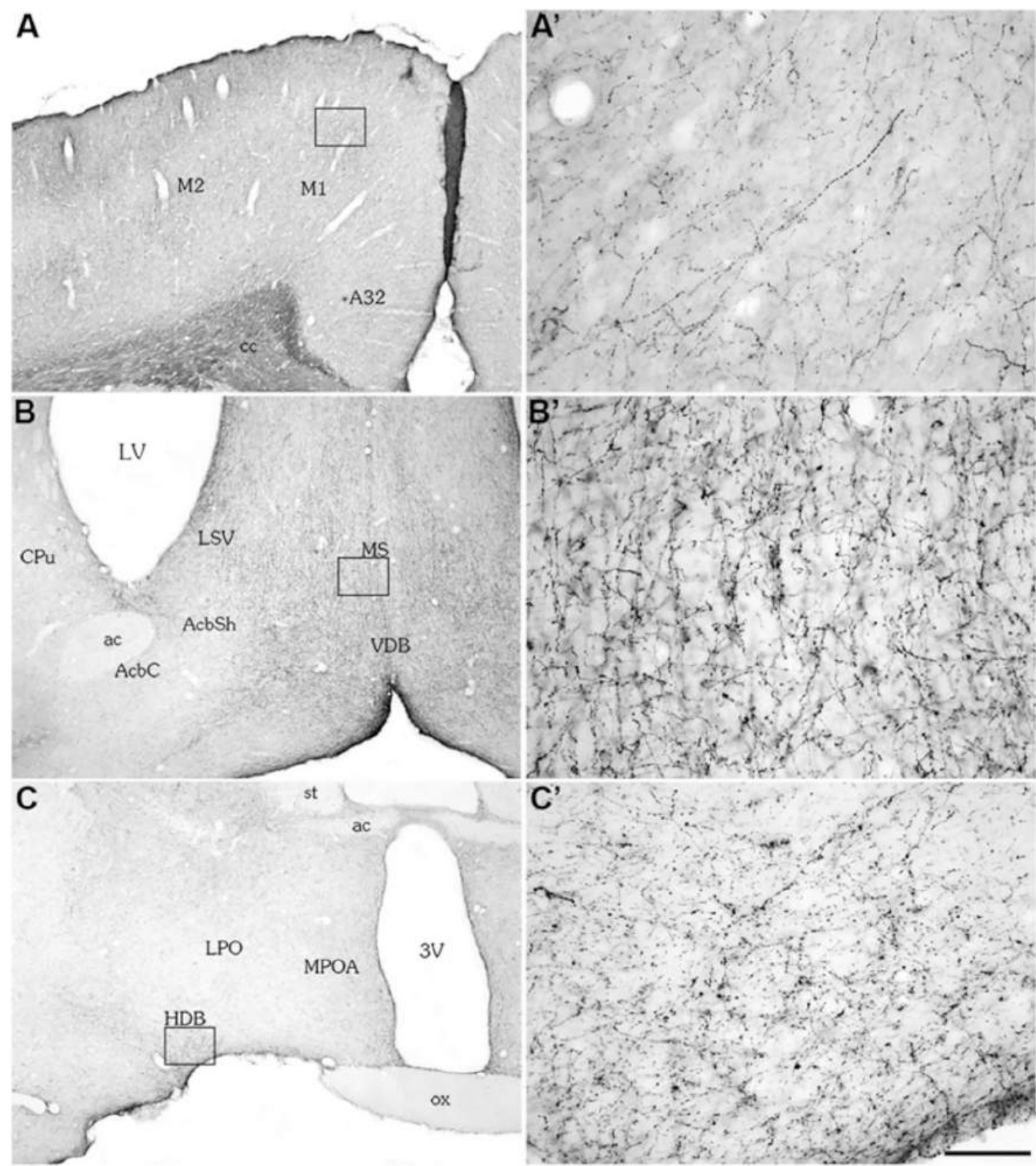

Fig. 9 The distribution of MCH-ir fibers in the rat neuroaxis. Brightfield photomicrographs of frontal rat slices subjected to immunohistochemistry for MCH localization. (a) Several cortical fields are innervated by MCH neurons, including the primary motor cortex (M1). (a') Higher magnification of the rectangle in $\mathbf{a}$, highlighting a long and varicose $\mathrm{MCH}$-ir fiber. (b) The medial septal nucleus (MS) is one of the densest areas of MCH innervation in the rat brain. ( $\mathbf{b}^{\prime}$ ) Higher magnification of $\mathbf{b}$. Numerous en passant fibers and terminal boutons can be found in the MS. (c) The horizontal limb of the diagonal band of Broca also contains a medium degree of innervation. $\left(\mathbf{c}^{\prime}\right)$ Higher magnification of the rectangle in $\mathbf{c}$. Numerous terminal boutons are found in the HDB. Other abbreviations: $3 V$ third ventricle, $A 32$ area 32 of the cingulate cortex, $a c$ anterior commissure, $A c b C$ core subdivision of the nucleus accumbens, AcbSh shell subdivision of the nucleus accumbens, cc corpus callosum, $C P u$ caudate nucleus and putamen, LPO lateral preoptic area, $L S V$ ventral part of the lateral septal nucleus, $M 2$ secondary motor cortex, $M P O A$ medial preoptic area, ox optic chiasm, st stria terminalis, VDB vertical limb of the diagonal band of Broca. Scale bar: a-c, $500 \mu \mathrm{m} ; \mathbf{a}^{\prime}-\mathbf{c}^{\prime}, 50 \mu \mathrm{m}$ 
well as a preferential localization of fibers in CA3 (Lima et al. 2013) (Fig. 10a). These projections to CA3 may be linked to memory retrieval in food-seeking tasks, as suggested by Sita et al. (2016). The dentate gyrus, on the other hand, receives low to moderate innervation, although in some cases immunoreactive fibers can be observed delineating the subgranular zone. The amygdaloid complex, including the medial, cortical, central, lateral, basolateral, basomedial, and intercalated nuclei, receives moderate innervation. Regarding the basal nuclei, there is a moderate to high density of fibers in the globus pallidus and the fundus of the striatum, a moderate to low number of fibers in the caudate-putamen nucleus and a low contingent of fibers in the nucleus accumbens. In the latter, fibers are found concentrated in the dorsomedial portion, close to the tip of the third ventricle, in the area identified as the septal pole (Haemmerle et al. 2015) (Fig. 10b-- b'). Kampe et al. (2009) demonstrated the existence of $\mathrm{MCH}$ neurons in the LHA that project simultaneously to the nucleus accumbens and the cingulate and insular cortices, suggesting that $\mathrm{MCH}$ may participate in a corticomesolimbic integration circuit.

\subsection{Dorsomedial Periventricular and Pituitary Pathways}

Immunoreactive fibers are found at moderate density in the hypothalamic periventricular zone, where they extend from the tuberal to the anterior preoptic nucleus. These fibers give rise to a plexus that occupies a significant portion of the subventricular zone, sometimes extending to the ventricle surface. Although the matter remains open to speculation, these fibers may be linked to the control of ciliary beating, as described by Conductier et al. (2013a, b). A cohort of these fibers ascend through the periventricular zone and are joined by fibers apparently originating from the ZI to innervate the midline thalamic nuclei. As periventricular fibers are also found in the subventricular zone of the lateral ventricles, it is possible that a plexus of MCH-ir fibers lines the whole ventricular system of the brain.

A distinctive cohort of immunoreactive fibers originating in the tuberal hypothalamus course through the median eminence (ME) to reach the posterior pituitary. Fibers are reliably found in the adjoining arcuate nucleus (Fig. $11 \mathrm{a}-\mathrm{a}^{\prime}$ ), especially on the lateral part of this nucleus, and then reach the ME, with a moderate number of fibers present in the internal lamina, while sparser fibers are found in the external lamina (Fig. 11b-b'). It is noteworthy that, in both cases, the fibers are highly varicose, suggesting a putative release of $\mathrm{MCH}$ directly into the $\mathrm{ME}$. As other works have demonstrated variations in $\mathrm{MCH}$ immunoreactivity in the ME linked to the reproductive status of females (Chiocchio et al. 2001; Gallardo et al. 2004), it is possible that $\mathrm{MCH}$ contributes to the modulation of medianosomes in this area. As varicose fibers are found in proximity to blood vessels in the external lamina, a release of $\mathrm{MCH}$ or NEI in the portal vasculature cannot be ruled out. A moderate number of fibers are found in the posterior pituitary, preferentially concentrated near the periphery of this gland, and some fibers can be observed in the intermediate lobe. As demonstrated by Cvetkovic et al. (2003), neurons in the $3 \mathrm{Vdc}$ and the perifornical 


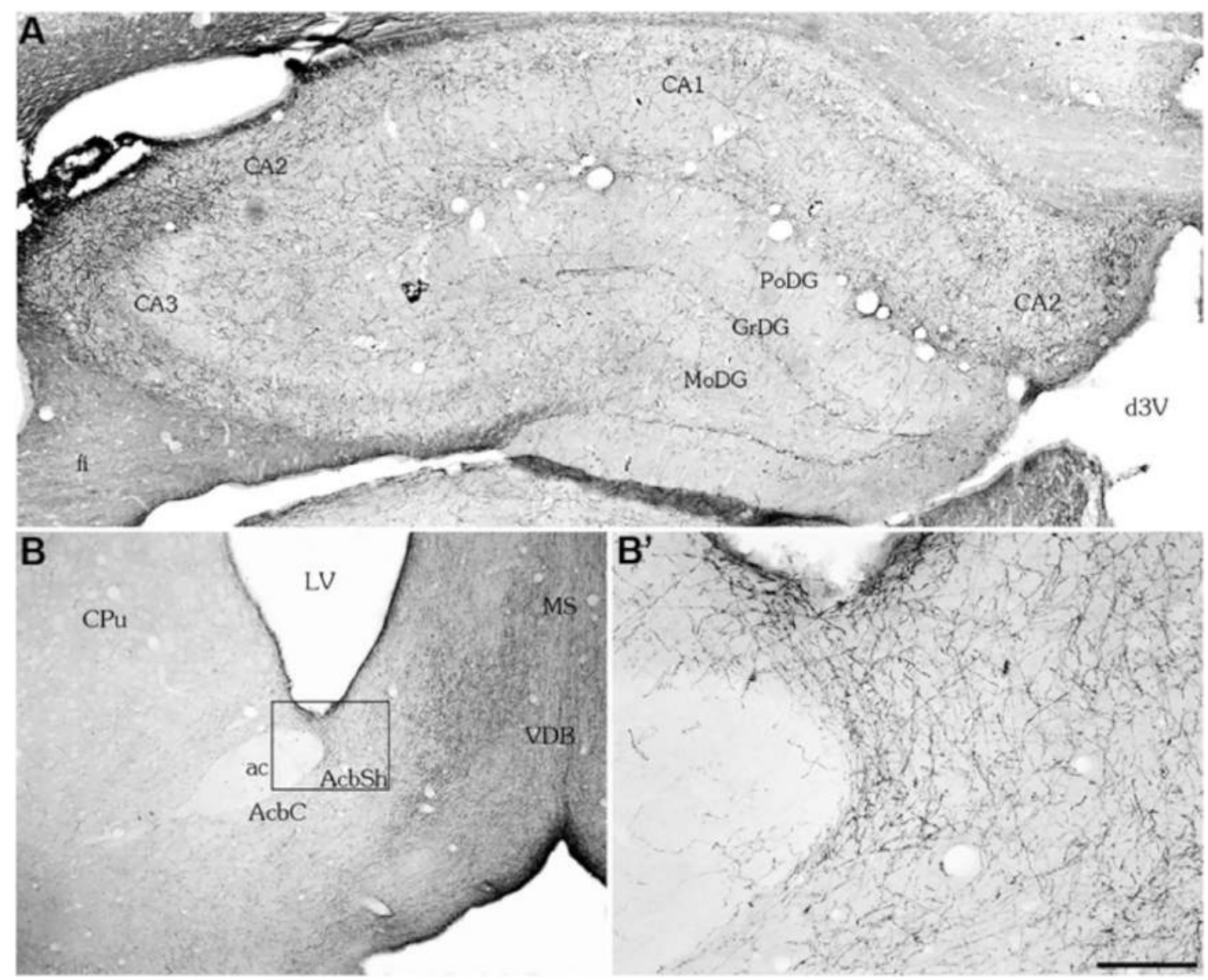

Fig. 10 The distribution of MCH-ir fibers in the rat neuroaxis, continued. Brightfield photomicrographs of frontal rat slices subjected to immunohistochemistry for $\mathrm{MCH}$ localization. (a) The hippocampal formation receives a high density of $\mathrm{MCH}$-ir fibers along most of its major subdivisions. Although the dentate gyrus (DG) is not innervated as densely as the hippocampus proper, $\mathrm{MCH}$-ir fibers are seen lining the subgranular zone. (b) The shell of the nucleus accumbens (AcbSh) contains immunoreactive fibers along most of its rostrocaudal extension. $\left(\mathbf{b}^{\prime}\right)$ Higher magnification of the rectangle in $\mathbf{b}$. Most MCH-ir fibers in the AcbSh are found in the area between the anterior commissure (ac) and the ventral tip of the lateral ventricle (LV). Other abbreviations: $A c b C$ core subdivision of the nucleus accumbens, CA1 cornu ammonis 1, CA2 cornu ammonis 2, CA3 cornu ammonis 3, CPu caudate nucleus and putamen, $G r D G$ granular layer of the dentate gyrus, $M o D G$ molecular layer of the dentate gyrus, $M S$ medial septal nucleus, $P o D G$ polymorphic layer of the dentate gyrus, $V D B$ vertical limb of the diagonal band of Broca. Scale bar: b, $500 \mu \mathrm{m}$; a, $200 \mu \mathrm{m}$; and $\mathbf{b}^{\prime}, 100 \mu \mathrm{m}$

nucleus are the main origins of the posterior pituitary projections, and some of these neurons project simultaneously to the cortex.

\subsection{Descending Pathway}

As is the case with the ascending pathway, the $m f b$ constitutes the main descent pathway of fibers to the caudal portion of the CNS. Secondary descending tracts 

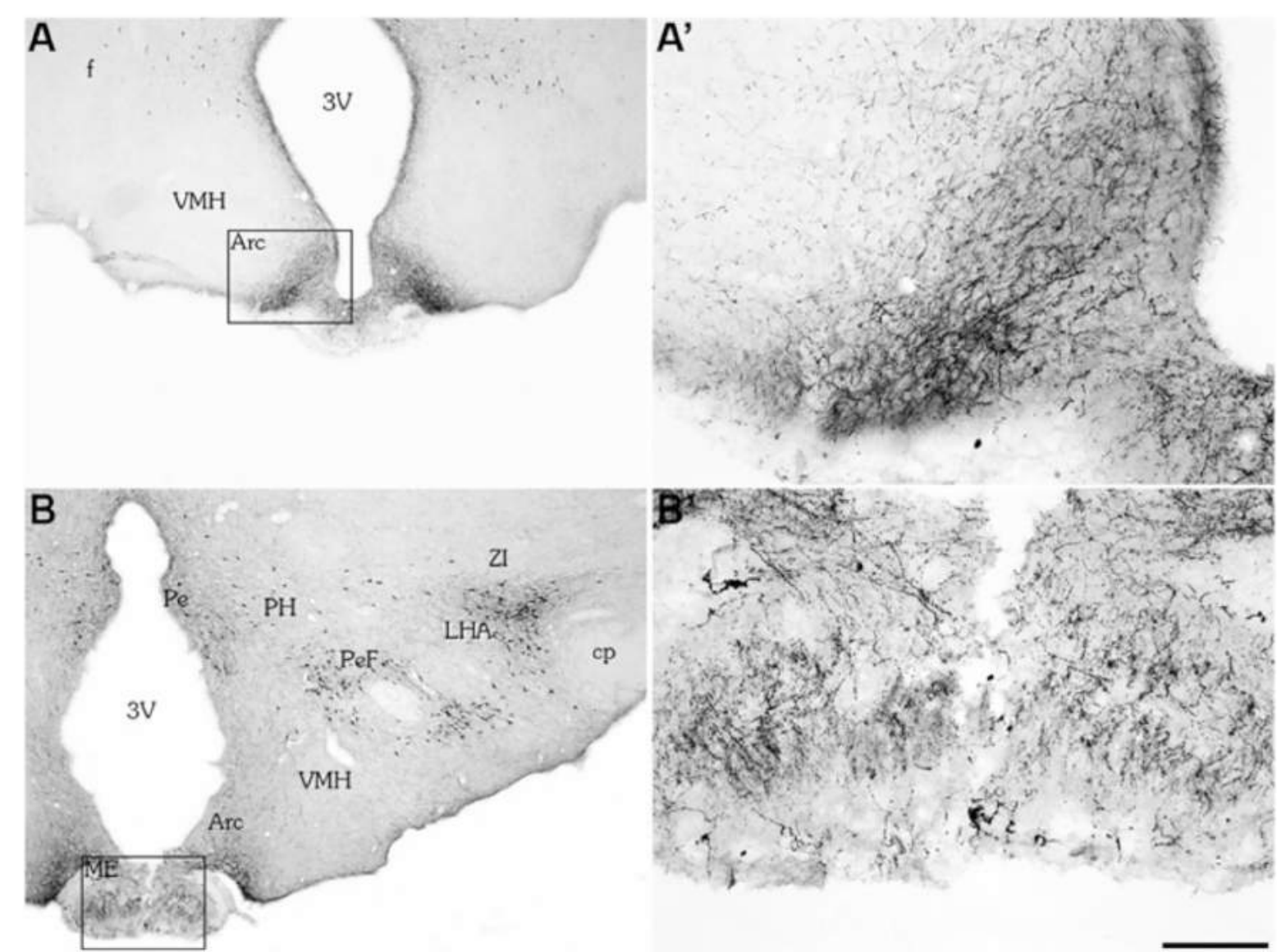

Fig. 11 The distribution of MCH-ir fibers in the rat neuroaxis, continued. Brightfield photomicrographs of frontal rat slices subjected to immunohistochemistry for $\mathrm{MCH}$ localization. (a) A great number of MCH-ir fibers are found in the lateral part of the arcuate nucleus (Arc). ( $\mathbf{a}^{\prime}$ ) Higher magnification of the rectangle in a. (b) Labeled fibers can be clearly seen in the median eminence $(\mathrm{EM})$ of the rat. $\left(\mathbf{b}^{\prime}\right)$ Higher magnification of the rectangle in $\mathbf{b}$. There is a preferential distribution of $\mathrm{MCH}$-ir fibers in the internal layer of the ME, although sparser fibers are found in the external layer. Other abbreviations: $3 \mathrm{~V}$ third ventricle, $c p$ cerebral peduncle, $f$ fornix, $L H A$ lateral hypothalamic area, $P e$ periventricular nucleus of the hypothalamus, $P e F$ perifornical nucleus, $P H$ posterior hypothalamic area, $V M H$ ventromedial hypothalamic nucleus; ZI zona incerta. Scale bar: a-b, $500 \mu \mathrm{m} ; \mathbf{a}^{\prime}-\mathbf{b}^{\prime}, 100 \mu \mathrm{m}$

include the fornix, mammillothalamic tract, and fasciculus retroflexus, which help supply the mammillary and interpeduncular nuclear complexes (Fig. 12a-c'). At the level of the diencephalic-mesencephalic transition, fibers in the descending pathway are identified as an array of fibers that occupy the space between the substantia nigra and the medial lemniscus. This pathway then forks at the level of the red nucleus, with a dorsomedial component innervating the central tegmental fields, the mesencephalic and pontine central gray and other nuclei of the reticular core (Fig. 13a-c'). The mesencephalic periaqueductal gray matter has a topological relationship with diencephalic $\mathrm{MCH}$ neurons, with dorsomedial and dorsolateral regions receiving fibers originating in the IHy, while the ventrolateral area receives fibers from the tuberal LHA (Elias and Bittencourt 1997). The ventral component will contribute to the innervation of the ventral tegmental area, the interpeduncular nuclear complex, the raphe nuclei, and the pontine gray. Along its course, nuclei implicated in food 

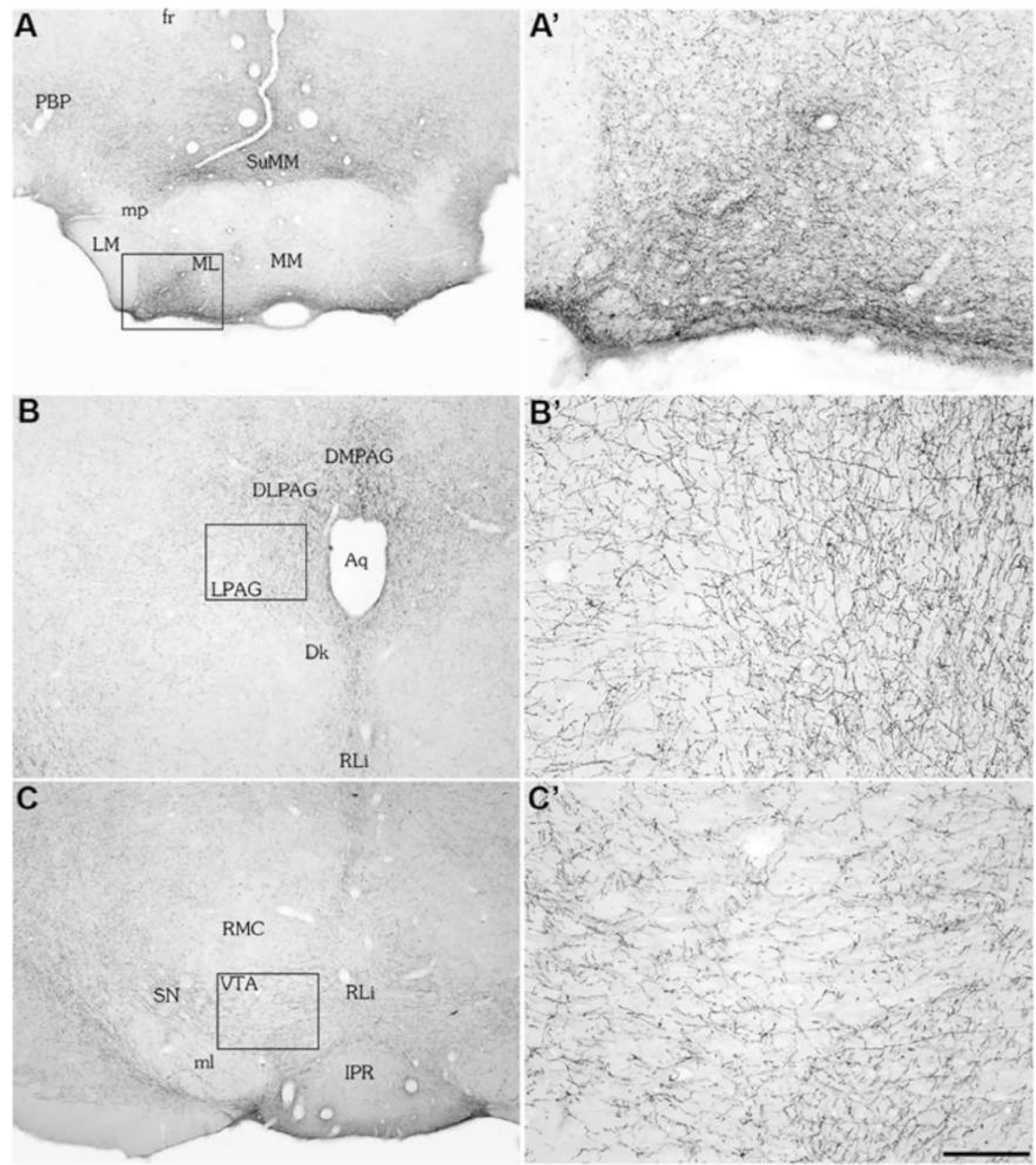

Fig. 12 The distribution of MCH-ir fibers in the rat neuroaxis, continued. Brightfield photomicrographs of frontal rat slices subjected to immunohistochemistry for MCH localization. (a) The lateral part of the medial mammillary nucleus (ML) has abundant innervation by $\mathrm{MCH}$-ir fibers, while only a moderate density reaches the adjoining medial part (MM), and there is a virtual absence of fibers in the lateral mammillary nucleus (LM). ( $\left.\mathbf{a}^{\prime}\right)$ Higher magnification of the rectangle in a highlighting the presence of fibers in the ML. (b) The periaqueductal gray matter also receives a high density of labeled fibers, including the very densely labeled dorsomedial (DMPAG) and dorsolateral (DLPAG) subdivisions. ( $\mathbf{b}^{\prime}$ ) Higher magnification of the rectangle in $\mathbf{b}$. The lateral PAG (LPAG) contains several radially oriented fibers. (c) Midbrain areas containing dopaminergic neurons receive inputs from $\mathrm{MCH}$ neurons, including the substantia nigra (SN) and the ventral tegmental area (VTA). ( $\mathbf{c}^{\prime}$ ) Higher magnification of the rectangle in $\mathbf{c}$, where several MCH-ir terminal fields can be seen in the VTA. Other abbreviations: Aq cerebral aqueduct, $D k$ nucleus of Darkschewitsch, fr fasciculus retroflexus, IPR interpeduncular nucleus, $m l$ medial lemniscus, $m p$ mammillary peduncle, $P B P$ parabrachial pigmented nucleus, $R L i$ rostral linear nucleus of raphe, $R M C$ magnocellular part of the red nucleus, SuMM medial part of the supramammillary nucleus. Scale bar: $\mathbf{a}-\mathbf{c}, 500 \mu \mathrm{m} ; \mathbf{a}^{\prime}-\mathbf{c}^{\prime}, 100 \mu \mathrm{m}$ 

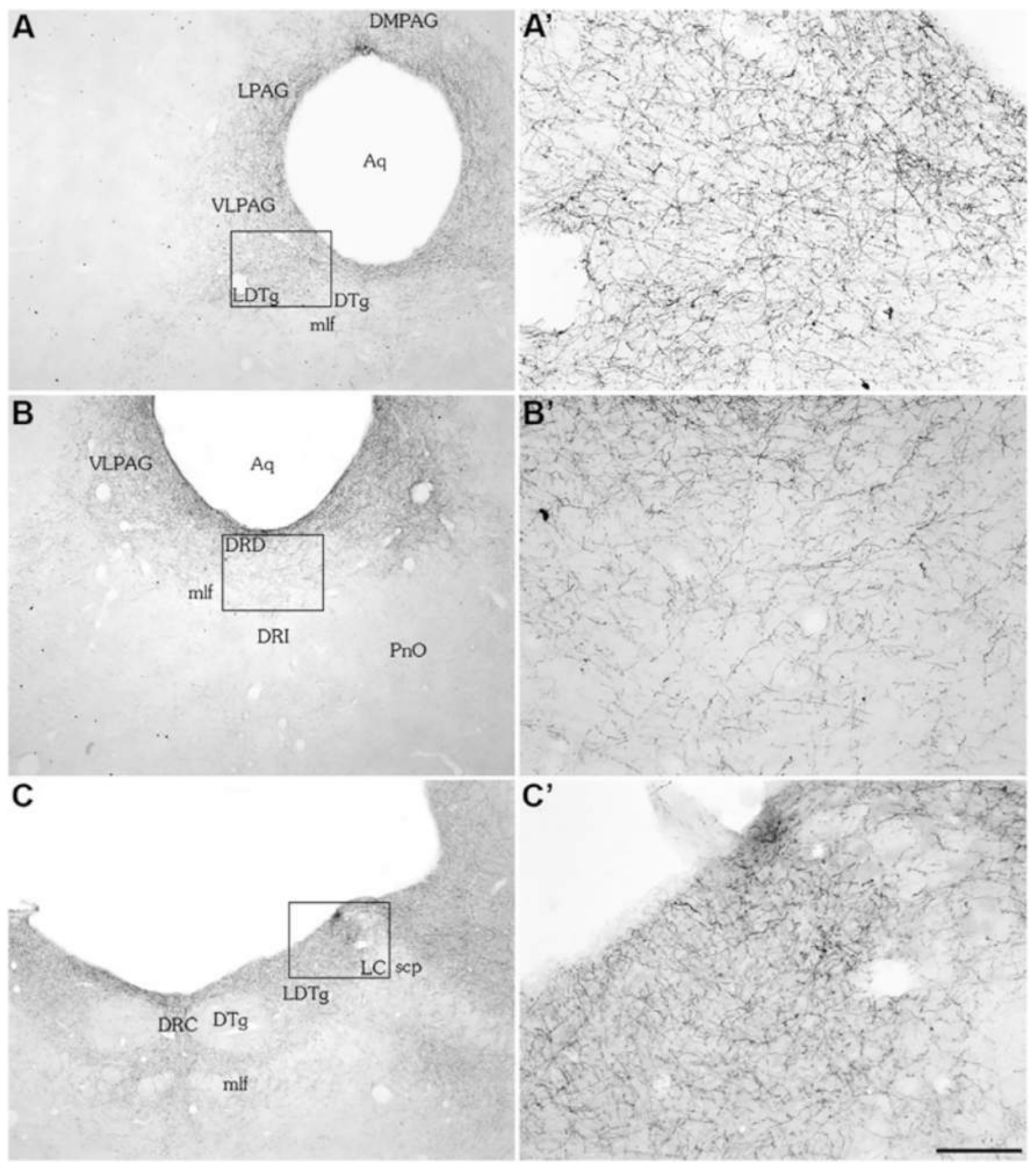

Fig. 13 The distribution of MCH-ir fibers in the rat neuroaxis, continued. Brightfield photomicrographs of frontal rat slices subjected to immunohistochemistry for MCH localization. (a) A high contingent of labeled fibers can be found encircling the cerebral aqueduct (Aq), including in the ventrolateral periaqueductal gray matter (VLPAG) and the laterodorsal tegmental nucleus (LDTg). $\left(\mathbf{a}^{\prime}\right)$ Higher magnification of the rectangle in $\mathbf{a}$, showcasing the numerous fibers en passant and terminal boutons found in the LDTg. (b) Fibers can also be found in the raphe nuclei, including the dorsal part of the dorsal raphe nucleus (DRD). ( $\left.\mathbf{b}^{\prime}\right)$ Higher magnification of the rectangle in $\mathbf{b}$. (c) The locus coeruleus (LC) has inputs originated from MCH neurons. $\left(\mathbf{c}^{\prime}\right)$ Higher magnification of the rectangle in c. Other abbreviations: $D M P A G$ dorsomedial part of the periaqueductal gray matter, $D R C$ central part of the dorsal raphe nucleus, $D R I$ interfascicular part of the dorsal raphe nucleus, $D T g$ dorsal tegmental area, $L P A G$ lateral periaqueductal gray matter, mlf medial longitudinal fasciculus, $\mathrm{PnO}$ oral part of the pontine reticular nucleus, scp superior cerebellar peduncle. Scale bar: $\mathbf{a}-\mathbf{c}, 500 \mu \mathrm{m} ; \mathbf{a}^{\prime}-\mathbf{c}^{\prime}, 100 \mu \mathrm{m}$ 
consumption, such as the superior salivary nucleus (salivary glands control) and the motor nucleus of the trigeminal nerve ( $\mathrm{V}$-masseter innervation) receive fibers from the descending pathway (Pérez et al. 2011). The pedunculopontine tegmental nucleus, an area implicated in motor and arousal function (Saper and Fuller 2017; Garcia-Rill et al. 1987; Rye 1997), also receives a significant input of MCH-ir fibers that originate broadly in GABAergic neurons in the IHy, LHA, and perifornical nucleus. Some of these neurons also project to the motor cortex, suggesting that $\mathrm{MCH}$ neurons participate in a cortico mesencephalic integration circuit (Elias et al. 2008). At more caudal levels, the descending pathway is a strip of fibers located at the medial edge of the trigeminal complex, further descending to all levels of the spinal cord through the dorsal funiculi.

\subsection{Local Hypothalamic Projections}

Virtually the whole rostrocaudal extent of the hypothalamus displays MCH-ir fibers to some degree. In all likelihood, these fibers result from a combination of fibers from the ascending, periventricular, pituitary, and descending pathways, in addition to local extensions of dendrites and axons from MCH-containing neurons. In the anterior hypothalamus, fibers are found in the median preoptic, anteroventral periventricular, ventrolateral preoptic (Fig. 14a-a'), medial preoptic, anterior hypothalamic, suprachiasmatic, supraoptic (Fig. 14b-b'), and paraventricular nuclei, in addition to the medial and lateral preoptic areas. In the tuberal hypothalamus, fibers can be found in the arcuate, dorsomedial, and tuberomammillary nuclei, as well as in the retrochiasmatic and lateral hypothalamic areas. Fibers in the ventromedial nucleus are sparse and often occupy the margins of this nucleus. In the posterior hypothalamus, fibers occupy the premammillary, supramammillary, and lateral and medial mammillary nuclei, with a large contingent of fibers found in the lateral part of the latter, as well as in the posterior hypothalamic area.

\section{Anatomical Distribution of MCH Receptor 1}

While the distribution of Pmch mRNA-expressing neurons has been reported for several mammals, anatomical mappings of $M c h r l$ distribution have been restricted to male rats (Hervieu et al. 2000; Saito et al. 2001; Kolakowski et al. 1996) and male mice (Chee et al. 2013), with brief descriptions available for the rhesus monkey, dog, and ferret (Tan et al. 2002). Several other groups provided additional information about additional areas and species through northern blotting, RNA dot blots, PCR, and limited in situ hybridization (Chambers et al. 1999; Saito et al. 1999; Hill et al. 2001; Mori et al. 2001; Sailer et al. 2001). In this section, we will briefly explore the areas described to contain Mchrl mRNA and highlight regions with conflicting information. 

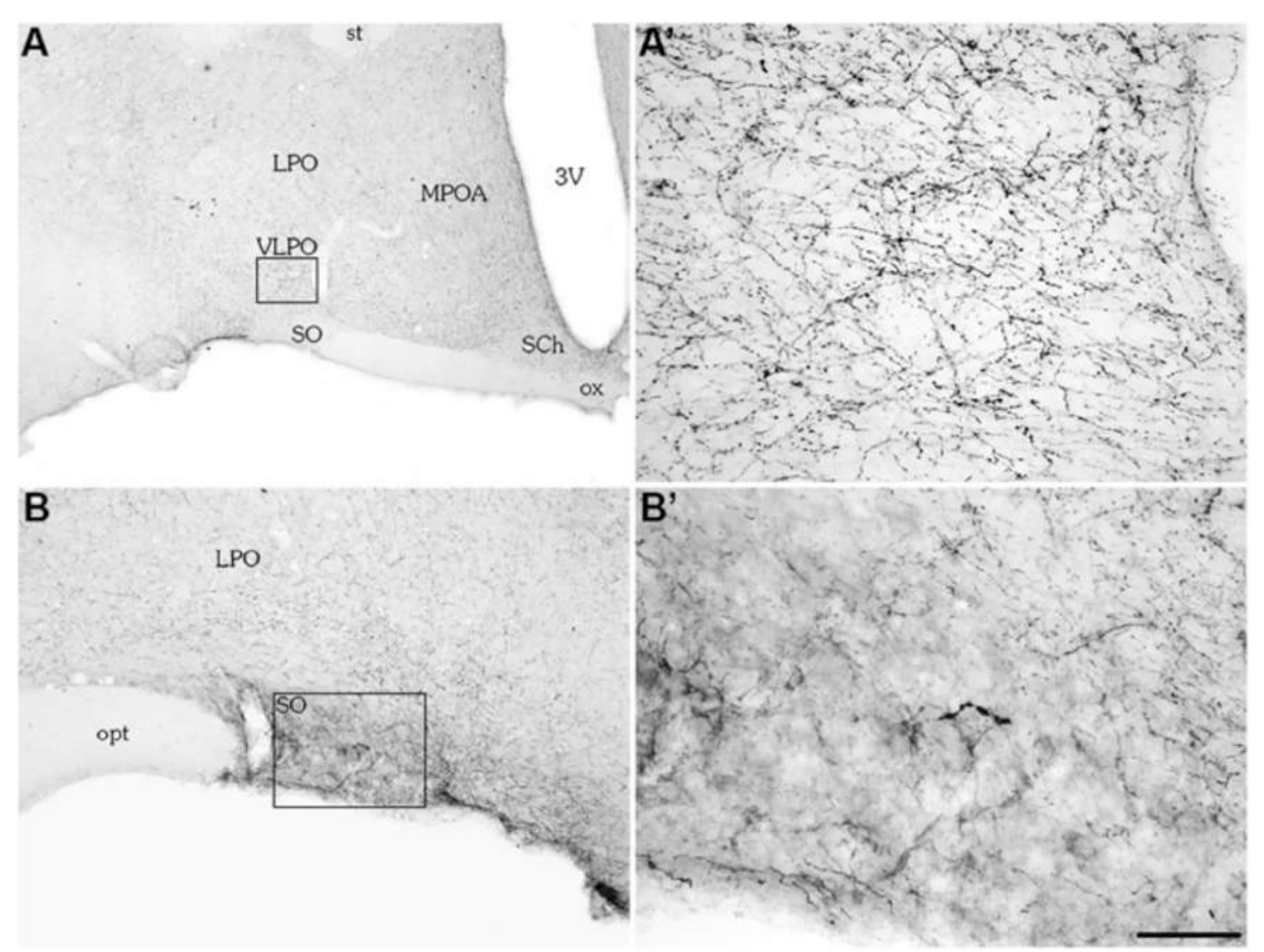

Fig. 14 The distribution of MCH-ir fibers in the rat neuroaxis, continued. Brightfield photomicrographs of frontal rat slices subjected to immunohistochemistry for MCH localization. (a) Numerous fibers and terminal boutons are found in the ventrolateral preoptic nucleus (VLPO). ( $\mathbf{a}^{\prime}$ ) Higher magnification of the rectangle in a. (b) Neuroendocrine nuclei, such as the supraoptic nucleus (SO), receive moderate $\mathrm{MCH}$ innervation. ( $\mathbf{b}^{\prime}$ ) Higher magnification of the rectangle in $\mathbf{b}$. Fibers in neuroendocrine nuclei are often enlarged and varicose. Other abbreviations: $3 \mathrm{~V}$ third ventricle, $L P O$ lateral preoptic area, MPOA medial preoptic area, opt optic tract, ox optic chiasm, SCh suprachiasmatic nucleus, st stria terminalis. Scale bar: $\mathbf{a}, 500 \mu \mathrm{m} ; \mathbf{b}, 200 \mu \mathrm{m}$; and $\mathbf{a}^{\prime}-\mathbf{b}^{\prime}, 50 \mu \mathrm{m}$

\subsection{Cortical Areas}

One of the most firmly established features of Mchrl mRNA is its high cortical expression. The distribution of Mchrl mRNA appears to be preferentially layerdependent, with the highest levels of expression located in layers II/III and V, although some have reported lower levels in layers IV and VI. Among the cortical fields that contain strong Mchrl mRNA signals are the frontal cortex; the primary and secondary motor areas; the primary and secondary somatosensory areas; the parietal, temporal, and orbital cortices; and the agranular and dysgranular insular cortices. Several sensory fields - such as the gustatory, visceral, and primary areas; the ventral and dorsal auditory areas; and the anterolateral, rostrolateral, posterolateral, and primary visual areas - contain receptors. Moderate expression is found in the cingulate cortex, the orbital cortex, and the entorhinal cortex. Low expression is 
detectable in the retrosplenial agranular and granular cortices. The expression of Mchrl mRNA in human cortical areas is supported by PCR data.

In addition to these cortical areas, there are consistent data pointing to high expression of Mchrl mRNA in olfactory-linked areas. Among the regions with high expression are the anterior olfactory nucleus, olfactory tubercle, piriform cortex, ventral and dorsal taenia tecta, indusium griseum, and nucleus of the lateral olfactory tract. Lower levels of expression were detected in the endopiriform nucleus. There are two major points of disagreement in the literature regarding olfactory regions. While Hervieu et al. (2000) described a high expression of Mchrl mRNA in the main olfactory bulb and the islands of Calleja, Saito et al. (2001) did not detect signals in these areas. The data of Kolakowski et al. (1996) support the presence of Mchrl mRNA in the major islands of Calleja, but further data are necessary to determine whether the $\mathrm{MCH}$ receptor is present in these regions. Owing to the diminished olfactory-dedicated areas in the primate brain, information is lacking on this regard.

\subsection{Telencephalic Subcortical Areas}

There is also ample support for Mchrl mRNA in telencephalic subcortical areas, although disagreements exist for some regions. According to Hervieu et al. (2000), the septal area has a high expression of Mchrl mRNA, with the highest expression found in the nucleus of the diagonal band of Broca and moderate expression found in the medial septal nucleus, vertical and horizontal limbs of the diagonal band, dorsal and ventral segments of the lateral septum, and bed nucleus of the stria terminalis (BNST). On the other hand, Saito et al. (2001) report a distribution that is almost complementary to that of Hervieu et al. (2000), with no labeling detected in the medial septal nucleus and very scarce labeling in the dorsal and ventral lateral septum but heavy labeling in the intermediate division of the lateral septum. However, multiple authors report moderate expression of Mchrl mRNA in the septohippocampal nucleus and subdivisions of the BNST. There is no additional information about the septal area, and so the presence of Mchrl mRNA in this area in rodents or primates remains debatable.

The hippocampus proper contains some of the highest levels of Mchrl mRNA in the whole CNS. According to multiple authors, elevated levels of mRNA are found in all CA subfields (CA1-CA3), especially in the pyramidal layer. Moderate to high expression is also reported for the subiculum. On the other hand, the expression of Mchrl mRNA in the dentate gyrus is disputable: while Kolakowski et al. (1996) and Hervieu et al. (2000) describe high expression levels in the granular layer of the dentate gyrus, Saito et al. (2001) report only a few scattered cells labeled in the granular and polymorphic layers. PCR data support the high expression of Mchrl mRNA in the human hippocampus proper.

There is also plentiful support for high expression of Mchrl mRNA in the amygdaloid complex. Hervieu et al. (2000) report labeling in the basolateral, 
basomedial, central, medial, cortical, lateral, and posterior amygdaloid nuclei. Saito et al. (2001) describe high expression levels in the posterolateral cortical nucleus, nucleus of the lateral olfactory tract, basolateral amygdala, and anterior amygdaloid nucleus. Moderate expression was found by these authors in the lateral division of the central nucleus; the lateral division of the anterior amygdaloid nucleus; the lateral, medial, and posteromedial cortical nuclei; and the amygdalohippocampal area. Lower levels were found in the anterior cortical and basomedial nuclei and the intercalated masses. Data obtained from PCR experiments report moderate to high concentrations of Mchrl mRNA in the human amygdaloid complex.

Finally, there are less coherent reports regarding mRNA expression in the basal nuclei and related structures. Hervieu et al. (2000) report moderate expression in the caudate-putamen nucleus, nucleus accumbens, and substantia innominata. No labeling was detected in the globus pallidus. Saito et al. (2001), on the other hand, describe no expression in the ventral pallidum, low expression in the caudateputamen, and high expression in the nucleus accumbens shell, while only scattered signals are found in the core subdivision. This dichotomy between core and shell in the nucleus accumbens is further supported by the results of Kolakowski et al. (1996), who also report low expression in the caudate-putamen. Expression in the claustrum is supported by multiple sources, but there is no consensus on the relative amount. In humans, moderate to low expression of Mchrl mRNA is supported in the nucleus accumbens, caudate-putamen, and globus pallidus.

\subsection{Thalamus, Subthalamus, and Epithalamus}

Hervieu et al. (2000) argue in favor of ample expression of Mchrl mRNA in the thalamus. Among the stained areas are the following: high expression (paraventricular, anterodorsal, reticular, ventral posterolateral, and ventral posteromedial thalamic nuclei), moderate (anteromedial, laterodorsal, reuniens, ventral anterior-lateral complex, ventral posterolateral, and parafascicular thalamic nuclei), and low (interanterodorsal, anteroventral, centromedial, paratenial, and centrolateral nuclei). In the subthalamic area, moderate staining is found in the subthalamic nucleus and strong labeling in the ZI. In the epithalamus, mRNA is detectable in the medial habenular nucleus but not in the lateral habenular nucleus. Finally, moderate labeling is found in the lateral geniculate nucleus. Saito et al. (2001) described a mostly similar distribution among nuclei but only low to moderate in intensity. Dense labeling in the zona incerta is confirmed by the latter authors, but no labeling was detected in the medial or lateral habenular nuclei. Data from PCR experiments indicate moderate to low levels of expression in the human thalamus. 


\subsection{Hypothalamus}

There are multiple reports of Mchrl mRNA expression in the hypothalamus. Most reports indicate expression in the ventromedial and dorsomedial hypothalamic nuclei for both rodents and a nonhuman primate. In addition to those two areas, Hervieu et al. (2000) also describe high expression in the paraventricular, periventricular, and supraoptic hypothalamic nuclei, in addition to the lateral and medial mammillary nuclei. Moderate or low expression was found in the median preoptic nucleus, medial preoptic area, anterior hypothalamic nucleus, arcuate nucleus, and lateral and posterior hypothalamic areas. Saito et al. (2001), on the other hand, describe high expression of Mchrl mRNA only in the lateral and medial mammillary nuclei. These authors also found scarce expression in the paraventricular and dorsomedial hypothalamic nuclei; the anterior, lateral, and posterior hypothalamic areas; and the premammillary and supramammillary nuclei. In contrast to the data from rodents, there is a scarcity of data on MCHR 1 in the human hypothalamus, with one report describing high expression and another describing low expression. No data are available about individual nuclei for humans.

\subsection{Midbrain, Hindbrain, and Spinal Cord}

Numerous structures in the reticular formation of the midbrain and hindbrain contain Mchrl mRNA expression. The most strongly supported structures are the substantia nigra and the ventral tegmental area, structures linked to the function of the basal nuclei. Hervieu et al. (2000) describe expression in several other structures, including the following: high (periaqueductal gray matter, interpeduncular nucleus, dorsal tegmental nucleus, olivary complex, and locus coeruleus) and moderate (anterior pretectal nucleus, raphe nuclei, red nucleus, olivary complex, and pontine reticular formation). Saito et al. (2001) found similar results, reporting high expression in the locus coeruleus and red nucleus, along with moderate expression in the pontine and gigantocellular reticular nuclei, dorsal raphe nucleus, and laterodorsal tegmental nucleus. Lower expression is reported by those authors in the parvicellular reticular nucleus, inferior olive, and medullary reticular field. In humans, there are reports of expression in the locus coeruleus, pons and medulla oblongata, but there is no consensus regarding the level of expression in the substantia nigra.

Several areas linked to sensory and visceral information processing were found to contain Mchrl mRNA expression, although reports are somewhat conflicting. Hervieu et al. (2000) report widespread distribution of mRNA, namely, the superficial and intermediate superior colliculus, inferior colliculus, anterior pretectal nucleus, olivary pretectal nucleus, principal sensory nucleus, dorsal and ventral cochlear nuclei, nucleus of the lateral lemniscus, rostral part of the nucleus of the solitary tract, parabrachial nucleus, and Kölliker-Fuse nucleus. Saito et al. (2001), on the other hand, found a very weak signal in the inferior colliculus. Regarding the 
motor system, labeled areas include the nucleus of the oculomotor nerve (III), spinal trigeminal nucleus, nucleus ambiguus, trigeminal motor nucleus, facial nucleus, hypoglossal nucleus, and dorsal motor nucleus of the vagus. The presence of Mchrl mRNA in the cerebellum, on the other hand, is highly disputable. While Hervieu et al. (2000) describe strong labeling in the granular layer of the cerebellar cortex and moderate labeling in the interpositus nucleus, Kolakowski et al. (1996) and Saito et al. (2001) found no evidence of expression in the cerebellum. In humans, moderate to low expression has been reported for the cerebellum.

Hervieu et al. (2000) detected Mchrl mRNA in the lumbar part of the spinal cord, including all subdivisions of the gray matter, with more pronounced staining in the dorsal horn. Data for humans suggest moderate to low expression of this receptor in the human spinal cord.

\section{Anatomical Distribution of MCH Receptor 2}

Owing to the phylogenetically restricted expression of MCHR2 mRNA, there are no data available for this receptor in rodents. Most of the data collected, therefore, come from human tissue that was probed by PCR and northern blotting. Additional in situ hybridization data are available for the ferret (Mustela putorius furo), dog (Canis lupus familiaris), rhesus (Macaca mulatta), and African green monkey (Chlorocebus sabaeus).

The consensus region of elevated MCHR2 mRNA expression is the cortex, with several works supporting gene expression in this structure (An et al. 2001; Hill et al. 2001; Mori et al. 2001; Sailer et al. 2001; Wang et al. 2001). Among the cortical regions with demonstrated expression are the frontal, temporal, parietal, and occipital lobes and the cingulate and paracentral gyri. Subcortical areas, such as the hippocampus, amygdaloid complex, caudate-putamen, globus pallidus, and nucleus accumbens, also express MCHR2 mRNA (An et al. 2001; Hill et al. 2001; Mori et al. 2001; Sailer et al. 2001; Wang et al. 2001; Rodriguez et al. 2001). Expression in other areas is more contentious. While An et al. (2001) and Sailer et al. (2001) report expression in the diencephalon, Hill et al. (2001), Mori et al. (2001), and Rodriguez et al. (2001) did not detect signals in this structure. The same applies to the midbrain and hindbrain, with An et al. (2001) detecting expression in the pons and medulla oblongata, Mori et al. (2001) labeling only the medulla oblongata, and Hill et al. (2001) not detecting expression in these structures. A similar situation is encountered for the substantia nigra, where Mori et al. (2001) detected labeling, but Hill et al. (2001) did not. The cerebellum and the spinal cord were consistently negative across studies (An et al. 2001; Hill et al. 2001; Mori et al. 2001; Rodriguez et al. 2001). Finally, it is uncertain whether MCHR2 mRNA expression occurs in fiber bundles, with An et al. (2001) and Mori et al. (2001) detecting labeling in the corpus callosum and cerebral peduncles, while Wang et al. (2001) describe a negative result for the corpus callosum. 
In the African green monkey, there is limited evidence of expression in the dorsomedial hypothalamus. On the other hand, expression is abundant in the anterior and lateral hypothalamic areas, as well as in the ventromedial nucleus (Sailer et al. 2001). In the rhesus monkey, in situ hybridization revealed signals in the medial and central amygdala, CA1 of the hippocampus, and entorhinal and temporal cortices, in addition to weaker signals in the hypothalamus, claustrum, and insular cortex. In the dog, MCHR2 mRNA was detected in the preoptic nuclei of the hypothalamus, the medial septum, and the claustrum. Finally, in the ferret, MCHR2 mRNA could be detected mainly in the dorsal claustrum (Tan et al. 2002).

\section{Conclusion}

From the initial discovery of $\mathrm{MCH}$ in the salmon pituitary to this day, an impressive amount of data has been collected about the MCH system. An amazing number of functions is associated with it, including modulation of the sleep-wake cycle; sensorimotor integration; ingestive, sexual, and maternal behaviors; energy expenditure; locomotor activity; autonomic modulation; thyroid modulation; reward; hormone secretion; foraging; memory; mood; ventricular homeostasis; and immune response, among others. All these functions are supported by a complex anatomical organization, including two peptides, a putative third peptidergic member of this family, alternative splicing and overprinted sequences, multiple loci with $\mathrm{MCH}$-synthesizing neurons, a vast array of projections, and two receptor paralogues that have distinct distributions in the CNS. All this complexity is underlined by a rich phylogenetic history in which, apparently, different levels of conservation pressure were applied to each member of this family. All these factors combine to result in a fascinating biological system that, despite everything that has been described about it, still cannot be considered completely understood.

Acknowledgments The writing of this chapter would not be possible without the funding granted by the Fundação de Amparo à Pesquisa do Estado de São Paulo [São Paulo Research Foundation-FAPESP], grants \#2010/52068-0 and \#2016/02224-1 [JCB] and \#2016/027480 [GBD]. JCB is also a recipient of a grant from the Coordenação de Aperfeiçoamento de Pessoal de Ensino Superior [Agency for the Advancement of Higher Education] and the Comite Français d'Evaluation de la Coopération Universitaire avec le Brésil [French Committee for the Evaluation of Academic and Scientific Cooperation with Brazil], CAPES-COFECUB grant \#848/ 15. JCB is an investigator with the Conselho Nacional de Desenvolvimento Científico e Tecnológico [National Council for Scientific and Technological Development-CNPq, Brazil], recipient of grant \#426378/2016-4. 


\section{References}

Alberto CO, Trask RB, Hirasawa M (2011) Dopamine acts as a partial agonist for $\alpha 2 \mathrm{~A}$ adrenoceptor in melanin-concentrating hormone neurons. J Neurosci 31(29):10671-10676. https://doi.org/10. 1523/JNEUROSCI.6245-10.2011

Allaeys I, Bouyer K, Loudes C, Faivre-Bauman A, Petit F, Ortola C, Cardinaud B, Epelbaum J, Nahon JL (2004) Characterization of MCH-gene-overprinted-polypeptide-immunoreactive material in hypothalamus reveals an inhibitory role of pro-somatostatin1-64 on somatostatin secretion. Eur J Neurosci 19(4):925-936. https://doi.org/10.1111/j.0953-816X.2004.03187.x

Alvisi R, Diniz G, Da-Silva J, Bittencourt J, Felicio L (2016) Suckling-induced Fos activation and melanin-concentrating hormone immunoreactivity during late lactation. Life Sci 148:241-246. https://doi.org/10.1016/j.lfs.2016.02.038

An S, Cutler G, Zhao JJ, Huang SG, Tian H, Li W, Liang L, Rich M, Bakleh A, Du J, Chen JL, Dai $\mathrm{K}$ (2001) Identification and characterization of a melanin-concentrating hormone receptor. Proc Natl Acad Sci USA 98(13):7576-7581. https://doi.org/10.1073/pnas. 131200698

Bachner D, Kreienkamp H, Weise C, Buck F, Richter D (1999) Identification of melanin concentrating hormone $(\mathrm{MCH})$ as the natural ligand for the orphan somatostatin-like receptor 1 (SLC-1). FEBS Lett 457(3):522-524. https://doi.org/10.1016/S0014-5793(99)01092-3

Bäckberg M, Ultenius C, Fritschy JM, Meister B (2004) Cellular localization of GABAA receptor $\alpha$ subunit immunoreactivity in the rat hypothalamus: relationship with neurones containing orexigenic or anorexigenic peptides. J Neuroendocrinol 16(7):589-604. https://doi.org/10. $1111 /$ j. 1365-2826.2004.01207.x

Banisadr G, Gosselin RD, Mechighel P, Kitabgi P, Rostène W, Parsadaniantz SM (2005) Highly regionalized neuronal expression of monocyte chemoattractant protein-1 (MCP-1/CCL2) in rat brain: evidence for its colocalization with neurotransmitters and neuropeptides. J Comp Neurol 489(3):275-292. https://doi.org/10.1002/cne.20598

Bittencourt JC (2011) Anatomical organization of the melanin-concentrating hormone peptide family in the mammalian brain. Gen Comp Endocrinol 172(2):185-197. https://doi.org/10. 1016/j.ygcen.2011.03.028

Bittencourt J, Celis ME (2008) Anatomy, function and regulation of neuropeptide EI (NEI). Peptides 29(8):1441-1450. https://doi.org/10.1016/j.peptides.2008.03.012

Bittencourt JC, Presse F, Arias C, Peto C, Vaughan J, Nahon JL, Vale W, Sawchenko PE (1992) The melanin-concentrating hormone system of the rat brain: an immuno- and hybridization histochemical characterization. J Comp Neurol 319(2):218-245. https://doi.org/10.1002/cne. 903190204

Bleier R, Cohn P, Siggelkow IR (1979) A cytoarchitectonic atlas of the hypothalamus and hypothalamic third ventricle of the rat. In: Morgane PJ, Panksepp J (eds) Anatomy of the hypothalamus, vol l. Marcel Dekker, New York, pp 137-220

Borsu L, Presse F, Nahon JL (2000) The AROM gene, spliced mRNAs encoding new DNA/RNAbinding proteins are transcribed from the opposite strand of the melanin-concentrating hormone gene in mammals. J Biol Chem 275(51):40576-40587. https://doi.org/10.1074/jbc. M006524200

Brailoiu GC, Dun SL, Brailoiu E, Inan S, Yang J, Chang JK, Dun NJ (2007) Nesfatin-1: distribution and interaction with a $\mathrm{G}$ protein-coupled receptor in the rat brain. Endocrinology 148 (10):5088-5094. https://doi.org/10.1210/en.2007-0701

Breton C, Presse F, Hervieu G, Nahon JL (1993a) Structure and regulation of the mouse melaninconcentrating hormone mRNA and gene. Mol Cell Neurosci 4(3):271-284. https://doi.org/10. 1006/mone.1993.1035

Breton C, Schorpp M, Nahon JL (1993b) Isolation and characterization of the human melaninconcentrating hormone gene and a variant gene. Brain Res Mol Brain Res 18(4):297-310. https://doi.org/10.1016/0169-328X(93)90093-5 
Neuroanatomical Structure of the MCH System

Burdakov D, Gerasimenko O, Verkhratsky A (2005) Physiological changes in glucose differentially modulate the excitability of hypothalamic melanin-concentrating hormone and orexin neurons in situ. J Neurosci 25(9):2429-2433. https://doi.org/10.1523/JNEUROSCI.4925-04.2005

Burgaud JL, Poosti R, Fehrentz JA, Martinez J, Nahon JL (1997) Melanin-concentrating hormone binding sites in human SVK14 keratinocytes. Biochem Biophys Res Commun 241(3):622-629. https://doi.org/10.1006/bbrc. 1997.7849

Casatti CA, Elias CF, Sita LV, Frigo L, Furlani VC, Bauer JA, Bittencourt JC (2002) Distribution of melaninconcentrating hormone neurons projecting to the medial mammillary nucleus. Neuroscience 115(3):899-915. https://doi.org/10.1016/S0306-4522(02)00508-0

Chambers J, Ames RS, Bergsma D, Muir A, Fitzgerald LR, Hervieu G, Dytko GM, Foley JJ, Martin J, Liu WS, Park J, Ellis C, Ganguly S, Konchar S, Cluderay J, Leslie R, Wilson S, Sarau HM (1999) Melanin-concentrating hormone is the cognate ligand for the orphan G-proteincoupled receptor SLC-1. Nature 400(6741):261-265. https://doi.org/10.1038/22313

Chee MJ, Pissios P, Maratos-Flier E (2013) Neurochemical characterization of neurons expressing melanin-concentrating hormone receptor 1 in the mouse hypothalamus. J Comp Neurol 521 (10):2208-2234. https://doi.org/10.1002/cne.23273

Chee MJ, Arrigoni E, Maratos-Flier E (2015) Melanin-concentrating hormone neurons release glutamate for feedforward inhibition of the lateral septum. J Neurosci 35(8):3644-3651. https://doi.org/ 10.1523/JNEUROSCI.4187-14.2015

Chiocchio SR, Gallardo MG, Louzan P, Gutnisky V, Tramezzani JH (2001) Melanin-concentrating hormone stimulates the release of luteinizing hormone-releasing hormone and gonadotropins in the female rat acting at both median eminence and pituitary levels. Biol Reprod 64 (5):1466-1472. https://doi.org/10.1095/biolreprod64.5.1466

Chou TC, Rotman SR, Saper CB (2004) Lateral hypothalamic acetylcholinesteraseimmunoreactive neurons co-express either orexin or melanin concentrating hormone. Neurosci Lett 370(2-3):123-126. https://doi.org/10.1016/j.neulet.2004.08.070

Collin M, Bäckberg M, Önnestam K, Meister B (2002) 5-HT1A receptor immunoreactivity in hypothalamic neurons involved in body weight control. Neuroreport 13(7):945-951. https://doi. org/10.1097/00001756-200205240-00009

Conductier G, Nahon J-L, Guyon A (2011) Dopamine depresses melanin concentrating hormone neuronal activity through multiple effects on $\alpha 2$-noradrenergic, D1 and D2-like dopaminergic receptors. Neuroscience 178:89-100. https://doi.org/10.1016/j.neuroscience.2011.01.030

Conductier G, Brau F, Viola A, Langlet F, Ramkumar N, Dehouck B, Lemaire T, Chapot R, Lucas L, Rovère C (2013a) Melanin-concentrating hormone regulates beat frequency of ependymal cilia and ventricular volume. Nat Neurosci 16(7):845-847. https://doi.org/10. 1038/nn.3401

Conductier G, Martin A, Risold P-Y, Jego S, Lavoie R, Lafont C, Mollard P, Adamantidis A, Nahon J-L (2013b) Control of ventricular ciliary beating by the melanin concentrating hormoneexpressing neurons of the lateral hypothalamus: a functional imaging survey. Front Endocrinol 4:182. https://doi.org/10.3389/fendo.2013.00182

Courseaux A, Nahon J-L (2001) Birth of two chimeric genes in the Hominidae lineage. Science 291 (5507):1293-1297. https://doi.org/10.1126/science.1057284

Cvetkovic V, Brischoux F, Griffond B, Bernard G, Jacquemard C, Fellmann D, Risold PY (2003) Evidence of melanin-concentrating hormone-containing neurons supplying both cortical and neuroendocrine projections. Neuroscience 116(1):31-35. https://doi.org/10.1016/S0306-4522 (02)00557-2

de Lecea L, Kilduff TS, Peyron C, Gao X, Foye PE, Danielson PE, Fukuhara C, Battenberg EL, Gautvik VT, Bartlett FS 2nd, Frankel WN, van den Pol AN, Bloom FE, Gautvik KM, Sutcliffe JG (1998) The hypocretins: hypothalamus-specific peptides with neuroexcitatory activity. Proc Natl Acad Sci USA 95(1):322-327

Diniz GB, Bittencourt JC (2017) The melanin-concentrating hormone as an integrative peptide driving motivated behaviors. Front Syst Neurosci 11:32. https://doi.org/10.3389/fnsys.2017.00032 
Douglass J, McKinzie AA, Couceyro P (1995) PCR differential display identifies a rat brain mRNA that is transcriptionally regulated by cocaine and amphetamine. J Neurosci 15(3):2471-2481

Drozdz R, Eberle AN (1995) Binding sites for melanin-concentrating hormone (MCH) in brain synaptosomes and membranes from peripheral tissues identified with highly tritiated $\mathrm{MCH}$. J Recept Signal Transduct Res 15(1-4):487-502. https://doi.org/10.3109/10799899509045235

Drozdz R, Baker BI, Zeller A, Eberle AN (1993) Synthesis and biological activity of highly tritiated rat/human melanin-concentrating hormone. Ann N Y Acad Sci 680(1):489-492. https://doi.org/ 10.1016/0014-5793(95)00043-9

Drozdz R, Siegrist W, Baker BI, Chluba-de Tapia J, Eberle AN (1995) Melanin-concentrating hormone binding to mouse melanoma cells in vitro. FEBS Lett 359(2-3):199-202. https://doi. org/10.1016/0014-5793(95)00043-9

Elias CF, Bittencourt JC (1997) Study of the origins of melanin-concentrating hormone and neuropeptide EI immunoreactive projections to the periaqueductal gray matter. Brain Res 755 (2):255-271. https://doi.org/10.1016/S0006-8993(97)00104-2

Elias CF, Saper CB, Maratos-Flier E, Tritos NA, Lee C, Kelly J, Tatro JB, Hoffman GE, Ollmann MM, Barsh GS, Sakurai T, Yanagisawa M, Elmquist JK (1998) Chemically defined projections linking the mediobasal hypothalamus and the lateral hypothalamic area. J Comp Neurol 402 (4):442-459. https://doi.org/10.1016/S0006-8993(98)00598-8

Elias CF, Lee CE, Kelly JF, Ahima RS, Kuhar M, Saper CB, Elmquist JK (2001) Characterization of CART neurons in the rat and human hypothalamus. J Comp Neurol 432(1):1-19. https://doi. org/10.1002/cne. 1085

Elias CF, Sita LV, Zambon BK, Oliveira ER, Vasconcelos LA, Bittencourt JC (2008) Melaninconcentrating hormone projections to areas involved in somatomotor responses. J Chem Neuroanat 35(2):188-201. https://doi.org/10.1016/j.jchemneu.2007.10.002

Ferreira JGP, Bittencourt JC, Adamantidis A (2017a) Melanin-concentrating hormone and sleep. Curr Opin Neurobiol 44:152-158. https://doi.org/10.1016/j.conb.2017.04.008

Ferreira JGP, Duarte JCG, Diniz GB, Bittencourt JC (2017b) Litter size determines the number of melanin-concentrating hormone neurons in the medial preoptic area of Sprague Dawley lactating dams. Physiol Behav 181:75-79. https://doi.org/10.1016/j.physbeh.2017.08.028

Foo KS, Brismar H, Broberger C (2008) Distribution and neuropeptide coexistence of nucleobindin-2 mRNA/nesfatin-like immunoreactivity in the rat CNS. Neuroscience 156 (3):563-579. https://doi.org/10.1016/j.neuroscience.2008.07.054

Fort P, Salvert D, Hanriot L, Jego S, Shimizu H, Hashimoto K, Mori M, Luppi P-H (2008) The satiety molecule nesfatin-1 is co-expressed with melanin concentrating hormone in tuberal hypothalamic neurons of the rat. Neuroscience 155(1):174-181. https://doi.org/10.1016/j.neuro science.2008.05.035

Gallardo MG, Chiocchio SR, Tramezzani JH (2004) Changes of melanin-concentrating hormone related to LHRH release in the median eminence of rats. Brain Res 1030(1):152-158. https://doi.org/10. 1016/j.brainres.2004.10.005

Garcia SV, Libourel P-A, Lazarus M, Grassi D, Luppi P-H, Fort P (2017) Genetic inactivation of glutamate neurons in the rat sublaterodorsal tegmental nucleus recapitulates REM sleep behaviour disorder. Brain 140(2):414-428. https://doi.org/10.1093/brain/aww310

Garcia-Rill E, Houser C, Skinner R, Smith W, Woodward D (1987) Locomotion-inducing sites in the vicinity of the pedunculopontine nucleus. Brain Res Bull 18(6):731-738. https://doi.org/10. 1016/0361-9230(87)90208-5

Griffond B, Ciofi P, Bayer L, Jacquemard C, Fellmann D (1997) Immunocytochemical detection of the neurokinin $\mathrm{B}$ receptor (NK3) on melanin-concentrating hormone $(\mathrm{MCH})$ neurons in rat brain. J Chem Neuroanat 12(3):183-189. https://doi.org/10.1016/S0891-0618(96)00200-1

Haemmerle CA, Campos AM, Bittencourt JC (2015) Melanin-concentrating hormone inputs to the nucleus accumbens originate from distinct hypothalamic sources and are apposed to GABAergic and cholinergic cells in the Long-Evans rat brain. Neuroscience 289:392-405. https://doi.org/10. 1016/j.neuroscience.2015.01.014 
Hausen AC, Ruud J, Jiang H, Hess S, Varbanov H, Kloppenburg P, Brüning JC (2016) Insulindependent activation of $\mathrm{MCH}$ neurons impairs locomotor activity and insulin sensitivity in obesity. Cell Rep 17(10):2512-2521. https://doi.org/10.1016/j.celrep.2016.11.030

Hazai D, Lien C-F, Hajós F, Halasy K, Górecki DC, Jancsik V (2008) Synaptic alpha-dystrobrevin: localization of a short alpha-dystrobrevin isoform in melanin-concentrating hormone neurons of the hypothalamus. Brain Res 1201:52-59. https://doi.org/10.1016/j.brainres.2008.01.046

Hervieu GJ, Cluderay JE, Harrison D, Meakin J, Maycox P, Nasir S, Leslie RA (2000) The distribution of the mRNA and protein products of the melanin-concentrating hormone $(\mathrm{MCH})$ receptor gene, slc-1, in the central nervous system of the rat. Eur J Neurosci 12(4):1194-1216. https://doi.org/10.1046/j.1460-9568.2000.00008.x

Hill J, Duckworth M, Murdock P, Rennie G, Sabido-David C, Ames RS, Szekeres P, Wilson S, Bergsma DJ, Gloger IS, Levy DS, Chambers JK, Muir AI (2001) Molecular cloning and functional characterization of $\mathrm{MCH} 2$, a novel human $\mathrm{MCH}$ receptor. J Biol Chem 276 (23):20125-20129. https://doi.org/10.1074/jbc.M102068200

Hintermann E, Tanner H, Talke-Messerer C, Schlumberger S, Zumsteg U, Eberle AN (2001) Interaction of melanin-concentrating hormone $(\mathrm{MCH})$, neuropeptide EI (NEI), neuropeptide GE (NGE), and $\alpha$-MSH with melanocortin and MCH receptors on mouse B 16 melanoma cells. J Recept Signal Transduct Res 21(1):93-116. https://doi.org/10.1081/RRS-100107145

Hotta K, Hosaka M, Tanabe A, Takeuchi T (2009) Secretogranin II binds to secretogranin III and forms secretory granules with orexin, neuropeptide Y, and POMC. J Endocrinol 202(1):111-121. https://doi.org/10.1677/JOE-08-0531

Huang H, van den Pol AN (2007) Rapid direct excitation and long-lasting enhancement of NMDA response by group I metabotropic glutamate receptor activation of hypothalamic melaninconcentrating hormone neurons. J Neurosci 27(43):11560-11572. https://doi.org/10.1523/ JNEUROSCI.2147-07.2007

Kampe J, Tschop MH, Hollis JH, Oldfield BJ (2009) An anatomic basis for the communication of hypothalamic, cortical and mesolimbic circuitry in the regulation of energy balance. Eur J Neurosci 30(3):415-430. https://doi.org/10.1111/j.1460-9568.2009.06818.x

Kawauchi H, Kawazoe I, Tsubokawa M, Kishida M, Baker BI (1983) Characterization of melaninconcentrating hormone in chum salmon pituitaries. Nature 305(5932):321-323. https://doi.org/ $10.1038 / 305321 \mathrm{a} 0$

Knollema S, Brown ER, Vale W, Sawchenko PE (1992) Novel hypothalamic and preoptic sites of prepro-melanin-concentrating hormone messenger ribonucleic acid and peptide expression in lactating rats. J Neuroendocrinol 4(6):709-717. https://doi.org/10.1111/j.1365-2826.1992.tb00222.x

Kolakowski LF Jr, Jung BP, Nguyen T, Johnson MP, Lynch KR, Cheng R, Heng HH, George SR, O'Dowd BF (1996) Characterization of a human gene related to genes encoding somatostatin receptors. FEBS Lett 398(2-3):253-258. https://doi.org/10.1016/S0014-5793(96)01160-X

Kong D, Vong L, Parton LE, Ye C, Tong Q, Hu X, Choi B, Brüning JC, Lowell BB (2010) Glucose stimulation of hypothalamic MCH neurons involves K ATP channels, is modulated by UCP2, and regulates peripheral glucose homeostasis. Cell Metab 12(5):545-552. https://doi.org/10. 1016/j.cmet.2010.09.013

Krolewski DM, Medina A, Kerman IA, Bernard R, Burke S, Thompson RC, Bunney WE, Schatzberg AF, Myers RM, Akil H (2010) Expression patterns of corticotropin-releasing factor, arginine vasopressin, histidine decarboxylase, melanin-concentrating hormone, and orexin genes in the human hypothalamus. J Comp Neurol 518(22):4591-4611. https://doi.org/10. $1002 /$ cne.22480

Lakaye B, Minet A, Zorzi W, Grisar T (1998) Cloning of the rat brain cDNA encoding for the SLC-1 G protein-coupled receptor reveals the presence of an intron in the gene. Biochim Biophys Acta 1401(2):216-220. https://doi.org/10.1016/S0167-4889(97)00135-3

Lembo PM, Grazzini E, Cao J, Hubatsch DA, Pelletier M, Hoffert C, St-Onge S, Pou C, Labrecque J, Groblewski T, O’Donnell D, Payza K, Ahmad S, Walker P (1999) The receptor for the orexigenic peptide melanin-concentrating hormone is a G-protein-coupled receptor. Nat Cell Biol 1(5):267-271. https://doi.org/10.1038/12978

Lima FF, Sita LV, Oliveira AR, Costa HC, da Silva JM, Mortara RA, Haemmerle CA, Xavier GF, Canteras NS, Bittencourt JC (2013) Hypothalamic melanin-concentrating hormone projections 
to the septo-hippocampal complex in the rat. J Chem Neuroanat 47:1-14. https://doi.org/10. 1016/j.jchemneu.2012.10.003

Macdonald D, Murgolo N, Zhang R, Durkin JP, Yao X, Strader CD, Graziano MP (2000) Molecular characterization of the melanin-concentrating hormone/receptor complex: identification of critical residues involved in binding and activation. Mol Pharmacol 58(1):217-225. https://doi.org/10.1124/mol.58.1.217

Mickelsen LE, Kolling FW, Chimileski BR, Fujita A, Norris C, Chen K, Nelson CE, Jackson AC (2017) Neurochemical heterogeneity among lateral hypothalamic hypocretin/orexin and melanin-concentrating hormone neurons identified through single-cell gene expression analysis. eNeuro 4(5). https://doi.org/10.1523/ENEURO.0013-17.2017

Modirrousta M, Mainville L, Jones BE (2005) Orexin and MCH neurons express c-Fos differently after sleep deprivation vs. recovery and bear different adrenergic receptors. Eur J Neurosci 21 (10):2807-2816. https://doi.org/10.1111/j.1460-9568.2005.04104.x

Moldovan G-L, Dejsuphong D, Petalcorin MI, Hofmann K, Takeda S, Boulton SJ, D’Andrea AD (2012) Inhibition of homologous recombination by the PCNA-interacting protein PARI. Mol Cell 45(1):75-86. https://doi.org/10.1016/j.molcel.2011.11.010

Mori M, Harada M, Terao Y, Sugo T, Watanabe T, Shimomura Y, Abe M, Shintani Y, Onda H, Nishimura O, Fujino M (2001) Cloning of a novel G protein-coupled receptor, SLT, a subtype of the melanin-concentrating hormone receptor. Biochem Biophys Res Commun 283 (5):1013-1018. https://doi.org/10.1006/bbrc.2001.4893

Nahon JL, Presse F, Bittencourt JC, Sawchenko PE, Vale W (1989) The rat melanin-concentrating hormone messenger ribonucleic acid encodes multiple putative neuropeptides coexpressed in the dorsolateral hypothalamus. Endocrinology 125(4):2056-2065. https://doi.org/10.1210/ endo-125-4-2056

Nahon J-L, Joly C, Levan G, Szpirer J, Szpirer C (1992) Pro-melanin-concentrating hormone gene $(\mathrm{PMCH})$ is localized on human chromosome $12 \mathrm{q}$ and rat chromosome 7. Genomics 12 (4):846-848. https://doi.org/10.1016/0888-7543(92)90323-K

Nahon JL, Presse F, Breton C, Hervieu G, Schorpp M (1993) Structure and regulation of the melanin-concentrating hormone gene. Ann N Y Acad Sci 680:111-129. https://doi.org/10. $1111 /$ j.1749-6632.1993.tb19678.x

Nieuwenhuys R, Geeraedts LM, Veening JG (1982) The medial forebrain bundle of the rat. I. General introduction. J Comp Neurol 206(1):49-81. https://doi.org/10.1002/cne.902060106

Parkes D, Vale W (1992) Secretion of melanin-concentrating hormone and neuropeptide-EI from cultured rat hypothalamic cells. Endocrinology 131(4):1826-1831. https://doi.org/10.1210/ endo.131.4.1327720

Parks GS, Olivas ND, Ikrar T, Sanathara NM, Wang L, Wang Z, Civelli O, Xu X (2014a) Histamine inhibits the melanin-concentrating hormone system: implications for sleep and arousal. J Physiol 592(10):2183-2196. https://doi.org/10.1113/jphysiol.2013.268771

Parks GS, Wang L, Wang Z, Civelli O (2014b) Identification of neuropeptide receptors expressed by melanin-concentrating hormone neurons. J Comp Neurol 522(17):3817-3833. https://doi. org/10.1002/cne.23642

Parsons MP, Hirasawa M (2011) GIRK channel-mediated inhibition of melanin-concentrating hormone neurons by nociceptin/orphanin FQ. J Neurophysiol 105(3):1179-1184. https://doi. org/10.1152/jn.00791.2010

Pedeutour F, Szpirer C, Nahon JL (1994) Assignment of the human pro-melanin-concentrating hormone gene $(\mathrm{PMCH})$ to chromosome 12q23-q24 and two variant genes (PMCH1 and PMCHL2) to chromosome 5p14 and 5q12-q13. Genomics 19(1):31-37. https://doi.org/10. 1006/geno.1994.1008

Pérez CA, Stanley SA, Wysocki RW, Havranova J, Ahrens-Nicklas R, Onyimba F, Friedman JM (2011) Molecular annotation of integrative feeding neural circuits. Cell Metab 13(2):222-232. https://doi.org/10.1016/j.cmet.2010.12.013

Presse F, Nahon JL, Fischer WH, Vale W (1990) Structure of the human melanin concentrating hormone mRNA. Mol Endocrinol 4(4):632-637. https://doi.org/10.1210/mend-4-4-632 
Presse F, Conductier G, Rovere C, Nahon J (2014) The melanin-concentrating hormone receptors: neuronal and non-neuronal functions. Int J Obes Suppl 4:S31-S36. https://doi.org/10.1038/ ijosup.2014.9

Reti IM, Reddy R, Worley PF, Baraban JM (2002) Selective expression of Narp, a secreted neuronal pentraxin, in orexin neurons. J Neurochem 82(6):1561-1565. https://doi.org/10. 1046/j. 1471-4159.2002.01141.x

Risold P, Fellmann D, Lenys D, Bugnon C (1989) Coexistence of acetylcholinesterase-, human growth hormone-releasing factor (1-37)-, $\alpha$-melanotropin-and melanin-concentrating hormonelike immunoreactivities in neurons of the rat hypothalamus: a light and electron microscope study. Neurosci Lett 100(1):23-28. https://doi.org/10.1016/0304-3940(89)90654-X

Rodriguez M, Beauverger P, Naime I, Rique H, Ouvry C, Souchaud S, Dromaint S, Nagel N, Suply T, Audinot V (2001) Cloning and molecular characterization of the novel human melaninconcentrating hormone receptor MCH2. Mol Pharmacol 60(4):632-639. https://doi.org/10. 1210/mend-4-4-632

Rondini TA, Rodrigues Bde C, de Oliveira AP, Bittencourt JC, Elias CF (2007) Melaninconcentrating hormone is expressed in the laterodorsal tegmental nucleus only in female rats. Brain Res Bull 74(1-3):21-28. https://doi.org/10.1016/j.brainresbull.2007.04.006

Rondini TA, Donato J Jr, Rodrigues Bde C, Bittencourt JC, Elias CF (2010) Chemical identity and connections of medial preoptic area neurons expressing melanin-concentrating hormone during lactation. J Chem Neuroanat 39(1):51-62. https://doi.org/10.1016/j.jchemneu.2009.10.005

Rye DB (1997) Contributions of the pedunculopontine region to normal and altered REM sleep. Sleep 20(9):757-788. https://doi.org/10.1093/sleep/20.9.757

Sailer AW, Sano H, Zeng Z, McDonald TP, Pan J, Pong SS, Feighner SD, Tan CP, Fukami T, Iwaasa H, Hreniuk DL, Morin NR, Sadowski SJ, Ito M, Bansal A, Ky B, Figueroa DJ, Jiang Q, Austin CP, MacNeil DJ, Ishihara A, Ihara M, Kanatani A, Van der Ploeg LH, Howard AD, Liu Q (2001) Identification and characterization of a second melanin-concentrating hormone receptor, MCH-2R. Proc Natl Acad Sci USA 98(13):7564-7569. https://doi.org/10.1073/pnas. 121170598

Saito Y, Nothacker HP, Wang Z, Lin SH, Leslie F, Civelli O (1999) Molecular characterization of the melanin-concentrating-hormone receptor. Nature 400(6741):265-269. https://doi.org/10. $1038 / 22321$

Saito Y, Cheng M, Leslie FM, Civelli O (2001) Expression of the melanin-concentrating hormone $(\mathrm{MCH})$ receptor mRNA in the rat brain. J Comp Neurol 435(1):26-40. https://doi.org/10.1002/ cne. 1191

Sakurai T, Amemiya A, Ishii M, Matsuzaki I, Chemelli RM, Tanaka H, Williams SC, Richardson JA, Kozlowski GP, Wilson S (1998) Orexins and orexin receptors: a family of hypothalamic neuropeptides and $\mathrm{G}$ protein-coupled receptors that regulate feeding behavior. Cell 92 (4):573-585. https://doi.org/10.1016/S0092-8674(00)80949-6

Saper CB (2013) The neurobiology of sleep. Continuum: Lifelong Learn Neurol 19(1, Sleep Disorders):19-31. https://doi.org/10.1212/01.CON.0000427215.07715.73

Saper CB, Fuller PM (2017) Wake-sleep circuitry: an overview. Curr Opin Neurobiol 44:186-192. https://doi.org/10.1016/j.conb.2017.03.021

Saper CB, Swanson LW, Cowan WM (1979) An autoradiographic study of the efferent connections of the lateral hypothalamic area in the rat. J Comp Neurol 183(4):689-706. https://doi.org/10. 1002/cne.901830402

Saper CB, Akil H, Watson SJ (1986) Lateral hypothalamic innervation of the cerebral cortex: immunoreactive staining for a peptide resembling but immunochemically distinct from pituitary/arcuate alpha-melanocyte stimulating hormone. Brain Res Bull 16(1):107-120. https://doi. org/10.1016/0361-9230(86)90018-3

Shimomura Y, Mori M, Sugo T, Ishibashi Y, Abe M, Kurokawa T, Onda H, Nishimura O, Sumino Y, Fujino M (1999) Isolation and identification of melanin-concentrating hormone as the endogenous ligand of the SLC-1 receptor. Biochem Biophys Res Commun 261(3):622-626. https://doi.org/10.1006/bbrc.1999.1104 
Simerly R (2015) Organization of the hypothalamus. In: The rat nervous system, 4th edn. Elsevier, San Diego, pp 267-288

Sita LV, Diniz GB, Canteras NS, Xavier GF, Bittencourt JC (2016) Effect of intrahippocampal administration of anti-melanin-concentrating hormone on spatial food-seeking behavior in rats. Peptides 76:130-138. https://doi.org/10.1016/j.peptides.2015.12.007

Tan CP, Sano H, Iwaasa H, Pan J, Sailer AW, Hreniuk DL, Feighner SD, Palyha OC, Pong SS, Figueroa DJ, Austin CP, Jiang MM, Yu H, Ito J, Ito M, Guan XM, MacNeil DJ, Kanatani A, Van der Ploeg LH, Howard AD (2002) Melanin-concentrating hormone receptor subtypes 1 and 2: species-specific gene expression. Genomics 79(6):785-792. https://doi.org/10.1006/geno. 2002.6771

Tanabe A, Yanagiya T, Iida A, Saito S, Sekine A, Takahashi A, Nakamura T, Tsunoda T, Kamohara S, Nakata Y (2007) Functional single-nucleotide polymorphisms in the secretogranin III (SCG3) gene that form secretory granules with appetite-related neuropeptides are associated with obesity. J Clin Endocrinol Metabol 92(3):1145-1154. https://doi.org/10.1210/jc.2006-1808

Thompson RC, Watson SJ (1990) Nucleotide sequence and tissue-specific expression of the rat melanin concentrating hormone gene. DNA Cell Biol 9(9):637-645. https://doi.org/10.1089/ dna.1990.9.637

Toumaniantz G, Bittencourt JC, Nahon JL (1996) The rat melanin-concentrating hormone gene encodes an additional putative protein in a different reading frame. Endocrinology 137 (10):4518-4521. https://doi.org/10.1210/en.137.10.4518

Toumaniantz G, Ferreira PC, Allaeys I, Bittencourt JC, Nahon JL (2000) Differential neuronal expression and projections of melanin-concentrating hormone $(\mathrm{MCH})$ and $\mathrm{MCH}$-geneoverprinted-polypeptide (MGOP) in the rat brain. Eur J Neurosci 12(12):4367-4380. https://doi. org/10.1046/j.0953-816X.2000.01340.x

van den Pol AN, Acuna-Goycolea C, Clark KR, Ghosh PK (2004) Physiological properties of hypothalamic $\mathrm{MCH}$ neurons identified with selective expression of reporter gene after recombinant virus infection. Neuron 42(4):635-652. https://doi.org/10.1016/S0896-6273(04)00251-X

Vaughan JM, Fischer WH, Hoeger C, Rivier J, Vale W (1989) Characterization of melaninconcentrating hormone from rat hypothalamus. Endocrinology 125(3):1660-1665. https://doi. org/10.1210/endo-125-3-1660

Viale A, Zhixing Y, Breton C, Pedeutour F, Coquerel A, Jordan D, Nahon JL (1997) The melaninconcentrating hormone gene in human: flanking region analysis, fine chromosome mapping, and tissue-specific expression. Mol Brain Res 46(1-2):243-255. https://doi.org/10.1016/S0169328X(97)00018-1

Viale A, Ortola C, Hervieu G, Furuta M, Barbero P, Steiner DF, Seidah NG, Nahon J-L (1999) Cellular localization and role of prohormone convertases in the processing of pro-melanin concentrating hormone in mammals. J Biol Chem 274(10):6536-6545. https://doi.org/10. $1074 / j b c .274 .10 .6536$

Von Economo C (1930) Sleep as a problem of localization. J Nerv Ment Dis 71(3):249-259

Wang S, Behan J, O'Neill K, Weig B, Fried S, Laz T, Bayne M, Gustafson E, Hawes BE (2001) Identification and pharmacological characterization of a novel human melanin-concentrating hormone receptor, mch-r2. J Biol Chem 276(37):34664-34670. https://doi.org/10.1074/jbc. M102601200

Yao Y, Fu LY, Zhang X, van den Pol AN (2012) Vasopressin and oxytocin excite MCH neurons, but not other lateral hypothalamic GABA neurons. Am J Physiol Regul Integr Comp Physiol 302(7):R815-R824. https://doi.org/10.1152/ajpregu.00452.2011 
To whom it may concern,

We, Giovanne Baroni Diniz and Dr. Jackson Cioni Bittencourt, as authors of the book chapter entitled "Neuroanatomical Structure of the MCH System", published in the book MelaninConcentrating Hormone and Sleep, by Pandi-Perumal S., Torterolo P., Monti J. (eds) - Springer 2018, declare that this work was written in its totality by the joint effort of both authors. We ask, therefore, that to all intents, constructions, and purposes, it should be considered that both authors share the first authorship of the chapter.

Thank you for your consideration in this matter,
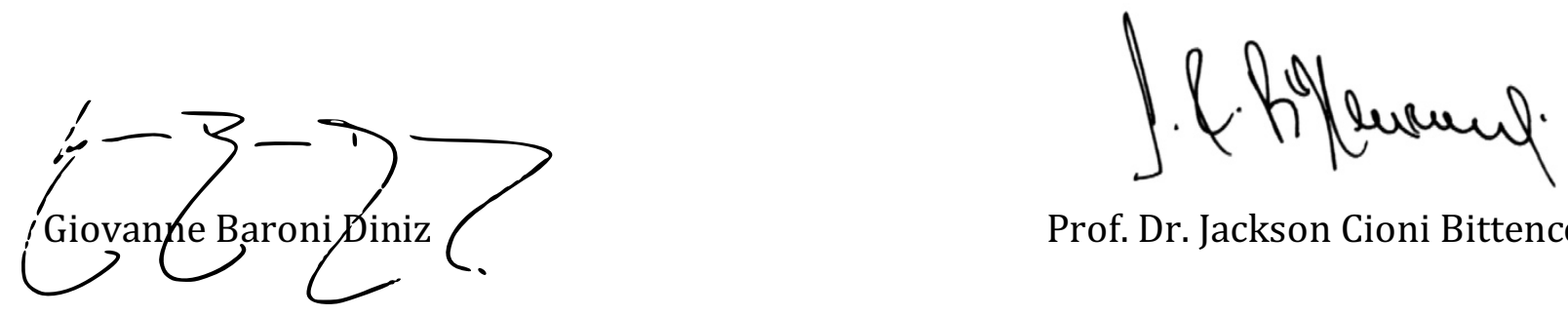

Prof. Dr. Jackson Cioni Bittencourt 



\section{Chapter 3}

The Melanin-Concentrating Hormone as an Integrative Peptide Driving Motivated Behaviors

\section{Diniz GB and Bittencourt JC}

As published in: Front. Syst. Neurosci. 11:32. 2017 DOI: 10.3389/fnsys.2017.00032 

OPEN ACCESS

Edited by:

Joel D. Hahn

University of Southern California,

United States

Reviewed by:

Scott E. Kanoski,

University of Southern California,

United States

Jessica R. Barson,

Drexel University College of Medicine

United States

*Correspondence:

Jackson C. Bittencourt

jcbitten@icb.usp.br

Received: 22 February 2017 Accepted: 04 May 2017

Published: 29 May 2017

Citation:

Diniz GB and Bittencourt JC (2017)

The Melanin-Concentrating Hormone as an Integrative Peptide Driving

Motivated Behaviors.

Front. Syst. Neurosci. 11:32 doi: 10.3389/fnsys.2017.00032

\section{The Melanin-Concentrating Hormone as an Integrative Peptide Driving Motivated Behaviors}

\author{
Giovanne B. Diniz ${ }^{1}$ and Jackson C. Bittencourt ${ }^{1,2 *}$ \\ 1 Laboratory of Chemical Neuroanatomy, Department of Anatomy, Institute of Biomedical Sciences, University of São Paulo, \\ São Paulo, Brazil, ${ }^{2}$ Center for Neuroscience and Behavior, Institute of Psychology, University of São Paulo, São Paulo, Brazil
}

The melanin-concentrating hormone $(\mathrm{MCH})$ is an important peptide implicated in the control of motivated behaviors. History, however, made this peptide first known for its participation in the control of skin pigmentation, from which its name derives. In addition to this peripheral role, $\mathrm{MCH}$ is strongly implicated in motivated behaviors, such as feeding, drinking, mating and, more recently, maternal behavior. It is suggested that $\mathrm{MCH}$ acts as an integrative peptide, converging sensory information and contributing to a general arousal of the organism. In this review, we will discuss the various aspects of energy homeostasis to which $\mathrm{MCH}$ has been associated to, focusing on the different inputs that feed the $\mathrm{MCH}$ peptidergic system with information regarding the homeostatic status of the organism and the exogenous sensory information that drives this system, as well as the outputs that allow $\mathrm{MCH}$ to act over a wide range of homeostatic and behavioral controls, highlighting the available morphological and hodological aspects that underlie these integrative actions. Besides the well-described role of $\mathrm{MCH}$ in feeding behavior, a prime example of hypothalamic-mediated integration, we will also examine those functions in which the participation of $\mathrm{MCH}$ has not yet been extensively characterized, including sexual, maternal, and defensive behaviors. We also evaluated the available data on the distribution of $\mathrm{MCH}$ and its function in the context of animals in their natural environment. Finally, we briefly comment on the evidence for $\mathrm{MCH}$ acting as a coordinator between different modalities of motivated behaviors, highlighting the most pressing open questions that are open for investigations and that could provide us with important insights about hypothalamic-dependent homeostatic integration.

Keywords: $\mathrm{MCH}$, hypothalamus, energy homeostasis, feeding behavior, reward, foraging, metabolism

\section{THE MELANIN-CONCENTRATING HORMONE[S]}

The existence of the $\mathrm{MCH}$ was postulated in the 1930s as a factor that induces pallor in the skin of amphibians and participates in these animals' mechanism of background color adaptation (Hogben and Slome, 1935). It took almost 50 years, however, for $\mathrm{MCH}$ to be isolated for the first time from the chum salmon (Oncorhynchus keta) hypophysis, where it plays exactly its hypothesized role in skin pigmentation by triggering a concentration of melanin in melanophores that results in pallor (Kawauchi et al., 1983). Although the chronology of MCH discovery emphasized its role in skin pigmentation, it is now proposed that this function only emerged for the first time in the 
last universal common ancestor of teleosts and holoceans, millions of years after the appearance of $\mathrm{MCH}$ synthesis in chordates (Kawauchi and Baker, 2004) .

It was later discovered that teleosts have two paralogs of $\mathrm{MCH}$, termed MCH1 and MCH2 (Ono et al., 1988), that have different patterns of distribution in the CNS. MCH1 corresponds

Abbreviations: 3V, third ventricle; 5-HT, serotonin; 5-HT? , unspecified serotonin receptor; $7 \mathrm{~N}$, nucleus of the cranial nerve VII; A10, ventral tegmental area dopaminergic group; A13, incerto-hypothalamic dopaminergic group; A8, retrorubral dopaminergic group; A9, substantia nigra dopaminergic group; ac, anterior commissure; Acb, nucleus accumbens; AcbC, nucleus accumbens, core subdivision; AcbSh, nucleus accumbens, shell subdivision; $\mathrm{ACh}$, acetylcholine; ADX, adrenalectomized; AgRP, agouti-related protein; $\mathrm{AH}$, adenohypophysis/anterior lobe of the pituitary; AHA, anterior hypothalamic area; AMPAR, AMPA ionotropic glutamate receptor; AMY, amylin; Amyg, amygdaloid nuclei; AON, anterior olfactory nucleus; AP, area postrema; Arc, arcuate nucleus; AV, anteroventral thalamic nucleus; AVP, vasopressin; AVPV, anteroventral periventricular nucleus; B7, dorsal raphe serotonergic group; B8, median raphe serotonergic group; BAT, brown adipose tissue; BNST, bed nucleus of the stria terminalis; CA, catecholamine; CA1, CA1 field of the hippocampus; CA2, CA2 field of the hippocampus; CA3, CA3 field of the hippocampus; CART, cocaine- and amphetamine-regulated transcript; $\mathrm{CB}_{1}$, cannabinoid receptor type 1 ; cc, corpus callosum; $\mathrm{CeA}$, central nucleus of the amygdala; $\mathrm{CG}$, central gray of the spinal cord; $\mathrm{CgCx}$, cingulate cortex; CORT, cortisol/corticosterone; cPPTg, caudal pedunculopontine tegmental nucleus; $\mathrm{CPu}$, caudate putamen; $\mathrm{CRF}$, corticotrophin-releasing factor; $\mathrm{D}_{1}$, dopamine receptor subtype $1 ; \mathrm{D}_{2}$, dopamine receptor subtype 2; DA, dopamine; DG, dentate gyrus; $\mathrm{DMH}$, dorsomedial hypothalamic nucleus; DR, dorsal raphe nucleus; DTM, dorsal tuberomammillary nucleus; Dyn, dynorphins; EB, estradiol benzoate; ER $\alpha$, estrogen receptor type $\alpha$; EW, Edinger-Westphal nucleus; EWcp, centrally projecting EdingerWestphal nucleus; $\mathrm{f}$, fornix; FOS, FOS proto-oncogene, AP-1 transcription factor subunit; $\mathrm{GABA}_{\mathrm{A}}$, GABA ionotropic receptor; GIRK, $\mathrm{G}$ protein-coupled inwardly rectifying $\mathrm{K}^{+}$; GLUT3, glucose transporter 3; GnRH, gonadotropin-releasing hormone; GP, globus pallidus; Hab, habenular nuclei; Hcrt, Orexin/hypocretin gene; HF, hippocampal formation; HPA, hypothalamus-pituitary-adrenal; HPT, hypothalamus-pituitary-thyroid; Hyp, hypothalamic nuclei; IC, inferior colliculus; IHy, incerto-hypothalamic area; ImC, intermediolateral column of the spinal cord; Ins, insular cortex; IR, insulin receptor; $\mathrm{K}_{\mathrm{ATP}}$, ATP-sensitive potassium channel; $\mathrm{LD}$, laterodorsal thalamic nucleus; LDTg, laterodorsal tegmental nucleus; Lep, leptin gene; LepR, leptin receptor; $L e p R$, leptin receptor gene; LG, lateral geniculate nucleus; LH, luteinizing hormone; LHA, lateral hypothalamic area; LHAa, anterior part of the LHA; LHAp, posterior part of the LHA; LM, lateral mammillary nucleus; LPOA, lateral preoptic area; LS, lateral septal nucleus; $\mathrm{mACh}$, metabotropic $\mathrm{ACh}$ receptor; MAS, masseter muscle; $\mathrm{MCH}$, melanin-concentrating hormone; $\mathrm{MCH} 1$, teleost melanin-concentrating hormone subtype 1; $\mathrm{MCH} 2$, teleost melaninconcentrating hormone subtype 2; MCHR1, MCH receptor type 1; Mchr1, MCH receptor 1 gene; $\mathrm{MCx}$, motor cortex; $\mathrm{MD}$, mediodorsal thalamic nucleus; $\mathrm{ME}$, median eminence; MEe, external layer of the median eminence; $\mathrm{MEi}$, internal layer of the median eminence; mfb, medial forebrain bundle; MG, medial geniculate nucleus; mGlu, unspecified metabotropic GLU receptor; mGlu1, metabotropic GLU receptor subtype 1; mGlu5, metabotropic GLU receptor subtype 5; MidThal, midline thalamic nuclei; ml, medial lemniscus; MM, medial mammillary nucleus; $\mathrm{MnR}$, median raphe nucleus; MoV, motor nucleus of the V nerve; MPOA, medial preoptic area; MRt, medullary reticular formation; MS, medial septal nucleus; MSN, medium spiny neuron; N/OFQ, nociceptin/orphanin FQ; NCX, $\mathrm{Na}^{+} / \mathrm{Ca}^{2+}$ exchanger; NE, norepinephrine; NEI, neuropeptide E-I; NGE, neuropeptide G-E; $\mathrm{NH}$, neurohypophysis/posterior lobe of the pituitary; NMDAR, NMDA ionotropic GLU receptor; NOP, nociceptin/orphanin Q receptor; NPY, neuropeptide Y; NREM, non-rapid eye movement sleep; NTS, nucleus of the solitary tract; Nts, neurotensin; OB, main olfactory bulb; opt, optic tract; ORX, orexins/hypocretins; $\mathrm{ORX}_{\mathrm{A}}$, orexin $\mathrm{A} /$ hypocretin-1; ORX $\mathrm{B}$, orexin B/hypocretin-2; ORXR, unspecified orexin receptor; $\mathrm{ORXR}_{2}$, orexin receptor type 2; OT, olfactory tubercle; OXT, oxytocin; OXTR, oxytocin receptor; OVX, ovariectomized; $\mathrm{P}_{4}$, progesterone; $\mathrm{P} 2$ ?, unspecified purinergic receptor; PAG, periaqueductal gray matter; PAGvl, ventrolateral periaqueductal gray matter; PAGdm, dorsomedial periaqueductal gray matter; $\mathrm{PB}$, parabrachial nucleus; Pe, periventricular hypothalamic nucleus; $\mathrm{PaF}$, parafascicular thalamic nucleus; PHA, posterior hypothalamic area; PI3K, phosphatidylinositol 3-kinase; PIP3, phosphatidylinositol-3,4,5-trisphosphate; PKA, protein kinase A; PKC, protein kinase C; PLC, phospholipase C; Pmch, Pro-melanin-concentrating hormone gene; PnRt, pontine reticular to the peptide isolated by Kawauchi et al. (1983) and is mostly associated with the control of skin pigmentation. Berman et al. (2009), working with the zebrafish (Danio rerio) hypothalamus, demonstrated that $\mathrm{MCH} 1$ immunoreactivity is largely impacted by background color, reflecting its role in skin pigmentation, while only $\mathrm{MCH} 2$ displays pronounced alterations in fasted animals. This correlation between hypothalamic $\mathrm{MCH} 2$ and the energy balance of the organism indicates that $\mathrm{MCH}$ may be linked to motivated behaviors and integrative functions at least since the divergence of tetrapods and ray-finned fishes at $\sim 440$ million years ago (Amores et al., 2011).

Six years after the discovery of MCH1 in the salmon hypophysis, Nahon et al. (1989) and Vaughan et al. (1989) described for the first time the mammalian $\mathrm{MCH}$ coding gene and structure. Using rat samples, these researchers showed that there is a single mammalian $\mathrm{MCH}$, with 19 amino acids and a cysteine bridge that confers it a cyclic structure, synthesized from a 165 amino acids precursor coded by the Pmch gene. This precursor also contains the sequence of two other peptides, NEI and NGE, and although some functions are now attributed to the former (for a review, see Bittencourt and Celis, 2008), the biological activity of the latter is largely unknown. Synteny, genomic structure, and sequence identity indicate that the mammalian $\mathrm{MCH}$ is an ortholog of teleost MCH2 (Berman et al., 2009) and shows remarkable conservation when compared to the single $\mathrm{MCH}$ of a cartilaginous fish, the scalloped hammerhead shark (Sphyrna lewini), with a single substitution at the C-Terminal (Mizusawa et al., 2012).

\section{ANATOMICAL ASPECTS}

The synthesis of MCH shows two remarkably conserved features: most, if not all, MCH-immunoreactive (MCH-ir) neurons are found in the hypothalamus, and those neurons have widespread projections throughout the CNS. The first anatomical characterization of $\mathrm{MCH}$ was made in the male and female rat by Bittencourt et al. (1992). In this species, most MCH-ir neurons are found in the LHA and, to a lesser extent, in the

formation; Po, posterior thalamic complex; POMC, proopiomelanocortin; PPTg, pedunculopontine tegmental nucleus; Pr, prepositus nucleus; PreCBL, precerebellar nuclei; Pretect, pretectal nuclei; PV, paraventricular thalamic nucleus; $\mathrm{PVH}$, paraventricular hypothalamic nucleus; PVHp, parvocellular subdivision of the paraventricular hypothalamic nucleus; PVHm, magnocellular subdivision of the paraventricular hypothalamic nucleus; Pir, piriform cortex; REM, rapid eye movement sleep; RIA, radioimmunoassay; rPPTg, rostral pedunculopontine tegmental nucleus; SAL, salivary glands; SuS, superior salivatory nucleus; SC, superior colliculus; scp, superior cerebellar peduncle; SHi, septohippocampal nucleus; SM, supramammillary nucleus; SN, substantia nigra; SpCd, spinal cord; SpV, spinal trigeminal nucleus; SSCx, somatosensory cortex; st, stria terminalis; SubThal, subthalamus; TRH, thyrotropin-releasing hormone; TRHR, unspecified thyrotropin-releasing hormone receptor; TRPC, transient receptor potential channel; TSH, thyroid-stimulating hormone; UCN1, urocortin 1; UCN2, urocortin 2; UCN3, urocortin 3; V1a, vasopressin receptor subtype 1a; VDCC, voltage-dependent calcium channel; Vest, vestibular nuclei; VL, ventrolateral thalamic nucleus; VMH, ventromedial hypothalamic nucleus; VTA, ventral tegmental area; YR, unspecified NPY receptor; ZI, zona incerta; $\alpha-\mathrm{MSH}$, $\alpha$-melanocyte-stimulating hormone; $\alpha \mathrm{R}$, unspecified adrenergic receptor; $\alpha 2_{\mathrm{A}}$, adrenergic receptor type $2 \mathrm{~A} ; \mathrm{\kappa OR}$, opioid receptor type $\kappa$. 


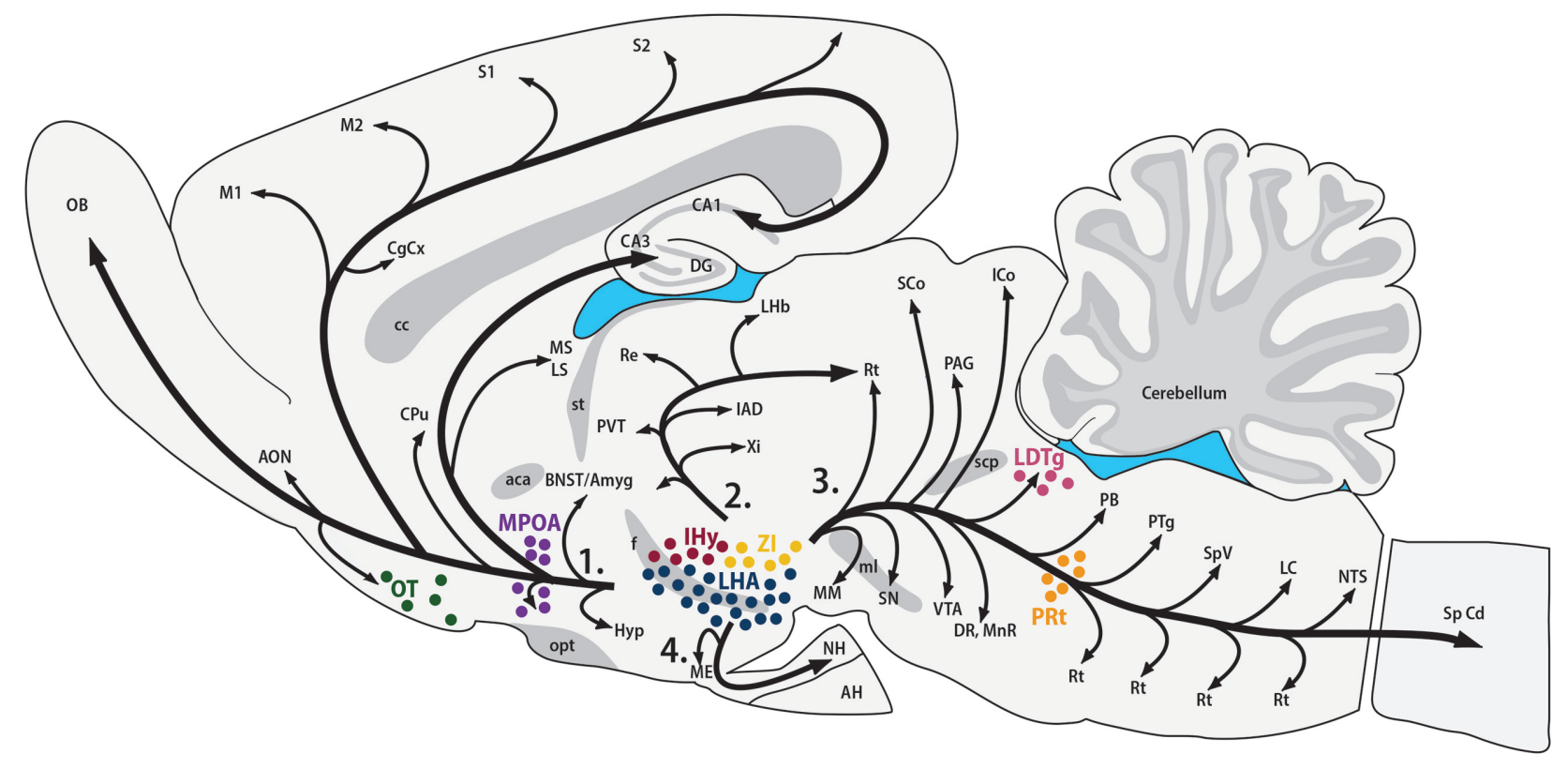

FIGURE 1 | MCH synthesis is found in discrete areas of the rat nervous system. Sagittal representation of the rat central nervous system illustrating the main areas where $\mathrm{MCH}$-ir neurons are found. The hypothalamic areas of synthesis in all animals are: the LHA (blue) and the IHy (red). Extra-hypothalamic areas are: the ZI (yellow), the OT (green) and the paramedian PnRt (orange). Exclusively in female rats, one extra-hypothalamic site can be found, in the laterodorsal tegmental nucleus (pink). During the lactation period, $\mathrm{MCH}$-immunoreactive neurons can be detected in the medial preoptic area (purple). The four main pathways of $\mathrm{MCH}$-ir fibers are also represented: 1 - ascending pathway; 2 - periventricular pathway; 3 - descending pathway; and 4 - hypophysaire pathway.

AHA and PHA of the medial zone and in the dorsalmost area of the periventricular zone. A second group of rostral neurons is found in the IHy, previously known as rostromedial ZI (Bittencourt et al., 1992; Murray et al., 2000c), where MCH-ir neurons are intermingled with the DA neurons of the A13 group (Sita et al., 2003, 2007). Exclusively during the postpartum period, immunoreactivity to $\mathrm{MCH}$ can be detected in neurons of the MPOA and at the rostralmost aspect of the PVH, with an intensity that follows the progression of lactation and, with weaning, disappears (Knollema et al., 1992; Rondini et al., 2010). Outside the hypothalamus, $\mathrm{MCH}$-ir neurons were described in the OT, ZI, and paramedian PnRt (Bittencourt et al., 1992) and in the LDTg of the female rat (Rondini et al., 2007). Little is known about the role played by $\mathrm{MCH}$ in these extrahypothalamic areas. Figure 1 summarizes the loci of $\mathrm{MCH}$ synthesis in the rat CNS and the main pathways formed by $\mathrm{MCH}$-ir fibers.

Contrasting to the highly restricted pattern of $\mathrm{MCH}$ synthesis, $\mathrm{MCH}$-ir fibers are found widespread throughout the whole CNS. Among the regions that contain fibers are olfactory areas, the septal nuclei, the basal nuclei, the HF, the neocortex, several diencephalic nuclei, the mesencephalic and PnRt, the PAG and all levels of the $\mathrm{SpCd}$. The regions with the sparsest presence $\mathrm{MCH}$-ir fibers are the cerebellum and some motor nuclei of the brainstem. In most of these areas, $\mathrm{MCH}$-ir fibers are varicose and present many boutons en passage and boutons terminaux, suggesting an extensive synaptic activity (Bittencourt et al., 1992). Figure $2 \mathrm{~A}$ is a visual representation of the $\mathrm{MCH}$-ir fiber density in different areas of the rat CNS.
The distribution of MCH-ir cells has been studied in a wide range of animals, including agnathans (Mizuno, 1991; Bird et al., 2001), cartilaginous fish (elasmobranchii) (Vallarino et al., 1989; Mizusawa et al., 2012), teleosts (Naito et al., 1985; Batten and Baker, 1988; Powell and Baker, 1988; Bird and Baker, 1989; Minth et al., 1989; Gröneveld et al., 1995; Mancera and FernandezLlebrez, 1995; Amano et al., 2003; Huesa et al., 2005; Matsuda et al., 2009; Suzuki and Yamamoto, 2013), anurans (Andersen et al., 1986; Francis and Baker, 1995; Lázár et al., 2002), reptiles (Cardot et al., 1994), and birds (Cardot et al., 1998, 1999). In mammals, in addition to the rat (Bittencourt et al., 1992), MCH-ir cell bodies have been mapped in the mouse (Elias et al., 2001), Djungarian hamster (Khorooshi and Klingenspor, 2005), Syrian hamster (Vidal et al., 2005), tufted capuchin monkey (Bittencourt et al., 1998), humans (Elias et al., 2001), pigs (Chometton et al., 2014), sheep (Tillet et al., 1996), and cats (Torterolo et al., 2006). For a review of the anatomical aspects of $\mathrm{MCH}$ in mammals, see Bittencourt (2011).

Albeit there are some differences between these animals, the main locus of $\mathrm{MCH}$ synthesis in all of them is the hypothalamus or homologous structures, indicating that $\mathrm{MCH}$-ir neurons reside this region at least since the emergence of the last vertebrate common ancestor. There is also a remarkable conservation in the widespread distribution of $\mathrm{MCH}$-ir fibers. The hypothalamus, through its extensive projections, is a key structure for the execution of motivated behaviors, as understood as behavioral programs directed to exogenous factors that are executed by animals and result in a benefit to the survival of the organism or of its species (Swanson, 2005). This long phylogenetic history 


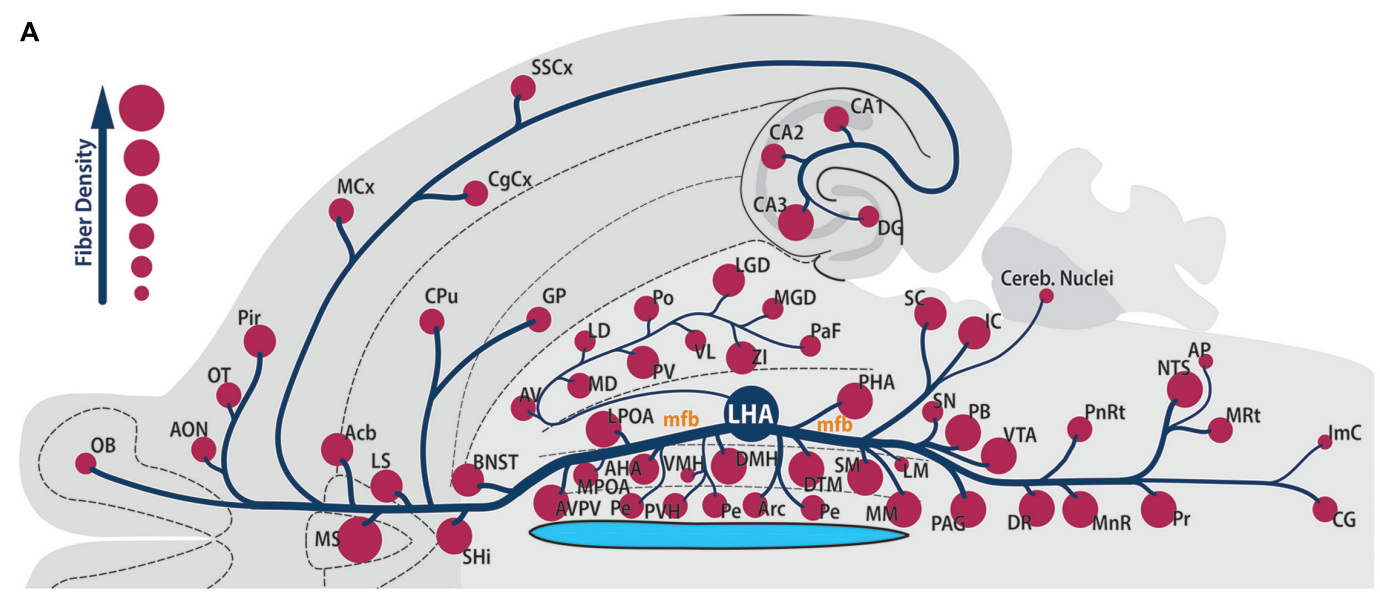

B

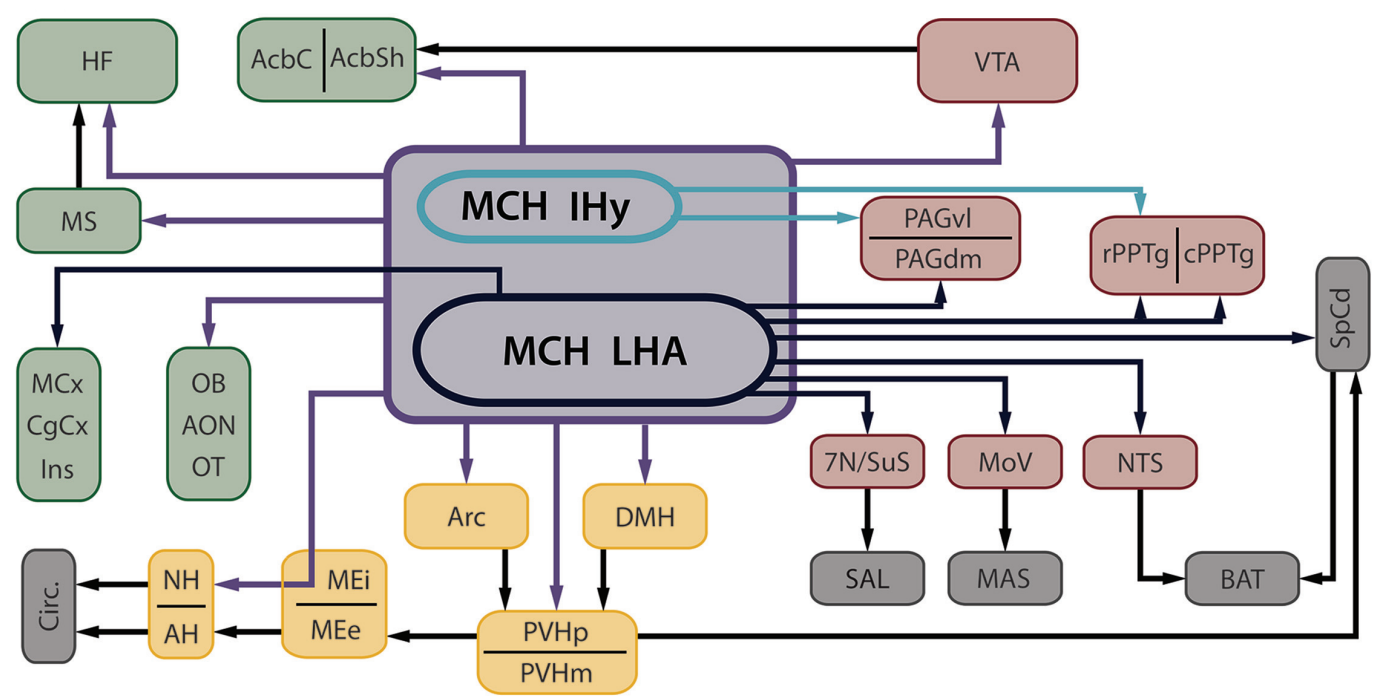

FIGURE 2 | MCH-immunoreactive fibers are profusely distributed in the neuroaxis and are found in several areas related to feeding behavior and energy homeostasis. (A) Flatmap representation of the rat central nervous system illustrating the main areas where $\mathrm{MCH}$-ir fibers can be found. The relative density of $\mathrm{MCH}$-ir fibers in each area is indicated by the size of the circles. Observe that septal, hypothalamic and brainstem areas are densely innervated by $\mathrm{MCH}$ neurons, while cortical and thalamic areas display a smaller number of fibers. Due to the position of the LHA, most of the MCH-ir fibers leave this region intermingled to the $\mathrm{mfb}$, with the periventricular pathway as a notable exception to this rule. (B) Schematic representation of the main targets of $\mathrm{MCH}$-ir fibers in the rat $\mathrm{CNS}$. Dark blue arrows represent projections originated from $\mathrm{MCH}$-ir neurons located in the LHA, while green arrows indicate projections preferentially originated from the IHy. Purple arrows indicate projections originated from both areas or a lack of hodological studies to pinpoint the origin of such projections. Dark arrows indicate indirect connections relevant to this circuit. The main targets of $\mathrm{MCH}$ innervation can be arranged in three groups: telencephalic projections, in green; hypothalamic-hypophysaire projections, in yellow; and brainstem projections, in red. Downstream targets of $\mathrm{MCH}$ action are represented in gray.

accounts, at least partially, for the numerous functions on which $\mathrm{MCH}$ has been suggested to play a role in, and may explain how $\mathrm{MCH}$ acquired an important integrative role in motivated behaviors centered on its hypothalamic localization supported by a widespread fiber distribution.

\section{FEEDING BEHAVIOR}

The most thoroughly described function of $\mathrm{MCH}$ is on the modulation of feeding behavior. The injection of $\mathrm{MCH}$ in the ventricle of rats (Qu et al., 1996; Rossi et al., 1997; Della-Zuana et al., 2002; Clegg et al., 2003; Duncan et al., 2005; Sakamaki et al., 2005) and mice (Gomori et al., 2003; Glick et al., 2009), as well as in the PVH (Rossi et al., 1997) or in the Acb of rats (Georgescu et al., 2005; Mul et al., 2011), provokes an increase in chow consumption followed by a gain of body weight and fat mass. Rossi et al. (1997), however, demonstrated that only acute injections of $\mathrm{MCH}$ can induce these effects, while chronic administration is unable to maintain it. Injections of $\mathrm{MCH}$ in the $3 \mathrm{~V}$ also induce an increase in water and saccharin consumption in rats, independently of chow availability, indicating that $\mathrm{MCH}$ participates in hydric homeostasis processes (Clegg et al., 2003; Sakamaki et al., 2005; Furudono et al., 2006). This same 
treatment, however, increases alcohol consumption, suggesting that $\mathrm{MCH}$ participates in hedonistic aspects of this behavior as well (Duncan et al., 2005). Mice deficient in leptin synthesis $\left[\right.$ Lep $\left.{ }^{\mathrm{ob} / \mathrm{ob}}\right]$ with an obese phenotype display a higher expression of Pmch mRNA in the LHA when compared to WT mice, and this expression increases following fasting (Qu et al., 1996). $\mathrm{MCH}$-overexpressing mice display higher chow consumption and increased body weight, as well as circulating levels of leptin and insulin resistance (Ludwig et al., 2001).

Data obtained from genetic models started broadening the roles associated to MCH. Shimada et al. (1998) report that $\mathrm{Pmch}^{-1-}$ mice are leaner than $\mathrm{Pmch}^{+}$cohorts, but several evidence point to this leaner phenotype as the result of multiple physiological alterations. Not only $\mathrm{Pmch}^{-/-}$animals eat less, but they displayed increased oxygen consumption rates; their ability to overeat in response to starvation is not affected, although $\mathrm{Pmch}^{-1-}$ animals have greater weight loss and higher mortality rate; and restricted food availability resulted in similar decreases in food intake between $\mathrm{Pmch}^{-/-}$and $\mathrm{Pmch}^{+}$mice, but deficient animals displayed increased weight loss. Another important evidence came with the work of Marsh et al. (2002), that reports that MCHR1-deficient mice have increased locomotor activity and energy expenditure that underlie an apparently contradictory hyperphagia observed in these animals. Finally, $\mathrm{Lep}^{\mathrm{ob} / \mathrm{ob}} \mathrm{Pmch}^{-/-}$mice are leaner than $\mathrm{Lep}^{\mathrm{ob} / \mathrm{ob}} \mathrm{Pmch}^{+}$, but their food consumption is not decreased (Segal-Lieberman et al., 2003). This leaner phenotype is attributed to factors linked to energy expenditure, with higher motor activity and basal temperature in response to cold. Taken together, these results indicate that $\mathrm{MCH}$ acts on energy homeostasis through multiple pathways, including energy expenditure, locomotor activity and reward, while increases in ingestive behavior promoted by $\mathrm{MCH}$ are secondary in nature. In this section, we will review the multiple aspects of $\mathrm{MCH}$ action over nutritional homeostasis.

Although variations in dietary parameters are capable of modulating $\mathrm{MCH}$ neurons, with fasting increasing Pmch expression, variations in this expression may also underlie the preference of some animals for a specific diet. For example, Morganstern et al. (2010b) identified a subset of Sprague-Dawley rats that are prone to overconsume a high-fat diet (HFD) when compared to animals that show a baseline consumption of HFD. These authors demonstrated that animals that ingest a higher amount of HFD during a 5-day period (as measured by caloric intake) have increased levels of Pmch expression in the LHA when compared to animals that were kept in the same diet but ingested a lower number of calories. This difference persists even after the animals have been maintained in regular chow for weeks, suggesting that this intrinsic variation in Pmch levels may generate the preference of these animals for the higher fat content or increased palatability of the diet. This is extremely relevant because a similar mechanism may underlie human pathologies related to the excessive consumption of fat-rich foods. To explore alterations in the $\mathrm{MCH}$ peptidergic system in other models of natural or induced preference may generate important knowledge on the human physiology of ingestive behaviors (Barson et al., 2012).

\section{Inputs}

Melanin-concentrating hormone neurons are influenced by many inputs, including other neurons in the LHA, adjoining hypothalamic nuclei, telencephalic and brainstem areas, in addition to circulating factors that act as metabolic cues. Figure 3 summarizes the main homeostatic-relevant inputs to $\mathrm{MCH}$ neurons.

\section{Lateral Hypothalamic Area}

The LHA is classically characterized by two populations of neurons, one that is MCH-ir (Bittencourt et al., 1992) and one that is ORX-ir (Peyron et al., 1998), both intermingled with GABAergic and GLUergic interneurons (Gao and van den Pol, 2001) who provide extensive input to $\mathrm{MCH}$ neurons through ionic and metabotropic channels (Gao and van den Pol, 2001; Huang and van den Pol, 2007). These two populations coexist in the LHA but are almost completely segregated, with a minimal number of neurons that co-synthesize these two peptides (Broberger et al., 1998; Elias et al., 1998; Peyron et al., 1998). There are two orexin peptides, $\mathrm{ORX}_{\mathrm{A}}$ and $\mathrm{ORX}_{\mathrm{B}}$, coded by the same gene (Hcrt) and involved in multiple functions related to homeostatic control and arousal (for recent reviews, see Mahler et al., 2014 and Sakurai, 2014). Both MCH and ORX neurons have profuse immunoreactive fibers in the LHA, contacting numerous neighboring cells (Bittencourt et al., 1992; Peyron et al., 1998). Although the high density of MCH-ir fibers close to $\mathrm{MCH}$-ir cells could point to a recurrent effect of $\mathrm{MCH}$ on $\mathrm{MCH}$ cells, probably inhibitory due the nature of $\mathrm{MCH}$ neurons (Gao and van den Pol, 2001), there are no alterations in the electrophysiological properties of $\mathrm{MCH}$ neurons after $\mathrm{MCH}$ application in LHA slices (van den Pol et al., 2004).

$\mathrm{MCH}-\mathrm{ir}$ neurons are densely contacted by ORX neurons, with some single $\mathrm{MCH}$-ir cells receiving contacts by up to 30 individual ORX-ir boutons. Both $\mathrm{ORX}_{\mathrm{A}}$ and $\mathrm{ORX}_{\mathrm{B}}$ have a postsynaptic excitatory effect over $\mathrm{MCH}$ cells, depolarizing their membrane potential and increasing spike frequency (Figure 4A). $\mathrm{ORX}_{\mathrm{B}}$ has also a presynaptic effect, increasing GLUergic input to $\mathrm{MCH}$ cells (van den Pol et al., 2004) (Figure 5). The similar response amplitude of $\mathrm{MCH}$ neurons to $\mathrm{ORX}_{\mathrm{A}}$ and $\mathrm{ORX}_{\mathrm{B}}$ suggests that this signaling occurs through $\mathrm{ORXR}_{2}$, as $\mathrm{ORXR}_{1}$ responds poorly to $\mathrm{ORX}_{\mathrm{B}}$ (Sakurai et al., 1998). Apergis-Schoute et al. (2015), however, provided evidence that the relationship between ORX and MCH may be more complex, since optogenetic stimulation of ORX neurons in LHA slices promoted inhibition of some $\mathrm{MCH}$ neurons through a presynaptic mechanism, increasing GABAergic input on $\mathrm{MCH}$ cells, while other $\mathrm{MCH}$ neurons were excited by ORX stimulus. Regardless, all these results suggest that these two LHA populations are connected, with ORX influencing close $\mathrm{MCH}$ neurons in multiple ways.

While some MCH neurons co-synthesize the CART (Elias et al., 2001) and nesfatin (Brailoiu et al., 2007; Fort et al., 2008), almost all ORX neurons co-synthesize Dyn or N/OFQ, both endogenous opioids (Maolood and Meister, 2010). The application of Dyn or N/OFQ hyperpolarizes $\mathrm{MCH}$ cells, increasing their input resistance ( $\mathrm{Li}$ and van den Pol, 2006;

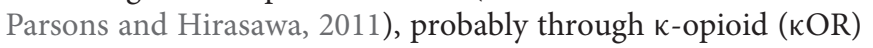
and NOP receptors present in these cells (Parks et al., 2014) 


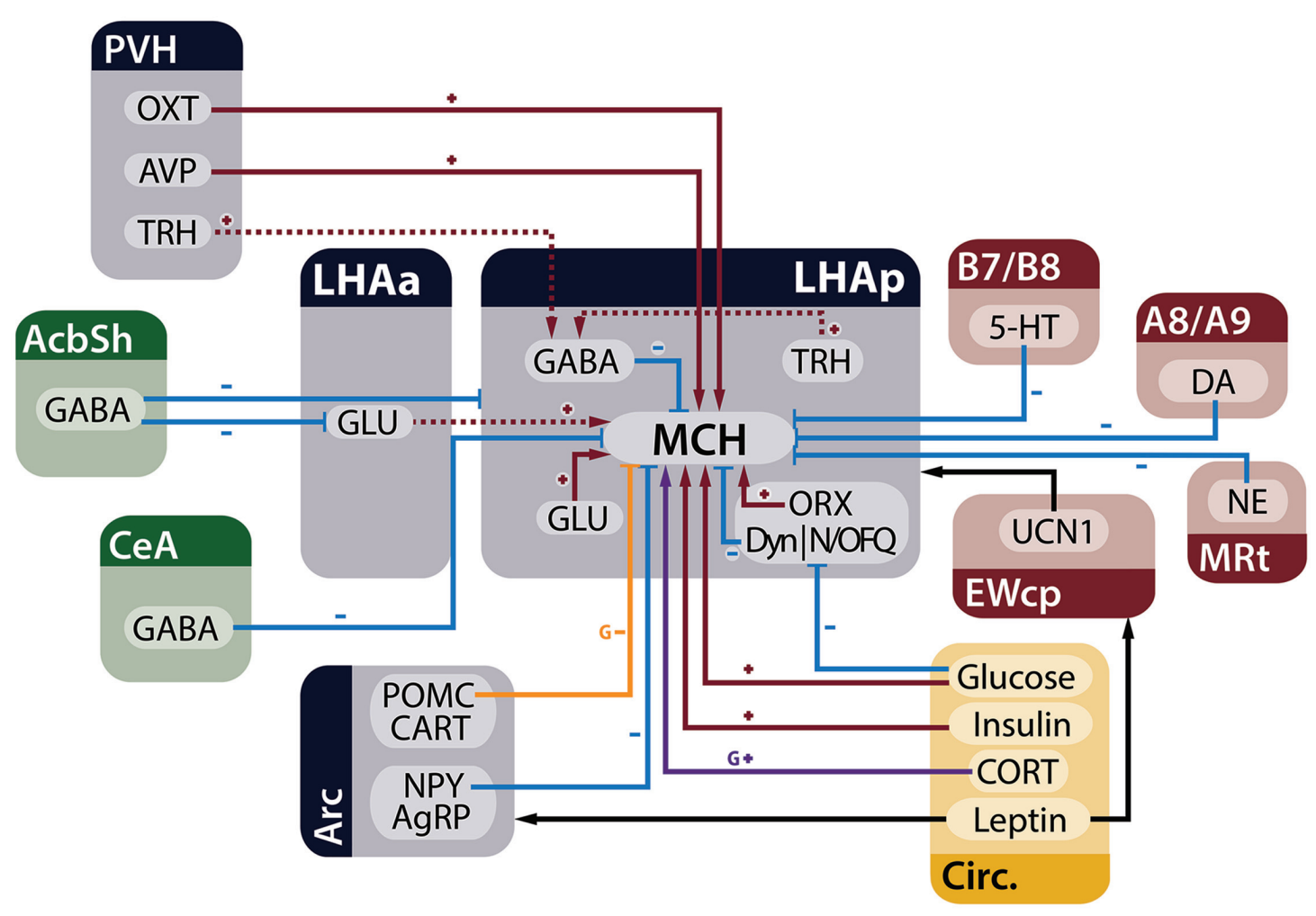

FIGURE 3 | MCH neurons receive several inputs relevant to the control of energy homeostasis. Neurons from the telencephalon (green), hypothalamus (blue), and brainstem (red), in addition to circulating factors (yellow), are capable of modulating electrophysiological and genetic expression properties of $\mathrm{MCH}$ neurons in the LHA. Red arrows with plus signs represent depolarizing inputs, blue arrows with minus signs represent hyperpolarizing inputs, black arrows indicate relevant indirect connections to the circuit, the purple arrow represents increased expression of the Pmch gene and the orange arrow an input that decreases gene expression. Dashed lines indicate connections that have ample physiological support but lack morphological demonstration or may be composed of multiple polysynaptic elements. Some presynaptic modulatory inputs to GABA- and GLUergic neurons were omitted for clarity.

(Figure 4B). These results suggest that $\mathrm{MCH}$ neurons could potentially be stimulated by ORX and inhibited by Dyn and N/OFQ originated from the same fibers. Li and van den Pol (2006) and Parsons and Hirasawa (2011) report that MCH cells quickly desensitize to Dyn and N/OFQ, while the response to ORX remains largely unaltered after sequential applications. Through this mechanism, Dyn and N/OFQ could damp the MCH response to brief spontaneous activation of ORX cells, since only constant ORX input would surpass the desensitized inhibitory effect of Dyn and N/OFQ and activate MCH cells. In other words, these endogenous opioids provide the ORX-MCH circuit with a mechanism for noise filtering.

In addition to the chiefly LHA-centered $\mathrm{MCH}$ and ORX populations, neurons with distinct chemical signatures and broader distributions are also found in the LHA. One example is the TRH-ir neuronal population located in the LHA, that does not co-localize with MCH or ORX (Horjales-Araujo et al., 2014) and can also be identified in the PVH, VMH, DMH, suprachiasmatic, Pe and Arc hypothalamic nuclei (Brownstein et al., 1974). Besides its role in thyroid function regulation through the $\mathrm{AH}, \mathrm{TRH}$ is also associated with the control of energy homeostasis through central pathways: icv injections decrease feeding and increase thermogenesis and locomotor activity (for a review on TRH functions, see Lechan and Fekete, 2006). The central effects of TRH are all antagonistic to $\mathrm{MCH}$ actions, suggesting that TRH-mediated inhibition of $\mathrm{MCH}$ neurons could account, at least partially, for the observed results. Indeed, Zhang and van den Pol (2012) report that TRH inhibit $\mathrm{MCH}$ neurons by increasing presynaptic GABAergic input to $\mathrm{MCH}$ neurons (Figure 5). In conclusion, $\mathrm{TRH}$ may modulate feeding behavior and energy expenditure by inhibiting $\mathrm{MCH}$ neurons in the LHA, and the source of this TRH input may be from the LHA itself or from other hypothalamic nuclei linked to energy balance, as the PVH, VMH, DMH, and Arc.

Recently, another population of LHA neurons has emerged an important mediator of energy homeostasis: Nts neurons that also contain LepR, known as Nts-LepR. These neurons are found in the LHA (Leinninger et al., 2011) and make synaptic contacts with local neurons (Louis et al., 2010; Leinninger et al., 2011). Although Leinninger et al. (2011) have shown that Nts-LepR neurons make inhibitory synaptic contacts with ORX neurons, but not $\mathrm{MCH}$ neurons, it is possible that a polysynaptic circuit may link Nts-LepR neurons to $\mathrm{MCH}$. The selective ablation of LHA Nts-LepR neurons leads to a phenotype of increased body weight, but these mice have only a slightly 

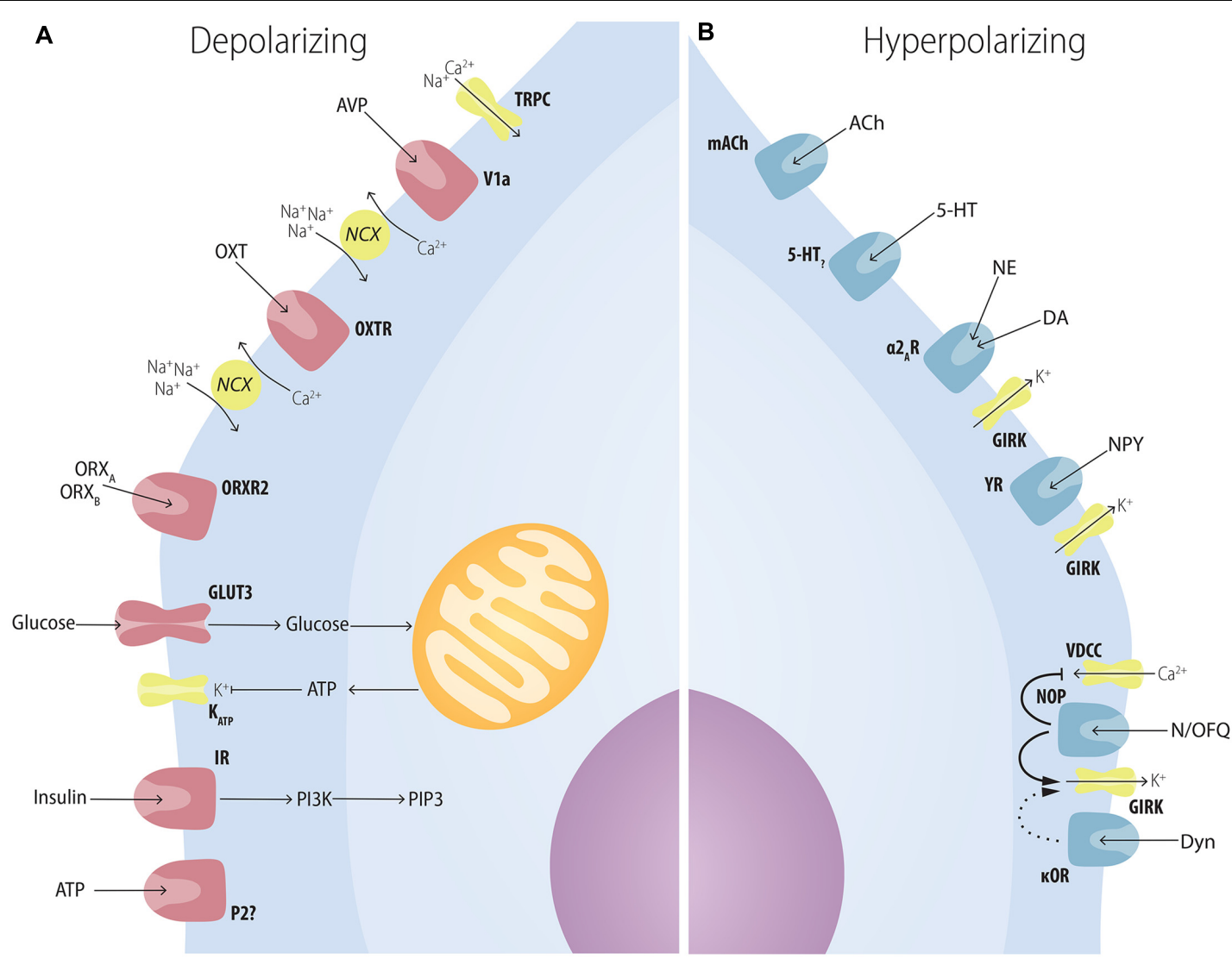

FIGURE 4 | Multiple factors have postsynaptic effects on $\mathbf{M C H}$ neurons. (A) Several neuropeptides increase the excitability of $\mathrm{MCH}$ neurons through postsynaptic mechanisms. AVP depolarizes MCH neurons through NCXs and TRPC; OXT increases Na ${ }^{+}$currents through NCXs; ATP in the extracellular medium increases $\mathrm{MCH}$ excitability through unknown mechanisms that may depend on $\mathrm{P} 2$ ?; orexins depolarize $\mathrm{MCH}$ neurons with special affinity for $\mathrm{ORX} \mathrm{B}_{\mathrm{B}}$, suggesting an $\mathrm{ORXR}_{2}$-dependent pathway; glucose increases neuronal excitability in a mechanism similar to pancreatic $\beta$-cells: GLUT3 transporters increase the amount of glucose in the intracellular medium, boosting the amount of produced ATP, what blocks ATP-sensitive potassium channels (K $\mathrm{K}_{\text {ATP) }}$; insulin excites MCH neurons and activates PIP3-dependent pathways. (B) Several neuropeptides decrease the excitability of $\mathrm{MCH}$ neurons through postsynaptic mechanisms. ACh and 5-HT, two important elements of the central arousal system, inhibit $\mathrm{MCH}$ neurons; both $\mathrm{NE}$ and DA are capable of decreasing $\mathrm{MCH}$ excitability through the activation of the adrenoceptor type $2 \mathrm{~A}$ and resulting activation of $\mathrm{K}^{+}$currents through GIRK channels; NPY hyperpolarizes MCH neurons also through GIRK currents; opioids such as N/OFQ and Dyn hyperpolarize MCH neurons with convergent mechanisms through the blockage of VDCC and an increase in GIRK currents.

increased food consumption when young. On the other hand, these animals have increased adiposity and decreased $\mathrm{VO}_{2}$ and ambulatory activity (Leinninger et al., 2011). This phenotype inversely mirrors that observed in $\mathrm{Pmch}^{-/-}$and $\mathrm{Mchrl}^{-/-}$ mice, that show a reduction in chow consumption that is too small to explain their weight deficit, increased $\mathrm{O}_{2}$ consumption and increased ambulatory activity (Shimada et al., 1998; Marsh et al., 2002). These energy expenditure alterations seen in NtsLepR mice are more compatible with overactivity of $\mathrm{MCH}$ neurons than exclusively of ORX. As Nts-LepR neurons are predominantly GABAergic (Leinninger et al., 2009; Goforth et al., 2014), synaptic spilling or GABA-mediated decrease of GLUergic input to $\mathrm{MCH}$ neurons may explain how these neurons modulate $\mathrm{MCH}$ neurons. As leptin serves as a metabolic signal for high energy reserves, the inhibition of $\mathrm{MCH}$ neurons by Nts-LepR neurons may be a mechanism to decrease $\mathrm{MCH}$ mediated energy conservation in states of positive energy balance.

\section{Arcuate Nucleus}

The Arc is a classical component of the feeding behavior circuitry, as lesions in this nucleus and the adjoining VMH by gold thioglucose promote hyperphagia and obesity in mice (Debons et al., 1962). This nucleus contains neuronal populations that produce anorectic peptides, such as the POMC-derived a-MSH (Jacobowitz and O'Donohue, 1978; Elias et al., 1998; Ludwig et al., 1998) and CART (Koylu et al., 1997, 1998; Kristensen et al., 1998), as well as orexigenic peptides as the NPY (Allen et al., 1983; Clark et al., 1984) and the AgRP (Ollmann et al., 1997; Shutter et al., 1997). This nucleus is also influenced by insulin (van Houten et al., 1980; Niswender et al., 2003), glucose (Leloup et al., 1994; Muroya et al., 1999; Parton et al., 2007), and leptin (Mercer et al., 1996; Schwartz et al., 1996; Cheung et al., 1997), all of them capable of conveying the short- and long-term energetic state of the organism from the CSF to the Arc through the ME (Woods et al., 1979; Banks et al., 1996), positioning the Arc as the ideal receptor for these circulating metabolic markers. 

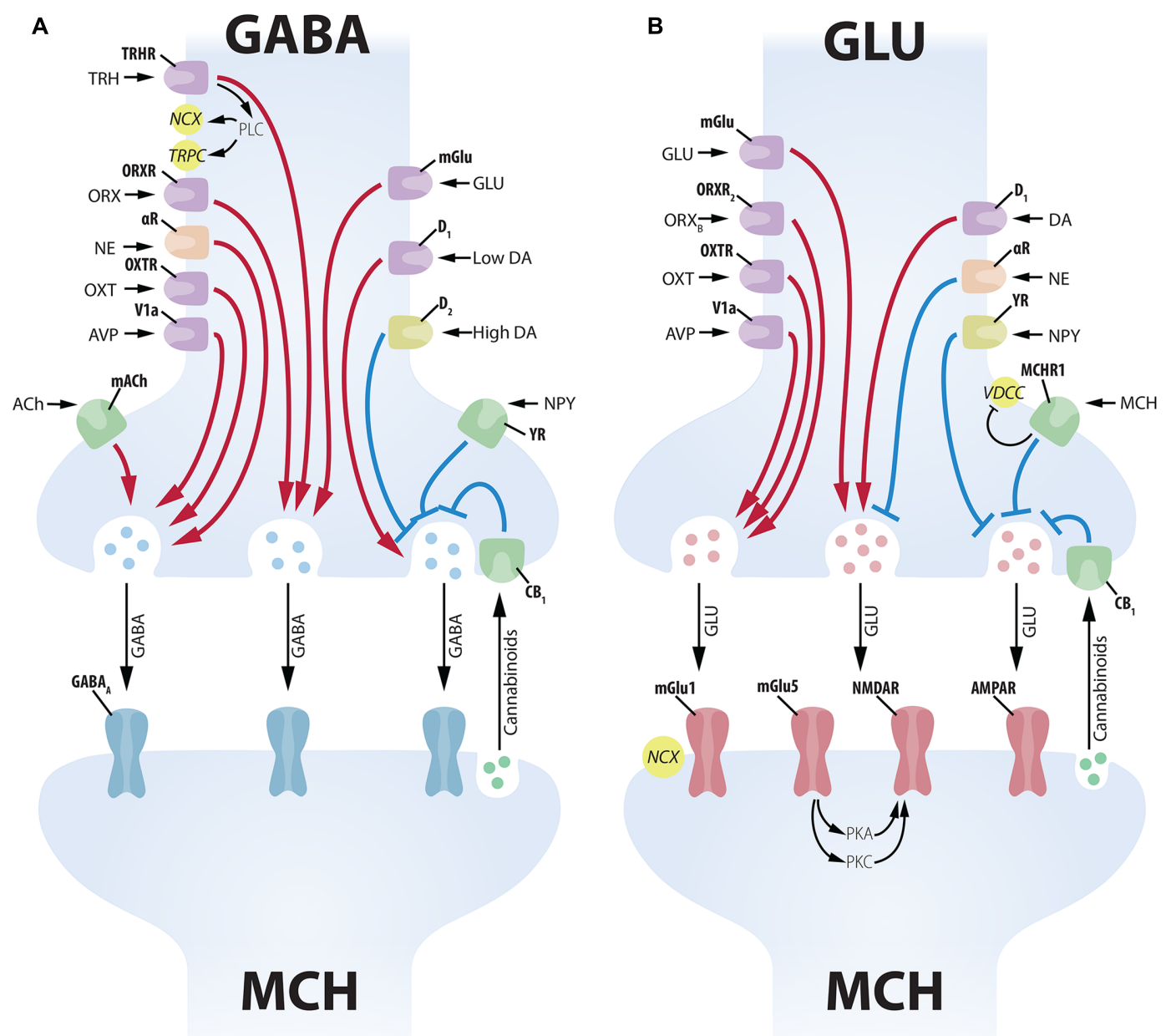

FIGURE 5 | Complex presynaptic modulation of $\mathbf{M C H}$ neurons. (A) Several neuropeptides increase GABAergic input to $\mathrm{MCH}$ neurons acting on the soma of GABA neurons, such as: TRH, ORX, NE, OXT, AVP, GLU and low levels of DA. ACh increases GABAergic input to MCH neurons acting on axon terminals. High levels of DA, on the other hand, promote an inhibition of presynaptic GABAergic input through $\mathrm{D}_{2}$ receptors. NPY decreases inhibitory input acting on presynaptic terminals, while cannabinoids released by the $\mathrm{MCH}$ postsynaptic terminal decrease vesicle release (Huang et al., 2007). GABAergic input to MCH neurons is vehiculated mainly by $\mathrm{GABA}_{\mathrm{A}}$ receptors. (B) The GLUergic input to $\mathrm{MCH}$ neurons is similarly under the control of numerous inputs. GLU, ORX, OXT, AVP, and DA act on the soma of GLU cells to increase excitatory input to MCH neurons. NE and NPY have the opposite effect, decreasing GLU input to MCH neurons. MCH has an inhibitory presynaptic effect through an MCHR1-dependent decrease in $\mathrm{Ca}^{2+}$ currents. Cannabinoid activation of $\mathrm{CB}_{1}$ has a similar inhibitory effect on vesicle release (Huang et al., 2007). GLU input to MCH neurons is conveyed by multiple receptors, including ionic (NMDAR and AMPAR) and mGlu1 and mGlu5. The activation of mGlu5 in MCH neurons leads to a potentiation of NMDAR responses through protein kinases $\mathrm{A}$ and $\mathrm{C}$ (PKA and PKC).

Elias et al. (1998) showed that both major populations of the Arc, NPY/AgRP and POMC/CART, project densely to the LHA, with immunoreactive fibers in close apposition to $\mathrm{MCH}$ neurons. van den Pol et al. (2004) report that NPY inhibits $\mathrm{MCH}$ neurons, decreasing spike frequency and hyperpolarizing membrane potential, both postsynaptically through GIRK currents and presynaptically inhibiting GLU and GABA release to $\mathrm{MCH}$ neurons (Figures $4 \mathrm{~B}, \mathbf{5}$ ). To better understand these observations, it is important to consider that the LHA and the Arc present reciprocal connections. Direct injections of $\mathrm{MCH}$ in the Arc promote an increase in food consumption, placing this area as a site of MCH action (Abbott et al., 2003). Using hypothalamic cultures, these authors demonstrated that the addition of $\mathrm{MCH}$ to the culture induces a release of NPY, at the same time decreasing the amount of the anorectic $\alpha$-MSH released by a hypothalamic explant. These data suggest that $\mathrm{MCH}$ projections from the LHA to the Arc may increase the release of the orexigenic peptide NPY and decrease the anorectic $\alpha-\mathrm{MSH}$, resulting in an overall increase in food consumption.

The results of Abbott et al. (2003) allow us to speculate that NPY inhibition of MCH neurons may be part of a negative feedback loop, with increased $\mathrm{MCH}$ activity on the Arc resulting in a depression of $\mathrm{MCH}$ neurons by NPY, avoiding excessive stimulation of this orexigenic circuit. Another possibility is that NPY-MCH interactions may be secondary to some other Arc-LHA circuits, as suggested by Segal-Lieberman et al. (2003) and van den Pol et al. (2004). Parallel to this negative feedback loop, a positive feedback loop may exist between $\mathrm{MCH}$ and 
the anorectic peptides of the Arc. Although the addition of $\alpha$-MSH agonists or antagonists has no major electrophysiological effect on MCH neurons (van den Pol et al., 2004), the selective deletion of the Pomc gene results in a marked increase in Pmch mRNA, suggesting that POMC-derived peptides are capable of inhibiting Pmch expression (Challis et al., 2004). In this case, inhibition of $\alpha$-MSH neurons by $\mathrm{MCH}$ would result in decreased inhibition of Pmch expression, potentially increasing the stimulation of NPY by MCH and reinforcing the negative feedback loop. It is important to consider, however, that these circuits may be polysynaptic, since there is little evidence for NPY and melanocortin receptors in MCH neurons (Liu et al., 2003; Parks et al., 2014). Therefore, these circuits may employ interneurons or even ORX neurons, since these are susceptible to multiple regulatory mechanisms by NPY (Fu et al., 2004). Finally, another possibility is that Arc projections interact with $\mathrm{MCH}$-ir fibers at the PVH, a target nucleus that display important effector actions regarding feeding behavior and energy homeostasis.

\section{Paraventricular Hypothalamic Nucleus}

The PVH is one of the better-described nuclei in the whole hypothalamus. It has eight major subdivisions, with three magnocellular subdivisions containing neurons that primarily synthesize OXT and AVP, and five parvocellular subdivisions containing neurons that synthesize CRF and TRH, although we now know that many other neurochemical populations are present in the PVH with distinct distributions (Swanson and Sawchenko, 1983; Simmons and Swanson, 2008, 2009). In many ways, the PVH can be regarded as the motor nucleus of the endocrine system, directly releasing OXT and AVP in the NH and $\mathrm{CRF}$ and TRH in the portal circulation to modulate $\mathrm{AH}$ activity and influence two of the major neuroendocrine axis in the brain, the HPT and the HPA axis. Projections from these neurons, however, are not restricted to the hypophysis, as they project densely to other areas of the brain and all of them show, to some extent, modulatory effects.

To investigate if OXT and AVP neurons could exert effects through $\mathrm{MCH}$ neurons, Yao et al. (2012) evaluated the electrophysiological response of $\mathrm{MCH}$ cells to these neuromodulators. AVP directly excites $\mathrm{MCH}$ neurons through the V1a receptor, depolarizing their membrane potential and increasing spike frequency through NCX and the opening of TRPC (Figure 4A). AVP also acts presynaptically increasing GLU- and GABAergic input to $\mathrm{MCH}$ neurons (Figure 5). OXT provides excitatory input to $\mathrm{MCH}$ neurons as well, through the OXTR, depolarizing their membrane potential and increasing spike frequency through NCX (Yao et al., 2012) (Figure 4A). In addition to OXT and AVP, neuropeptides from the PVHp may also contribute to $\mathrm{MCH}$-dependent modulation of ingestive behavior. As discussed in the previous section, TRH modulates $\mathrm{MCH}$ function and it is not possible, for now, to specify the exact source of this input to presynaptic GABA neurons in the LHA. Moreover, little has been described about the effects of CRF-synthesizing neurons over $\mathrm{MCH}$ cells albeit that, if such mechanism exists, it must involve polysynaptic circuits, since Parks et al. (2014) report that CRF receptors are not significantly found in $\mathrm{MCH}$ neurons.

\section{Limbic and Mesolimbic}

The Acb is an important component of the ventral striatum, participating in several processes, including reward and aversion (for a review, see Carlezon and Thomas, 2009). The Acb can be divided into two subdivisions, a core (AcbC) and a shell (AcbSh), with distinct connectivity, cellular composition and function. AcbSh activity has been associated with feeding inhibition, as electrical and optogenetic stimulation of AcbSh MSNs are capable of quickly interrupting a feeding bout; a subset of AcbSh neurons decrease their activity immediately before the onset of a sucrose solution consumption; and photoinhibition of dopamine-sensitive $\left(D_{1}\right)$ MSNs increases liquid fat intake (Krause et al., 2010; O'Connor et al., 2015).

AcbSh MSNs are GABA cells that project to the rostral parts of the LHA, suggesting the inhibition of orexinergic neurons of this area (MCH and ORX) as a putative mechanism for AcbShmediated inhibition of feeding. It is now understood, however, that AcbSh input to the LHA does not reach $\mathrm{MCH}$ or ORX neurons directly. Sano and Yokoi (2007) identified a group of GLU neurons in the anterior part of the LHA (LHAa) that receive dense projections from the AcbSh and project to the posterior part of the LHA (LHAp), suggesting that a GLU node exists between accumbal projections and neurons in the LHAp, where $\mathrm{MCH}$ neurons are located. O'Connor et al. (2015) identified a second pathway, reporting that $\mathrm{D}_{1}$-MSNs from the AcbSh project to the LHA and inhibit GABA interneurons in this area to promote accumbal inhibition of feeding. As an AcbSh|GABALHA|GABA-LHA|MCH circuit would be ultimately excitatory for $\mathrm{MCH}$ neurons, it is likely that additional nodes are present in this pathway. Further experiments will be necessary to elucidate the intra-LHA circuit that conveys accumbal information to $\mathrm{MCH}$ and ORX neurons.

The inhibition of $\mathrm{MCH}$ neurons by accumbal projections agrees well with the theory proposed by Kelley et al. (2005) that the AcbSh functions as a "sensory sentinel," quickly inhibiting a feeding bout in response to an environment cue that may need the animal to respond to, such as a predator approaching, even if the animal's metabolic state would drive him to continue feeding. Since MCH neurons project to the MCx (Elias et al., 2008) and decrease locomotor activity, an inhibition of these neurons could, at the same time, decrease the drive to feed and disinhibit the $\mathrm{MCx}$, prompting the animal to show a motor response to the environmental cue.

Amygdaloid input to $\mathrm{MCH}$ neurons may also pay a role in the inputs to this system. Nakamura et al. (2009) report that anterograde tracer injections centered in the CeA reveal numerous varicose fibers in the LHA, chiefly on its dorsolateral most area. These fibers often make contact to $\mathrm{MCH}$-ir and ORX-ir neurons and these terminals are immunoreactive for a GABA marker, suggesting an inhibitory property for these CeA projections.

\section{Midbrain and Hindbrain}

The midbrain contains some of the most important nuclei containing DA-synthesizing neurons in the whole CNS, including the A8, SN - A9, and VTA - A10 groups (Dahlström and Fuxe, 1963). These groups project densely to the forebrain, including 
the neocortex, the striatum and the diencephalon (Andén et al., 1966). Leibowitz and Brown (1980) showed that ventral A8 and A9 cells provide an important CA input to the perifornical area, and lesions of the A8 and A9 deplete the LHA of CA-ir, reduce the anorectic effect of the DA presynaptic drug amphetamine and potentiate the anorectic effect of DA itself. Alberto et al. (2011) and Conductier et al. (2011) report that DA produces hyperpolarization of $\mathrm{MCH}$ neurons through GIRK mediated outward currents, but DA receptors $\left(\mathrm{D}_{1}\right.$ and $\left.\mathrm{D}_{2}\right)$ antagonists failed to induce similar currents, suggesting that the observed DA actions are not mediated by DA receptors (Figure 4B). Conductier et al. (2011) also found that DA can alter the presynaptic input to $\mathrm{MCH}$ neurons, with low concentrations of DA $(1 \mu \mathrm{M})$ potentiating both GABA and GLU release through presynaptic $\mathrm{D}_{1}$ receptors, while high concentrations of DA $(100 \mu \mathrm{M})$ inhibits GABA release through $\mathrm{D}_{2}$ receptors, with GABA effects more pronounced than GLU effects suggesting a predominantly inhibitory action for DA over $\mathrm{MCH}$ neurons (Figure 5). Both Alberto et al. (2011) and Conductier et al. (2011) found as well that the postsynaptic effect of DA depends on the adrenergic receptor $\alpha_{2 \mathrm{~A}}$, since specific antagonists blocks DA-induced currents and $\alpha_{2 \mathrm{~A}}$ is present in $\mathrm{MCH}$ neurons (Modirrousta et al., 2005).

These results are particularly noteworthy because the $\alpha_{2 \mathrm{~A}}$ is also the receptor through which $\mathrm{NE}$ inhibits $\mathrm{MCH}$ action. The LHA is moderately innervated by NE terminals originated from the brainstem (Palkovits et al., 1974), with an important contribution from the lateral tegmental NE system (Moore and Bloom, 1979). The work of van den Pol et al. (2004) has provided evidence that NE promotes a hyperpolarization of the membrane potential and a decrease in spike frequency of active $\mathrm{MCH}$ neurons by mobilizing GIRK currents, and this effect is blocked by a selective $\alpha_{2 \mathrm{~A}}$ antagonist (Figure 4B). NE also acts on the synaptic input to $\mathrm{MCH}$ neurons, decreasing GLUergic and increasing GABAergic input, resulting in an overall inhibitory action (Figure 5). Taken together, the results of van den Pol et al. (2004), Alberto et al. (2011), and Conductier et al. (2011) point to a synergistic convergence of both DA and NE systems through the $\alpha_{2 A}$. This convergence may limit how much synergistic inhibition acts over MCH neurons, since DA inhibition would desensitize the receptors for NE inhibition and vice-versa. The DA and NE systems are, therefore, competitive elements instead of synergistic, even though both have inhibitory properties over $\mathrm{MCH}$ neurons.

Serotonin is a monoamine neurotransmitter synthesized in numerous groups of neurons of the brainstem and with widespread projections throughout the CNS (Dahlström and Fuxe, 1963; Steinbusch, 1981). Hypothalamic injections of 5-HT have been extensively correlated to a decrease in feeding (for a review, see Leibowitz and Alexander, 1998) through multiple receptors and different mechanisms (for a review, see De Vry and Schreiber, 2000). van den Pol et al. (2004) report that 5-HT hyperpolarizes $\mathrm{MCH}$ neurons and decreases spike frequency through postsynaptic mechanisms (Figure 4B). Taken into consideration, these data indicate that 5-HT may decrease feeding through the inhibition of $\mathrm{MCH}$ neurons in addition to 5-HT interactions with DA, although further experimental work is necessary to confirm this hypothesis and detect the specific 5-HT receptor subtypes responsible for this action.

The urocortins (UCN1, UCN2, and UCN3) are members of the mammalian CRF family, with a special affinity for the CRF receptor subtype 2 (Vaughan et al., 1995; Lewis et al., 2001; Reyes et al., 2001). UCN1 has been mapped in the albino rat by Kozicz et al. (1998) and Bittencourt et al. (1999), who have shown that the main sites of Ucn1 mRNA expression and UCN1 synthesis are the EW and the superior olivary nucleus, both brainstem structures, with a broad distribution of UCN1-ir fibers throughout the CNS. Although the EW was initially recognized as a motor nucleus for vision related muscles, it is now recognized that the EW has two components: a preganglionic $\mathrm{EW}$, responsible for this cholinergic motor control, and the centrally-projecting EW, involved in a series of functions such as feeding, stress and addiction (for a review in EW structure and function, we point the reader to Kozicz et al., 2011). Of special importance to us is the role of UCN1 in feeding behavior, as UCN1 acts as a powerful anorectic peptide in nanomolar concentrations (Spina et al., 1996) and diet/fasting can regulate Ucn1 mRNA expression (Legendre et al., 2007; Xu et al., 2009). Júnior et al. (2015) have shown that UCN1-ir neurons or the EWcp send projections to the LHA and these fibers are in close proximity to $\mathrm{MCH}$-ir neurons, so this UCN1|EWcp$\mathrm{MCH} \mid \mathrm{LHA}$ circuit could represent the pathway through which UCN1 influences feeding behavior. Although the low levels of CRF receptors in $\mathrm{MCH}$ could be taken as an evidence against this particular pathway, it is noteworthy that these receptors are found on the LHA (Chalmers et al., 1995), so the action of UCN1 could involve the modulation of GABAergic or GLUergic interneuronal input to $\mathrm{MCH}$ neurons. Further studies will be necessary to elucidate the physiological aspects of this circuit.

\section{Circulating Factors}

The $\mathrm{MCH}$ peptidergic system appears to be heavily influenced by leptin, a major circulating factor of satiety produced by adipocytes (Zhang et al., 1994; Campfield et al., 1995; Halaas et al., 1995; Pelleymounter et al., 1995). Qu et al. (1996) were the first to identify a relationship between $\mathrm{MCH}$ and leptin, reporting that $L e p^{\mathrm{ob} / \mathrm{ob}}$ mice have twice the level of Pmch mRNA than WT animals, suggesting a leptin inhibition of Pmch expression. Agreeing to that, obese rats with a point mutation in the long form of the LepR gene (Zucker rats $L e p R^{\mathrm{fa} / \mathrm{fa}}$ ) display higher levels of Pmch mRNA and decreased levels of Mchr1 mRNA in comparison to lean rats, suggesting that deficient leptin signaling can upregulate the $\mathrm{MCH}$ system (Stricker-Krongrad et al., 2001). Likewise, Gavrila et al. (2005), studying humans, report that fasting (with a concomitant fall of circulating leptin levels) significantly increases serum levels of $\mathrm{MCH}$, and supplementation with $\mathrm{r}$-metHuLep prevents the raise of seric $\mathrm{MCH}$. The mechanism through which $\mathrm{MCH}$ responds to leptin, however, must be polysynaptic, as studies using a reporter associated to the Lepr gene indicate that Lepr-expressing neurons are not MCH or ORX (Leinninger et al., 2009; Louis et al., 2010).

Other important factors for metabolism regulation are glucose and insulin. Although the periphery holds the machinery for glucose-mediated insulin production, and this machinery works 
efficiently to keep the blood levels of glucose in a strict range, we now include the CNS as an important sensor for these metabolic cues and as an effector to keep their physiological levels (Myers and Olson, 2012). The IR is widely distributed in the CNS (Mayer et al., 1978), with deleterious effects on energy homeostasis occurring after its specific deletion (Brüning et al., 2000). Hausen et al. (2016) provide evidence that $\mathrm{MCH}$ neurons can respond to insulin, reporting that $200 \mathrm{nM}$ of it is capable of activating PIP3-dependent pathways and invoking action potentials in 6 of 13 neurons tested, while 3 neurons displayed a decrease in firing and the remaining neurons showed no alteration, suggesting that subpopulations of $\mathrm{MCH}$ neurons are responsive to insulin with different dynamics (Figure 4A).

In addition to insulin, Burdakov et al. (2005) provided evidence that glucose is also able to modulate $\mathrm{MCH}$ neurons. Although not as high as plasma levels, the brain is subjected to variations in glucose concentration that mirror those of the plasma, with a physiological range that goes from $\sim 0.7-2.5 \mathrm{mM}$ and may reach as low as $0.2 \mathrm{mM}$ in conditions of hypoglycemia and $5 \mathrm{mM}$ in hyperglycemia (Routh, 2002). A subset of approximately $70 \%$ of $\mathrm{MCH}$-ir neurons is excited by high levels of glucose $(5 \mathrm{mM})$ and dose-dependently hyperpolarizes as glucose levels reach 0.2 mM (Burdakov et al., 2005; Kong et al., 2010). Kong et al. (2010) demonstrated that glucose sensing by $\mathrm{MCH}$ neurons works in a similar fashion to pancreatic $\beta$-cells, in which an increase in intracellular glucose (driven by an increase in extracellular glucose carried by the GLUT3 transporter) results in increased ATP production which, in turn, induces the closure of $\mathrm{K}_{\mathrm{ATP}}$ with consequent depolarization, and this effect is dependent of SUR1-containing $\mathrm{K}_{\mathrm{ATP}}$, establishing the SUR1 protein as a marker for glucose-sensitive MCH neurons (Figure 4A).

Although the activation of $\mathrm{MCH}$ in conditions of high glucose may seem counterintuitive, it is possible to fit it in a larger role for $\mathrm{MCH}$ in energy homeostasis. $\mathrm{MCH}$ is suggested to be involved in energy conservation, decreasing locomotor activity and metabolic parameters and increasing sleep in conditions of positive energy balance, thus its activation in response to high glucose (Burdakov et al., 2013). This led us to hypothesize that the orexigenic activity of $\mathrm{MCH}$ is not incompatible with that suggestion, when we consider the probable environment of an animal regarding food availability. It would be dangerous for animals to wait for their energy levels get low, as this could impair their capacity to obtain food, so it is beneficial for animals to eat periodically, even when their energy balance is positive. In most situations, animals have some awareness of their surroundings, and sources of food probably have been scouted, with animals preferentially occupying areas with abundant food availability. In that case, animals do not have to explore for food, so they may display decreased locomotor activity and longer periods of rest. Still, they must have some drive to feed periodically, and $\mathrm{MCH}$ could account for this effect, integrating not only glucose levels but also the other input discussed in this section. Food depletion of their environment or hazards (such as predators) may impair their ability to keep this constant state, in a situation that would lead to decreased levels of glucose. In this case, $\mathrm{MCH}$ would need to be shut off (low-glucose induced hyperpolarization) and a system that promotes food-seeking could be activated.
A possible component of this food circuit is ORX, as ORX-ir cells are excited by low levels of glucose (Burdakov et al., 2005) and their activation is also implicated in arousal (Alexandre et al., 2013), a necessary condition for active food-seeking. In this model, these two peptides could act in different aspects of feeding behavior: $\mathrm{MCH}$ is responsible for keeping a positive energy-balance state, while the ORX system "kicks in" when the animal reaches a negative energy balance and must return to a safer range. Since $\mathrm{MCH}$ is a "baseline stabilizer," avoiding big fluctuations in energy homeostasis, $\mathrm{MCH}$ inactivation would not promote big alterations in feeding, since other ingestivecontrolling systems would act nevertheless when the animal entered deficient energy states, possibly consuming an amount of food similar to that of an MCH-preserved animal. This appears to be the case, since the selective ablation of $\mathrm{MCH}$-neurons in adult mice by diphtheria toxin promotes leanness and hyperactivity, but the animals display only a non-significant tendency to eat less than control mice (Whiddon and Palmiter, 2013).

Another possible peripheral signal that may influence $\mathrm{MCH}$ neurons is CORT, a glucocorticoid produced by the adrenal gland that freely crosses the $\mathrm{BBB}$ to influence the regulation of energy balance, with central and systemic administration of glucocorticoids promoting an increase in food intake and body weight (Freedman et al., 1986; Green et al., 1992; Tataranni et al., 1996; Zakrzewska et al., 1999). Drazen et al. (2004) have shown that, in ADX animals, the icv injection of $\mathrm{MCH}$ has a less potent effect than in sham animals regarding both food and fluid intake, and this effect is reversed in ADX animals that receive CORT supplementation in their water. These authors also report that hypothalamic expression of Pmch mRNA is decreased in ADX animals. This is in good agreement with the results of Viale et al. (1997) that showed the partial sequence of a glucocorticoid response element in the $5^{\prime}$-flanking region of the Pmch gene, which could account for the mechanism through which the absence of CORT after ADX promotes a decrease in Pmch mRNA expression.

Amylin is a pancreatic peptide hormone, co-synthesized with insulin by $\beta$-cells in response to feeding (Leffert et al., 1989), although central AMY has been described as a lactation-related peptide synthesized in the MPOA (Dobolyi, 2009). AMY has an anorectic action, suppressing feeding in both rats and mice, suggesting a post-prandial satiety effect and long-term lipostatic signaling (Lutz et al., 2001; Rushing, 2003). The intraperitoneal injection in rats of salmon calcitonin, an AMY receptor agonist, decreases the levels of LHA Pmch mRNA (Barth et al., 2003). This effect is probably not direct, since there is little evidence for AMY binding in the LHA (Sexton et al., 1994), suggesting that AMY signaling reaches $\mathrm{MCH}$ neurons through circuits that may include the AP (Lutz et al., 2001), VTA (Mietlicki-Baase et al., 2013, 2015) and LDTg (Reiner et al., 2017).

\section{Outputs}

The regulation of feeding by $\mathrm{MCH}$ neurons is a complex mechanism that involves many aspects of this behavior, including the energy balance of the organism, metabolic rate, rewarding circuits, locomotor activity, arousal, spatial memory, olfactory cues and complex interactions with other peptidergic systems. In 
this section, we will highlight the main actions of $\mathrm{MCH}$ and its underlying connectivity, illustrated in Figure 2B.

\section{Locomotor Activity}

$\mathrm{MCH}$-ir neurons project diffusely to numerous areas of the isocortex, including the SSCx, MCx, infralimbic, and Ins, as shown by Saper et al. (1986) using an antibody for $\alpha$-MSH that was, as we now know, cross-reacting to $\mathrm{MCH}$. This pattern of $\mathrm{MCH}$ projections was confirmed by Cvetkovic et al. (2003) using an antibody for salmonid $\mathrm{MCH}$ and further explored by Elias et al. (2008), that showed extensive innervation of the MCx by $\mathrm{MCH}$ neurons of the LHA. These authors also reported that a subset of these $\mathrm{MCH}$-ir $\mathrm{MCx}$-innervating neurons also innervate the PPTg, what may also contribute to a motor regulation through the mesencephalic locomotor region of this area. This circuit supports an action of $\mathrm{MCH}$ decreasing locomotor activity as reported in $\mathrm{Pmch}^{-/-}$and $\mathrm{Mchr}^{-/-}$mice (Segal-Lieberman et al., 2003; Willie et al., 2008), as well as progressive (Alon and Friedman, 2006) and sudden (Whiddon and Palmiter, 2013) adult ablation of $\mathrm{MCH}$ neurons. Through this pathway, $\mathrm{MCH}$ neurons decrease locomotor activity and energy expenditure to revert states of negative energy balance or sustain states of positive energy balance.

\section{Energy Expenditure}

Locomotor activity is not the only way the CNS can modulate energy expenditure, since thermogenesis also play an important role in this aspect of homeostatic balance. The first link between $\mathrm{MCH}$ and energy expenditure control was drawn by Shimada et al. (1998), that noticed a reduction in body weight that surpassed the reduction in food intake in $\mathrm{Pmch}^{-/-}$animals. Although these animals did not have a lower rectal temperature when compared to WT mice, their rate of $\mathrm{O}_{2}$ consumption normalized for body weight was $20 \%$ higher. Mice lacking MCHR1 also display higher metabolic rates during the dark phase as measured by indirect calorimetry (Marsh et al., 2002).

Using a model of cold exposure, Pereira-da-Silva et al. (2003) showed that MCH is increased by almost $60 \%$ in coldexposed animals. These animals display a series of physiological adaptations to the challenging temperature that include an increase in food consumption, fall of body weight and thermogenesis. These authors report that cold-exposed animals with impaired $\mathrm{MCH}$ synthesis have an increase in the molecular machinery necessary for BAT-dependent thermogenesis when compared to control animals, suggesting that $\mathrm{MCH}$ inhibits BAT thermogenic activity, potentially conserving energy for homeostatic purposes. To investigate the chemical signature of the hypothalamic circuits modulating BAT activity, Oldfield et al. (2002) injected a polysynaptic viral tracer in the BAT of adult rats combined to immunohistochemistry for different neuropeptides synthesized in the hypothalamus. Third-order $\mathrm{MCH}$-ir neurons were found in the LHA, suggesting that $\mathrm{MCH}$ neurons provide input to the BAT through brainstem nuclei.

These results are reinforced by the works of Guesdon et al. (2009) and Chung et al. (2010). Guesdon et al. (2009) report that intraventricular injections of $\mathrm{MCH}$ decrease lipid oxidation and Chung et al. (2010) demonstrated that $\mathrm{Mchrl}^{-/-}$animals are resistant to weight gain when exposed to HFD due increased lipid metabolism. Furthermore, Verty et al. (2010), using temperaturesensitive telemetry probes, registered a significant increase in BAT temperature after intraventricular injection of an MCHR1 antagonist. All these data suggest that LHA MCH neurons polysynaptically decrease thermogenesis in BAT to reduce lipid metabolism and energy expenditure, an important mechanism to sustain a positive energy balance or revert a scenario of negative energy balance.

\section{Autonomic Modulation}

Saper et al. (1976) have shown that LHA neurons project both to autonomic centers of the brainstem, such as the dorsal motor nucleus of the vagus and the NTS, and directly to the $\mathrm{SpCd}$, including the ImC in thoracic levels, suggesting a descending hypothalamo-autonomic pathway that access directly the preganglionic cells of the parasympathetic and sympathetic systems. Since MCH-ir fibers from the LHA also sparsely innervate the autonomic nuclei of the brainstem and all levels of the SpCd (Kohler et al., 1984; Bittencourt et al., 1992; Bittencourt and Elias, 1998), this represents a possible pathway through which $\mathrm{MCH}$ may integrate ingestive behavior to autonomic function, although further evidence is necessary to establish if there is a direct contact between ganglionic cells and $\mathrm{MCH}$ neurons, possibly through specific viral tracers. More recently, Pérez et al. (2011) injected a polysynaptic viral tracer in the SAL and MAS of mice and discovered that $\mathrm{MCH}$-ir neurons of the LHA provide inputs to brainstem nuclei involved in the control of these structures (MoV - MAS innervation; 7N and SuS - SAL innervation). Through this innervation, LHA MCH-containing neurons can gate two important preparatory responses to feeding: salivation, an autonomic response necessary for bolus formation and swallowing, and mastication, a motor response which involves complex coordination between muscles of the jaw, face, and tongue. Another interesting aspect of this $\mathrm{MCH}$ input is the fact that both salivation and mastication are under the control of higher-order cortical centers (Thexton, 1992; Hubschle et al., 1998), and MCH could potentially integrate this highercenter input and provide the output to lower brainstem nuclei.

An indirect mechanism for this feeding-autonomic coupling is through the PVH. An important number of labeled cells are found in the PVH after retrograde tracer injection in the SpCd, suggesting that this nucleus also acts over autonomic systems (Hancock, 1976; Saper et al., 1976; Ono et al., 1978; Blessing and Chalmers, 1979; Hosoya and Matsushita, 1979). The PVH neurons that project to the $\mathrm{SpCd}$ and to the dorsal vagal complex are concentrated in the dorsal, lateral, and medial parts of the PVHp (Swanson and Kuypers, 1980), regions that receive more MCH-ir innervation than the PVHm (Bittencourt et al., 1992). Since injections of $\mathrm{MCH}$ restricted to the $\mathrm{PVH}$ without spilling to the ventricular system promote an increase in feeding behavior (Abbott et al., 2003), this could represent another node through which $\mathrm{MCH}$ integrates feeding behavior and autonomic function, 
triggering the necessary autonomic preparatory processes to feeding.

\section{Thyroid Modulation}

Kennedy et al. (2001) suggest that MCH could be part of the peptidergic modulation of the HPT axis. After icv injections of $\mathrm{MCH}$, there is an in vivo decrease in the plasmatic levels of TSH (10 and $60 \mathrm{~min}$ after injection). In vitro, the addition of $\mathrm{MCH}$ and $\mathrm{NEI}$ in hypothalamic cultures promotes a decrease in the levels of TRH, and MCH co-applied with TRH decreases the secretion of TSH in cultures of hypophysaire cells when compared to TRH alone. MCH may act both through a direct and an indirect pathway to control the HPT axis activity. Directly, MCH-ir fibers from the LHA could innervate TRH-ir neurons in the $\mathrm{PVH}$, decreasing their secretory activity. In the indirect pathway, MCH-ir projections to the Arc could potentially act over the $\alpha$-MSH/CART and NPY/AgRP systems to promote downstream alterations in TRH synthesis. There is anatomical support for both pathways. Both MCH-ir fibers and MCHR1 immunoreactivity are found in the PVH of rats (Bittencourt et al., 1992; Hervieu et al., 2000; Saito et al., 2001) and a reporter to associated to Mchrl can be detected in TRH-ir cells of the PVH (Chee et al., 2013). An Arc mediated pathway is supported by the observation that the neonatal ablation of the Arc abolishes the decrease in PVH| TRH resulting from fasting (Legradi et al., 1998). NPY-ir and AgRP-ir nerve terminals contact TRH-ir neurons in the $\mathrm{PVH}$, and the main source for this innervation is the Arc (Legradi and Lechan, 1998, 1999). A similar innervation of pro-TRH mRNA expressing-neurons is also observed for $\alpha$-MSH and CART fibers (Fekete et al., 2000). Regardless of the pathway, considering the energy conservation effects of $\mathrm{MCH}$, it is likely that this peptide acts on the HPT axis to decrease energy expenditure.

\section{Circulation}

There are few studies that examined the levels of $\mathrm{MCH}$ in the bloodstream. Evaluating the possibility that $\mathrm{MCH}$ was produced and released by adipocytes, Bradley et al. (2000) did not detect Pmch mRNA in these cells, but they detected $\mathrm{MCH}$ in the plasma through a RIA. Stricker-Krongrad et al. (2001) evaluated the amount of MCH in the serum of Zucker rats and found that $\mathrm{MCH}$ levels were increased in the obese animals compared to the lean ones. On the other hand, in Wistar rats with obesity resulting from electrolytic $\mathrm{VMH}$ or $\mathrm{PVH}$ lesions, there was a decrease in the intensity of MCH staining in the LHA (9.3\% for $\mathrm{VMH}$ lesions and $21.9 \%$ for PVH lesions), but the decrease in serum MCH levels did not reach statistical significance (Sun et al., 2004). It is important to consider, however, that Sun et al. (2004) used densitometry in LHA slices after immunohistochemistry to quantify the alterations in $\mathrm{MCH}$ immunofluorescence, a method that allows the detection of general differences but renders impossible to define if subsets of neurons had alterations. Therefore, it is still possible that some neurons displayed a decrease in $\mathrm{MCH}$ synthesis in response to the lesions, but the neurosecretory neurons kept stable levels of synthesis.

Gavrila et al. (2005) examined serum levels of $\mathrm{MCH}$ in humans, evaluating correlation factors for diverse parameters such body fat, leptin, estrogen and testosterone, dietary patterns and fasting. These authors found a direct correlation between serum $\mathrm{MCH}$ and fat mass and fat percentile, but no correlation could be drawn between $\mathrm{MCH}$ levels and estrogen, testosterone or dietary parameters. Working also with humans, Carnier et al. (2010) evaluated obese adolescents with eating disorders after 6 months and 1 year of interdisciplinary therapy, and observed a short-term increase of $\mathrm{MCH}$ in the blood of these patients followed by a decrease after 1 year of therapy. These authors suggest that the short-term increase in energy demand upregulate the release of $\mathrm{MCH}$ and the long-term correction of dietary habits promote a lower $\mathrm{MCH}$ baseline. During the preparation of this review, a new study about circulating $\mathrm{MCH}$ in humans was published by Naufahu et al. (2017). In this study, a reliable RIA was developed and tested in over 200 humans of both sexes and different body fat profiles, revealing a plasma $\mathrm{MCH}$ baseline of $19.5-55.4 \mathrm{pg} / \mathrm{ml}$ and some differential regulation of $\mathrm{MCH}$ that depends on both gender and adiposity, although further studies are necessary to fully elucidate how that regulation occurs.

The data regarding circulating $\mathrm{MCH}$ must be examined with caution. One major caveat is that Waters and Krause (2005), in a letter to the editor addressing the work of Gavrila et al. (2005), report that the commercial RIA used in most of these studies for $\mathrm{MCH}$ has non-specific reactivity with serum elements, since samples from $\mathrm{Pmch}^{-/-}$mice resulted in a positive signal. However, it is now hard to dispute that $\mathrm{MCH}$ is found in physiological levels in the bloodstream after the experiments ran by Naufahu et al. (2017). Another important aspect about circulating $\mathrm{MCH}$ is that its source is still unknown. Several peripheral tissues synthesize MCH (Hervieu and Nahon, 1995), and the role of this synthesis is, in many cases, still not associated with specific functions. Viale et al. (1997) showed that peripheral $\mathrm{MCH}$ has a different structure when compared to central $\mathrm{MCH}$, as it is processed differently and its sequence combines central $\mathrm{MCH}$ and NEI. Since many peripheral tissues display MCHR1 mRNA (Saito et al., 1999; Hill et al., 2001), it is highly likely that $\mathrm{MCH}$ released in the bloodstream from one or more sources can reach those tissues and play modulatory roles. The study of $\mathrm{MCH}$ as a secreted factor in the bloodstream represents, therefore, a prolific field of MCH study.

\section{Reward}

In an oversimplification, feeding behavior is stimulated by two main drives: a metabolic drive, guided by homeostatic cues aiming to keep the energy balance of the animal, and a reward drive that involves complex emotional aspects and is directly linked to the subjective experience of pleasure. The reward aspect of feeding is a major component of that behavior, playing a preeminent role in our modern society. Rats are willing to expose themselves to aversive stimuli, such as foot-shock, painful heat and intense cold to obtain palatable food, such as candies and soda (Cabanac and Johnson, 1983; Foo and Mason, 2005; Oswald et al., 2011). As the complex relationship between metabolic and reward aspects has been thoroughly examined by others (Saper et al., 2002; Berthoud, 2011; Ferrario et al., 2016), in this review, we will focus on the role that $\mathrm{MCH}$ may play coordinating these different drivers. 
The motivation and reward system involves a complex circuit with many relevant nodes, including the prefrontal cortex, striatum/Acb and the VTA. One of the regions with highest Mchr1 mRNA expression is the Acb (Hervieu et al., 2000; Saito et al., 2001) and $\mathrm{MCH}$ fibers are found in this area (Bittencourt et al., 1992), especially numerous in the septal pole of the AcbSh (corresponding to the dorsocaudomedial AcbSh) (Haemmerle et al., 2015). Haemmerle et al. (2015) also report that accumbal $\mathrm{MCH}$-innervation originates from neurons in all major hypothalamic areas containing $\mathrm{MCH}$-ir neurons, with the highest rate of neurons simultaneously labeled for $\mathrm{MCH}$ and retrograde tracer found in the IHy. These results are in good agreement to those of Kampe et al. (2009) that report AcbSh-projecting $\mathrm{MCH}$ neurons in the lateral hypothalamus that also project to the $\mathrm{CgCx} / \mathrm{Ins}$.

$\mathrm{MCH}$ influences feeding behavior through the AcbSh, as $\mathrm{MCH}$ injected in this area promotes an increase in chow consumption, while an MCHR1 antagonist injected in this area decreases feeding (Georgescu et al., 2005). These authors also report that MCHR1 co-localizes to enkephalin and Dyn in the AcbSh, suggesting that $\mathrm{MCH}$ may influence the opioid system through these cells, a possible crossroad between $\mathrm{MCH}$ and the reward system (Holtzman, 1974; Frenk and Rogers, 1979). These results are supported by the work of Mul et al. (2011) that, using the only published model of $\mathrm{Pmch}^{-/-}$rat, found that the $\mathrm{MCH}$ signal on the AcbSh is important to keep the motivational aspects of the feeding behavior. Therefore, this hodological aspect between the LHA, the AcbSh and limbic areas is fundamental for the characteristic control of energy balance and to increase motivational or incentive-related aspects of food consumption.

A new facet of $\mathrm{MCH}$ actions on reward was provided by Karlsson et al. (2012). Using an MCHR1 antagonist combined with self-administration of sucrose (caloric and sweet) or saccharin (just sweet), these authors found that antagonist administration reduces sucrose self-administration, but not saccharin-reinforced lever-pressing, indicating that $\mathrm{MCH}$ conveys rewarding signals from the caloric content of consumed food. The antagonist administration also decreased cue-induced reinstatement of sucrose seeking, confirming that $\mathrm{MCH}$ participates in the rewarding properties of sucrose.

Domingos et al. (2013) extended these result employing optogenetic manipulation of $\mathrm{MCH}$ neurons in mice. Photostimulation of $\mathrm{MCH}$ neurons is able to revert the innate preference of mice for sucrose over saccharin and stimulate DA release in the striatum. A similar effect is observed in sweet-blind mice, suggesting that taste is not necessary for $\mathrm{MCH}$-dependent preference for caloric ingestion. Contributing to these effects may be an activation of DA neurons in the VTA, as MCH ablation reduced FOS synthesis in this area. The VTA is densely innervated by MCH (Bittencourt et al., 1992) and MCHR1 is found in low (Saito et al., 2001; Pissios et al., 2008) to moderate (Hervieu et al., 2000) numbers in this area. CART/MCH terminals are found in the VTA, and CART-ir fibers contact DA neurons, suggesting that $\mathrm{MCH}$ and CART may work in tandem to modulate DA release by VTA neurons (Dallvechia-Adams et al., 2002), although electrophysiological data suggests otherwise (Korotkova et al., 2003). One possible explanation is that CART and $\mathrm{MCH}$ may not act directly on DA neurons, but modulate their auto inhibition. CART impairs DA-D 2 binding when co-applied with stimulatory substances (Jaworski et al., 2003; Kim et al., 2003; Moffett et al., 2011), and DA neurons are auto inhibited through $\mathrm{D}_{2}$ (Johnson and North, 1992; Momiyama et al., 1993), suggesting that CART may decrease DA-dependent inhibition of DA neurons, increasing their activity. Since $\mathrm{MCH}$ can depress the presynaptic machinery of release (Gao and van den Pol, 2001), MCH released with CART in the synapse could depress further CART release, preventing too much disinhibition of DA neurons. A similar mechanism to this has an experimental basis in the work of Yang and Shieh (2005), on which MCH impaired CART stimulation of DA activity in the Acb. Further experimental evidence, however, is necessary to test if this is the mechanism of action for $\mathrm{MCH}$, CART, and DA in the VTA.

The interactions between $\mathrm{MCH}$ and DA are not limited to the VTA, however, as these two may also interact directly on the Acb. When Chung et al. (2009) applied $\mathrm{MCH}$ or $\mathrm{D}_{1}$ and $\mathrm{D}_{2}$ agonists separately in the AcbSh, there was no change in animal behavior, but when the three were co-applied there was a potentiation of the cocaine rewarding response. Furthermore, $\mathrm{Mchr}^{-/-}$animals displayed resistance to the rewarding effects of cocaine, in a similar way to the pharmacological blockade of this receptor. More work will be necessary to fully elucidate the interaction between MCH and DA, however, as Smith et al. (2005) observed upregulation of $\mathrm{D}_{1}$ and $\mathrm{D}_{2}$ in the AcbSh of $M c h r 1^{-/-}$mice and hyper sensibility to amphetamine, while methamphetamine responses were attenuated after injections in the AcbSh of $\mathrm{MCH}$ and amplified after MCHR1 agonists injections (Sun et al., 2013). Albeit Chung et al. (2009) suggest that these apparent contradictions can be explained by the different mechanisms of cocaine and amphetamine action, more evidence is necessary to confirm this and to allow us to understand the role that $\mathrm{MCH}$ may play in reward and addiction.

As a final consideration about $\mathrm{MCH}$ and reward, although alcohol consumption is not the theme of this review, both drug and food consumption have a common ground that is the reward motivation. Alcohol is a particularly interesting model, as it is both a stimulatory factor of the reward system and a source of caloric nutrient. Duncan et al. (2005) found an increase in 10\% alcohol and isocaloric sucrose solutions intake after $3 \mathrm{~V} \mathrm{MCH}$ injections, suggesting that $\mathrm{MCH}$ may drive the pursuit of these substances for their rewarding or caloric value. Morganstern et al. (2010a) developed on these results, demonstrating that injections of $\mathrm{MCH}$ restricted to the $\mathrm{PVH}$ and $\mathrm{Acb}$ were able to increase alcohol consumption, suggesting those nuclei as downstream targets of $\mathrm{MCH}$ action on alcohol consumption. The exact mechanism by which this MCH modulation happens was, however, contentious, as some reports suggested that MCHR1 was not involved in the alcohol consumption-promoting effect of MCH (Duncan et al., 2006, 2007), while others found a potent decrease in alcohol consumption and reinstatement after an MCHR1 antagonist treatment (Cippitelli et al., 2010). Recently, Karlsson et al. (2016) reported that $\mathrm{MCH}$ and MCHR1 have a dual role in the regulation of alcohol intake through mechanisms related both to caloric intake and reward 
motivation. Further experiments on $\mathrm{MCH}$ alterations linked to alcohol consumption, however, are still necessary to provide an overarching explanation for all results reported in the literature. Understanding the mechanisms through which $\mathrm{MCH}$ modulates alcohol consumption may provide insightful views on addictive and excessive behaviors.

\section{Foraging/Predation}

The participation of $\mathrm{MCH}$ in the pre-ingestive steps of feeding is perhaps its most elusive aspect so far. Albeit the ad libitum diet used in most experimental designs is convenient to standardize parameters, it also creates a highly artificial setup for the animal. In its natural environment, the animal must constantly make decisions regarding food acquisition while factoring elements such as its current energetic availability compared to other needs, foreseen energetic necessity, proximity to food sources and the risk of being eaten by a predator or injured by another environmental hazard. Some animals must also decide when to hunt for prey, since predation may reward it with metabolic needs, if successful, but may also incur in wasted energy if a failure.

Among the many complex elements of foraging and predation, there are three aspects to which $\mathrm{MCH}$ may be associated to: sensory integration, decision-making, and memory. The first role proposed for $\mathrm{MCH}$ in the mammalian brain was over the modulation of auditory stimuli, by Miller et al. (1993). Using an auditory gating paradigm, these authors have shown that the suppression of a redundant auditory signal is abolished after $\mathrm{MCH}$ addition to the CSF, suggesting that $\mathrm{MCH}$ can induce a state of higher vigilance at the cost of decreased focus on a single signal. Besides auditory stimuli, Adams et al. (2011) provided evidence that $\mathrm{MCH}$ participates in the integration of olfactory stimuli through numerous $\mathrm{MCH}$-ir fibers that are found in olfactory-linked structures such as the Pir and the OB. Using $\mathrm{Pmch}^{-1-}$ mice, these researchers observed a decreased capacity for them to find food using olfactory cues, suggesting that $\mathrm{MCH}$ may display an important role in the search for food. $\mathrm{MCH}$ is, therefore, an important actor in the gating of sensorial information, altering perceptual properties of the animal (such as vigilance and focus) depending on the available energy stores.

Regarding decision-making, there is a paucity of functional information relating it to $\mathrm{MCH}$. Projections arising from $\mathrm{MCH}$ neurons in the IHy and tuberal LHA densely and diffusely reach the PAG (Elias and Bittencourt, 1997). Comoli et al. (2003) have shown that the lateral aspects of the PAG are involved in predatory behavior, as FOS synthesis in this area is increased in rats after cockroach predation compared to control animals. It is possible, therefore, that $\mathrm{MCH}$-ir projections to the PAG may influence predatory behavior, but experimental evidence is necessary to establish the PAG-mediated actions of $\mathrm{MCH}$.

The third important aspect of foraging is memory, as animals that can adequately remember the location of food sources will have a survival advantage. The HF has been thoroughly implicated in memory (Scoville and Milner, 1957; Zola-Morgan et al., 1986; Squire, 1992) and is one of the major targets of
$\mathrm{MCH}$-ir fibers and MCHR1 synthesis in the rat brain (Bittencourt et al., 1992; Hervieu et al., 2000; Saito et al., 2001). Lima et al. (2013) provided a more detailed description of the $\mathrm{MCH}$ input to the HF, describing a higher density of $\mathrm{MCH}$-ir fibers in the dorsal HF, especially in the $\mathrm{CA} 3$ field, and the presence of $\mathrm{MCH}-$ ir fibers plexuses around GABAergic basket cells in CA1 and $\mathrm{CA} 3$, in addition to apparent contacts between $\mathrm{MCH}$-ir fibers and HF-projecting cholinergic cells of the MS.

Several authors have provided evidence that $\mathrm{MCH}$ plays a role in $\mathrm{HF}$ modulation. After $\mathrm{CA} 1 \mathrm{MCH}$ injections, rats show: improved acquisition and consolidation in an inhibitory avoidance test (Monzon et al., 1999); a decrease in the levels of nitric oxide and its second messenger cGMP (Varas et al., 2002a); and a facilitation in long-term potentiation through alterations in the NMDA receptor-gated channel (Varas et al., 2002b, 2003). Adamantidis et al. (2005) provided further evidence for these memory-facilitation actions of $\mathrm{MCH}$ by reporting that $M c h 1^{-/-}$mice have worse memory retention of aversive stimuli and decreased response of pyramidal cells to NMDA in the CA1. Pachoud et al. (2010) expanded the roles of $\mathrm{MCH}$ in HF plasticity, reporting that $M c h r 1^{-/-}$mice have decreased output from excitatory GLUergic Schaffer Collaterals from the CA3 to the CA1 through both AMPA- and NMDA-mediated responses. In summary, these results point to $\mathrm{MCH}$ influencing GLUergic transmission in the CA1 field through both presynaptic mechanisms and the increase of NMDAR and AMPAR.

While most studies focused on the effects of $\mathrm{MCH}$ in the CA1 field, Sita et al. (2016) investigated the effects of $\mathrm{MCH}$ immunoneutralization centered on the CA3. Animals with impaired $\mathrm{MCH}$ signaling in the $\mathrm{CA} 3$ were more effective recalling the place where food was buried during the training phase, while control animals took longer and dug in other sites before attempting the right quadrant. The most reasonable explanation for the observed results is that animals could perceive olfactory cues indicating that there was no food in the test trial, so control animals searched for food in the arena while $\mathrm{MCH}$-impaired animals were unable to incorporate these cues to the previously stored information.

What circuit could underlie the observations of Sita et al. (2016)? According to Easton et al. (2012), there is a dichotomy between the encoding of new afferent information into preexisting memories, a phenomenon dependent of the entorhinal cortex and the CA1 field, and the retrieval of memories, based on CA3-CA1 interactions. Therefore, the inhibition of $\mathrm{MCH}$ in the $\mathrm{CA} 3$ may have favored this latter circuit, allowing the animal to better recruit the location of the buried empty petri dish, but impairing its ability to perceive the absence of food, indicating that normal $\mathrm{MCH}$ activity in the CA3 is necessary for regular integration of sensory information to acquired memories, in a similar fashion to cholinergic input (Easton et al., 2012).

\section{OTHER BEHAVIORS}

The actions of $\mathrm{MCH}$ on behaviors not related to energy homeostasis are less understood, although recent developments 
began to shine some light in these other actions (Figure 6). There is ample morphological support for $\mathrm{MCH}$ actions over sexual behavior, as $\mathrm{MCH}$-ir fibers are found in multiple relevant areas such as the AVPV, the MPOA, the AHA, and the ME (Bittencourt et al., 1992), where they may contact GnRH-ir neurons (Williamson-Hughes et al., 2005; Ward et al., 2009; Wu et al., 2009; Skrapits et al., 2015) and fibers (Williamson-Hughes et al., 2005; Ward et al., 2009). This MCH-GnRH interaction may underlie the modulation of $\mathrm{MCH}$ over $\mathrm{LH}$ release, although the exact mechanism through which this modulation occurs is still contentious (Gonzalez et al., 1997; Murray et al., 2000a, 2006; Tsukamura et al., 2000; Wu et al., 2009). In this regard, the MPOA and the IHy seem to play an important role (Murray et al., 2000b,c, 2006).

Not only $\mathrm{MCH}$ appears to impact the sexual physiology, but steroidal hormones seem to influence the $\mathrm{MCH}$ peptidergic system as well. In OVX rats supplemented with EB, the orexigenic effects of $\mathrm{MCH}$ are impaired in comparison to male and OVXonly rats, an observation that can be extended to physiological conditions (Messina et al., 2006; Santollo and Eckel, 2008, 2013). These effects of E2, however, appear to be dependent on polysynaptic mechanisms, since $\mathrm{MCH}$ neurons are close to ER $\alpha$-containing neurons, but they do not co-localize (Muschamp and Hull, 2007; Santollo and Eckel, 2013). Steroidal cues also play a role in $\mathrm{MCH}$ fiber density, as the number of $\mathrm{MCH}$-ir fibers in the external layer of the $\mathrm{ME}(\mathrm{MEe})$ is increased in the diestrus and proestrus, in sharp contrast to what is observed during estrus or in males (Chiocchio et al., 2001; Gallardo et al., 2004).

In conclusion, although there is an important amount of information regarding the relationship between $\mathrm{MCH}$ and sexual behavior, especially through the modulation of gonadotropin release, further experiments will be necessary to understand the exact mechanism of $\mathrm{MCH}$ action in animals in their natural environment, in light of the complex influence that steroidal hormones and sites of action appear to exert. It is noteworthy, as others have pointed (Naufahu et al., 2013), that $\mathrm{MCH}$ actions on sexual behavior probably incur in important survival gains by better coordinating the time for animals to engage in reproductive behaviors, since doing so in states of negative energy balance could incur in danger to the animal's life and their potential offspring.

There is also evidence for $\mathrm{MCH}$ actions on maternal behavior and the lactation period. The very first functional clue was presented by Parkes and Vale (1993), that applied MCH and NEI in a NH cell culture and found that the amount of OXT secreted increases around 188 and 245\%, respectively. Adams et al. (2011) described that female $\mathrm{Pmch}^{-/-}$mice show a higher litter postpartum mortality $\left(31.1 \pm 13.6 \% \mathrm{Pmch}^{-/-}\right.$vs. $7.3 \pm 4.2 \%$ WT), an observation that they attribute to the reduced capability of the female to integrate olfactory stimuli. Alvisi et al. (2016) report that pup-suckling stimulus does not elicit $\mathrm{MCH}$ neurons activation or increase the number of $\mathrm{MCH}$-ir neurons in the MPOA, suggesting that hormonal and other sensorial cues have prevalent interactions modulating the $\mathrm{MCH}$ system during this period. Recently, Benedetto et al. (2014) and Alachkar et al. (2016) demonstrated that $\mathrm{MCH}$ has an important role in the expression of maternal behavior through the MPOA, acting as a promoter of maternal behavior in the early postpartum period and a selective inhibitor of appetitive components of this behavior at late stages. It is noteworthy that Alachkar et al. (2016) describe impaired maternal aggression in $\mathrm{Mchr}^{-/-}$, while Adams et al. (2011) report that $\mathrm{Pmch}^{-/-}$animals have increased levels of aggression when on a group and faster initial aggressive response. Besides those two works, very little is known about a possible role for $\mathrm{MCH}$ in aggression and defensive behavior, so further experimental investigation is necessary to better elucidate this aspect of the $\mathrm{MCH}$ peptidergic system.

Although not motivated behaviors per se, sleep and arousal are essential processes with an important interplay with motivated behaviors, especially through hypothalamic circuits (Saper et al., 2001, 2005). Intraventricular injections of $\mathrm{MCH}$ increase the amount of time spent by rats in REM and slow-wave sleep (Verret et al., 2003). Likewise, the subcutaneous treatment with an MCHR1 antagonist decreases the amount of REM sleep (Ahnaou et al., 2008). Recently, Vetrivelan et al. (2016) demonstrated that $\mathrm{MCH}$ neuronal activity increases REM sleep, but $\mathrm{MCH}$ neurons are necessary for the normal wake-REM sleep rhythm. $\mathrm{MCH}$ actions on sleep depend on projections to the DR (Lagos et al., 2009, 2011), oral pontine reticular nucleus (Torterolo et al., 2009), horizontal limb of the diagonal band of Broca (Lagos et al., 2012), ventrolateral preoptic area (Benedetto et al., 2013) and to the locus coeruleus (Monti et al., 2015). Sleep-promoting actions of $\mathrm{MCH}$ may be important to reduce activity and conserve energy in states of negative energy balance, when the animal has a propensity to increase arousal to maximize foraging. The results of Willie et al. (2008) suggest that this is the case, as fasted $\mathrm{Pmch}^{-/-}$animals have increased activity and exaggerated REM sleep time reduction.

\section{CONCLUDING REMARKS}

In this review, we highlighted the circuitry that underlies the integrative functions of $\mathrm{MCH}$. The $\mathrm{MCH}$ circuitry is an extremely relevant tool because it allows us to dissect some of the multiple inputs that reach the hypothalamus and the many targets that can be affected by this system. However, this exactly plethora of connections makes this peptidergic system overwhelmingly hard to study and to comprehend, as each experimental manipulation affects multiple physiological variables. It is not uncommon, therefore, to observe slightly distinct experimental approaches resulting in widely different results, sometimes to the point of an apparent contradiction between obtained data. Although diverse reasons can be pointed to explain this, it is remarkable to us that studying the $\mathrm{MCH}$ neuron as a single entity may be hampering the field. Studies like those of Burdakov et al. (2005) and Hausen et al. (2016) tell us that subsets of MCH neurons differ in respect to their electrophysiological responses to varied stimuli, while studies like those of Elias et al. (2008) and Kampe et al. (2009) provide evidence for differential projection fields among $\mathrm{MCH}$ neurons. This may be in the heart of our inability to turn $\mathrm{MCH}$ into a valid pharmacological target to treat obesity or psychiatric disorders (Méndez-Andino and Wos, 2007; Högberg et al., 2012), as the desirable effects of MCHR1 


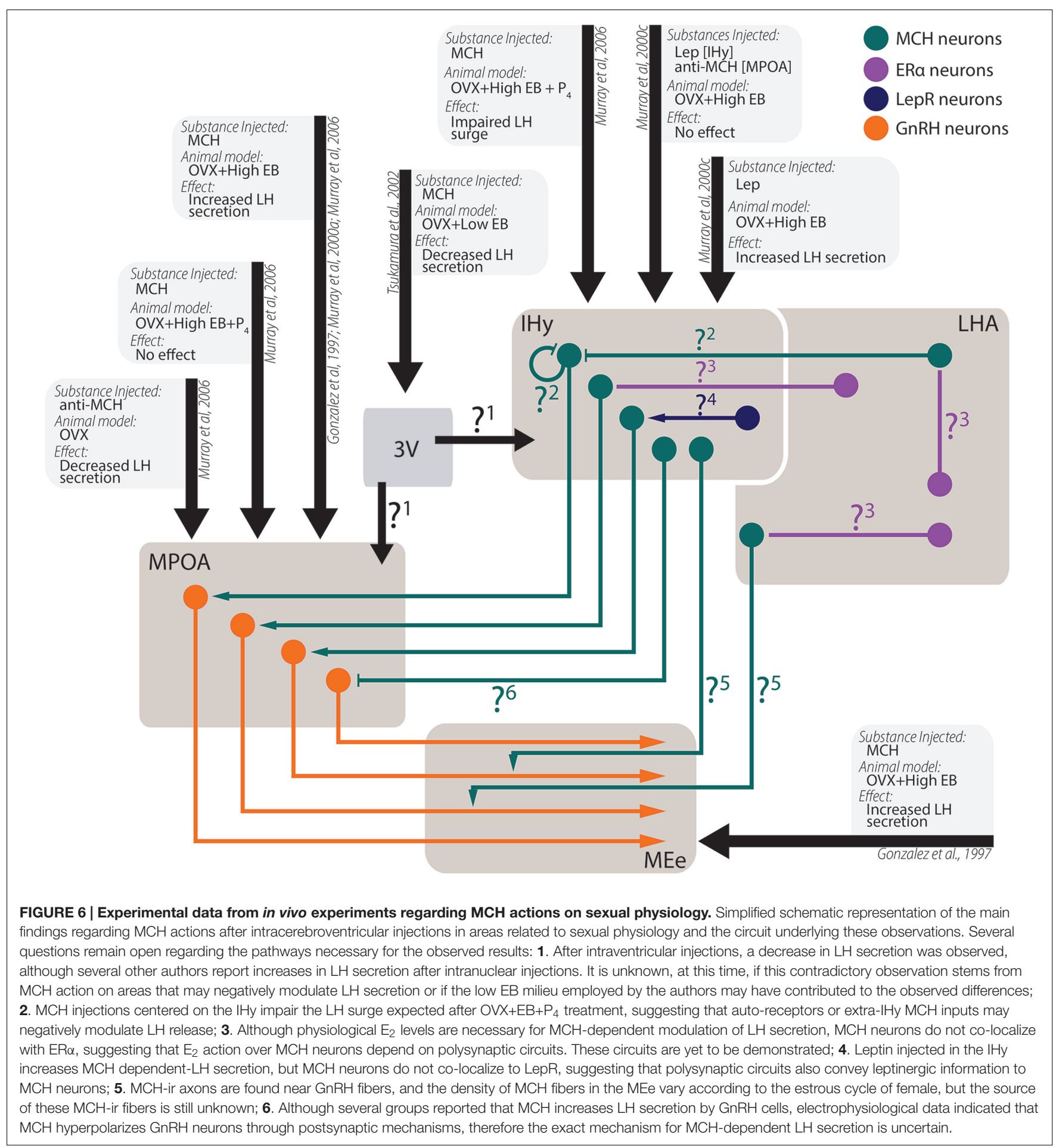

antagonism are overshadowed by unwanted side effects. Not everything is grim in the future of $\mathrm{MCH}$ study, however, as works that better characterize the morphology, the hodology and the chemical signatures of the LHA, such as those of Swanson et al. (2005), Hahn (2010), and Hahn and Swanson (2015), are important steps in the better characterization of the intrinsic properties of subsets of $\mathrm{MCH}$ neurons. Once we understand their variability, we may be able to design drugs to act only on the desired aspects of the $\mathrm{MCH}$ peptidergic system and preserve those that must not be manipulated. Accurate morphological data, nevertheless, continues to be an essential tool for the better understanding of peptidergic systems and of the integrative properties of the hypothalamus as a whole. 


\section{AUTHOR CONTRIBUTIONS}

GD and JB contributed with the writing of this article and approved it for publication.

\section{FUNDING}

This article was supported by Fundação de Amparo à Pesquisa do Estado de São Paulo (São Paulo Research Foundation - FAPESP) grants $\mathrm{n}^{\circ}$ 2016/02748-0 (GD) and $\mathrm{n}^{\circ}$ 2010/52068-0 (JB). We would also like to thank Coordenação de Aperfeiçoamento de

\section{REFERENCES}

Abbott, C. R., Kennedy, A. R., Wren, A. M., Rossi, M., Murphy, K. G., Seal, L. J., et al. (2003). Identification of hypothalamic nuclei involved in the orexigenic effect of melanin-concentrating hormone. Endocrinology 144, 3943-3949. doi: 10.1210/en.2003-0149

Adamantidis, A., Thomas, E., Foidart, A., Tyhon, A., Coumans, B., Minet, A., et al. (2005). Disrupting the melanin-concentrating hormone receptor 1 in mice leads to cognitive deficits and alterations of NMDA receptor function. Eur. J. Neurosci. 21, 2837-2844. doi: 10.1111/j.1460-9568.2005.04100.x

Adams, A. C., Domouzoglou, E. M., Chee, M. J., Segal-Lieberman, G., Pissios, P., and Maratos-Flier, E. (2011). Ablation of the hypothalamic neuropeptide melanin concentrating hormone is associated with behavioral abnormalities that reflect impaired olfactory integration. Behav. Brain Res. 224, 195-200. doi: 10.1016/j.bbr.2011.05.039

Ahnaou, A., Drinkenburg, W. H., Bouwknecht, J. A., Alcazar, J., Steckler, T., and Dautzenberg, F. M. (2008). Blocking melanin-concentrating hormone MCH 1 receptor affects rat sleep-wake architecture. Eur. J. Pharmacol. 579, 177-188. doi: 10.1016/j.ejphar.2007.10.017

Alachkar, A., Alhassen, L., Wang, Z., Wang, L., Onouye, K., Sanathara, N., et al. (2016). Inactivation of the melanin concentrating hormone system impairs maternal behavior. Eur. Neuropsychopharmacol. 26, 1826-1835. doi: 10.1016/ j.euroneuro.2016.08.014

Alberto, C. O., Trask, R. B., and Hirasawa, M. (2011). Dopamine acts as a partial agonist for $\alpha 2 \mathrm{~A}$ adrenoceptor in melanin-concentrating hormone neurons. J. Neurosci. 31, 10671-10676. doi: 10.1523/JNEUROSCI.6245-10.2011

Alexandre, C., Andermann, M. L., and Scammell, T. E. (2013). Control of arousal by the orexin neurons. Curr. Opin. Neurobiol. 23, 752-759. doi: 10.1016/j.conb. 2013.04.008

Allen, Y. S., Adrian, T. E., Allen, J. M., Tatemoto, K., Crow, T. J., Bloom, S. R., et al. (1983). Neuropeptide Y distribution in the rat brain. Science 221, 877-879. doi: $10.1126 /$ science.6136091

Alon, T., and Friedman, J. M. (2006). Late-onset leanness in mice with targeted ablation of melanin concentrating hormone neurons. J. Neurosci. 26, 389-397. doi: 10.1523/JNEUROSCI.1203-05.2006

Alvisi, R., Diniz, G., Da-Silva, J., Bittencourt, J., and Felicio, L. (2016). Sucklinginduced Fos activation and melanin-concentrating hormone immunoreactivity during late lactation. Life Sci. 148, 241-246. doi: 10.1016/j.lfs.2016.02.038

Amano, M., Takahashi, A., Oka, Y., Yamanome, T., Kawauchi, H., and Yamamori, K. (2003). Immunocytochemical localization and ontogenic development of melanin-concentrating hormone in the brain of a pleuronectiform fish, the barfin flounder. Cell Tissue Res. 311, 71-77. doi: 10.1007/s00441-002-0660-6

Amores, A., Catchen, J., Ferrara, A., Fontenot, Q., and Postlethwait, J. H. (2011) Genome evolution and meiotic maps by massively parallel DNA sequencing: spotted gar, an outgroup for the teleost genome duplication. Genetics 188, 799-808. doi: 10.1534/genetics.111.127324

Andén, N. E., Dahlström, A., Fuxe, K., Larsson, K., Olson, L., and Ungerstedt, U. (1966). Ascending monoamine neurons to the telencephalon and diencephalon. Acta Physiol. 67, 313-326. doi: 10.1111/j.1748-1716.1966.tb03318.x

Andersen, A. C., Pelletier, G., Eberle, A. N., Leroux, P., Jegou, S., and Vaudry, H. (1986). Localization of melanin-concentrating hormone-like immunoreactivity
Pessoal de Nível Superior (Agency for the Advancement of Higher Education - CAPES, Grant \#848/15). JB is an Investigator with the Conselho Nacional de Desenvolvimento Científico e Tecnológico (National Council for Scientific and Technological Development - CNPq).

\section{ACKNOWLEDGMENT}

We would like to thank Dr. Luciane Sita for her contributions revising some sections of this article, especially those regarding reproductive behavior.

in the brain and pituitary of the frog Rana ridibunda. Peptides 7, 941-951. doi: 10.1016/0196-9781(86)90119-1

Apergis-Schoute, J., Iordanidou, P., Faure, C., Jego, S., Schöne, C., Aitta-Aho, T., et al. (2015). Optogenetic evidence for inhibitory signaling from orexin to MCH neurons via local microcircuits. J. Neurosci. 35, 5435-5441. doi: 10.1523/ JNEUROSCI.5269-14.2015

Banks, W. A., Kastin, A. J., Huang, W., Jaspan, J. B., and Maness, L. M. (1996). Leptin enters the brain by a saturable system independent of insulin. Peptides 17, 305-311. doi: 10.1016/0196-9781(96)00025-3

Barson, J. R., Morganstern, I., and Leibowitz, S. F. (2012). Neurobiology of consummatory behavior: mechanisms underlying overeating and drug use. ILAR J. 53, 35-58. doi: 10.1093/ilar.53.1.35

Barth, S. W., Riediger, T., Lutz, T. A., and Rechkemmer, G. (2003). Differential effects of amylin and salmon calcitonin on neuropeptide gene expression in the lateral hypothalamic area and the arcuate nucleus of the rat. Neurosci. Lett. 341, 131-134. doi: 10.1016/S0304-3940(03)00190-3

Batten, T., and Baker, B. (1988). Melanin-concentrating hormone (MCH) immunoreactive hypophysial neurosecretory system in the teleost Poecilia latipinna: light and electron microscopic study. Gen. Comp. Endocrinol. 70, 193-205. doi: 10.1016/0016-6480(88)90140-2

Benedetto, L., Pereira, M., Ferreira, A., and Torterolo, P. (2014). Melaninconcentrating hormone in the medial preoptic area reduces active components of maternal behavior in rats. Peptides 58, 20-25. doi: 10.1016/j.peptides.2014. 05.012

Benedetto, L., Rodriguez-Servetti, Z., Lagos, P., D’almeida, V., Monti, J. M., and Torterolo, P. (2013). Microinjection of melanin concentrating hormone into the lateral preoptic area promotes non-REM sleep in the rat. Peptides 39, 11-15. doi: 10.1016/j.peptides.2012.10.005

Berman, J. R., Skariah, G., Maro, G. S., Mignot, E., and Mourrain, P. (2009) Characterization of two melanin-concentrating hormone genes in zebrafish reveals evolutionary and physiological links with the mammalian $\mathrm{MCH}$ system. J. Comp. Neurol. 517, 695-710. doi: 10.1002/cne.22171

Berthoud, H. R. (2011). Metabolic and hedonic drives in the neural control of appetite: who is the boss? Curr. Opin. Neurobiol. 21, 888-896. doi: 10.1016/j. conb.2011.09.004

Bird, D. J., and Baker, B. I. (1989). An immunological study of the secretory activity of neurons producing melanin-concentrating hormone in a teleost. Neuroscience 28, 245-251. doi: 10.1016/0306-4522(89)90248-0

Bird, D. J., Potter, I. C., Sower, S. A., and Baker, B. I. (2001). The distribution of melanin-concentrating hormone in the lamprey brain. Gen. Comp. Endocrinol. 121, 232-241. doi: 10.1006/gcen.2001.7609

Bittencourt, J., and Celis, M. E. (2008). Anatomy, function and regulation of neuropeptide EI (NEI). Peptides 29, 1441-1450. doi: 10.1016/j.peptides.2008. 03.012

Bittencourt, J. C. (2011). Anatomical organization of the melanin-concentrating hormone peptide family in the mammalian brain. Gen. Comp. Endocrinol. 172, 185-197. doi: 10.1016/j.ygcen.2011.03.028

Bittencourt, J. C., and Elias, C. F. (1998). Melanin-concentrating hormone and neuropeptide EI projections from the lateral hypothalamic area and zona incerta to the medial septal nucleus and spinal cord: a study using multiple neuronal tracers. Brain Res. 805, 1-19. doi: 10.1016/S0006-8993(98) 00598-8 
Bittencourt, J. C., Frigo, L., Rissman, R. A., Casatti, C. A., Nahon, J. L., and Bauer, J. A. (1998). The distribution of melanin-concentrating hormone in the monkey brain (Cebus apella). Brain Res. 804, 140-143. doi: 10.1016/S0006-8993(98) 00662-3

Bittencourt, J. C., Presse, F., Arias, C., Peto, C., Vaughan, J., Nahon, J. L., et al. (1992). The melanin-concentrating hormone system of the rat brain: an immuno- and hybridization histochemical characterization. J. Comp. Neurol. 319, 218-245. doi: 10.1002/cne.903190204

Bittencourt, J. C., Vaughan, J., Arias, C., Rissman, R. A., Vale, W. W., and Sawchenko, P. E. (1999). Urocortin expression in rat brain: evidence against a pervasive relationship of Urocortin-Containing projections with targets bearing type 2 CRF receptors. J. Comp. Neurol. 415, 285-312. doi: 10.1002/(SICI)10969861(19991220)415:3<285::AID-CNE1>3.0.CO;2-0

Blessing, W. W., and Chalmers, J. P. (1979). Direct projection of catecholamine (presumably dopamine)-containing neurons from hypothalamus to spinal cord. Neurosci. Lett. 11, 35-40. doi: 10.1016/0304-3940(79)90052-1

Bradley, R. L., Kokkotou, E. G., Maratos-Flier, E., and Cheatham, B. (2000). Melanin-concentrating hormone regulates leptin synthesis and secretion in rat adipocytes. Diabetes Metab. Res. Rev. 49, 1073-1077. doi: 10.2337/diabetes.49.7. 1073

Brailoiu, G. C., Dun, S. L., Brailoiu, E., Inan, S., Yang, J., Chang, J. K., et al. (2007). Nesfatin-1: distribution and interaction with a $\mathrm{G}$ protein-coupled receptor in the rat brain. Endocrinology 148, 5088-5094. doi: 10.1210/en.2007-0701

Broberger, C., De Lecea, L., Sutcliffe, J. G., and Hokfelt, T. (1998). Hypocretin/orexin- and melanin-concentrating hormone-expressing cells form distinct populations in the rodent lateral hypothalamus: relationship to the neuropeptide $\mathrm{Y}$ and agouti gene-related protein systems. J. Comp. Neurol. 402, 460-474. doi: 10.1002/(SICI)1096-9861(19981228)402:4<460:: AID-CNE3>3.0.CO;2-S

Brownstein, M. J., Palkovits, M., Saavedra, J. M., Bassiri, R. M., and Utiger, R. D. (1974). Thyrotropin-releasing hormone in specific nuclei of rat brain. Science 185, 267-269. doi: 10.1126/science.185.4147.267

Brüning, J. C., Gautam, D., Burks, D. J., Gillette, J., Schubert, M., Orban, P. C., et al. (2000). Role of brain insulin receptor in control of body weight and reproduction. Science 289, 2122-2125. doi: 10.1126/science.289.5487. 2122

Burdakov, D., Gerasimenko, O., and Verkhratsky, A. (2005). Physiological changes in glucose differentially modulate the excitability of hypothalamic melaninconcentrating hormone and orexin neurons in situ. J. Neurosci. 25, 2429-2433. doi: 10.1523/JNEUROSCI.4925-04.2005

Burdakov, D., Karnani, M. M., and Gonzalez, A. (2013). Lateral hypothalamus as a sensor-regulator in respiratory and metabolic control. Physiol. Behav. 121, 117-124. doi: 10.1016/j.physbeh.2013.03.023

Cabanac, M., and Johnson, K. (1983). Analysis of a conflict between palatability and cold exposure in rats. Physiol. Behav. 31, 249-253. doi: 10.1016/0031-9384(83) 90128-2

Campfield, L. A., Smith, F. J., Guisez, Y., Devos, R., and Burn, P. (1995). Recombinant mouse OB protein: evidence for a peripheral signal linking adiposity and central neural networks. Science 269, 546. doi: 10.1126/science. 7624778

Cardot, J., Fellmann, D., and Bugnon, C. (1994). Melanin-concentrating hormoneproducing neurons in reptiles. Gen. Comp. Endocrinol. 94, 23-32. doi: 10.1006/ gcen.1994.1056

Cardot, J., Griffond, B., Blahser, S., and Fellmann, D. (1998). Melaninconcentrating hormone in the cock. Ann. N. Y. Acad. Sci. 839, 631-633. doi: 10.1111/j.1749-6632.1998.tb10900.x

Cardot, J., Griffond, B., Risold, P. Y., Blahser, S., and Fellmann, D. (1999). Melaninconcentrating hormone-producing neurons in birds. J. Comp. Neurol. 411, 239-256. doi: 10.1002/(SICI)1096-9861(19990823)411:2<239::AID-CNE5>3.0. $\mathrm{CO} ; 2-7$

Carlezon, W. A., and Thomas, M. J. (2009). Biological substrates of reward and aversion: a nucleus accumbens activity hypothesis. Neuropharmacology 56, 122-132. doi: 10.1016/j.neuropharm.2008.06.075

Carnier, J., De Piano, A., De Lima Sanches, P., Tock, L., Do Nascimento, C. M., Oyama, L. M., et al. (2010). The role of orexigenic and anorexigenic factors in an interdisciplinary weight loss therapy for obese adolescents with symptoms of eating disorders. Int. J. Clin. Pract. 64, 784-790. doi: 10.1111/j.1742-1241.2009. 02306.x
Challis, B., Coll, A., Yeo, G., Pinnock, S., Dickson, S. L., Thresher, R., et al. (2004). Mice lacking pro-opiomelanocortin are sensitive to high-fat feeding but respond normally to the acute anorectic effects of peptide-YY336. Proc. Natl. Acad. Sci. U.S.A. 101, 4695-4700. doi: 10.1073/pnas.03069 31101

Chalmers, D. T., Lovenberg, T. W., and De Souza, E. B. (1995). Localization of novel corticotropin-releasing factor receptor (CRF2) mRNA expression to specific subcortical nuclei in rat brain: comparison with CRF1 receptor mRNA expression. J. Neurosci. 15, 6340-6350.

Chee, M. J., Pissios, P., and Maratos-Flier, E. (2013). Neurochemical characterization of neurons expressing melanin-concentrating hormone receptor 1 in the mouse hypothalamus. J. Comp. Neurol. 521, 2208-2234. doi: $10.1002 /$ cne. 23273

Cheung, C. C., Clifton, D. K., and Steiner, R. A. (1997). Proopiomelanocortin neurons are direct targets for leptin in the hypothalamus. Endocrinology 138, 4489-4492. doi: 10.1210/endo.138.10.5570

Chiocchio, S. R., Gallardo, M. G., Louzan, P., Gutnisky, V., and Tramezzani, J. H. (2001). Melanin-concentrating hormone stimulates the release of luteinizing hormone-releasing hormone and gonadotropins in the female rat acting at both median eminence and pituitary levels. Biol. Reprod. 64, 1466-1472. doi: 10.1095/biolreprod64.5.1466

Chometton, S., Franchi, G., Houdayer, C., Mariot, A., Poncet, F., Fellmann, D., et al. (2014). Different distributions of preproMCH and hypocretin/orexin in the forebrain of the pig (Sus scrofa domesticus). J. Chem. Neuroanat. 6, 72-82. doi: 10.1016/j.jchemneu.2014.08.001

Chung, S., Hopf, F. W., Nagasaki, H., Li, C. Y., Belluzzi, J. D., Bonci, A., et al. (2009). The melanin-concentrating hormone system modulates cocaine reward. Proc. Natl. Acad. Sci. U.S.A. 106, 6772-6777. doi: 10.1073/pnas.08113 31106

Chung, S., Wong, T., Nagasaki, H., and Civelli, O. (2010). Acute homeostatic responses to increased fat consumption in MCH1R knockout mice. J. Mol. Neurosci. 42, 459-463. doi: 10.1007/s12031-010-9358-5

Cippitelli, A., Karlsson, C., Shaw, J. L., Thorsell, A., Gehlert, D. R., and Heilig, M. (2010). Suppression of alcohol self-administration and reinstatement of alcohol seeking by melanin-concentrating hormone receptor 1 (MCH1-R) antagonism in Wistar rats. Psychopharmacology 211, 367-375. doi: 10.1007/s00213-0101891-y

Clark, J. T., Kalra, P. S., Crowley, W. R., and Kalra, S. P. (1984). Neuropeptide Y and human pancreatic polypeptide stimulate feeding behavior in rats. Endocrinology 115, 427-429. doi: 10.1210/endo-115-1-427

Clegg, D. J., Air, E. L., Benoit, S. C., Sakai, R. S., Seeley, R. J., and Woods, S. C. (2003). Intraventricular melanin-concentrating hormone stimulates water intake independent of food intake. Am. J. Physiol. Regul. Integr. Comp. Physiol. 284, R494-R499. doi: 10.1152/ajpregu.00399.2002

Comoli, E., Ribeiro-Barbosa, E. R., and Canteras, N. S. (2003). Predatory hunting and exposure to a live predator induce opposite patterns of Fos immunoreactivity in the PAG. Behav. Brain Res. 138, 17-28. doi: 10.1016/ S0166-4328(02)00197-3

Conductier, G., Nahon, J.-L., and Guyon, A. (2011). Dopamine depresses melanin concentrating hormone neuronal activity through multiple effects on $\alpha 2$ noradrenergic, D1 and D2-like dopaminergic receptors. Neuroscience 178, 89-100. doi: 10.1016/j.neuroscience.2011.01.030

Cvetkovic, V., Brischoux, F., Griffond, B., Bernard, G., Jacquemard, C., Fellmann, D., et al. (2003). Evidence of melanin-concentrating hormonecontaining neurons supplying both cortical and neuroendocrine projections. Neuroscience 116, 31-35. doi: 10.1016/S0306-4522(02)00557-2

Dahlström, A., and Fuxe, K. (1963). Evidence for the existence of monoaminecontaining neurons in the central nervous system. I. Demonstration of monoamines in the cell bodies of brain stem neurons. Acta Physiol. Scand. Suppl. 232, 231-255.

Dallvechia-Adams, S., Kuhar, M. J., and Smith, Y. (2002). Cocaine- and amphetamine-regulated transcript peptide projections in the ventral midbrain: colocalization with gamma-aminobutyric acid, melanin-concentrating hormone, dynorphin, and synaptic interactions with dopamine neurons. J. Comp. Neurol. 448, 360-372. doi: 10.1002/cne.10268

De Vry, J., and Schreiber, R. (2000). Effects of selected serotonin 5-HT 1 and 5-HT 2 receptor agonists on feeding behavior: possible mechanisms of action. Neurosci. Biobehav. Rev. 24, 341-353. doi: 10.1016/S0149-7634(99)00083-4 
Debons, A. F., Silver, L., Cronkite, E. P., Johnson, H. A., Brecher, G., Tenzer, D., et al. (1962). Localization of gold in mouse brain in relation to gold thioglucose obesity. Am. J. Physiol. 202, 743-750.

Della-Zuana, O., Presse, F., Ortola, C., Duhault, J., Nahon, J. L., and Levens, N. (2002). Acute and chronic administration of melanin-concentrating hormone enhances food intake and body weight in Wistar and Sprague-Dawley rats. Int. J. Obes. Relat. Metab. Disord. 26, 1289-1295. doi: 10.1038/sj.ijo. 0802079

Dobolyi, A. (2009). Central amylin expression and its induction in rat dams. J. Neurochem. 111, 1490-1500. doi: 10.1111/j.1471-4159.2009.06422.x

Domingos, A. I., Sordillo, A., Dietrich, M. O., Liu, Z. W., Tellez, L. A., Vaynshteyn, J., et al. (2013). Hypothalamic melanin concentrating hormone neurons communicate the nutrient value of sugar. Elife 2:e01462. doi: 10.7554/ eLife.01462

Drazen, D. L., Coolen, L. M., Strader, A. D., Wortman, M. D., Woods, S. C., and Seeley, R. J. (2004). Differential effects of adrenalectomy on melaninconcentrating hormone and orexin A. Endocrinology 145, 3404-3412. doi: 10. 1210/en.2003-1760

Duncan, E. A., Proulx, K., and Woods, S. C. (2005). Central administration of melanin-concentrating hormone increases alcohol and sucrose/quinine intake in rats. Alcohol. Clin. Exp. Res. 29, 958-964. doi: 10.1097/01.ALC.0000167741. 42353.10

Duncan, E. A., Rider, T. R., Jandacek, R. J., Clegg, D. J., Benoit, S. C., Tso, P., et al. (2006). The regulation of alcohol intake by melanin-concentrating hormone in rats. Pharmacol. Biochem. Behav. 85, 728-735. doi: 10.1016/j.pbb.2006. 11.004

Duncan, E. A., Sorrell, J. E., Adamantidis, A., Rider, T., Jandacek, R. J., Seeley, R. J., et al. (2007). Alcohol drinking in $\mathrm{MCH}$ receptor-1-deficient mice. Alcoholism 31, 1325-1337. doi: 10.1111/j.1530-0277.2007.00427.x

Easton, A., Douchamps, V., Eacott, M., and Lever, C. (2012). A specific role for septohippocampal acetylcholine in memory? Neuropsychologia 50, 3156-3168. doi: 10.1016/j.neuropsychologia.2012.07.022

Elias, C. F., and Bittencourt, J. C. (1997). Study of the origins of melaninconcentrating hormone and neuropeptide EI immunoreactive projections to the periaqueductal gray matter. Brain Res. 755, 255-271. doi: 10.1016/S00068993(97)00104-2

Elias, C. F., Lee, C. E., Kelly, J. F., Ahima, R. S., Kuhar, M., Saper, C. B., et al. (2001). Characterization of CART neurons in the rat and human hypothalamus. J. Comp. Neurol. 432, 1-19. doi: 10.1002/cne.1085

Elias, C. F., Saper, C. B., Maratos-Flier, E., Tritos, N. A., Lee, C., Kelly, J., et al. (1998). Chemically defined projections linking the mediobasal hypothalamus and the lateral hypothalamic area. J. Comp. Neurol. 402, 442-459. doi: 10.1002/ (SICI) 1096-9861(19981228)402:4<442::AID-CNE2>3.0.CO;2-R

Elias, C. F., Sita, L. V., Zambon, B. K., Oliveira, E. R., Vasconcelos, L. A., and Bittencourt, J. C. (2008). Melanin-concentrating hormone projections to areas involved in somatomotor responses. J. Chem. Neuroanat. 35, 188-201. doi: 10.1016/j.jchemneu.2007.10.002

Fekete, C., Mihaly, E., Luo, L. G., Kelly, J., Clausen, J. T., Mao, Q., et al. (2000). Association of cocaine- and amphetamine-regulated transcriptimmunoreactive elements with thyrotropin-releasing hormone-synthesizing neurons in the hypothalamic paraventricular nucleus and its role in the regulation of the hypothalamic-pituitary-thyroid axis during fasting. J. Neurosci. 20, 9224-9234.

Ferrario, C. R., Labouèbe, G., Liu, S., Nieh, E. H., Routh, V. H., Xu, S., et al. (2016). Homeostasis meets motivation in the battle to control food intake. J. Neurosci. 36, 11469-11481. doi: 10.1523/JNEUROSCI.2338-16.2016

Foo, H., and Mason, P. (2005). Sensory suppression during feeding. Proc. Natl. Acad. Sci. U.S.A. 102, 16865-16869. doi: 10.1073/pnas.0506226102

Fort, P., Salvert, D., Hanriot, L., Jego, S., Shimizu, H., Hashimoto, K., et al. (2008). The satiety molecule nesfatin- 1 is co-expressed with melanin concentrating hormone in tuberal hypothalamic neurons of the rat. Neuroscience 155, 174-181. doi: 10.1016/j.neuroscience.2008.05.035

Francis, K., and Baker, B. I. (1995). Developmental changes in melaninconcentrating hormone in Rana temporaria. Gen. Comp. Endocrinol. 98, 157-165. doi: 10.1006/gcen.1995.1056

Freedman, M. R., Horwitz, B. A., and Stern, J. S. (1986). Effect of adrenalectomy and glucocorticoid replacement on development of obesity. Am. J. Physiol. 250, R595-R607.
Frenk, H., and Rogers, G. H. (1979). The suppressant effects of naloxone on food and water intake in the rat. Behav. Neural Biol. 26, 23-40. doi: 10.1016/S01631047(79)92855-3

Fu, L.-Y., Acuna-Goycolea, C., and Van Den Pol, A. N. (2004). Neuropeptide Y inhibits hypocretin/orexin neurons by multiple presynaptic and postsynaptic mechanisms: tonic depression of the hypothalamic arousal system. J. Neurosci. 24, 8741-8751. doi: 10.1523/JNEUROSCI.2268-04.2004

Furudono, Y., Ando, C., Yamamoto, C., Kobashi, M., and Yamamoto, T. (2006). Involvement of specific orexigenic neuropeptides in sweetener-induced overconsumption in rats. Behav. Brain Res. 175, 241-248. doi: 10.1016/j.bbr. 2006.08.031

Gallardo, M. G., Chiocchio, S. R., and Tramezzani, J. H. (2004). Changes of melanin-concentrating hormone related to LHRH release in the median eminence of rats. Brain Res. 1030, 152-158. doi: 10.1016/j.brainres.2004.10.005

Gao, X. B., and van den Pol, A. N. (2001). Melanin concentrating hormone depresses synaptic activity of glutamate and GABA neurons from rat lateral hypothalamus. J. Physiol. 533, 237-252. doi: 10.1111/j.1469-7793.2001. 0237b.x

Gavrila, A., Chan, J. L., Miller, L. C., Heist, K., Yiannakouris, N., and Mantzoros, C. S. (2005). Circulating melanin-concentrating hormone, agouti-related protein, and alpha-melanocyte-stimulating hormone levels in relation to body composition: alterations in response to food deprivation and recombinant human leptin administration. J. Clin. Endocrinol. Metab. 90, 1047-1054. doi: 10.1210/jc.2004-1124

Georgescu, D., Sears, R. M., Hommel, J. D., Barrot, M., Bolanos, C. A., Marsh, D. J., et al. (2005). The hypothalamic neuropeptide melanin-concentrating hormone acts in the nucleus accumbens to modulate feeding behavior and forced-swim performance. J. Neurosci. 25, 2933-2940. doi: 10.1523/JNEUROSCI.1714-04. 2005

Glick, M., Segal-Lieberman, G., Cohen, R., and Kronfeld-Schor, N. (2009). Chronic $\mathrm{MCH}$ infusion causes a decrease in energy expenditure and body temperature, and an increase in serum IGF-1 levels in mice. Endocrine 36, 479-485. doi: 10.1007/s12020-009-9252-5

Goforth, P. B., Leinninger, G. M., Patterson, C. M., Satin, L. S., and Myers, M. G. (2014). Leptin acts via lateral hypothalamic area neurotensin neurons to inhibit orexin neurons by multiple GABA-independent mechanisms. J. Neurosci. 34 11405-11415. doi: 10.1523/JNEUROSCI.5167-13.2014

Gomori, A., Ishihara, A., Ito, M., Mashiko, S., Matsushita, H., Yumoto, M., et al. (2003). Chronic intracerebroventricular infusion of $\mathrm{MCH}$ causes obesity in mice. Melanin-concentrating hormone. Am. J. Physiol. Endocrinol. Metab. 284, E583-E588. doi: 10.1152/ajpendo.00350.2002

Gonzalez, M. I., Baker, B. I., and Wilson, C. A. (1997). Stimulatory effect of melanin-concentrating hormone on luteinising hormone release. Neuroendocrinology 66, 254-262. doi: 10.1159/000127246

Green, P. K., Wilkinson, C. W., and Woods, S. C. (1992). Intraventricular corticosterone increases the rate of body weight gain in underweight adrenalectomized rats. Endocrinology 130, 269-275. doi: 10.1210/endo.130.1. 1727703

Gröneveld, D., Balm, P., and Wendelaar Bonga, S. (1995). Identification, cellular localization and in vitro release of a novel teleost melanin-concentrating hormone gene-related peptide. Neuroendocrinology 62, 498-505. doi: 10.1159/ 000127040

Guesdon, B., Paradis, E., Samson, P., and Richard, D. (2009). Effects of intracerebroventricular and intra-accumbens melanin-concentrating hormone agonism on food intake and energy expenditure. Am. J. Physiol. Regul. Integr. Comp. Physiol. 296, R469-R475. doi: 10.1152/ajpregu.90556.2008

Haemmerle, C. A., Campos, A. M., and Bittencourt, J. C. (2015). Melaninconcentrating hormone inputs to the nucleus accumbens originate from distinct hypothalamic sources and are apposed to GABAergic and cholinergic cells in the Long-Evans rat brain. Neuroscience 289, 392-405. doi: 10.1016/j. neuroscience.2015.01.014

Hahn, J. D. (2010). Comparison of melanin-concentrating hormone and hypocretin/orexin peptide expression patterns in a current parceling scheme of the lateral hypothalamic zone. Neurosci. Lett. 468, 12-17. doi: 10.1016/j.neulet. 2009.10.047

Hahn, J. D., and Swanson, L. W. (2015). Connections of the juxtaventromedial region of the lateral hypothalamic area in the male rat. Front. Syst. Neurosci. 9:66. doi: 10.3389/fnsys.2015.00066 
Halaas, J. L., Gajiwala, K. S., Maffei, M., and Cohen, S. L. (1995). Weight-reducing effects of the plasma protein encoded by the obese gene. Science 269, 543 . doi: $10.1126 /$ science. 7624777

Hancock, M. B. (1976). Cells of origin of hypothalamo-spinal projections in the rat. Neurosci. Lett. 3, 179-184. doi: 10.1016/0304-3940(76)90070-7

Hausen, A. C., Ruud, J., Jiang, H., Hess, S., Varbanov, H., Kloppenburg, P., et al. (2016). Insulin-dependent activation of $\mathrm{MCH}$ neurons impairs locomotor activity and insulin sensitivity in obesity. Cell Rep. 17, 2512-2521. doi: 10.1016/ j.celrep.2016.11.030

Hervieu, G., and Nahon, J. L. (1995). Pro-melanin concentrating hormone messenger ribonucleic acid and peptides expression in peripheral tissues of the rat. Neuroendocrinology 61, 348-364. doi: 10.1159/000126857

Hervieu, G. J., Cluderay, J. E., Harrison, D., Meakin, J., Maycox, P., Nasir, S., et al. (2000). The distribution of the mRNA and protein products of the melaninconcentrating hormone $(\mathrm{MCH})$ receptor gene, slc-1, in the central nervous system of the rat. Eur. J. Neurosci. 12, 1194-1216. doi: 10.1046/j.1460-9568. 2000.00008.x

Hill, J., Duckworth, M., Murdock, P., Rennie, G., Sabido-David, C., Ames, R. S., et al. (2001). Molecular cloning and functional characterization of $\mathrm{MCH} 2$, a novel human MCH receptor. J. Biol. Chem. 276, 20125-20129. doi: 10.1074/jbc. M102068200

Hogben, L., and Slome, D. (1935). The Pigmentary Effector System. VIII-The Dual Receptive Mechanism of the Amphibian Background Response. London: The Royal Society.

Högberg, T., Frimurer, T. M., and Sasmal, P. K. (2012). Melanin concentrating hormone receptor 1 (MCHR1) antagonists-Still a viable approach for obesity treatment? Bioorg. Med. Chem. Lett. 22, 6039-6047. doi: 10.1016/j.bmcl.2012. 08.025

Holtzman, S. G. (1974). Behavioral effects of separate and combined administration of naloxone and d-amphetamine. J. Pharmacol. Exp. Ther. 189, 51-60.

Horjales-Araujo, E., Hellysaz, A., and Broberger, C. (2014). Lateral hypothalamic thyrotropin-releasing hormone neurons: distribution and relationship to histochemically defined cell populations in the rat. Neuroscience 277, 87-102. doi: 10.1016/j.neuroscience.2014.06.043

Hosoya, Y., and Matsushita, M. (1979). Identification and distribution of the spinal and hypophyseal projection neurons in the paraventricular nucleus of the rat. A light and electron microscopic study with the horseradish peroxidase method. Exp. Brain Res. 35, 315-331. doi: 10.1007/BF00236618

Huang, H., Acuna-Goycolea, C., Li, Y., Cheng, H., Obrietan, K., and Van Den Pol, A. N. (2007). Cannabinoids excite hypothalamic melanin-concentrating hormone but inhibit hypocretin/orexin neurons: implications for cannabinoid actions on food intake and cognitive arousal. J. Neurosci. 27, 4870-4881. doi: 10.1523/JNEUROSCI.0732-07.2007

Huang, H., and van den Pol, A. N. (2007). Rapid direct excitation and longlasting enhancement of NMDA response by group I metabotropic glutamate receptor activation of hypothalamic melanin-concentrating hormone neurons. J. Neurosci. 27, 11560-11572. doi: 10.1523/JNEUROSCI.2147-07.2007

Hubschle, T., Mckinley, M. J., and Oldfield, B. J. (1998). Efferent connections of the lamina terminalis, the preoptic area and the insular cortex to submandibular and sublingual gland of the rat traced with pseudorabies virus. Brain Res. 806, 219-231. doi: 10.1016/S0006-8993(98)00765-3

Huesa, G., Van Den Pol, A. N., and Finger, T. E. (2005). Differential distribution of hypocretin (orexin) and melanin-concentrating hormone in the goldfish brain. J. Comp. Neurol. 488, 476-491. doi: 10.1002/cne.20610

Jacobowitz, D. M., and O'Donohue, T. L. (1978). alpha-Melanocyte stimulating hormone: immunohistochemical identification and mapping in neurons of rat brain. Proc. Natl. Acad. Sci. U.S.A. 75, 6300-6304. doi: 10.1073/pnas.75.12.6300

Jaworski, J. N., Kozel, M. A., Philpot, K. B., and Kuhar, M. J. (2003). Intra-accumbal injection of CART (cocaine-amphetamine regulated transcript) peptide reduces cocaine-induced locomotor activity. J. Pharmacol. Exp. Ther. 307, 1038-1044. doi: $10.1124 /$ jpet.103.052332

Johnson, S. W., and North, R. A. (1992). Two types of neurone in the rat ventral tegmental area and their synaptic inputs. J. Physiol. 450, 455. doi: 10.1113/ jphysiol.1992.sp019136

Júnior, E. D. D. S., Da Silva, A. V., Da Silva, K. R., Haemmerle, C. A., Batagello, D. S., Da Silva, J. M., et al. (2015). The centrally projecting edingerwestphal nucleus-I: efferents in the rat brain. J. Chem. Neuroanat. 68, 22-38. doi: 10.1016/j.jchemneu.2015.07.002
Kampe, J., Tschop, M. H., Hollis, J. H., and Oldfield, B. J. (2009). An anatomic basis for the communication of hypothalamic, cortical and mesolimbic circuitry in the regulation of energy balance. Eur. J. Neurosci. 30, 415-430. doi: 10.1111/j. 1460-9568.2009.06818.x

Karlsson, C., Aziz, A. M. A., Rehman, F., Pitcairn, C., Barchiesi, R., Barbier, E., et al. (2016). Melanin-concentrating hormone and Its MCH-1 receptor: relationship between effects on alcohol and caloric intake. Alcoholism 40, 2199-2207. doi: 10.1111/acer.13181

Karlsson, C., Zook, M., Ciccocioppo, R., Gehlert, D. R., Thorsell, A., Heilig, M., et al. (2012). Melanin-concentrating hormone receptor 1 (MCH1-R) antagonism: reduced appetite for calories and suppression of addictive-like behaviors. Pharmacol. Biochem. Behav. 102, 400-406. doi: 10.1016/j.pbb.2012. 06.010

Kawauchi, H., and Baker, B. I. (2004). Melanin-concentrating hormone signaling systems in fish. Peptides 25, 1577-1584. doi: 10.1016/j.peptides.2004.03.025

Kawauchi, H., Kawazoe, I., Tsubokawa, M., Kishida, M., and Baker, B. I. (1983). Characterization of melanin-concentrating hormone in chum salmon pituitaries. Nature 305, 321-323. doi: 10.1038/305321a0

Kelley, A. E., Baldo, B. A., Pratt, W. E., and Will, M. J. (2005). Corticostriatalhypothalamic circuitry and food motivation: integration of energy, action and reward. Physiol. Behav. 86, 773-795. doi: 10.1016/j.physbeh.2005. 08.066

Kennedy, A. R., Todd, J. F., Stanley, S. A., Abbott, C. R., Small, C. J., Ghatei, M. A., et al. (2001). Melanin-concentrating hormone (MCH) suppresses thyroid stimulating hormone (TSH) release, in vivo and in vitro, via the hypothalamus and the pituitary. Endocrinology 142, 3265-3268. doi: 10.1210/endo.142. 7.8374

Khorooshi, R. M., and Klingenspor, M. (2005). Neuronal distribution of melaninconcentrating hormone, cocaine- and amphetamine-regulated transcript and orexin B in the brain of the Djungarian hamster (Phodopus sungorus). J. Chem. Neuroanat. 29, 137-148. doi: 10.1016/j.jchemneu.2004.10.003

Kim, J.-H., Creekmore, E., and Vezina, P. (2003). Microinjection of CART peptide 55-102 into the nucleus accumbens blocks amphetamine-induced locomotion. Neuropeptides 37, 369-373. doi: 10.1016/j.npep.2003.10.001

Knollema, S., Brown, E. R., Vale, W., and Sawchenko, P. E. (1992). Novel hypothalamic and preoptic sites of prepro-melanin-concentrating hormone messenger ribonucleic Acid and Peptide expression in lactating rats. J. Neuroendocrinol. 4, 709-717. doi: 10.1111/j.1365-2826.1992.tb00222.x

Kohler, C., Haglund, L., and Swanson, L. W. (1984). A diffuse alpha MSHimmunoreactive projection to the hippocampus and spinal cord from individual neurons in the lateral hypothalamic area and zona incerta. J. Comp. Neurol. 223, 501-514. doi: 10.1002/cne.902230404

Kong, D., Vong, L., Parton, L. E., Ye, C., Tong, Q., Hu, X., et al. (2010). Glucose stimulation of hypothalamic MCH neurons involves K ATP channels, is modulated by UCP2, and regulates peripheral glucose homeostasis. Cell Metab. 12, 545-552. doi: 10.1016/j.cmet.2010.09.013

Korotkova, T. M., Sergeeva, O. A., Eriksson, K. S., Haas, H. L., and Brown, R. E. (2003). Excitation of ventral tegmental area dopaminergic and nondopaminergic neurons by orexins/hypocretins. J. Neurosci. 23, 7-11.

Koylu, E. O., Couceyro, P. R., Lambert, P. D., and Kuhar, M. J. (1998). Cocaine- and amphetamine-regulated transcript peptide immunohistochemical localization in the rat brain. J. Comp. Neurol. 391, 115-132. doi: 10.1002/(SICI)10969861(19980202)391:1<115::AID-CNE10>3.0.CO;2-X

Koylu, E. O., Couceyro, P. R., Lambert, P. D., Ling, N. C., Desouza, E. B., and Kuhar, M. J. (1997). Immunohistochemical localization of novel CART peptides in rat hypothalamus, pituitary and adrenal gland. J. Neuroendocrinol. 9, 823-833. doi: 10.1046/j.1365-2826.1997.00651.x

Kozicz, T., Bittencourt, J. C., May, P. J., Reiner, A., Gamlin, P. D., Palkovits, M., et al. (2011). The Edinger-Westphal nucleus: a historical, structural, and functional perspective on a dichotomous terminology. J. Comp. Neurol. 519, 1413-1434. doi: $10.1002 /$ cne. 22580

Kozicz, T., Yanaihara, H., and Arimura, A. (1998). Distribution of urocortin-like immunoreactivity in the central nervous system of the rat. J. Comp. Neurol. 391, 1-10. doi: 10.1002/(SICI)1096-9861(19980202)391:1<1::AID-CNE1>3.0. $\mathrm{CO} ; 2-6$

Krause, M., German, P. W., Taha, S. A., and Fields, H. L. (2010). A pause in nucleus accumbens neuron firing is required to initiate and maintain feeding. J. Neurosci. 30, 4746-4756. doi: 10.1523/JNEUROSCI.0197-10.2010 
Kristensen, P., Judge, M. E., Thim, L., Ribel, U., Christjansen, K. N., Wulff, B. S., et al. (1998). Hypothalamic CART is a new anorectic peptide regulated by leptin. Nature 393, 72-76. doi: 10.1038/29993

Lagos, P., Monti, J. M., Jantos, H., and Torterolo, P. (2012). Microinjection of the melanin-concentrating hormone into the lateral basal forebrain increases REM sleep and reduces wakefulness in the rat. Life Sci. 90, 895-899. doi: 10.1016/j.lfs. 2012.04.019

Lagos, P., Torterolo, P., Jantos, H., Chase, M. H., and Monti, J. M. (2009). Effects on sleep of melanin-concentrating hormone $(\mathrm{MCH})$ microinjections into the dorsal raphe nucleus. Brain Res. 1265, 103-110. doi: 10.1016/j.brainres.2009. 02.010

Lagos, P., Torterolo, P., Jantos, H., and Monti, J. M. (2011). Immunoneutralization of melanin-concentrating hormone $(\mathrm{MCH})$ in the dorsal raphe nucleus: effects on sleep and wakefulness. Brain Res. 1369, 112-118. doi: 10.1016/j.brainres. 2010.11.027

Lázár, G., Maderdrut, J. L., and Merchenthaler, I. (2002). Distribution of melaninconcentrating hormone-like immunoreactivity in the central nervous system of Rana esculenta. Brain Res. Bull. 57, 401-407. doi: 10.1016/S0361-9230(01) 00675-X

Lechan, R. M., and Fekete, C. (2006). The TRH neuron: a hypothalamic integrator of energy metabolism. Prog. Brain Res. 153, 209-235. doi: 10.1016/S00796123(06)53012-2

Leffert, J. D., Newgard, C. B., Okamoto, H., Milburn, J. L., and Luskey, K. L. (1989). Rat amylin: cloning and tissue-specific expression in pancreatic islets. Proc. Natl. Acad. Sci. U.S.A. 86, 3127-3130. doi: 10.1073/pnas.86. 9.3127

Legendre, A., Papakonstantinou, E., Roy, M.-C., Richard, D., and Harris, R. B. (2007). Differences in response to corticotropin-releasing factor after short-and long-term consumption of a high-fat diet. Am. J. Physiol. Regul. Integr. Comp. Physiol. 293, R1076-R1085. doi: 10.1152/ajpregu.00592.2006

Legradi, G., Emerson, C. H., Ahima, R. S., Rand, W. M., Flier, J. S., and Lechan, R. M. (1998). Arcuate nucleus ablation prevents fasting-induced suppression of ProTRH mRNA in the hypothalamic paraventricular nucleus. Neuroendocrinology 68, 89-97. doi: 10.1159/000054354

Legradi, G., and Lechan, R. M. (1998). The arcuate nucleus is the major source for neuropeptide Y-innervation of thyrotropin-releasing hormone neurons in the hypothalamic paraventricular nucleus. Endocrinology 139, 3262-3270. doi: 10.1210/endo.139.7.6113

Legradi, G., and Lechan, R. M. (1999). Agouti-related protein containing nerve terminals innervate thyrotropin-releasing hormone neurons in the hypothalamic paraventricular nucleus. Endocrinology 140, 3643-3652. doi: 10.1210/endo.140.8.6935

Leibowitz, S. F., and Alexander, J. T. (1998). Hypothalamic serotonin in control of eating behavior, meal size, and body weight. Biol. Psychiatry 44, 851-864. doi: 10.1016/S0006-3223(98)00186-3

Leibowitz, S. F., and Brown, L. L. (1980). Histochemical and pharmacological analysis of catecholaminergic projections to the perifornical hypothalamus in relation to feeding inhibition. Brain Res. 201, 315-345. doi: 10.1016/00068993(80)91038-0

Leinninger, G. M., Jo, Y.-H., Leshan, R. L., Louis, G. W., Yang, H., Barrera, J. G., et al. (2009). Leptin acts via leptin receptor-expressing lateral hypothalamic neurons to modulate the mesolimbic dopamine system and suppress feeding. Cell Metab. 10, 89-98. doi: 10.1016/j.cmet.2009.06.011

Leinninger, G. M., Opland, D. M., Jo, Y.-H., Faouzi, M., Christensen, L., Cappellucci, L. A., et al. (2011). Leptin action via neurotensin neurons controls orexin, the mesolimbic dopamine system and energy balance. Cell Metab. 14, 313-323. doi: 10.1016/j.cmet.2011.06.016

Leloup, C., Arluison, M., Lepetit, N., Cartier, N., Marfaing-Jallat, P., Ferré, P., et al. (1994). Glucose transporter 2 (GLUT 2): expression in specific brain nuclei. Brain Res. 638, 221-226. doi: 10.1016/0006-8993(94)90653-X

Lewis, K., Li, C., Perrin, M., Blount, A., Kunitake, K., Donaldson, C., et al. (2001). Identification of urocortin III, an additional member of the corticotropinreleasing factor (CRF) family with high affinity for the CRF2 receptor. Proc. Natl. Acad. Sci. U.S.A. 98, 7570-7575. doi: 10.1073/pnas.121165198

Li, Y., and van den Pol, A. N. (2006). Differential target-dependent actions of coexpressed inhibitory dynorphin and excitatory hypocretin/orexin neuropeptides. J. Neurosci. 26, 13037-13047. doi: 10.1523/JNEUROSCI.338006.2006
Lima, F. F., Sita, L. V., Oliveira, A. R., Costa, H. C., Da Silva, J. M., Mortara, R. A., et al. (2013). Hypothalamic melanin-concentrating hormone projections to the septo-hippocampal complex in the rat. J. Chem. Neuroanat. 47, 1-14. doi: 10.1016/j.jchemneu.2012.10.003

Liu, H., Kishi, T., Roseberry, A. G., Cai, X., Lee, C. E., Montez, J. M., et al. (2003). Transgenic mice expressing green fluorescent protein under the control of the melanocortin-4 receptor promoter. J. Neurosci. 23, 7143-7154.

Louis, G. W., Leinninger, G. M., Rhodes, C. J., and Myers, M. G. Jr. (2010). Direct innervation and modulation of orexin neurons by lateral hypothalamic LepRb neurons. J. Neurosci. 30, 11278-11287. doi: 10.1523/JNEUROSCI.1340-10.2010

Ludwig, D. S., Mountjoy, K. G., Tatro, J. B., Gillette, J. A., Frederich, R. C., Flier, J. S., et al. (1998). Melanin-concentrating hormone: a functional melanocortin antagonist in the hypothalamus. Am. J. Physiol. 274, E627-E633.

Ludwig, D. S., Tritos, N. A., Mastaitis, J. W., Kulkarni, R., Kokkotou, E., Elmquist, J., et al. (2001). Melanin-concentrating hormone overexpression in transgenic mice leads to obesity and insulin resistance. J. Clin. Invest. 107, 379-386. doi: 10.1172/JCI10660

Lutz, T., Mollet, A., Rushing, P., Riediger, T., and Scharrer, E. (2001). The anorectic effect of a chronic peripheral infusion of amylin is abolished in area postrema/nucleus of the solitary tract (AP/NTS) lesioned rats. Int. J. Obes. 25, 1005. doi: $10.1038 /$ sj.ijo. 0801664

Mahler, S. V., Moorman, D. E., Smith, R. J., James, M. H., and Aston-Jones, G. (2014). Motivational activation: a unifying hypothesis of orexin/hypocretin function. Nat. Neurosci. 17, 1298-1303. doi: 10.1038/nn.3810

Mancera, J. M., and Fernandez-Llebrez, P. (1995). Development of melaninconcentrating hormone-immunoreactive elements in the brain of gilthead seabream (Sparus aurata). Cell Tissue Res. 282, 523-526. doi: 10.1007/ BF00318885

Maolood, N., and Meister, B. (2010). Nociceptin/orphanin FQ peptide in hypothalamic neurones associated with the control of feeding behaviour. J. Neuroendocrinol. 22, 75-82. doi: 10.1111/j.1365-2826.2009.01946.x

Marsh, D. J., Weingarth, D. T., Novi, D. E., Chen, H. Y., Trumbauer, M. E., Chen, A. S., et al. (2002). Melanin-concentrating hormone 1 receptor-deficient mice are lean, hyperactive, and hyperphagic and have altered metabolism. Proc. Natl. Acad. Sci. U.S.A. 99, 3240-3245. doi: 10.1073/pnas.052706899

Matsuda, K., Kojima, K., Shimakura, S.-I., Miura, T., Uchiyama, M., Shioda, S., et al. (2009). Relationship between melanin-concentrating hormone-and neuropeptide Y-containing neurons in the goldfish hypothalamus. Comp. Biochem. Physiol. A Mol. Integr. Physiol. 153, 3-7. doi: 10.1016/j.cbpa.2008. 10.002

Mayer, D., Price, D., Lewis, V., and Gebhart, G. (1978). Insulin receptors are widely distributed in the central nervous system of the rat. Nature 272, 27.

Méndez-Andino, J. L., and Wos, J. A. (2007). MCH-R1 antagonists: what is keeping most research programs away from the clinic? Drug Discov. Today 12, 972-979. doi: 10.1016/j.drudis.2007.08.010

Mercer, J. G., Hoggard, N., Williams, L. M., Lawrence, C. B., Hannah, L. T., Morgan, P. J., et al. (1996). Coexpression of leptin receptor and preproneuropeptide Y mRNA in arcuate nucleus of mouse hypothalamus. J. Neuroendocrinol. 8, 733-735. doi: 10.1046/j.1365-2826.1996.05161.x

Messina, M. M., Boersma, G., Overton, J. M., and Eckel, L. A. (2006). Estradiol decreases the orexigenic effect of melanin-concentrating hormone in ovariectomized rats. Physiol. Behav. 88, 523-528. doi: 10.1016/j.physbeh.2006. 05.002

Mietlicki-Baase, E. G., Olivos, D. R., Jeffrey, B. A., and Hayes, M. R. (2015). Cooperative interaction between leptin and amylin signaling in the ventral tegmental area for the control of food intake. Am. J. Physiol. Endocrinol. Metab. 308, E1116-E1122. doi: 10.1152/ajpendo.00087.2015

Mietlicki-Baase, E. G., Rupprecht, L. E., Olivos, D. R., Zimmer, D. J., Alter, M. D., Pierce, R. C., et al. (2013). Amylin receptor signaling in the ventral tegmental area is physiologically relevant for the control of food intake. Neuropsychopharmacology 38, 1685-1697. doi: 10.1038/npp.2013.66

Miller, C. L., Hruby, V. J., Matsunaga, T. O., and Bickford, P. C. (1993). Alpha$\mathrm{MSH}$ and $\mathrm{MCH}$ are functional antagonists in a CNS auditory gating paradigm. Peptides 14, 431-440. doi: 10.1016/0196-9781(93)90128-4

Minth, C. D., Qiu, H., Akil, H., Watson, S. J., and Dixon, J. E. (1989). Two precursors of melanin-concentrating hormone: DNA sequence analysis and in situ immunochemical localization. Proc. Natl. Acad. Sci. U.S.A. 86, 4292-4296. doi: $10.1073 /$ pnas.86.11.4292 
Mizuno, N. (1991). Electron microscopic identification of axons containing melanin-concentrating hormone in the lamprey, Lampetra fluviatilis L. Neurosci. Lett. 128, 249-252. doi: 10.1016/0304-3940(91)90272-U

Mizusawa, K., Amiya, N., Yamaguchi, Y., Takabe, S., Amano, M., Breves, J. P., et al. (2012). Identification of mRNAs coding for mammalian-type melaninconcentrating hormone and its receptors in the scalloped hammerhead shark Sphyrna lewini. Gen. Comp. Endocrinol. 179, 78-87. doi: 10.1016/j.ygcen.2012. 07.023

Modirrousta, M., Mainville, L., and Jones, B. E. (2005). Orexin and MCH neurons express c-Fos differently after sleep deprivation vs. recovery and bear different adrenergic receptors. Eur. J. Neurosci. 21, 2807-2816. doi: 10.1111/j.1460-9568. 2005.04104.x

Moffett, M. C., Song, J., and Kuhar, M. J. (2011). CART peptide inhibits locomotor activity induced by simultaneous stimulation of D1 and D2 receptors, but not by stimulation of individual dopamine receptors. Synapse 65, 1-7. doi: 10.1002/ syn. 20815

Momiyama, T., Todo, N., and Sasa, M. (1993). A mechanism underlying dopamine D1 and D2 receptor-mediated inhibition of dopaminergic neurones in the ventral tegmental area in vitro. Br. J. Pharmacol. 109, 933-940. doi: 10.1111/ j.1476-5381.1993.tb13710.x

Monti, J. M., Lagos, P., Jantos, H., and Torterolo, P. (2015). Increased REM sleep after intra-locus coeruleus nucleus microinjection of melanin-concentrating hormone (MCH) in the rat. Prog. Neuropsychopharmacol. Biol. Psychiatry 56, 185-188. doi: 10.1016/j.pnpbp.2014.09.003

Monzon, M. E., De Souza, M. M., Izquierdo, L. A., Izquierdo, I., Barros, D. M., and De Barioglio, S. R. (1999). Melanin-concentrating hormone (MCH) modifies memory retention in rats. Peptides 20, 1517-1519. doi: 10.1016/S0196-9781(99) 00164-3

Moore, R., and Bloom, F. (1979). Central catecholamine neuron systems: anatomy and physiology of the norepinephrine and epinephrine systems. Annu. Rev. Neurosci. 2, 113-168. doi: 10.1146/annurev.ne.02.030179.000553

Morganstern, I., Chang, G.-Q., Chen, Y.-W., Barson, J., Zhiyu, Y., Hoebel, B., et al. (2010a). Role of melanin-concentrating hormone in the control of ethanol consumption: region-specific effects revealed by expression and injection studies. Physiol. Behav. 101, 428-437. doi: 10.1016/j.physbeh.2010.07.009

Morganstern, I., Chang, G.-Q., Karatayev, O., and Leibowitz, S. F. (2010b). Increased orexin and melanin-concentrating hormone expression in the perifornical lateral hypothalamus of rats prone to overconsuming a fat-rich diet. Pharmacol. Biochem. Behav. 96, 413-422. doi: 10.1016/j.pbb.2010.06.013

Mul, J. D., La Fleur, S. E., Toonen, P. W., Afrasiab-Middelman, A., Binnekade, R., Schetters, D., et al. (2011). Chronic loss of melanin-concentrating hormone affects motivational aspects of feeding in the rat. PLoS ONE 6:e19600. doi: 10.1371/journal.pone. 0019600

Muroya, S., Yada, T., Shioda, S., and Takigawa, M. (1999). Glucose-sensitive neurons in the rat arcuate nucleus contain neuropeptide Y. Neurosci. Lett. 264, 113-116. doi: 10.1016/S0304-3940(99)00185-8

Murray, J. F., Adan, R. A., Walker, R., Baker, B. I., Thody, A. J., Nijenhuis, W. A., et al. (2000a). Melanin-concentrating hormone, melanocortin receptors and regulation of luteinizing hormone release. J. Neuroendocrinol. 12, 217-223. doi: $10.1046 /$ j.1365-2826.2000.00440.x

Murray, J. F., Baker, B. I., Levy, A., and Wilson, C. A. (2000b). The influence of gonadal steroids on pre-pro melanin-concentrating hormone mRNA in female rats. J. Neuroendocrinol. 12, 53-59.

Murray, J. F., Mercer, J. G., Adan, R. A., Datta, J. J., Aldairy, C., Moar, K. M., et al. (2000c). The effect of leptin on luteinizing hormone release is exerted in the zona incerta and mediated by melanin-concentrating hormone. J. Neuroendocrinol. 12, 1133-1139.

Murray, J. F., Hahn, J., Kennedy, A., Small, C., Bloom, S., Haskell-Luevano, C., et al. (2006). Evidence for a stimulatory action of melanin-concentrating hormone on luteinising hormone release involving $\mathrm{MCH} 1$ and melanocortin-5 receptors. J. Neuroendocrinol. 18, 157-167. doi: 10.1111/j.1365-2826.2005.01397.x

Muschamp, J. W., and Hull, E. M. (2007). Melanin concentrating hormone and estrogen receptor- $\alpha$ are coexstensive but not coexpressed in cells of male rat hypothalamus. Neurosci. Lett. 427, 123-126. doi: 10.1016/j.neulet.2007.09.031

Myers, M. G. Jr., and Olson, D. P. (2012). Central nervous system control of metabolism. Nature 491, 357-363. doi: 10.1038/nature11705

Nahon, J. L., Presse, F., Bittencourt, J. C., Sawchenko, P. E., and Vale, W. (1989). The rat melanin-concentrating hormone messenger ribonucleic acid encodes multiple putative neuropeptides coexpressed in the dorsolateral hypothalamus. Endocrinology 125, 2056-2065. doi: 10.1210/endo-125-4-2056

Naito, N., Nakai, Y., Kawauchi, H., and Hayashi, Y. (1985). Immunocytochemical identification of melanin-concentrating hormone in the brain and pituitary gland of the teleost fishes Oncorhynchus keta and Salmo gairdneri. Cell Tissue Res. 242, 41-48. doi: 10.1007/BF00225561

Nakamura, S., Tsumori, T., Yokota, S., Oka, T., and Yasui, Y. (2009). Amygdaloid axons innervate melanin-concentrating hormone-and orexincontaining neurons in the mouse lateral hypothalamus. Brain Res. 1278, 66-74. doi: 10.1016/j.brainres.2009.04.049

Naufahu, J., Alzaid, F., Brito, M. F., Doslikova, B., Valencia, T. M., Cunliffe, A., et al. (2017). Melanin concentrating hormone in peripheral circulation in the human. J. Endocrinol. 232, 513-523. doi: 10.1530/JOE-16-0240

Naufahu, J., Cunliffe, A. D., and Murray, J. F. (2013). The roles of melaninconcentrating hormone in energy balance and reproductive function: are they connected? Reproduction 146, R141-R150. doi: 10.1530/REP-12-0385

Niswender, K. D., Morrison, C. D., Clegg, D. J., Olson, R., Baskin, D. G., Myers, M. G., et al. (2003). Insulin activation of phosphatidylinositol 3-kinase in the hypothalamic arcuate nucleus: a key mediator of insulin-induced anorexia. Diabetes Metab. Res. Rev. 52, 227-231. doi: 10.2337/diabetes.52.2.227

O'Connor, E. C., Kremer, Y., Lefort, S., Harada, M., Pascoli, V., Rohner, C., et al. (2015). Accumbal D1R neurons projecting to lateral hypothalamus authorize feeding. Neuron 88, 553-564. doi: 10.1016/j.neuron.2015.09.038

Oldfield, B. J., Giles, M. E., Watson, A., Anderson, C., Colvill, L. M., and Mckinley, M. J. (2002). The neurochemical characterisation of hypothalamic pathways projecting polysynaptically to brown adipose tissue in the rat. Neuroscience 110 , 515-526. doi: 10.1016/S0306-4522(01)00555-3

Ollmann, M. M., Wilson, B. D., Yang, Y. K., Kerns, J. A., Chen, Y., Gantz, I., et al. (1997). Antagonism of central melanocortin receptors in vitro and in vivo by agouti-related protein. Science 278, 135-138. doi: 10.1126/science.278.5335.135

Ono, M., Wada, C., Oikawa, I., Kawazoe, I., and Kawauchi, H. (1988). Structures of two kinds of mRNA encoding the chum salmon melanin-concentrating hormone. Gene 71, 433-438. doi: 10.1016/0378-1119(88)90060-1

Ono, T., Nishino, H., Sasaka, K., Muramoto, K., Yano, I., and Simpson, A. (1978). Paraventricular nucleus connections to spinal cord and pituitary. Neurosci. Lett. 10, 141-146. doi: 10.1016/0304-3940(78)90025-3

Oswald, K. D., Murdaugh, D. L., King, V. L., and Boggiano, M. M. (2011). Motivation for palatable food despite consequences in an animal model of binge eating. Int. J. Eat. Disord. 44, 203-211. doi: 10.1002/eat.20808

Pachoud, B., Adamantidis, A., Ravassard, P., Luppi, P. H., Grisar, T., Lakaye, B., et al. (2010). Major impairments of glutamatergic transmission and long-term synaptic plasticity in the hippocampus of mice lacking the melanin-concentrating hormone receptor-1. J. Neurophysiol. 104, 1417-1425. doi: $10.1152 /$ jn.01052.2009

Palkovits, M., Brownstein, M., Saavedra, J. M., and Axelrod, J. (1974). Norepinephrine and dopamine content of hypothalamic nuclei of the rat. Brain Res. 77, 137-149. doi: 10.1016/0006-8993(74)90810-5

Parkes, D. G., and Vale, W. W. (1993). Contrasting actions of melaninconcentrating hormone and neuropeptide-E-I on posterior pituitary function. Ann. N. Y. Acad. Sci. 680, 588-590. doi: 10.1111/j.1749-6632.1993.tb19746.x

Parks, G. S., Wang, L., Wang, Z., and Civelli, O. (2014). Identification of neuropeptide receptors expressed by melanin-concentrating hormone neurons. J. Comp. Neurol. 522, 3817-3833. doi: 10.1002/cne.23642

Parsons, M. P., and Hirasawa, M. (2011). GIRK channel-mediated inhibition of melanin-concentrating hormone neurons by nociceptin/orphanin FQ. J. Neurophysiol. 105, 1179-1184. doi: 10.1152/jn.00791.2010

Parton, L. E., Ye, C. P., Coppari, R., Enriori, P. J., Choi, B., Zhang, C.-Y., et al. (2007). Glucose sensing by POMC neurons regulates glucose homeostasis and is impaired in obesity. Nature 449, 228-232. doi: 10.1038/nature06098

Pelleymounter, M. A., Cullen, M. J., Baker, M. B., and Hecht, R. (1995). Effects of the obese gene product on body weight regulation in ob/ob mice. Science 269, 540. doi: $10.1126 /$ science. 7624776

Pereira-da-Silva, M., Torsoni, M. A., Nourani, H. V., Augusto, V. D., Souza, C. T., Gasparetti, A. L., et al. (2003). Hypothalamic melanin-concentrating hormone is induced by cold exposure and participates in the control of energy expenditure in rats. Endocrinology 144, 4831-4840. doi: 10.1210/en.2003-0243

Pérez, C. A., Stanley, S. A., Wysocki, R. W., Havranova, J., AhrensNicklas, R., Onyimba, F., et al. (2011). Molecular annotation of integrative 
feeding neural circuits. Cell Metab. 13, 222-232. doi: 10.1016/j.cmet.2010. 12.013

Peyron, C., Tighe, D. K., Van Den Pol, A. N., De Lecea, L., Heller, H. C., Sutcliffe, J. G., et al. (1998). Neurons containing hypocretin (orexin) project to multiple neuronal systems. J. Neurosci. 18, 9996-10015.

Pissios, P., Frank, L., Kennedy, A. R., Porter, D. R., Marino, F. E., Liu, F. F., et al. (2008). Dysregulation of the mesolimbic dopamine system and reward in MCH-/- mice. Biol. Psychiatry 64, 184-191. doi: 10.1016/j.biopsych.2007.12.011

Powell, K., and Baker, B. (1988). Structural studies of nerve terminals containing melanin-concentrating hormone in the eel, Anguilla anguilla. Cell Tissue Res. 251, 433-439. doi: 10.1007/BF00215852

Qu, D., Ludwig, D. S., Gammeltoft, S., Piper, M., Pelleymounter, M. A., Cullen, M. J., et al. (1996). A role for melanin-concentrating hormone in the central regulation of feeding behaviour. Nature 380, 243-247. doi: 10.1038/380243a0

Reiner, D. J., Mietlicki-Baase, E. G., Olivos, D. R., Mcgrath, L. E., Zimmer, D. J., Koch-Laskowski, K., et al. (2017). Amylin acts in the lateral dorsal tegmental nucleus to regulate energy balance through GABA signaling. Biol. Psychiatry (in press). doi: 10.1016/j.biopsych.2016.12.028

Reyes, T., Lewis, K., Perrin, M., Kunitake, K., Vaughan, J., Arias, C., et al. (2001). Urocortin II: a member of the corticotropin-releasing factor (CRF) neuropeptide family that is selectively bound by type 2 CRF receptors. Proc. Natl. Acad. Sci. U.S.A. 98, 2843-2848. doi: 10.1073/pnas.051626398

Rondini, T. A., Donato, J. Jr., Rodrigues Bde, C., Bittencourt, J. C., and Elias, C. F. (2010). Chemical identity and connections of medial preoptic area neurons expressing melanin-concentrating hormone during lactation. J. Chem. Neuroanat. 39, 51-62. doi: 10.1016/j.jchemneu.2009.10.005

Rondini, T. A., Rodrigues Bde, C., De Oliveira, A. P., Bittencourt, J. C., and Elias, C. F. (2007). Melanin-concentrating hormone is expressed in the laterodorsal tegmental nucleus only in female rats. Brain Res. Bull. 74, 21-28. doi: 10.1016/j. brainresbull.2007.04.006

Rossi, M., Choi, S. J., O'shea, D., Miyoshi, T., Ghatei, M. A., and Bloom, S. R. (1997). Melanin-concentrating hormone acutely stimulates feeding, but chronic administration has no effect on body weight. Endocrinology 138, 351-355. doi: 10.1210/endo.138.1.4887

Routh, V. H. (2002). Glucose-sensing neurons: are they physiologically relevant? Physiol. Behav. 76, 403-413. doi: 10.1016/s0031-9384(02)00761-8

Rushing, P. A. (2003). Central amylin signaling and the regulation of energy homeostasis. Curr. Pharm. Des. 9, 819-825. doi: 10.2174/1381612033455387

Saito, Y., Cheng, M., Leslie, F. M., and Civelli, O. (2001). Expression of the melaninconcentrating hormone $(\mathrm{MCH})$ receptor mRNA in the rat brain. J. Comp. Neurol. 435, 26-40. doi: 10.1002/cne.1191

Saito, Y., Nothacker, H. P., Wang, Z., Lin, S. H., Leslie, F., and Civelli, O. (1999). Molecular characterization of the melanin-concentrating-hormone receptor. Nature 400, 265-269. doi: 10.1038/22321

Sakamaki, R., Uemoto, M., Inui, A., Asakawa, A., Ueno, N., Ishibashi, C., et al. (2005). Melanin-concentrating hormone enhances sucrose intake. Int. J. Mol. Med. 15, 1033-1039. doi: $10.3892 /$ ijmm.15.6.1033

Sakurai, T. (2014). The role of orexin in motivated behaviours. Nat. Rev. Neurosci. 15, 719-731. doi: 10.1038/nrn3837

Sakurai, T., Amemiya, A., Ishii, M., Matsuzaki, I., Chemelli, R. M., Tanaka, H., et al. (1998). Orexins and orexin receptors: a family of hypothalamic neuropeptides and G protein-coupled receptors that regulate feeding behavior. Cell 92, 573-585. doi: 10.1016/S0092-8674(00)80949-6

Sano, H., and Yokoi, M. (2007). Striatal medium spiny neurons terminate in a distinct region in the lateral hypothalamic area and do not directly innervate orexin/hypocretin- or melanin-concentrating hormone-containing neurons. J. Neurosci. 27, 6948-6955. doi: 10.1523/JNEUROSCI.0514-07.2007

Santollo, J., and Eckel, L. A. (2008). The orexigenic effect of melanin-concentrating hormone $(\mathrm{MCH})$ is influenced by sex and stage of the estrous cycle. Physiol. Behav. 93, 842-850. doi: 10.1016/j.physbeh.2007.11.050

Santollo, J., and Eckel, L. A. (2013). Oestradiol decreases melanin-concentrating hormone $(\mathrm{MCH})$ and $\mathrm{MCH}$ receptor expression in the hypothalamus of female rats. J. Neuroendocrinol. 25, 570-579. doi: 10.1111/jne.12032

Saper, C. B., Akil, H., and Watson, S. J. (1986). Lateral hypothalamic innervation of the cerebral cortex: immunoreactive staining for a peptide resembling but immunochemically distinct from pituitary/arcuate alpha-melanocyte stimulating hormone. Brain Res. Bull. 16, 107-120. doi: 10.1016/0361-9230(86) 90018-3
Saper, C. B., Chou, T. C., and Elmquist, J. K. (2002). The need to feed: homeostatic and hedonic control of eating. Neuron 36, 199-211. doi: 10.1016/S08966273(02)00969-8

Saper, C. B., Chou, T. C., and Scammell, T. E. (2001). The sleep switch: hypothalamic control of sleep and wakefulness. Trends Neurosci. 24, 726-731. doi: 10.1016/S0166-2236(00)02002-6

Saper, C. B., Loewy, A. D., Swanson, L. W., and Cowan, W. M. (1976). Direct hypothalamo-autonomic connections. Brain Res. 117, 305-312. doi: 10.1016/ 0006-8993(76)90738- 1

Saper, C. B., Scammell, T. E., and Lu, J. (2005). Hypothalamic regulation of sleep and circadian rhythms. Nature 437, 1257-1263. doi: 10.1038/nature04284

Schwartz, M. W., Seeley, R. J., Campfield, L. A., Burn, P., and Baskin, D. G. (1996). Identification of targets of leptin action in rat hypothalamus. J. Clin. Invest. 98, 1101-1106. doi: 10.1172/JCI118891

Scoville, W. B., and Milner, B. (1957). Loss of recent memory after bilateral hippocampal lesions. J. Neurol. Neurosurg. Psychiatry 20, 11-21. doi: 10.1136/ jnnp.20.1.11

Segal-Lieberman, G., Bradley, R. L., Kokkotou, E., Carlson, M., Trombly, D. J. Wang, X., et al. (2003). Melanin-concentrating hormone is a critical mediator of the leptin-deficient phenotype. Proc. Natl. Acad. Sci. U.S.A. 100, 10085-10090. doi: $10.1073 /$ pnas. 1633636100

Sexton, P., Paxinos, G., Kenney, M., Wookey, P., and Beaumont, K. (1994). In vitro autoradiographic localization of amylin binding sites in rat brain. Neuroscience 62, 553-567. doi: 10.1016/0306-4522(94)90388-3

Shimada, M., Tritos, N. A., Lowell, B. B., Flier, J. S., and Maratos-Flier, E. (1998). Mice lacking melanin-concentrating hormone are hypophagic and lean. Nature 396, 670-674. doi: 10.1038/25341

Shutter, J. R., Graham, M., Kinsey, A. C., Scully, S., Luthy, R., and Stark, K. L. (1997). Hypothalamic expression of ART, a novel gene related to agouti, is up-regulated in obese and diabetic mutant mice. Genes Dev. 11, 593-602. doi: 10.1101/gad.11.5.593

Simmons, D. M., and Swanson, L. W. (2008). High-resolution paraventricular nucleus serial section model constructed within a traditional rat brain atlas. Neurosci. Lett. 438, 85-89. doi: 10.1016/j.neulet.2008.04.057

Simmons, D. M., and Swanson, L. W. (2009). Comparison of the spatial distribution of seven types of neuroendocrine neurons in the rat paraventricular nucleus: toward a global 3D model. J. Comp. Neurol. 516, 423-441. doi: 10.1002/ cne.22126

Sita, L. V., Diniz, G. B., Canteras, N. S., Xavier, G. F., and Bittencourt, J. C. (2016). Effect of intrahippocampal administration of anti-melaninconcentrating hormone on spatial food-seeking behavior in rats. Peptides 76, 130-138. doi: 10.1016/j.peptides.2015.12.007

Sita, L. V., Elias, C. F., and Bittencourt, J. C. (2003). Dopamine and melaninconcentrating hormone neurons are distinct populations in the rat rostromedial zona incerta. Brain Res. 970, 232-237. doi: 10.1016/S0006-8993(03) 02345-X

Sita, L. V., Elias, C. F., and Bittencourt, J. C. (2007). Connectivity pattern suggests that incerto-hypothalamic area belongs to the medial hypothalamic system. Neuroscience 148, 949-969. doi: 10.1016/j.neuroscience.2007.07.010

Skrapits, K., Kanti, V., Savanyú, Z., Maurnyi, C., Szenci, O., Horváth, A., et al. (2015). Lateral hypothalamic orexin and melanin-concentrating hormone neurons provide direct input to gonadotropin-releasing hormone neurons in the human. Front. Cell. Neurosci. 9:348. doi: 10.3389/fncel.2015.00348

Smith, D. G., Tzavara, E. T., Shaw, J., Luecke, S., Wade, M., Davis, R., et al. (2005). Mesolimbic dopamine super-sensitivity in melanin-concentrating hormone-1 receptor-deficient mice. J. Neurosci. 25, 914-922. doi: 10.1523/JNEUROSCI. 4079-04.2005

Spina, M., Merlo-Pich, E., Chan, R. K., Basso, A. M., Rivier, J., Vale, W., et al. (1996). Appetite-suppressing effects of urocortin, a CRF-related neuropeptide. Science 273, 1561-1564. doi: 10.1126/science.273.5281.1561

Squire, L. R. (1992). Memory and the hippocampus: a synthesis from findings with rats, monkeys, and humans. Psychol. Rev. 99, 195.

Steinbusch, H. W. M. (1981). Distribution of serotonin-immunoreactivity in the central nervous system of the rat-cell bodies and terminals. Neuroscience 6, 557-618. doi: 10.1016/0306-4522(81)90146-9

Stricker-Krongrad, A., Dimitrov, T., and Beck, B. (2001). Central and peripheral dysregulation of melanin-concentrating hormone in obese Zucker rats. Mol. Brain Res. 92, 43-48. doi: 10.1016/S0169-328X(01)00130-9 
Sun, G., Tian, Z., Murata, T., Narita, K., Honda, K., and Higuchi, T. (2004). Central and peripheral immunoreactivity of melanin-concentrating hormone in hypothalamic obese and lactating rats. J. Neuroendocrinol. 16, 79-83. doi: 10.1111/j.1365-2826.2004.01124.x

Sun, L. L., Zhang, Y., Liu, J. F., Wang, J., Zhu, W. L., Zhao, L. Y., et al. (2013). Role of melanin-concentrating hormone in the nucleus accumbens shell in rats behaviourally sensitized to methamphetamine. Int. J. Neuropsychopharmacol. 16, 1767-1780. doi: 10.1017/S1461145713000072

Suzuki, H., and Yamamoto, T. (2013). Orexin-B-like immunoreactivity localizes in both luteinizing-hormone-containing cells and melanin-concentrating hormone-containing fibers in the red-bellied piranha (Pygocentrus nattereri) pituitary. Cell Tissue Res. 351, 175-182. doi: 10.1007/s00441-012-1 516-3

Swanson, L. W. (2005). Anatomy of the soul as reflected in the cerebral hemispheres: neural circuits underlying voluntary control of basic motivated behaviors. J. Comp. Neurol. 493, 122-131. doi: 10.1002/cne.20733

Swanson, L. W., and Kuypers, H. G. (1980). The paraventricular nucleus of the hypothalamus: cytoarchitectonic subdivisions and organization of projections to the pituitary, dorsal vagal complex, and spinal cord as demonstrated by retrograde fluorescence double-labeling methods. J. Comp. Neurol. 194, 555-570. doi: 10.1002/cne.901940306

Swanson, L. W., Sanchez-Watts, G., and Watts, A. G. (2005). Comparison of melanin-concentrating hormone and hypocretin/orexin mRNA expression patterns in a new parceling scheme of the lateral hypothalamic zone. Neurosci. Lett. 387, 80-84. doi: 10.1016/j.neulet.2005.06.066

Swanson, L. W., and Sawchenko, P. E. (1983). Hypothalamic integration: organization of the paraventricular and supraoptic nuclei. Annu. Rev. Neurosci. 6, 269-324. doi: 10.1146/annurev.ne.06.030183.001413

Tataranni, P. A., Larson, D. E., Snitker, S., Young, J. B., Flatt, J., and Ravussin, E. (1996). Effects of glucocorticoids on energy metabolism and food intake in humans. Am. J. Physiol. Endocrinol. Metab. 271, E317-E325.

Thexton, A. J. (1992). Mastication and swallowing: an overview. Br. Dent. J. 173, 197-206. doi: 10.1038/sj.bdj.4808002

Tillet, Y., Batailler, M., and Fellmann, D. (1996). Distribution of melaninconcentrating hormone $(\mathrm{MCH})$-like immunoreactivity in neurons of the diencephalon of sheep. J. Chem. Neuroanat. 12, 135-145. doi: 10.1016/S08910618(96)00195-0

Torterolo, P., Sampogna, S., and Chase, M. H. (2009). MCHergic projections to the nucleus pontis oralis participate in the control of active (REM) sleep. Brain Res. 1268, 76-87. doi: 10.1016/j.brainres.2009.02.055

Torterolo, P., Sampogna, S., Morales, F. R., and Chase, M. H. (2006). MCHcontaining neurons in the hypothalamus of the cat: searching for a role in the control of sleep and wakefulness. Brain Res. 1119, 101-114. doi: 10.1016/ j.brainres.2006.08.100

Tsukamura, H., Thompson, R. C., Tsukahara, S., Ohkura, S., Maekawa, F., Moriyama, R., et al. (2000). Intracerebroventricular administration of melaninconcentrating hormone suppresses pulsatile luteinizing hormone release in the female rat. J. Neuroendocrinol. 12, 529-534. doi: 10.1046/j.1365-2826.2000. 00482.x

Vallarino, M., Andersen, A. C., Delbende, C., Ottonello, I., Eberle, A. N., and Vaudry, H. (1989). Melanin-concentrating hormone (MCH) immunoreactivity in the brain and pituitary of the dogfish Scyliorhinus canicula. Colocalization with alpha-melanocyte-stimulating hormone (alpha-MSH) in hypothalamic neurons. Peptides 10, 375-382. doi: 10.1016/0196-9781(89) 90046-6

van den Pol, A. N., Acuna-Goycolea, C., Clark, K. R., and Ghosh, P. K. (2004). Physiological properties of hypothalamic MCH neurons identified with selective expression of reporter gene after recombinant virus infection. Neuron 42, 635-652. doi: 10.1016/S0896-6273(04)00251-X

van Houten, M., Posner, B. I., Kopriwa, B. M., and Brawer, J. R. (1980). Insulin binding sites localized to nerve terminals in rat median eminence and arcuate nucleus. Science 207, 1081-1083. doi: 10.1126/science.6986652

Varas, M., Perez, M., Monzon, M. E., and De Barioglio, S. R. (2002a). Melaninconcentrating hormone, hippocampal nitric oxide levels and memory retention. Peptides 23, 2213-2221.

Varas, M., Perez, M., Ramirez, O., and De Barioglio, S. R. (2002b). Melanin concentrating hormone increase hippocampal synaptic transmission in the rat. Peptides 23, 151-155.
Varas, M. M., Perez, M. F., Ramirez, O. A., and De Barioglio, S. R. (2003). Increased susceptibility to LTP generation and changes in NMDA-NR1 and -NR2B subunits mRNA expression in rat hippocampus after $\mathrm{MCH}$ administration. Peptides 24, 1403-1411. doi: 10.1016/j.peptides.2003.09.006

Vaughan, J., Donaldson, C., Bittencourt, J., Perrin, M. H., Lewis, K., Sutton, S., et al. (1995). Urocortin, a mammalian neuropeptide related to fish urotensin I and to corticotropin-releasing factor. Nature 378, 287-292. doi: 10.1038/378287a0

Vaughan, J. M., Fischer, W. H., Hoeger, C., Rivier, J., and Vale, W. (1989). Characterization of melanin-concentrating hormone from rat hypothalamus. Endocrinology 125, 1660-1665. doi: 10.1210/endo-125-3-1660

Verret, L., Goutagny, R., Fort, P., Cagnon, L., Salvert, D., Leger, L., et al. (2003). A role of melanin-concentrating hormone producing neurons in the central regulation of paradoxical sleep. BMC Neurosci. 4:19. doi: 10.1186/14712202-4-19

Verty, A. N., Allen, A. M., and Oldfield, B. J. (2010). The endogenous actions of hypothalamic peptides on brown adipose tissue thermogenesis in the rat. Endocrinology 151, 4236-4246. doi: 10.1210/en.2009-1235

Vetrivelan, R., Kong, D., Ferrari, L. L., Arrigoni, E., Madara, J. C., Bandaru, S. S., et al. (2016). Melanin-concentrating hormone neurons specifically promote rapid eye movement sleep in mice. Neuroscience 336, 102-113. doi: 10.1016/j. neuroscience.2016.08.046

Viale, A., Zhixing, Y., Breton, C., Pedeutour, F., Coquerel, A., Jordan, D., et al. (1997). The melanin-concentrating hormone gene in human: flanking region analysis, fine chromosome mapping, and tissue-specific expression. Mol. Brain Res. 46, 243-255. doi: 10.1016/S0169-328X(97)00018-1

Vidal, L., Blanchard, J., and Morin, L. P. (2005). Hypothalamic and zona incerta neurons expressing hypocretin, but not melanin concentrating hormone, project to the hamster intergeniculate leaflet. Neuroscience 134, 1081-1090. doi: 10.1016/j.neuroscience.2005.03.062

Ward, D. R., Dear, F. M., Ward, I. A., Anderson, S. I., Spergel, D. J., Smith, P. A., et al. (2009). Innervation of gonadotropin-releasing hormone neurons by peptidergic neurons conveying circadian or energy balance information in the mouse. PLOS ONE 4:e5322. doi: 10.1371/journal.pone.00 05322

Waters, S. M., and Krause, J. E. (2005). Letter re: melanin-concentrating hormone and energy balance. J. Clin. Endocrinol. Metab. 90:6337. doi: 10.1210/jc.20051367

Whiddon, B. B., and Palmiter, R. D. (2013). Ablation of neurons expressing melanin-concentrating hormone $(\mathrm{MCH})$ in adult mice improves glucose tolerance independent of MCH signaling. J. Neurosci. 33, 2009-2016. doi: 10.1523/JNEUROSCI.3921-12.2013

Williamson-Hughes, P. S., Grove, K. L., and Smith, M. S. (2005). Melanin concentrating hormone $(\mathrm{MCH})$ : a novel neural pathway for regulation of $\mathrm{GnRH}$ neurons. Brain Res. 1041, 117-124. doi: 10.1016/j.brainres.2004.11.066

Willie, J. T., Sinton, C. M., Maratos-Flier, E., and Yanagisawa, M. (2008). Abnormal response of melanin-concentrating hormone deficient mice to fasting: hyperactivity and rapid eye movement sleep suppression. Neuroscience 156, 819-829. doi: 10.1016/j.neuroscience.2008.08.048

Woods, S. C., Lotter, E. C., Mckay, L. D., and Porte, D. Jr. (1979). Chronic intracerebroventricular infusion of insulin reduces food intake and body weight of baboons. Nature 282, 503-505. doi: 10.1038/282503a0

Wu, M., Dumalska, I., Morozova, E., Van Den Pol, A., and Alreja, M. (2009). Melanin-concentrating hormone directly inhibits GnRH neurons and blocks kisspeptin activation, linking energy balance to reproduction. Proc. Natl. Acad. Sci. U.S.A. 106, 17217-17222. doi: 10.1073/pnas.0908200106

Xu, L., Bloem, B., Gaszner, B., Roubos, E. W., and Kozicz, T. (2009). Sexspecific effects of fasting on urocortin 1 , cocaine- and amphetamine-regulated transcript peptide and nesfatin-1 expression in the rat Edinger-Westphal nucleus. Neuroscience 162, 1141-1149. doi: 10.1016/j.neuroscience.2009. 05.003

Yang, S. C., and Shieh, K. R. (2005). Differential effects of melanin concentrating hormone on the central dopaminergic neurons induced by the cocaine- and amphetamine-regulated transcript peptide. J. Neurochem. 92, 637-646. doi: 10.1111/j.1471-4159.2004.02896.x

Yao, Y., Fu, L. Y., Zhang, X., and Van Den Pol, A. N. (2012). Vasopressin and oxytocin excite $\mathrm{MCH}$ neurons, but not other lateral hypothalamic GABA neurons. Am. J. Physiol. Regul. Integr. Comp. Physiol. 302, R815-R824. doi: 10.1152/ajpregu.00452.2011 
Zakrzewska, K. E., Cusin, I., Stricker-Krongrad, A., Boss, O., Ricquier, D., Jeanrenaud, B., et al. (1999). Induction of obesity and hyperleptinemia by central glucocorticoid infusion in the rat. Diabetes Metab. Res. Rev. 48, 365-370. doi: $10.2337 /$ diabetes.48.2.365

Zhang, X., and van den Pol, A. N. (2012). Thyrotropin-releasing hormone (TRH) inhibits melanin-concentrating hormone neurons: implications for TRH-mediated anorexic and arousal actions. J. Neurosci. 32, 3032-3043. doi: 10.1523/JNEUROSCI.5966-11.2012

Zhang, Y., Proenca, R., Maffei, M., Barone, M., Leopold, L., and Friedman, J. M. (1994). Positional cloning of the mouse obese gene and its human homologue. Nature 372, 425. doi: 10.1038/372425a0

Zola-Morgan, S., Squire, L. R., and Amaral, D. (1986). Human amnesia and the medial temporal region: enduring memory impairment following a bilateral lesion limited to field CA1 of the hippocampus. J. Neurosci. 6, 2950-2967.
Conflict of Interest Statement: The authors declare that the research was conducted in the absence of any commercial or financial relationships that could be construed as a potential conflict of interest.

The reviewer SEK and handling Editor declared their shared affiliation, and the handling Editor states that the process nevertheless met the standards of a fair and objective review.

Copyright ( 12017 Diniz and Bittencourt. This is an open-access article distributed under the terms of the Creative Commons Attribution License (CC BY). The use, distribution or reproduction in other forums is permitted, provided the original author(s) or licensor are credited and that the original publication in this journal is cited, in accordance with accepted academic practice. No use, distribution or reproduction is permitted which does not comply with these terms. 


\section{Chapter 4}

Litter size determines the number of melanin-concentrating hormone neurons in the medial preoptic area of Sprague-Dawley lactating dams

Ferreira JGP' , Duarte JCG ${ }^{1}$, Diniz GB and Bittencourt JC

'These authors contributed equally to this work

As published in: Physiology and Behavior 181 75-79. 2017.

DOI: 10.1016/j.physbeh.2017.08.028 



\section{Litter size determines the number of melanin-concentrating hormone neurons in the medial preoptic area of Sprague Dawley lactating dams}

\section{A R T I C L E I N F O}

\section{Keywords:}

Maternal behavior

Lactation

Neuropeptide

Hypothalamus

$\mathrm{MCH}$

\begin{abstract}
A B S T R A C T
Melanin-concentrating hormone $[\mathrm{MCH}]$ is an important neuromodulator related to motivated behaviors. The $\mathrm{MCH}$-containing neurons are mainly located in the lateral hypothalamic area, zona incerta, and incerto-hypothalamic area. In the medial preoptic area [MPOA], a key region for the regulation of maternal behavior, Pmch mRNA expression and MCH synthesis can be detected exclusively during the lactation period. As litter size affects different parameters of maternal physiology, the aim of this study was to verify whether litter size can modulate the number of MCH-containing neurons in the MPOA of lactating rats. The dams were divided into the following groups: postpartum day 12,15, or 19, with a large, small or reduced litter. Our results show that the number of MCH-immunoreactive neurons in the MPOA is positively correlated with the number of pups in the litter and that artificially reducing the number of pups can also decrease the number of $\mathrm{MCH}$-immunoreactive neurons in the MPOA.
\end{abstract}

\section{Introduction}

Melanin-concentrating hormone $[\mathrm{MCH}]$ is an important neuropeptide implicated in the modulation of motivated behaviors $[1,2]$. The MCH-containing neurons are mainly located in the lateral hypothalamic area [LHA], zona incerta [ZI] and incerto-hypothalamic area [IHy] in both male and female brains [3-7]. An additional group of immunoreactive neurons can be detected in the medial preoptic area [MPOA] of lactating females, while this synthesis does not occur during gestation, post-weaning or any phase of the estrous cycle $[8,9]$. The MPOA is regarded as an important regulatory hub in the maternal behavior network, since lesions in this area impair such behavior [10]. Previous studies have shown Pmch mRNA expression and MCH synthesis in the MPOA progressively increase until the 15th day of lactation and are sustained at high levels until the 19th day, at which time they steeply decrease $[8,9,11]$.

Recent studies have implicated $\mathrm{MCH}$ in the maternal behavioral response to pups. Alachkar et al. [12] showed that $\mathrm{Pmch}^{-/-}$mice display impaired maternal behavior, while Benedetto et al. [13] demonstrated that MCH injections into the MPOA reduce active components of maternal behavior, including retrieval and nest building. These works suggest that $\mathrm{MCH}$ is necessary for the correct display of maternal behavior in the postpartum period and may act as an inhibitor of maternal behavior at later stages to start and/or facilitate the weaning process.

Moreover, it has been shown that, during lactation, the litter size can affect different parameters of maternal physiology, mainly due to the stimulus of suckling [14,15]. The lack of information about the factors that can modulate MCH synthesis in the MPOA led to this study, which aimed to verify whether the litter size can modulate the number of MCH-containing neurons in the MPOA.

\section{Materials and methods}

\subsection{Animals}

Adult female [80-90 days old] Sprague Dawley rats were housed four per cage in a light- and temperature-controlled environment [12-h light/12-h dark cycle, $21 \pm 2{ }^{\circ} \mathrm{C}$ ], with rat chow and water available ad libitum. After the regularity of the estrous cycle was confirmed through the vaginal cytology method, two females were housed with one male for breeding. Approximately 15 days after mating, the females

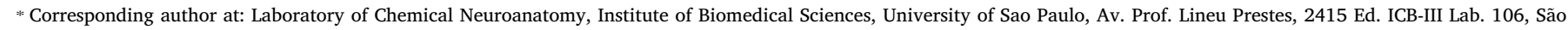
Paulo, SP 05508-000, Brazil.

E-mail address: jcbitten@icb.usp.br (J.C. Bittencourt).

${ }^{1}$ These authors contributed equally to this work. 

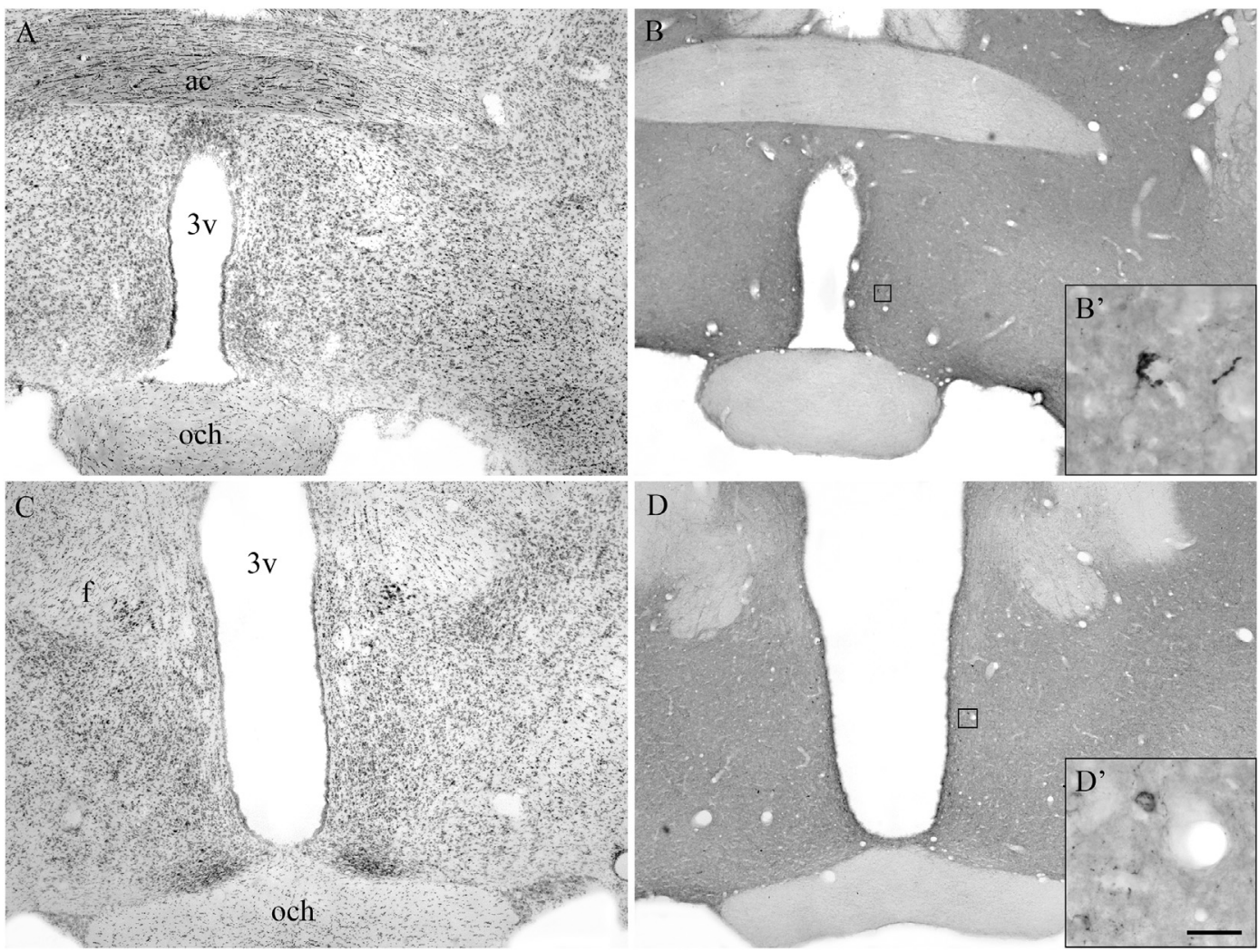

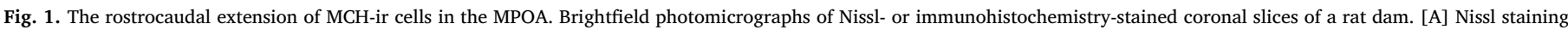

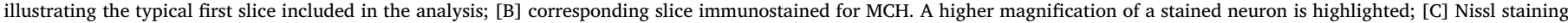

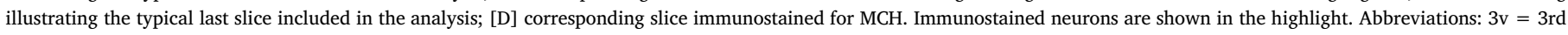
ventricle; $a c=$ anterior commissure; $f=$ fornix; och $=$ optic chiasm. Scale bar: $250 \mu \mathrm{m}$ in A-D; $50 \mu \mathrm{m}$ in B' and $\mathrm{D}^{\prime}$.
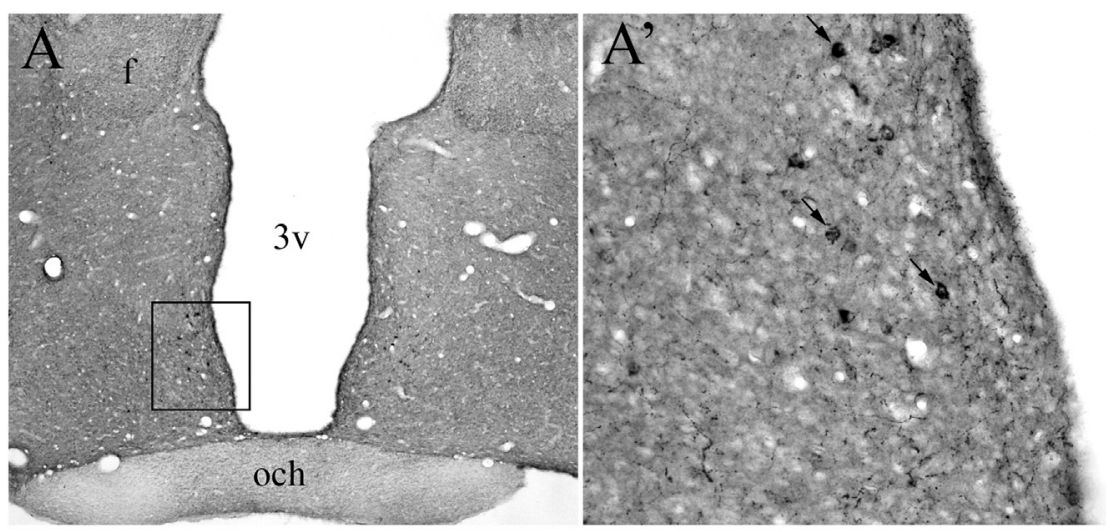

Fig. 2. Localization of MCH-ir cells in the MPOA on postpartum day 19 with different litter sizes. Brightfield photomicrographs show a small amount of $\mathrm{MCH}$-ir [black arrows] in the group with small litter size [6 pups; A, $\mathrm{A}^{\prime}$ ] compared with a large litter size [9 pups; B, B']. $\mathrm{A}^{\prime}$ and $\mathrm{B}^{\prime}$, higher magnification of $\mathrm{A}$ and $\mathrm{B}$, respectively. $3 \mathrm{v}=3 \mathrm{rd}$ ventricle; $\mathrm{f}=$ fornix; och $=$ optic chiasm. Scale bar: $250 \mu \mathrm{m}$ in $\mathrm{A}$ and $\mathrm{B} ; 50 \mu \mathrm{m}$ in $\mathrm{A}^{\prime}$ and $\mathrm{B}^{\prime}$.
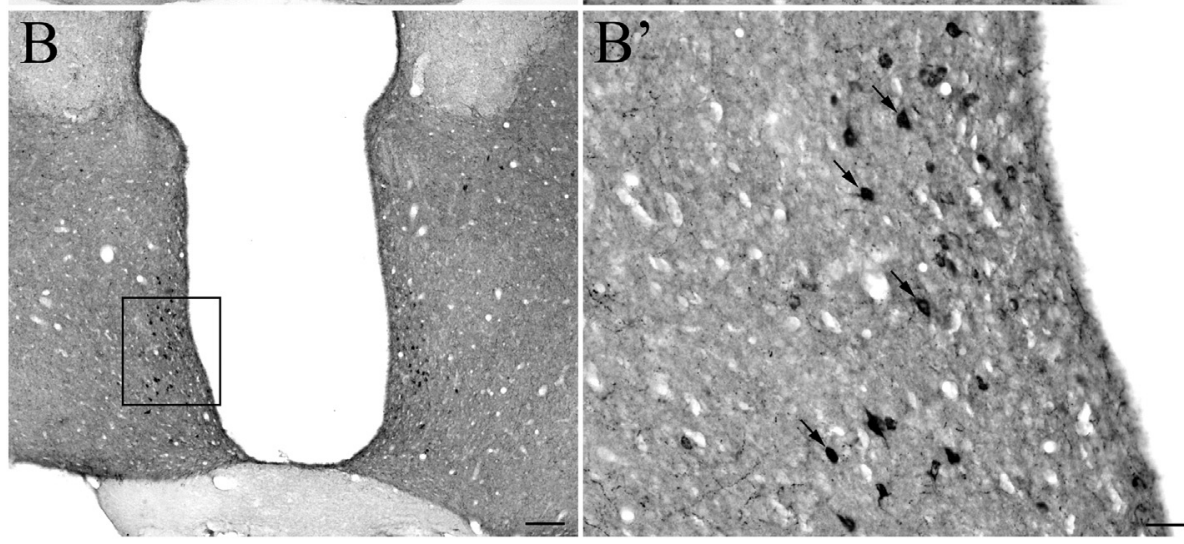


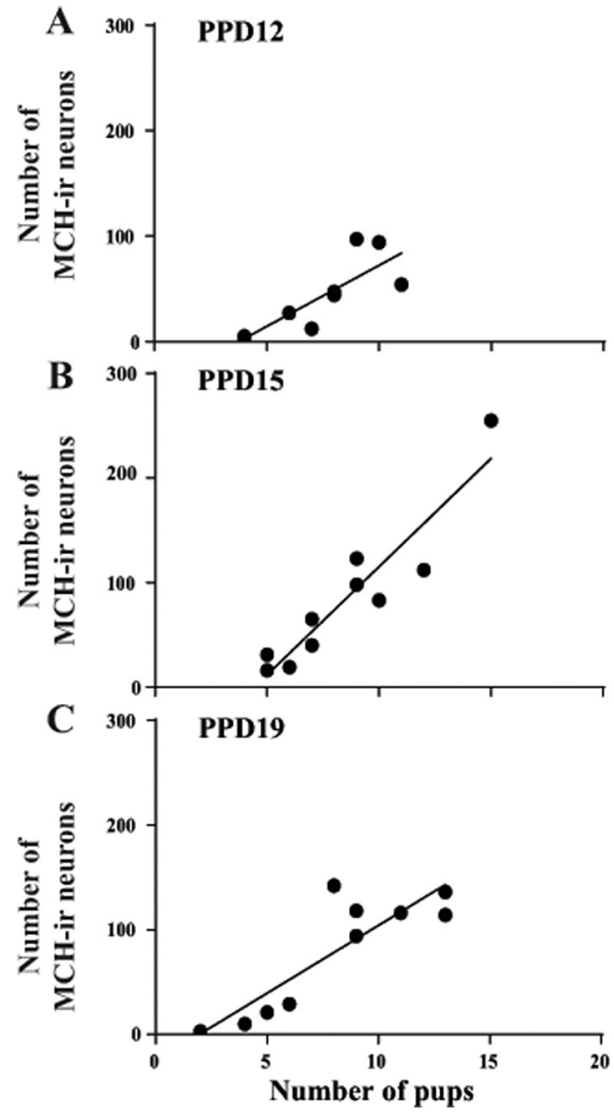

Fig. 3. Scatter plots and trend lines reveal the positive relationships between the numbers of pups and the MCH-ir neurons in the MPOA. A: PPD12 $\left[r=0.8107 ; r^{2}=0.6572\right]$; B: PPD15 [ $\left.r=0.9314 ; r^{2}=0.8676\right]$; C: PPD19 $\left[r=0.8691 ; r^{2}=0.7553\right]$.

were isolated in individual cages. The experiments were carried out following the guidelines established by the Institute of Biomedical Sciences Animal Welfare Committee [protocol number 031/2015].

The day of parturition was considered postpartum day 0 [PPD0], and the dams were randomly assigned to three different groups depending on the day of euthanasia: postpartum days 12, 15, and 19 [PPD12, PPD15 and PPD19, respectively]. The numbers of male and female pups in each litter were recorded. A litter size with 8 or more pups was considered large, and one with fewer than 8 pups, small. An additional group of dams who had large litters $[\geq 8$ pups] reduced to small litters [4 pups] on PPD1 was euthanized on PPD19.

On the respective days, the dams were deeply anesthetized with an intraperitoneal injection of ketamine/xylazine [ketamine $90 \mathrm{mg} / \mathrm{kg}$ and xylazine $12 \mathrm{mg} / \mathrm{kg}$ ] and transcardially perfused with cold $0.9 \%$ saline followed by $4 \%$ formaldehyde [from paraformaldehyde heated at $\left.60-65{ }^{\circ} \mathrm{C}\right]$ in sodium tetraborate buffer $\left[\mathrm{pH} 9.5\right.$ at $4{ }^{\circ} \mathrm{C}, 700 \mathrm{~mL}$ over $25 \mathrm{~min}$ ]. Brains were removed, post-fixed in the same fixative for $4 \mathrm{~h}$ and cryoprotected overnight at $4{ }^{\circ} \mathrm{C}$ in $0.1 \mathrm{M}$ phosphate-buffered saline [PBS], pH 7.4, containing 20\% sucrose. The brains were sectioned in the frontal plane at a thickness of $40 \mu \mathrm{m}$ on a freezing microtome, and every fifth section was used for analysis. The series were collected in anti-freezing solution [20\% glycerol, $30 \%$ ethylene glycol in PBS] and stored at $-20^{\circ} \mathrm{C}$.

\subsection{Immunohistochemistry}

To detect the MCH neurons in the MPOA, we performed immunohistochemistry in one series of hypothalamic sections per dam in all groups. Free-floating sections were blocked with $2 \%$ normal donkey serum [Jackson Laboratories] and incubated in rabbit anti-MCH primary antiserum [1:70,000, kindly provided by Dr. Joan Vaughan and

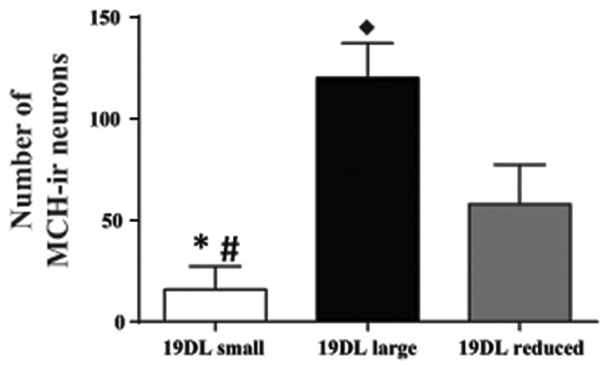

Fig. 4. Effect of litter size on MCH-ir in the MPOA. The number of MCH-ir neurons can be modulated by the number of pups in the litter. There were differences between small and large litter size [ $\left.{ }^{*} p<0.0001\right]$, between small and reduced litter size [\#p=0.0102], and between large and reduced litter size [ $\bullet p=0.0007]$.

Dr. Paul E. Sawchenko, Peptide Biology Laboratory, The Salk Institute, La Jolla, CA, USA] and $0.3 \%$ Triton X-100 overnight $[\approx 18 \mathrm{~h}]$ at room temperature. The antibody specificity was previously described by Bittencourt et al. [3]. This step was followed by incubation for $1 \mathrm{~h}$ in biotin-conjugated donkey anti-rabbit IgG [1:1000, Jackson Laboratories] and $1 \mathrm{~h}$ in avidin-biotin-HRP complex [1:500, Vector Labs, Burlingame, CA, USA]. The tissue was then subjected to a chromogen deposition reaction using 3,3'-diaminobenzidine tetrahydrochloride [DAB; Sigma], nickel sulfate [Fisher Scientific] and 0.03\% hydrogen peroxide in $0.2 \mathrm{M}$ sodium acetate buffer, $\mathrm{pH} 6.5$, for $2-3 \mathrm{~min}$. The reaction was terminated with rinses in sodium acetate buffer and then in potassium PBS [KPBS]. The sections were mounted onto gelatin-coated slides, dried overnight, dehydrated in graded ethanol, cleared in xylene and coverslipped with DPX.

\subsection{Data analysis and production of photomicrographs}

The results were analyzed using a Leica DMR microscope [Leica Microsystems, Wetzlar, Germany] under brightfield illumination. The number of $\mathrm{MCH}$-immunoreactive [MCH-ir] neurons in the MPOA of both hemispheres was counted. To avoid slicing angle discrepancies, we included six slices from each animal in the analysis, ranging approximately from -0.1 to $-1.1 \mathrm{~mm}$ relative to bregma (Fig. 1 ; [16]), and the total number of neurons was utilized in the analyses. These counts were carried out manually by two independent investigators blind to the group they were analyzing, and the values were compared and recorded. Occasional discrepancies in the total number of neurons were solved using the mean of the two investigators' counts.

Images were acquired using a microscope Nikon ECLIPSE 80i microscope connected to a Dell computer equipped with the Neurolucida Suite (MicroBrightField, Inc./MBF, Williston, VT, USA). Adobe Photoshop 13.0 image-editing software (Adobe Systems Inc., San Jose, CA, USA) was used to combine photomicrographs into plates. Only sharpness, contrast and brightness were adjusted.

\subsection{Statistical analysis}

Correlation analysis was performed using linear regression analysis, while mean differences as a group effect were evaluated through oneand two-way analysis of variance [ANOVA]. The normal distribution of samples was confirmed by the Shapiro-Wilk method, and the homoscedasticity of the samples was evaluated by the Brown-Forsythe method. A $p$-value $<0.05$ was considered statistically significant. All analyses were performed with GraphPad Prism 6.01 statistical software [GraphPad Software Inc., La Jolla, CA, USA].

\section{Results}

The following groups of dams were formed based on litter size: PPD12 small $=$ 4, 4, 6, 7 pups; PPD12 large = 8, 8, 9, 10, 11 pups; 
PPD15 small $=5$, 5, 6, 7, 7 pups; PPD15 large $=9,9,10,12,15$ pups; PPD19 small $=2$, 4, 5, 6, pups; PPD19 large $=8,9,9,11,13$, 13 pups. The number of MCH-ir neurons in the MPOA was affected by litter size [Fig. 2], as there was a positive correlation between the number of pups in the litter and the total observed number of immunoreactive neurons in the MPOA for all days of lactation analyzed - PPD12 [Fig. 3A; linear regression model, $\mathrm{F}_{1,7}=13.42, \quad p=0.008$ ], PPD15 [Fig. 3B; $\mathrm{F}_{1,8}=52.41, p<0.0001$ ] and PPD19 [Fig. 3C; $\mathrm{F}_{1,8}=24.69$, $p=0.0011]$. The two-way ANOVA revealed main effects of postpartum stage [PPD12, PPD15 and PPD19; $\left.\mathrm{F}_{2,22}=4.126, p=0.0301\right]$ and litter size [small and large; $\mathrm{F}_{1,22}=43.94, p<0.0001$ ].

To verify whether the prepartum or the postpartum litter size was responsible for the observed alterations in the number of immunoreactive neurons, we culled four large litters [8, 11, 12 and 13 pups] to 4 pups [2 males and 2 females] per litter on the first postpartum day and euthanized the dams at PPD19. There was a decrease in the number of MCH-ir neurons in the females with artificially small litters compared with females at the same day of lactation with naturally occurring large litters [Fig. 4; one-way ANOVA, $\mathrm{F}_{2,11}=49.61$, $p<0.0001]$.

\section{Discussion}

We demonstrated for the first time that the number of MCH-ir neurons in the MPOA is dependent on litter size. Multiple stimuli from the pups could account for this effect, including visual, tactile, olfactory and auditory cues [17]. The suckling stimulus is a particularly attractive candidate, as both prolactin concentration in the hypophysis [18] and the release of oxytocin and prolactin [19] are influenced by the suckling stimulus. Although hormonal action cannot be discounted, since several key alterations of the maternal physiology are precipitated by hormone alterations during pregnancy and parturition [20,21], our results suggest that the postpartum litter size is prevalent in determining the number of MCH-ir neurons in the MPOA.

Although the matter remains open to speculation, the action of litter size could be taken as indicative of the role of MPOA-located MCH-ir neurons in reproductive function. The expression of the KiSS-1 gene in the rat arcuate nucleus was decreased in dams that had a large litter size [10 or 15 pups] compared with dams that had 5 pups [14]. The kisspeptins are key regulator of the gonadal axis, controlling luteinizing hormone [LH] secretion [22]. Furthermore, RFRP-3, a type of RFamiderelated peptide also proposed as a modulator of the gonadal axis, had its gene expression increased in the dorsomedial nucleus of the hypothalamus when the litter size is large [23-25]. When taken together, these data suggest that litter size is especially relevant to the inhibition/reactivation of sexual function in dams. The $\mathrm{MCH}$ has been suggested to act on the gonadal axis through both gonadotropin-releasing hormone [GnRH] neurons and fibers [23] to modulate LH and follicle-stimulating hormone [FSH] release $[24,25]$. Therefore, $\mathrm{MCH}$ synthesis in the MPOA could represent a mechanism of reinstatement of sexual function in the weaning animal.

Finally, the litter-dependent synthesis of MCH in the MPOA may be linked to the termination of maternal behavior in preparation for weaning. The peak expression of MCH around PPD19 is contemporary with a decrease in maternal behavior [26], and the work of Benedetto et al. [13] suggests that MCH acts on the MPOA to decrease the active components of maternal behavior. Therefore, increased synthesis of MCH in the MPOA could be necessary to counteract the stronger and longer stimulation of maternal behavior expression by a larger litter, a mechanism that would guarantee that weaning occurred at the same time regardless of litter size. Further experimental work is necessary to confirm this hypothesis and expand our understanding of the role of $\mathrm{MCH}$ during the lactation period.

\section{Conclusions}

Our results indicate that a large litter size results in more $\mathrm{MCH}$-ir neurons in the MPOA of lactating dams and an artificially reduced litter size produces a decrease in the number of $\mathrm{MCH}$-ir neurons. These findings shed light on the interaction between dams and pups and help to clarify how the number of pups can modulate some features of the dam's brain, particularly in those structures related to maternal behavior.

\section{Acknowledgments}

JGPF was supported by the Fundação de Amparo à Pesquisa do Estado de São Paulo [FAPESP grant numbers 2015/05990-4 and 2016/ 19845-9]. GBD was supported by FAPESP [grant \#2016/02748-0]. JCB was supported by FAPESP [grants \#2010/52068-0 and 2016/02224-1]. We would also like to thank Coordenação de Aperfeiçoamento de Pessoal de Nível Superior [JCGD received a Master Student Fellowship from the Agency for the Advancement of Higher Education - CAPES, and JCB received grant \#848/15 from CAPES-COFECUB]. JCB is an Investigator with the Conselho Nacional de Desenvolvimento Científico e Tecnológico and is also supported by grant \#426378/2016-4 National Council for Scientific and Technological Development - CNPq.

\section{References}

[1] L.V. Sita, et al., Dopamine and melanin-concentrating hormone neurons are distinct populations in the rat rostromedial zona incerta, Brain Res. 970 (2003) 232-237.

[2] G.B. Diniz, J.C. Bittencourt, The melanin-concentrating hormone as an integrative peptide driving motivated behaviors, Front. Syst. Neurosci. 11 (2017).

[3] J.C. Bittencourt, et al., The melanin-concentrating hormone system of the rat brain: an immuno- and hybridization histochemical characterization, J. Comp. Neurol. 319 (1992) 218-245.

[4] L.V. Sita, et al., Connectivity pattern suggests that incerto-hypothalamic area belongs to the medial hypothalamic system, Neuroscience 148 (2007) 949-969.

[5] J.L. Bresson, et al., Human hypothalamic neuronal system revealed with a salmon melanin-concentrating hormone (MCH) antiserum, Neurosci. Lett. 102 (1989) 39-43.

[6] A. Viale, et al., Structure and expression of the variant melanin-concentrating hormone genes: only PMCHL1 is transcribed in the developing human brain and encodes a putative protein, Mol. Biol. Evol. 17 (2000) 1626-1640.

[7] C.F. Elias, et al., Characterization of CART neurons in the rat and human hypothalamus, J. Comp. Neurol. 432 (2001) 1-19.

[8] S. Knollema, et al., Novel hypothalamic and preoptic sites of prepro-melanin-concentrating hormone messenger ribonucleic acid and peptide expression in lactating rats, J. Neuroendocrinol. 4 (1992) 709-717.

[9] T.A. Rondini, et al., Chemical identity and connections of medial preoptic area neurons expressing melanin-concentrating hormone during lactation, J. Chem. Neuroanat. 39 (2010) 51-62.

[10] M. Numan, et al., Axon-sparing lesions of the preoptic region and substantia innominata disrupt maternal behavior in rats, Behav. Neurosci. 102 (1988) 381-396.

[11] R.D. Alvisi, et al., Suckling-induced Fos activation and melanin-concentrating hormone immunoreactivity during late lactation, Life Sci. 148 (2016) 241-246.

[12] A. Alachkar, et al., Inactivation of the melanin concentrating hormone system impairs maternal behavior, Eur. Neuropsychopharmacol. 26 (2016) 1826-1835.

[13] L. Benedetto, et al., Melanin-concentrating hormone in the medial preoptic area reduces active components of maternal behavior in rats, Peptides 58 (2014) 20-25.

[14] A. Noroozi, et al., Increased litter size and suckling intensity inhibit KiSS-1 mRNA expression in rat arcuate nucleus, Iran J. Basic Med. Sci. 17 (2014) 600-605.

[15] C. Lindblom, et al., Effects of pup sucking behaviour on inhibition of sexual behaviour and ovulatory secretion of LH in lactating rats, J. Endocrinol. 104 (1985) 419-425.

[16] L.W. Swanson, Structure of the Rat Brain, Elsevier, Amsterdam; London; London, 1992.

[17] R.B. Simerly, L.W. Swanson, The organization of neural inputs to the medial preoptic nucleus of the rat, J. Comp. Neurol. 246 (1986) 312-342.

[18] F. Mena, C.E. Grosvenor, Effect of number of pups upon suckling-induced fall in pituitary prolactin concentration and milk ejection in the rat, Endocrinology 82 (1968) 623-626.

[19] J.B. Wakerley, et al., Relationship between the suckling-induced release of oxytocin and prolactin in the urethane-anaesthetized lactating rat, J. Endocrinol. 76 (1978) 493-500.

[20] L. Keyser-Marcus, et al., Alterations of medial preoptic area neurons following pregnancy and pregnancy-like steroidal treatment in the rat, Brain Res. Bull. 55 (2001) 737-745.

[21] J.A. Russell, et al., Brain preparations for maternity - adaptive changes in behavioral and neuroendocrine systems during pregnancy and lactation. An overview, Prog. Brain Res. 133 (2001) 1-38. 
[22] J. Roa, M. Tena-Sempere, KiSS-1 system and reproduction: comparative aspects and roles in the control of female gonadotropic axis in mammals, Gen. Comp. Endocrinol. 153 (2007) 132-140.

[23] M.A. Johnson, et al., Rat RFamide-related peptide-3 stimulates GH secretion, inhibits LH secretion, and has variable effects on sex behavior in the adult male rat, Horm. Behav. 51 (2007) 171-180.
[24] M. Murakami, et al., Hypophysiotropic role of RFamide-related peptide-3 in the inhibition of LH secretion in female rats, J. Endocrinol. 199 (2008) 105-112.

[25] A. Noroozi, et al., Increased litter size and suckling intensity stimulate mRNA of RFamide-related peptide in rats, Int. J. Fertil. Steril. 9 (2015) 380-386.

[26] C.P. Cramer, et al., Weaning in rats: I. Maternal behavior, Dev. Psychobiol. 23 (1990) 479-493. 



\section{Chapter 5}

\section{Suckling-induced Fos activation and}

melanin-concentrating hormone immunoreactivity during late lactation

Alvisi RD', Diniz GB1, Da-Silva JM, Bittencourt JC
and Felicio LF
'These authors contributed equally to this work

As published in: Life Sciences 148 241-246. 2016. DOI: 10.1016/j.Ifs.2016.02.038 



\title{
Suckling-induced Fos activation and melanin-concentrating hormone immunoreactivity during late lactation
}

\author{
R.D. Alvisi ${ }^{\text {a,1 }}$, G.B. Diniz ${ }^{\text {b,1 }}$, J.M. Da-Silva ${ }^{\text {b }}$, J.C. Bittencourt ${ }^{\text {b,c }}$, L.F. Felicio ${ }^{\text {a,* }}$ \\ a Department of Pathology, School of Veterinary Medicine, University of São Paulo, São Paulo 05508-270, Brazil \\ b Laboratory of Chemical Neuroanatomy, Department of Anatomy, Institute of Biomedical Sciences, University of São Paulo, São Paulo 05508-000, Brazil \\ c Center for Neuroscience and Behavior, Institute of Psychology, University of São Paulo, Sao Paulo 05508-030, Brazil
}

\section{A R T I C L E I N F O}

\section{Article history:}

Received 3 September 2015

Received in revised form 6 February 2016

Accepted 9 February 2016

Available online 10 February 2016

\section{Keywords:}

Suckling stimulus

Maternal behavior

Fos

Medial preoptic area

Lateral hypothalamic area

Incerto-hypothalamic area

\begin{abstract}
A B S T R A C T
Aims: Melanin-concentrating hormone (MCH) is implicated in the control of food intake, body weight regulation and energy homeostasis. Lactation is an important physiological model to study the hypothalamic integration of peripheral sensory signals, such as suckling stimuli and those related to energy balance. MCH can be detected in the medial preoptic area (MPOA), especially around the 19th day of lactation, when this hormone is described as displaying a peak synthesis followed by a decrease after weaning. The physiological significance of this phenomenon is unclear. Therefore, we aimed to investigate hypothalamic changes associated to sensory stimulation by the litter, in special its influence over MCH synthesis.

Main methods: Female Wistar rats $(n=56)$ were euthanized everyday from lactation days $15-21$, with or without suckling stimulus (WS and NS groups, respectively). MCH and Fos immunoreactivity were evaluated in the MPOA and lateral and incerto-hypothalamic areas (LHA and IHy).

Key findings: Suckling stimulus induced Fos synthesis in all regions studied. An increase on the number of suckling-induced Fos-ir neurons could be detected in the LHA after the 18th day. Conversely, the amount of $\mathrm{MCH}$ decreased in the MPOA from days 15-21, independent of suckling stimulation. No colocalization between $\mathrm{MCH}$ and Fos could be detected in any region analyzed.

Significance: Suckling stimulus is capable of stimulating hypothalamic regions not linked to maternal behavior, possibly to mediate energy balance aspects of lactation. Although dams are hyperphagic before weaning, this behavioral change does not appear to be mediated by $\mathrm{MCH}$.
\end{abstract}

(C) 2016 Published by Elsevier Inc.

\section{Introduction}

Melanin-concentrating hormone $(\mathrm{MCH})$ is a nonadecapeptide that was first discovered in the fish class Teleostei and later described in mammals [1-4]. In the mammalian brain, $\mathrm{MCH}$ is synthesized mainly in cell bodies of hypothalamic sites such as in the lateral hypothalamic area (LHA), perifornical area and, to a lesser degree, in the incertohypothalamic area (IHy) [5]. Exclusively during the lactation period, MCH synthesis can be detected in neurons of the medial preoptic area, raising from the 5 th to the 19th day and decreasing concomitantly to the weaning of the pups $[6,7]$. The exact role played by $\mathrm{MCH}$ in physiological alterations of motherhood is still unclear. The projections of $\mathrm{MCH}$-immunoreactive [MCH-ir] neurons are broad, reaching for instance the median eminence, hippocampal formation, prefrontal cortex, periaqueductal gray matter, lateral part of the medial mammillary nucleus, nucleus accumbens, and medial septal nucleus. Thus, LHA and

\footnotetext{
* Corresponding author at: Department of Pathology, School of Veterinary Medicine, University of São Paulo, São Paulo ZIP:05508-900, Brazil.

E-mail address: lfelicio@usp.br (L.F. Felicio).

1 These authors contributed equally to this work.
}

IHy can be considered integrative centers that are optimally positioned to influence a wide range of systems and functions, not simply as direct modulators of effector and motor functions [8]. Melanin-concentrating hormone is involved in the regulation of body weight, acting on Gprotein-coupled receptors similarly to other central and peripheral peptides, such as orexin, cholecystokinin, and bombesin [9-11]. Additionally, intra-medial preoptic area injections of $\mathrm{MCH}$ altered monoamine concentrations in this area and stimulated sexual behavior and luteinizing hormone secretion, as well as a decrease in appetitive components of maternal behavior of dams [12-14]. The present study evaluated the daily influence of the suckling stimulus on $\mathrm{MCH}$ and Fos immunoreactivity (Fos-ir) in the LHA, MPOA, and IHy during late lactation.

\section{Materials and methods}

\subsection{Animals}

Adult Wistar rats $(n=56)$ were mated with approximately 90 days old at the beginning of the experiments. The rats were obtained from the Department of Pathology, School of Veterinary 
Medicine, University of São Paulo, and housed in polypropylene cages $(30 \mathrm{~cm} \times 40 \mathrm{~cm} \times 18 \mathrm{~cm}$ ) with approximately 1.01 of medium-grade pine flakes as bedding under a $12 \mathrm{~h} / 12 \mathrm{~h}$ light/dark cycle (lights on at 6:00 AM) with controlled temperature $(23 \pm$ $2{ }^{\circ} \mathrm{C}$ ). Rat chow and water were available ad libitum throughout the experiment. The animal housing and procedures were in accordance with the guidelines of the Committee on Care and Use of Animal Resources, School of Veterinary Medicine, University of São Paulo (proc. no. 2185/2011). These guidelines are similar to those of the National Research Council, USA.

\subsection{Mating}

At the end of the light period, female rats that were in the period of transition from proestrus to estrus were placed in the cage of a sexually experienced male. The day that sperm was observed in the vaginal lavage of each female was designated day 1 of pregnancy. On the second day after birth, the litters were culled to eight pups each (four females and four males).

\subsection{Experimental design}

The eight pups were left with the mother and the lactation progressed without interference until the weaning period, when animals were separated into two groups: by presence or not of suckling stimulus ( $n=28$ for each group - NS, no suckling - WS, normally exposed to suckling pups). Among those groups quoted above, again the animals were divided into subgroups, now by the day of sacrifice ( $n=4$ for each subgroup, again NS and WS) on the 15th to 21 st day of lactation. To create the NS group, we employed the vibrissae pad anesthesia technique employed by other authors $[15,16]$. Pups were separated from the mother $1 \mathrm{~h}$ before the test, and only in the NS group the pups received a topic cream of 5\% lidocaine (Xylocaína $50 \mathrm{mg} / \mathrm{g}$ - Astra Zeneca; London, United Kingdom) in their vibrissae pads, resulting in complete loss of vibrissal reflexes. They were returned to the mother and their capability to attach to the nipples was assessed. In cases where pups in the NS group were able to attach to the nipples, the mothers have been discontinued from the experiment. In both groups there was no impairment in the pups' capability to vocalize or in the mother retrieval, grooming and nest building. After $20 \mathrm{~min}$, pups were again separated from the mother and after $90 \mathrm{~min}-$ time to allow Fos synthesis - the dams were perfused. Tissue was then subjected to double immunohistochemical staining to identify regions of Fos- and MCH-ir. (See Fig 1.)

\subsection{Immunohistochemistry for double-labeling hypothalamic neurons with $\mathrm{MCH}$ and Fos}

All of the animals $(n=56)$ that were used in the experiments above were anesthetized with a lethal dose of $35 \%$ chloral hydrate ( $1 \mathrm{ml}$, i.p.) and perfused via the ascending aorta first with cold $0.9 \%$ saline ( $100 \mathrm{ml}$ for $\sim 1 \mathrm{~min}$ ), followed by $4 \%$ formaldehyde fixative (from paraformaldehyde heated to $60-65{ }^{\circ} \mathrm{C}$ ) in a borate buffer (pH 9.5) at $4{ }^{\circ} \mathrm{C}$ (800-1000 $\mathrm{ml}$ for approximately $25 \mathrm{~min}$ ). Immediately after perfusion, the brains were removed, postfixed for $2 \mathrm{~h}$ in the same fixative (supplemented with $20 \%$ sucrose), and transferred to $20 \%$ sucrose in $0.02 \mathrm{M}$ potassium phosphate-buffered saline (KPBS). A series of $30-\mu \mathrm{m}$ coronal sections (1:5 series) were cut on a freezing microtome and stored at $-20{ }^{\circ} \mathrm{C}$ in antifreeze solution.
To map MCH and Fos immunoreactivity and possible colocalization between these two markers, we first performed immunohistochemistry to label Fos-ir cells. One free-floating series of sections from each animal was pretreated with $0.3 \%$ hydrogen peroxide (Sigma Chemical, St. Louis, MO, USA) for $15 \mathrm{~min}$, followed by $2 \times 10 \mathrm{~min}$ rinses in KPBS and incubation in primary antibody against rabbit and anti-Fos (1:70,000; Millipore, catalog \# ABE 457 anti-c-Fos rabbit antibody polyclonal; for anti-Fos protocol vide [17]) in $3 \%$ normal goat serum diluted in KPBS $+0.3 \%$ Triton X-100, for $\sim 24$ h at room temperature. Subsequently, the sections were rinsed in KPBS $(2 \times 10 \mathrm{~min})$ and incubated for $1 \mathrm{~h}$ at room temperature in biotinylated goat anti-rabbit immunoglobulin G (IgG; Vector Laboratories, Burlingame, CA, USA) diluted 1:800 in KPBS with $0.3 \%$ Triton X100 , then for $1 \mathrm{~h}$ at room temperature using peroxidase avidinbiotin complex solutions (Vector) diluted 1:333 in KPBS. The sections were then rinsed and subjected to immunoperoxidase reactions using 3,3'-diaminobenzidine tetrahydrochloride (DAB; $0.02 \%$; Sigma) with $0.003 \%$ hydrogen peroxide and nickel ammonium sulfate (NAS) at $2.5 \%$ dilute in a sodium acetate buffer $0.2 \mathrm{M}$, pH 6.5. After $\sim 10 \mathrm{~min}$, the reaction was terminated with successive rinses in KPBS. Afterward, the sections were subjected to the second immunohistochemistry protocol to label MCH cells. The sections were incubated in anti-MCH (1:70,000; PBL \#234, kindly provided by Dr. J. Vaughan and Dr. Paul E. Sawchenko, Peptide Biology Laboratory, The Salk Institute, La Jolla, CA, USA). The specificity of the antibody was tested in previous studies [3,5]. The antibody was diluted in $3 \%$ normal goat serum in KPBS $+0.3 \%$ Triton X-100 for $\sim 24$ h at room temperature. Subsequently, the sections were rinsed in KPBS $(2 \times 10 \mathrm{~min})$ and incubated for $1 \mathrm{~h}$ at room temperature in biotinylated goat anti-rabbit IgG (Vector Laboratories, Burlingame, CA, USA) diluted $1: 800$ in KPBS with $0.3 \%$ Triton $X-100$, then for $1 \mathrm{~h}$ at room temperature using peroxidase avidin-biotin complex solutions (Vector) diluted 1:333 in KPBS. The sections were then rinsed and subjected to immunoperoxidase reaction using 0.02\% DAB (Sigma) with $0.003 \%$ hydrogen peroxide (but without NAS) diluted in a $0.2 \mathrm{M}$ sodium acetate buffer $(\mathrm{pH} 6.5)$. After $\sim 5 \mathrm{~min}$, the reaction was terminated with successive rinses in KPBS. The sections were mounted on gelatin-coated slides, dehydrated, delipidated, and coverslipped with a DPX mounting medium. An intervening series was subjected to thionin staining for reference using the Nissl method to delineate the cytoarchitecture of the surrounding regions.

\subsection{Stereology}

Stereology was conducted by using a modified optical fractionator probe and stereological image analysis software (Stereo Investigator, MBF Bioscience, USA) operating a computer-driven stage motorized in the $x, y$ and $z$ axes (Ludl Electronic Products; Hawthorne, NY, USA) of an Eclipse 80i microscope (Nikon Instruments, Tokyo, Japan) equipped for brightfield and darkfield imaging. Using a low power magnifying lens, the areas to be counted were traced and the counting frames distributed in an unbiased, systematic and random way by the analysis software. Stained cells were counted using a $40 \times$ objective ( $0.95 \mathrm{NA}$ ).

To ensure homogeneity among different animals, the areas to be counted were drawn always following the same rules: the LHA is defined as the quadrangle formed by a superior horizontal line that passes at the top of the third ventricle $(3 \mathrm{~V})$, an inferior horizontal line that crosses the fornix, a medial vertical line that also crosses at the fornix and a lateral line that follows the medial border of the internal capsule.

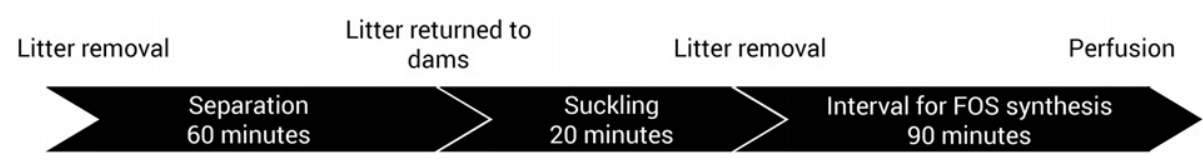


The upper and bottom limits of the IHy were drawn as two horizontal lines, one crossing the middle of the mammillothalamic tract (mt) and on the top of the $3 \mathrm{~V}$. The medial limit was also the $3 \mathrm{~V}$ and the lateral limit the mt. Finally, the MPOA was limited by two horizontal lines at the optic chiasm and the anterior commissure and the lateral limit was drawn as a line over the lateral-most point of the optic chiasm.

For each region, exactly 3 slices per animal were counted. Although there are more LHA-containing slices anteriorly than we have counted, the copresence with the IHy could have led to miscounts between the two regions. Therefore, we decided to evaluate the LHA after the posterior end of the IHy. Setting the same number of slices counted allowed us to exclude possible variations of area length due biased slicing of the samples.

\subsection{Statistical analysis}

The data were square-root transformed and subjected to two-way ANOVA, with the groups (WS/NS) and days of lactation (days 15-21) as factors, followed by Tukey or Šídák multiple-comparison tests as appropriate. The level of significance was $p<0.05$. The results are expressed as mean \pm standard error of the mean (SEM). In all cases the individual values of each animal were used for the statistical analysis. The means of the groups were used to calculate the Pearson correlation coefficient between the total number of MCH-ir neurons and both total number of Fos-ir neurons and the number of neurons activated by the suckling stimulus. All calculation was performed using Prism 6 (GraphPad, San Diego, CA, USA).

\section{Results}

Fos immunoreactivity was significantly different between WS and NS groups, independent of the day of lactation, in all areas analyzed (analysis of variance - ANOVA: LHA $-F_{1,42}=13.97$, $p<0.001$; MPOA $-F_{1,42}=11.94, p=0.001$; $\mathrm{IHy}-F_{1,42}=7.43$, $p=0.009$ ). In all cases, animals from the WS group displayed the highest values (Fig 2).

The number of $\mathrm{MCH}$-ir cells was affected by the day of lactation both in the MPOA as in the IHy. MCH-ir decreases in the MPOA as lactation progresses independent of suckling stimulus (ANOVA: $\mathrm{F}_{6,42}=4.81$, $p<0.001$ ), with the 20th and 21st days displaying less MCH-ir cells than the 15th and 16th days (Šídák: $p<0.05$ ). In the IHy there is an effect of the day of lactation (ANOVA: $\mathrm{F}_{6,42}=3.05, p=0.01$ ), with a decrease in the 19th day when compared to the 17th and 21st days (Tukey: $p<0.05$ ). For the number of MCH-ir cells in the LHA, the ANOVA test revealed an interaction effect between lactation day and suckling stimulus (ANOVA: $\mathrm{F}_{6,42}=2.57, p=0.03$ ) (Fig 2). The Pearson correlation factor was $<0.2$ for all regions analyzed, indicating that there is no linear relationship between Fos activation and MCH synthesis decrease.

In all cases analyzed, Fos-ir and MCH-ir cells formed distinct subpopulations, as no colocalization between these two proteins could be detected in any region. There was a notable proximity between these two populations, which may suggest that suckling-activated cells are capable of interfering with the MCH peptidergic system (Fig 3).

\section{Discussion}

In the present study we have evaluated the relationship between $\mathrm{MCH}$ and Fos immunoreactivity in key regions to the energy balance and maternal behavior [LHA, IHy and MPOA] in the later stages of lactation. We showed that Fos-ir in all areas analyzed is strongly linked to the suckling stimulus from the pups, even in the LHA and the IHy, regions that are not directly associated to maternal behavior.

This result is in general agreement to previous studies [18-20] that show an important increase in Fos synthesis after dams are exposed to their litters. In contrast to those works, though, that focused mainly on the early postpartum period, our study focused on the weaning pups, from the 15th to the 21st lactation days. As in this stage the intensity of maternal behavior diminishes [21] and the mother spends less time with the pups [22], it could be hypothesized that dams are less stimulated by pups. This is not the case. Mothers displayed a 3-fold increase in Fos-ir cells in the LHA from days 15-18 and a 10-fold increase in days 19-21. Similarly, stimulated dams displayed 2.5 times more Fos-ir cells in the MPOA and in the IHy than non-stimulated dams.

To interpret this results it is important to look at what is happening to the pups as well as to the mother at this point. As described by Thiels et al. [23], the transition of milk to solid food can be described in three phases: one that goes from days $14-18$, when the pups still rely mostly on maternal feeding; one that goes from days $18-24 / 25$, marked by the increasing independence of the pups from the mother; and a last stage that is characterized by the termination of milk feeding and the display of mature behavior by the pups. Our increase in Fos-ir due to suckling stimulation reflects the stages proposed by those authors. The onset of independent ingestion temporally correlates to an increase in Fos synthesis in the LHA, which could indicate that the mother becomes more sensitive to the suckling stimulus at the same time that the pups spend less time feeding on her. Concomitantly, the dams have an increase in food intake that starts at days 17-18 of lactation [24] and last until the pups are weaned. This indicates that orexigenic circuits are more easily elicited by the suckling stimulus after day 18 , possibly as a compensation mechanism for the reduced time the pups spend sucking. The fact that there was no colocalization of Fos and MCH in the LHA, however, indicates that the orexigenic circuits facilitated are not dependent on MCH activation. A similar increase in Fos activation could not be observed in the MPOA or the IHy, where the Fos-ir ratio of WS/NS animals is constant from days 15-21. A causal relationship between the increase of Fos synthesis and food consumption at days 18 onward is further supported by the classical role in energy homeostasis and food seeking associated to the LHA [25-27].

The exact role of the activated cells in the IHy is harder to predict, as this area has just recently been described and its functions are not clear. It was suggested that this area participates in the expression of reproductive and defensive behaviors [28]. Hansen and Ferreira [29] have shown that rats with peripeduncular area lesions (which block suckling-activated milk ejection) display decreased aggression toward intruders. Suckling-mediated activation of IHy cells during the lactation period could be a plausible mechanism to explain the heightened aggression of dams. As in the LHA, MCH neurons are not obviously stimulated by suckling, as no colocalization was found.

Finally, the MPOA also displayed Fos activation mediated by the suckling stimulus. Although numerous works also found Fos activation in the MPOA of maternal rats, only Lonstein et al. [16] tried to dissect the sucking stimulus from other modalities of sensorial input to which the female is exposed during motherhood. Comparing our work to Lonstein's, these researchers found no differences between sucklingstimulated and non-stimulated dams. This apparent contradiction may be explained by different experimental conditions in the two works. Lonstein et al. [16] deprived mothers from the pups for $48 \mathrm{~h}$ and evaluated dams in the early lactation period (lactation day 7), while we employed much shorter deprivation times $(1 \mathrm{~h})$ and examined the late stage of lactation. Both factors could make mothers more susceptible to other stimuli, masking the subtler effects on Fos activation of tactile stimulation. It is also possible that the divergence found reflects innate differences among strains, as it has been suggested before [30].

Although the role of the MPOA on the onset and maintenance of maternal behavior in the early stages of lactation has been thoroughly studied, and Fos activation of neurons in this area could point to a straightforward stimulating effect of suckling over maternal behavior, the time frame of this experiment complicates this interpretation. As pointed out by Pereira and Morrell [31], inhibiting the MPOA in the lactation day 5 produces a decline in maternal behavior, but when this inhibition is made in lactation day 14 the effect is reversed and there is an 
$\mathrm{MCH}$
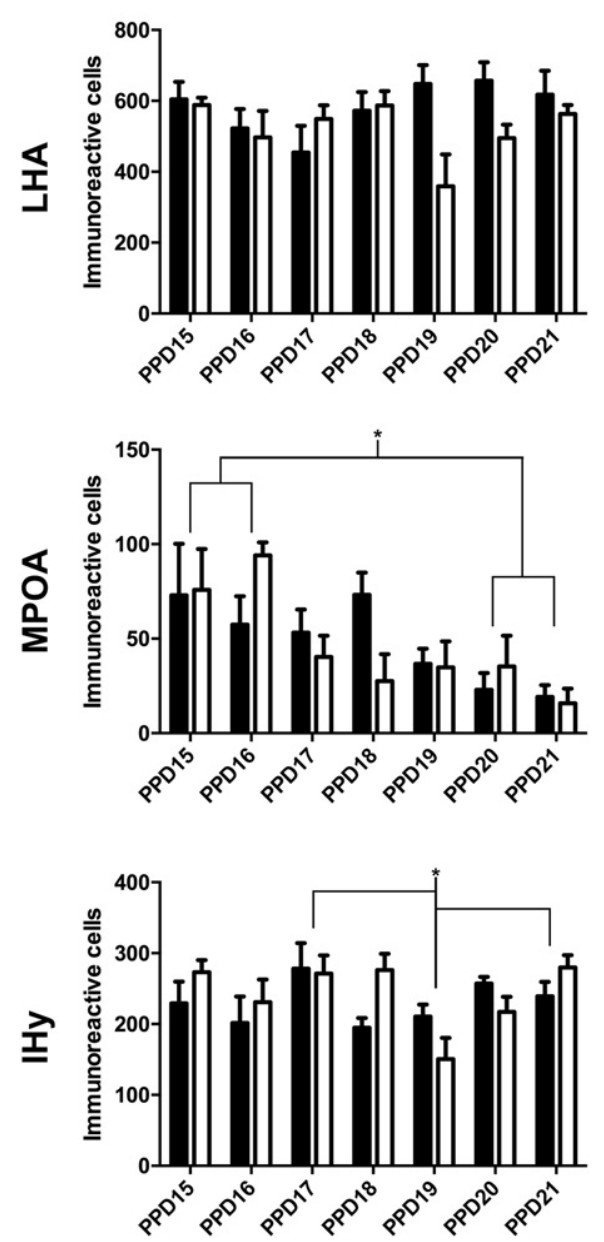

Fos
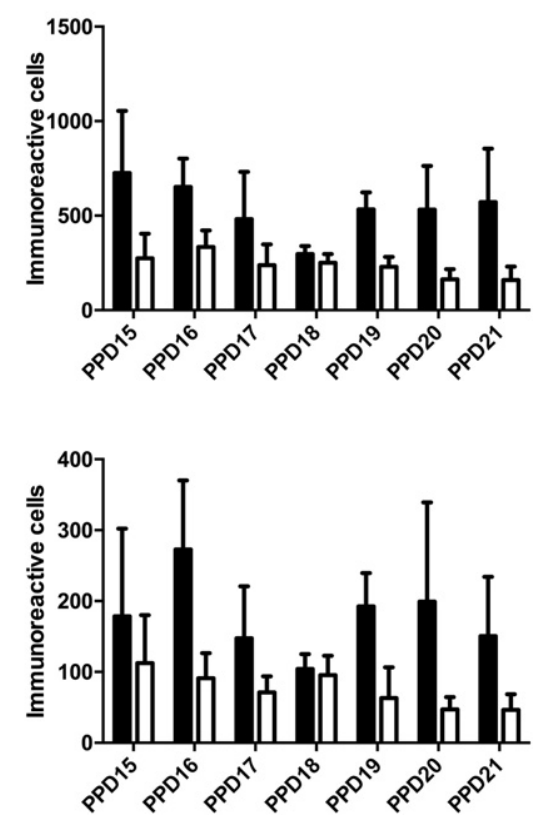

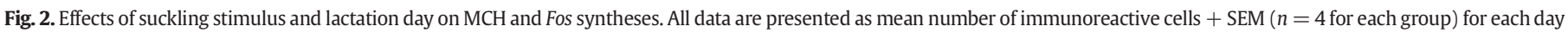
of lactation. Black bars represent dams of the WS group and white bars of the NS group. Statistical significance: ${ }^{*} p<0.05$.

increase in the care of the pups, which indicates that the MPOA acquires an inhibitory role as lactation progresses.

Interestingly, the time frame of MPOA switching is compatible to the synthesis of MCH in the MPOA. Different authors have reported that the production of $\mathrm{MCH}$ in the MPOA raises as lactation progresses, with more MCH-ir neurons at the 19th day when compared to the 12th day and a very discreet synthesis in the 5 th day $[6,7]$. In our work we observed that the amount of $\mathrm{MCH}$ decreases from the 15 th to the 21st day. If the number of MCH-ir neurons in the MPOA is higher in the 15th day than it is in the 19th, this also means that it is higher than in the 12th day, pointing to a steep increase happening between the 12 th and 15 th days of lactation. This increase in $\mathrm{MCH}$ synthesis correlates quite well to the results of Grota and Ader [21], that showed a decrease in nest building and retrieval that starts around the 13th day of lactation. Also in agreement to that is the work of Benedetto et al. [14], that shows that microinjections of $\mathrm{MCH}$ in the MPOA reduce active components of maternal behavior such as nest building and retrieval. The naturally occurring raise in $\mathrm{MCH}$ synthesis in the MPOA between days 12 and 15 of lactation could be the mechanism that acts to reduce maternal behavior and prepare the pups for weaning.

The microinjections made by Benedetto et al. [14] did not produce any effect on consummatory aspects of maternal behavior as time spent nursing. Likewise, the decline in nursing appears to start before the raise of MCH synthesis in the MPOA, around the 9th day [21]. As the suckling stimulus is linked to the consummatory aspects of maternal behavior, it is possible that it has little to no effect on $\mathrm{MCH}$-ir neurons (no Fos/MCH colocalization) because $\mathrm{MCH}$ activation to decrease retrieval and nest building is derived from forebrain signals, normally associated to the appetitive components.

Although there is no direct colocalization between $\mathrm{MCH}$ synthesis and Fos activation, a relationship between these two cannot be discarded. First, MCH activation may be mediated by other transcription factors other than Fos, since its promoter region contains sequences for other transcription elements [32]. Second, it is possible that the Fos-ir populations in each area interact with $\mathrm{MCH}$ neurons to modulate their function, resulting in downstream effects. Lonstein et al. [33] have shown that almost half of all Fos-ir neurons that appear in the MPOA of dams following pup stimulation are ER $\alpha$-ir. Therefore, the increase in Fos-ir cells observed in the MPOA of WS dams could also reflect the activation of ER $\alpha$-ir neurons which may, in turn, interact with the MCH-ir population. A similar interaction may happen in all areas studied, since ER $\alpha$ neurons are present in the MPOA, LHA and possibly in the IHy (as suggested by its presence in the anterior hypothalamic area and zona incerta in works previous to the description of the IHy [34]). We are not the first to suggest such relationship, since Muschamp and Hull [35] propose that ER $\alpha$ neurons of the LHA modulate the MCH population, producing the anoretic effect observed in males resulting from estradiol injection. Another possibility is that, rather than $\mathrm{MCH}$, other orexigenic neuropeptide could be activated after suckling stimulus, such as orexin, in the LHA [36]. 

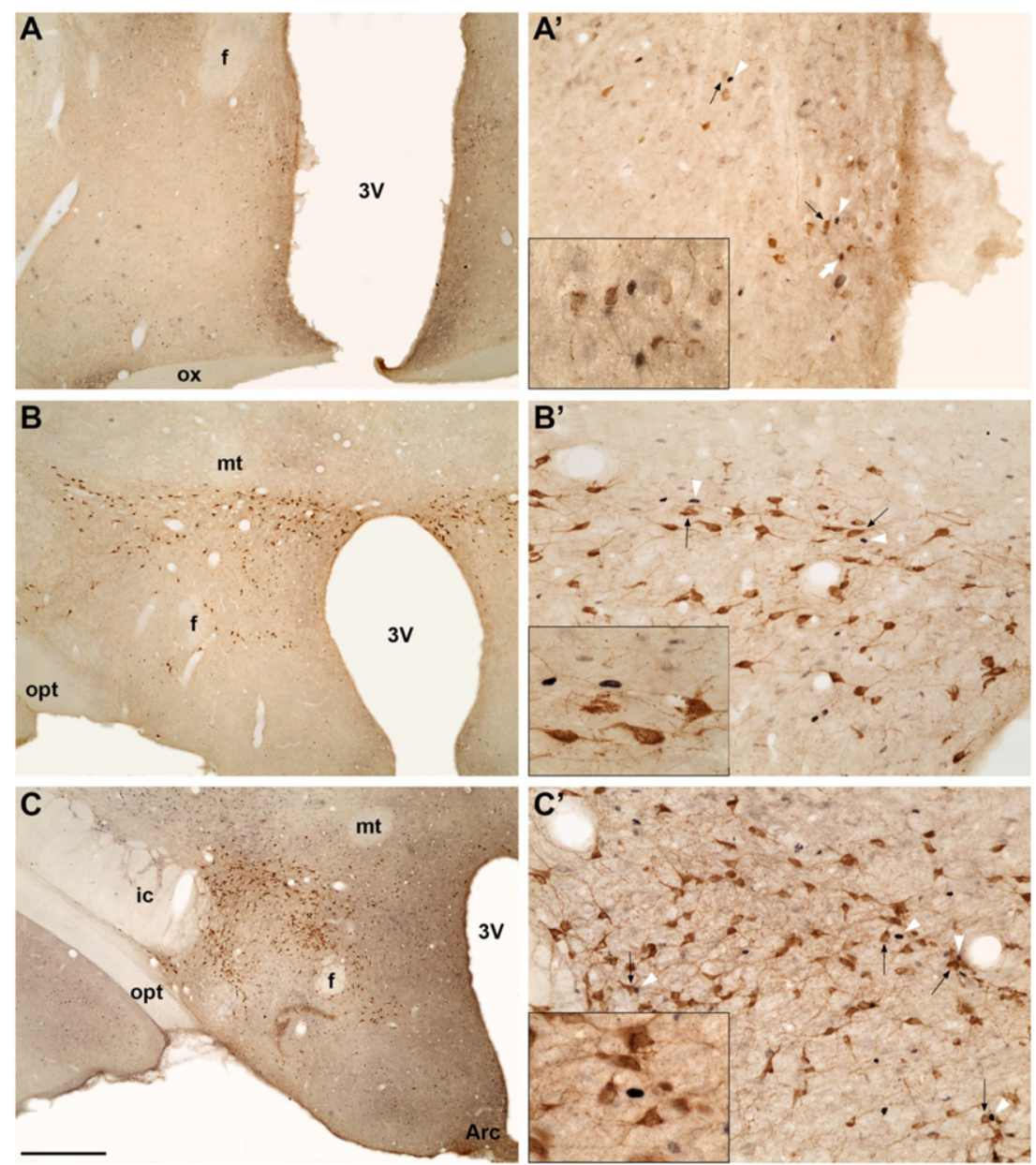

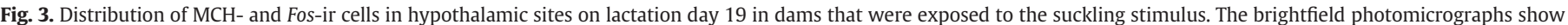

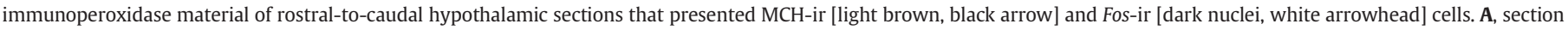

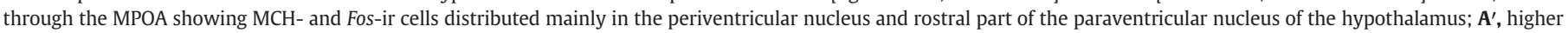

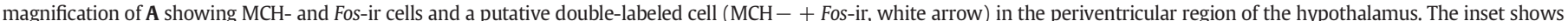

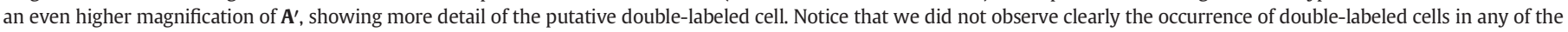

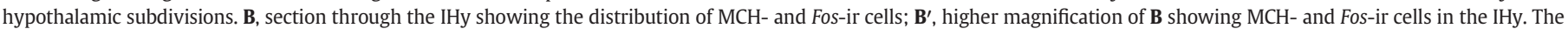

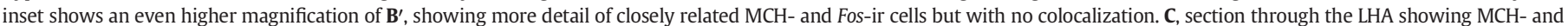

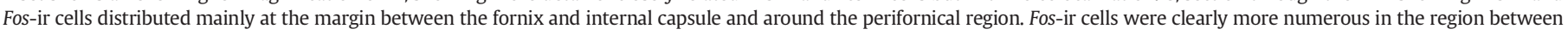

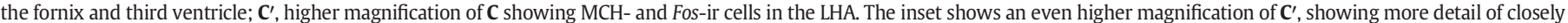
related MCH- and Fos-ir cells but no colocalization. Scale bars $=500 \mu \mathrm{m}$ in A, B, and C; $200 \mu \mathrm{m}$ in A', B', and C'; and $100 \mu \mathrm{m}$ in insets.

Placing together the suckling-mediated increase of Fos-ir cells in the LHA and the IHy, regions not directly associated to maternal behavior, this would indicate that the hypothalamic changes associated to mother-offspring interactions are more extensive and complex. Besides, areas not directly associated to maternal care could be important sites of inquiry to better understand the maternal brain. Further chemical characterization of activated neurons and time-sensitive experiments may provide important data regarding the dam physiology.

\section{Conclusions}

In the present study, $\mathrm{MCH}$ and Fos immunoreactivity were measured during late lactation in the LHA, MPOA, and IHy to investigate the sensory and endocrine factors that influence the expression of $\mathrm{MCH}$. The suckling stimulus elicited Fos synthesis not only in a region associated to maternal behavior (MPOA), but also in regions associated to energy homeostasis (LHA) and putative neuroendocrine control (IHy). This activation was not correlated to $\mathrm{MCH}$ function, as no colocalization was detected. The synthesis of $\mathrm{MCH}$ decreases as lactation progresses in the MPOA, but remains constant in the LHA. Areas where we detected Fos immunoreactivity may have stimulated $\mathrm{MCH}$-producing neurons, and an indirect relationship between the areas of neuronal activation and $\mathrm{MCH}$ expression seems likely during the suckling stimulus. Further chemical characterization of neurons is necessary to fully comprehend the extension of suckling-induced changes in the maternal brain.

\section{Conflict of interest statement}

The authors declare that there are no conflicts of interest.

\section{Acknowledgments}

We would like to thank Dr. Paul E. Sawchenko and Dr. Joan Vaughan from The Salk Institute for Biological Studies for the generous gift of the antiserum against $\mathrm{MCH}$. Financial support for this study was provided in the form of a grant from Fundação de Amparo à Pesquisa do Estado de São Paulo (FAPESP; grant no. 10/52068-0). We would also like to thank Coordenação de Aperfeiçoamento de Pessoal de Nível Superior (CAPES). J.C.B. is an Investigator with Conselho Nacional de Desenvolvimento Científico e Tecnológico (CNPq). 


\section{References}

[1] H. Kawauchi, et al., Characterization of melanin-concentrating hormone in chum salmon pituitaries, Nature 305 (5932) (1983) 321-323.

[2] T. Rance, B.I. Baker, The teleost melanin-concentrating hormone - a pituitary hormone of hypothalamic origin, Gen. Comp. Endocrinol. 37 (1) (1979) 64-73.

[3] J.L. Nahon, et al., The rat melanin-concentrating hormone messenger ribonucleic acid encodes multiple putative neuropeptides coexpressed in the dorsolateral hypothalamus, Endocrinology 125 (4) (1989) 2056-2065.

[4] J.M. Vaughan, et al., Characterization of melanin-concentrating hormone from rat hypothalamus, Endocrinology 125 (3) (1989) 1660-1665.

[5] J.C. Bittencourt, et al., The melanin-concentrating hormone system of the rat brain: an immuno- and hybridization histochemical characterization, J. Comp. Neurol. 319 (2) (1992) 218-245.

[6] S. Knollema, et al., Novel hypothalamic and preoptic sites of prepro-melaninconcentrating hormone messenger ribonucleic acid and peptide expression in lactating rats, J. Neuroendocrinol. 4 (6) (1992) 709-717.

[7] T.A. Rondini, et al., Chemical identity and connections of medial preoptic area neurons expressing melanin-concentrating hormone during lactation, J. Chem. Neuroanat. 39 (1) (2010) 51-62.

[8] J.C. Bittencourt, Anatomical organization of the melanin-concentrating hormone peptide family in the mammalian brain, Gen. Comp. Endocrinol. 172 (2) (2011) $185-197$.

[9] L.B. Cook, L. Shum, S. Portwood, Melanin-concentrating hormone facilitates migration of preadipocytes, Mol. Cell. Endocrinol. 320 (1-2) (2010) 45-50.

[10] M.C. Beinfeld, et al., The brain decade in debate: VIII. Peptide hormones and behavior: cholecystokinin and prolactin, Braz. J. Med. Biol. Res. 34 (11) (2001) 1369-1377.

[11] D. Qu, et al., A role for melanin-concentrating hormone in the central regulation of feeding behaviour, Nature 380 (6571) (1996) 243-247.

[12] M.I. Gonzalez, B.I. Baker, C.A. Wilson, Stimulatory effect of melanin-concentrating hormone on luteinising hormone release, Neuroendocrinology 66 (4) (1997) 254-262.

[13] M.I. Gonzalez, et al., Alpha-melanocyte-stimulating hormone (alpha-MSH) and melanin-concentrating hormone ( $\mathrm{MCH})$ modify monoaminergic levels in the preoptic area of the rat, Peptides 18 (3) (1997) 387-392.

[14] L. Benedetto, et al., Melanin-concentrating hormone in the medial preoptic area reduces active components of maternal behavior in rats, Peptides 58 (2014) 20-25.

[15] C. Kenyon, P. Cronin, P. Malinek, Effects of lidocaine on nipple attachment and home orientation by rat pups, Behav. Neural Biol. 32 (2) (1981) 261-264.

[16] J. Lonstein, et al., Forebrain expression of c-fos due to active maternal behaviour in lactating rats, Neuroscience 82 (1) (1997) 267-281.

[17] I.C. Cespedes, et al., mRNA expression of corticotropin-releasing factor and urocortin 1 after restraint and foot shock together with alprazolam administration, Peptides 31 (12) (2010) 2200-2208.

[18] A.S. Fleming, et al., Activation of Fos-like immunoreactivity in the medial preoptic area and limbic structures of maternal and social interactions in rats, Behav. Neurosci. 108 (4) (1994) 724.
[19] M. Numan, M.J. Numan, Importance of pup-related sensory inputs and maternal performance for the expression of Fos-like immunoreactivity in the preoptic area and ventral bed nucleus of the stria terminalis of postpartum rats, Behav. Neurosci. 109 (1) (1995) 135.

[20] T.P. Sheehan, et al., Using c-Fos immunocytochemistry to identify forebrain regions that may inhibit maternal behavior in rats, Behav. Neurosci. 114 (2) (2000) 337.

[21] L.J. Grota, R. Ader, Continuous recording of maternal behaviour in Rattus norvegicus, Anim. Behav. 17 (4) (1969) 722-729.

[22] C.P. Cramer, E. Thiels, J.R. Alberts, Weaning in rats: I. Maternal behavior, Dev. Psychobiol. 23 (6) (1990) 479-493.

[23] E. Thiels, J.R. Alberts, C.P. Cramer, Weaning in rats: II. Pup behavior patterns, Dev. Psychobiol. 23 (6) (1990) 495-510.

[24] K. Ota, A. Yokoyama, Body weight and food consumption of lactating rats: effects of ovariectomy and of arrest and resumption of suckling, J. Endocrinol. 38 (3) (1967) 251-261.

[25] A.P. Coll, I.S. Farooqi, S. O'Rahilly, The hormonal control of food intake, Cell 129 (2) (2007) 251-262.

[26] G.J. Morton, et al., Central nervous system control of food intake and body weight, Nature 443 (7109) (2006) 289-295.

[27] M.W. Schwartz, et al., Central nervous system control of food intake, Nature 404 (6778) (2000) 661-671.

[28] L.V. Sita, C.F. Elias, J.C. Bittencourt, Connectivity pattern suggests that incertohypothalamic area belongs to the medial hypothalamic system, Neuroscience 148 (4) (2007) 949-969.

[29] S. Hansen, A. Ferreira, Food intake, aggression, and fear behavior in the mother rat: control by neural systems concerned with milk ejection and maternal behavior, Behav. Neurosci. 100 (1) (1986) 64.

[30] A.H. McIVER, W.E. Jeffrey, Strain differences in maternal behavior in rats, Behaviour 28 (1) (1967) 210-216.

[31] M. Pereira, J.I. Morrell, The changing role of the medial preoptic area in the regulation of maternal behavior across the postpartum period: facilitation followed by inhibition, Behav. Brain Res. 205 (1) (2009) 238-248.

[32] A. Viale, et al., The melanin-concentrating hormone gene in human: flanking region analysis, fine chromosome mapping, and tissue-specific expression, Mol. Brain Res. 46 (1) (1997) 243-255.

[33] J.S. Lonstein, et al., Maternal behavior stimulates c-fos activity within estrogen receptor alpha-containing neurons in lactating rats, Neuroendocrinology 72 (2) (2000) 91-101.

[34] P.J. Shughrue, M.V. Lane, I. Merchenthaler, Comparative distribution of estrogen receptor- $\alpha$ and- $\beta$ mRNA in the rat central nervous system, J. Comp. Neurol. 388 (4) (1997) 507-525.

[35] J.W. Muschamp, E.M. Hull, Melanin concentrating hormone and estrogen receptor$\alpha$ are coextensive but not coexpressed in cells of male rat hypothalamus, Neurosci. Lett. 427 (3) (2007) 123-126.

[36] J.P. Kukkonen, Physiology of the orexinergic/hypocretinergic system: a revisit in 2012, Am. J. Physiol. Cell Physiol. 304 (1) (2013) C2-32. 


\section{Chapter 6}

\section{The weaning period promotes alterations in the orexin neuronal population of rats in a suckling- dependent manner}

Diniz GB, Candido PL, Klein MO, Alvisi RD, Presse F, Nahon J-L, Felicio LF and Bittencourt JC

As published in: Brain Structure and Function [ePub ahead of print]. 2018. DOI: $10.1007 / s 00429-018-1723-0$ 



\title{
The weaning period promotes alterations in the orexin neuronal population of rats in a suckling-dependent manner
}

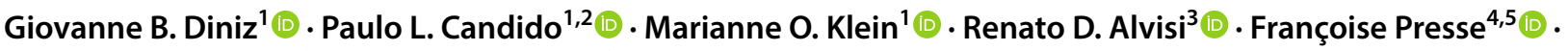 \\ Jean-Louis Nahon ${ }^{4,5,6}\left[\right.$ - Luciano F. Felicio ${ }^{3}\left[\right.$. Jackson C. Bittencourt ${ }^{1,7} \mathbb{C}$
}

Received: 29 August 2017 / Accepted: 7 July 2018

○) Springer-Verlag GmbH Germany, part of Springer Nature 2018

\begin{abstract}
The orexin-immunoreactive neurons are part of an important arousal-promoting hypothalamic population. Several groups have investigated these neurons during the lactation period, when numerous physiological alterations occur in the dam's body to cope with the newly acquired metabolic needs of the litter. Although those studies have probed this population during the early and intermediate stages of lactation, few works have examined its response to weaning, including the cessation of the tactile suckling stimulus as the litter stops nursing. Using double immunohistochemistry for orexin and FOS combined with three-dimensional reconstruction techniques, we investigated orexin-synthesizing neurons and their activation at different times during weaning, in addition to the role played by the suckling stimulus. We report here that weaning promoted a decline in the anterior population of orexin-immunoreactive neurons and decreased the number of double orexin-FOS neurons labeled in the central dorsomedial hypothalamus, in addition to reducing the overall number of FOS-immunoreactive cells in the whole tuberal hypothalamus. Disruption of the suckling stimulus from the pups impaired the decrease in the number of anteriorly located orexin-immunoreactive neurons, attenuated the activation of orexin-synthesizing cells in the dorsomedial hypothalamus and reduced the number of FOS-immunoreactive neurons across the tuberal hypothalamus. When taken together, our data suggest that the weaning period is necessary to restore neurochemical pathways altered during the lactation period and that the suckling stimulus plays a significant role in this process.
\end{abstract}

Keywords Hypocretin $\cdot$ Hypothalamus $\cdot$ Neuropeptides $\cdot$ Sleep $\cdot$ Wakefulness $\cdot$ Lactation

Electronic supplementary material The online version of this article (https://doi.org/10.1007/s00429-018-1723-0) contains supplementary material, which is available to authorized users.

Jackson C. Bittencourt

jcbitten@icb.usp.br

1 Laboratory of Chemical Neuroanatomy, Department of Anatomy, Institute of Biomedical Sciences, University of São Paulo, São Paulo 05508-000, Brazil

2 Department of Anatomy, Santa Marcelina Medical School, São Paulo, Brazil

3 Department of Pathology, School of Veterinary Medicine, University of São Paulo, São Paulo, Brazil

4 CNRS/UNS UMR 7275, Institut de Pharmacologie Moléculaire et Cellulaire, Sophia Antipolis, Valbonne, France

5 Université Côte d'Azur, Nice, France

6 Station de Primatologie UPS846 CNRS, Rousset-sur-Arc, France

7 Center for Neuroscience and Behavior, Institute of Psychology, University of São Paulo, São Paulo, Brazil

\section{Introduction}

The orexinergic peptidergic system consists of two peptides: orexin A (ORXA), also known as hypocretin-1 (HRC-1), and orexin B (ORXB), also known as hypocretin-2 (HRC-2). These two peptides originate from the same preprohormone, prepro-orexin (ppORX), encoded by the Hcrt gene. The sequences of ORXA and ORXB are remarkably conserved in all mammals studied: ORXA is a cyclic peptide with 32 amino acids, while ORXB is a linear peptide with 28 amino acids (de Lecea et al. 1998; Sakurai et al. 1998; Tsujino and Sakurai 2009). Orexin-immunoreactive (ORX-ir) neurons in the rat are found exclusively in the tuberal hypothalamus, predominantly occupying the lateral hypothalamic area (LHA), with a high concentration of immunoreactive cells present in the perifornical nucleus (de Lecea et al. 1998; Sakurai et al. 1998). Fibers immunoreactive to ORX are found in several subcortical areas of the central nervous system (CNS) but are seldom found in cortical fields (Date et al. 
1999; Nambu et al. 1999; Peyron et al. 1998). As there is no difference in staining pattern between an antibody selective for ORXA and one that stains for both ORXA and ORXB, it is suggested that there is a high degree of colocalization between the two peptides (Peyron et al. 1998).

The ORX system has been implicated in several essential physiological processes, such as regulation of the sleep/wake architecture (Hagan et al. 1999; Chemelli et al. 1999; Branch et al. 2016); arousal, attention and behavioral responses to stress (Furlong et al. 2009; Mahler et al. 2014); reward to cue-associated stimuli (Mahler and Aston-Jones 2012; Smith and Aston-Jones 2012); hedonic enhancement of "liking" reactions and feeding (Ho and Berridge 2013) and locomotor activity and energy expenditure (Wang et al. 2001; Hagan et al. 1999). In many cases, however, orexin function is complex and changes according to the experimental paradigm and the context of the animal. Despite the initial description of its orexigenic properties (Sakurai et al. 1998; Volkoff et al. 1999; Haynes et al. 1999; Edwards et al. 1999), it has been recently suggested that the net effect of ORX action is weight-loss due to increased energy expenditure, and ORX neurons may even oppose eating, with decreased activity of ORX-synthesizing neurons upon eating onset (González et al. 2016). A possible unifying role of orexin is as motivational activator, increasing the overall animal motivation to respond to certain environmental cues depending on its metabolic state (Mahler et al. 2014).

The broad range of physiological and behavioral actions of ORX has drawn the attention of several groups to the participation of this peptidergic system in the physiological changes that occur during the lactation period. This period is characterized by a substantial increase in food consumption by the dam, whose diet must provide nutrition for her and her entire litter (Fleming 1976; Ota and Yokoyama 1967). There is no consensus, however, as to what role ORX plays in lactation or what stimuli may modulate this system (Brogan et al. 2000; Cai et al. 2001; Garcia et al. 2003; Sun et al. 2003; Wang et al. 2003; España et al. 2004). Although several studies have examined the initial and intermediate stages of lactation, the weaning period has often been overlooked in the literature. In laboratory animals, the weaning period starts around the 15th day of lactation (after the eruption of the incisor teeth in the pups at the 14th day) and is marked by a switch in the litter from an entirely milk-based, damdependent diet to an autonomous solid diet (Thiels et al. 1990; Cramer et al. 1990). In the 22nd day postpartum, the pups are separated from the dam, and lactation is terminated as the mother resumes sexual behavior. The weaning period encompasses some of the greatest changes in the physiology of the dam, including a steep increase in food consumption (Ota and Yokoyama 1967). Considering the available data, we hypothesized that ORX synthesis and the activity of ORX-ir neurons might change during the weaning period, resulting in increased food consumption. We also investigated the possibility that the suckling stimulus from the pups is necessary for the modulation of these ORX-ir neurons.

\section{Materials and methods}

\section{Animals}

Adult female Wistar rats $(n=75)$ at approximately 90 days of age were employed in this study. All rats were bred and raised in the animal facility of the Department of Pathology, School of Veterinary Medicine, University of São Paulo. The animals were individually housed in polypropylene cages $(30 \mathrm{~cm} \times 40 \mathrm{~cm} \times 18 \mathrm{~cm})$ with approximately $1 \mathrm{~L}$ of medium-grade pine flakes as bedding, under a $12 \mathrm{~h}: 12 \mathrm{~h}$ light cycle (lights on at 6:00 AM) and controlled temperature $\left(23 \pm 2{ }^{\circ} \mathrm{C}\right)$. All animals had access to chow and water ad libitum throughout the experiment. The procedures were performed following the National Institutes of Health Guide for the Care and Use of Laboratory Animals (2010) and were also evaluated and approved by the Committee on Care and Use of Animals Resources, School of Veterinary Medicine, University of São Paulo (Process \#2185/2011).

\section{Mating}

All female rats had their estrous cycles tracked by the method of vaginal cytology (Marcondes et al. 2002). After confirmation of two regular consecutive estrous cycles, females were randomly separated in two groups. The first group ( $n=10$; baseline, BL) was euthanized in the afternoon of the diestrus or proestrus day ( $n=5$ each). Animals in the second group $(n=65)$ were placed in the cage of sexually experienced males on the afternoon of the following proestrus. The next day, the presence of sperm in the vaginal cytology sample was considered indicative of mating, and the day was designated day 1 of pregnancy. On postpartum day (PPD) 2, each litter was culled to eight pups (four males and four females).

\section{Suckling interruption}

During the progression of lactation, the mothers and their litters were habituated to handling by the experimenter but otherwise were left undisturbed. On PPD14, dams were randomly assigned to three groups: with a suckling stimulus (WS; $n=28$ ), without a suckling stimulus (NS; $n=28$ ), and pup removal (PR; $n=9$ ). The vibrissal pad anesthesia technique was performed to block the suckling stimulus (Alvisi et al. 2016; Kenyon et al. 1981; Lonstein et al. 1997). Briefly, the pups were separated from the mother one hour before the test and received a topical cream of $5 \%$ lidocaine (Xylocaine 
$50 \mathrm{mg} / \mathrm{g}$ - AstraZeneca; London, UK) on their vibrissal pads, resulting in complete loss of vibrissal reflexes. In the WS group, the pups were separated from the mother but did not receive the anesthetic cream. After one hour, the pups were returned to the mother, and their capability to attach to the nipples was visually assessed. In cases, where pups in the NS group were able to attach to the nipples, the mothers were excluded from the experiment. Neither group showed any impairment in the ability of pups to vocalize, and there was no difference between groups in maternal pup retrieval, grooming or nest building. After 20 min undisturbed, the pups were once more separated from the mother. This experiment was performed on PPD15, PPD17, PPD19 and PPD21, following the early and middle stages of weaning before the standard laboratory practice of separating the pups on PPD22 (Thiels et al. 1990). In the PR group, composed of females on PPD15 $(n=5)$ and PPD21 $(n=4)$, pups were removed on the first separation but were not returned to the dam at any point. The dams were perfused ninety minutes after separation.

\section{Immunohistochemistry}

Before the sacrifice, dams were anesthetized with a lethal intraperitoneal dose of $2 \%$ xylazine $(5 \mathrm{mg} / \mathrm{kg}$ ) and $5 \%$ ketamine $(30 \mathrm{mg} / \mathrm{kg})$. The dams were then intracardially perfused with a cold $0.9 \%$ saline solution $(150 \mathrm{ml}-5 \mathrm{~min})$, followed by $750 \mathrm{ml}$ of cold $4 \%$ formaldehyde in phosphate buffer ( $\mathrm{pH} 7.4 ; 30 \mathrm{ml} / \mathrm{min}$ ). Once the perfusion was complete, the brains were harvested and postfixed overnight in the same fixative supplemented with $20 \%$ sucrose. The collected samples were then transferred to a $20 \%$ sucrose solution in $0.02 \mathrm{M}$ potassium-sodium phosphate-buffered saline (KPBS) until sectioning. The brains were frozen in dry ice, and series of $30 \mu \mathrm{m}$ frontal sections (1:5 series) were obtained with a freezing microtome. A random number from 1 to 75 was associated with each brain for identification.

For double ORX and FOS labeling, we employed the immunohistochemistry method described by Hoffman et al. (2008). Briefly, one free-floating series of brain sections from each animal was washed in KPBS and had its endogenous peroxidase activity quenched by incubation with $0.3 \%$ hydrogen peroxide in KPBS (Sigma Chemical; St. Louis, MO, USA) for $15 \mathrm{~min}$, followed by $6 \times 5 \mathrm{~min}$ rinses in KPBS. The slices were then incubated with anti-FOS rabbit polyclonal primary antibody (Table 1) in 3\% normal goat serum and $0.3 \%$ Triton X-100 diluted in KPBS for $24 \mathrm{~h}$ at room temperature. The sections were subsequently rinsed in KPBS and incubated for one hour at room temperature with biotinylated goat anti-rabbit IgG (1:800 Vector \#S-1000Vector Laboratories; Burlingame, CA, USA) in KPBS and $0.3 \%$ Triton X-100. This step was followed by KPBS rinses and incubation for $1 \mathrm{~h}$ with an avidin-biotin-HRP solution (1:333 Vectastain Elite \#PK-6100; Vector) in KPBS. For black chromogen deposition, the slices were incubated with 3,3'-diaminobenzidine tetrahydrochloride (DAB, $0.02 \%$; Sigma), $0.003 \%$ hydrogen peroxide and $2.5 \%$ nickel ammonium sulfate (NAS) diluted in $0.2 \mathrm{M}$ sodium acetate buffer (pH 6.5). After $20 \mathrm{~min}$, the reaction was interrupted by successive washes in acetate buffer and KPBS. For ORX labeling, the reaction described was repeated in the same slices by applying an anti-ORXA rabbit polyclonal primary antibody (Table 1) and performing chromogen deposition without NAS for $20 \mathrm{~min}$ to obtain a light brown coloring. The

Table 1 Primary antibodies employed in this work

\begin{tabular}{|c|c|c|c|c|c|c|c|}
\hline $\begin{array}{l}\text { Antibody common } \\
\text { name }\end{array}$ & Manufacturer & Man code & References & PubMed ID & $\begin{array}{l}\text { NIF antibody } \\
\text { registry }\end{array}$ & Antigen sequence & $\begin{array}{l}\text { Application/ } \\
\text { concentration }\end{array}$ \\
\hline $\begin{array}{l}\text { Rabbit anti orexin } \\
\text { A [anti-ORX] }\end{array}$ & $\begin{array}{l}\text { Phoenix Pharma- } \\
\text { ceuticals, Inc. } \\
\text { [Burlingame, } \\
\text { CA, USA] }\end{array}$ & $\mathrm{H}-003-30$ & Chen et al. (1999) & 10076892 & AB_2315019 & Whole protein & 1:2'000 [IP] \\
\hline $\begin{array}{l}\text { Rabbit anti } \\
\text { [human] FOS } \\
\text { [anti-FOS] }\end{array}$ & $\begin{array}{l}\text { Merck [former } \\
\text { Oncogene] } \\
\text { [Darmstadt, } \\
\text { Germany] }\end{array}$ & PC38 & Tseng et al. (1997) & 9090643 & AB_2106755 & $\begin{array}{l}\text { SGFNADYEAS- } \\
\text { SSRC }\end{array}$ & $\begin{array}{l}1: 70^{\prime} 000[\mathrm{IP}] \\
1: 35^{\prime} 000[\mathrm{BT}]\end{array}$ \\
\hline $\begin{array}{c}\text { Mouse anti-NeuN } \\
\text { (clone A60) } \\
\text { [anti-NeuN] }\end{array}$ & $\begin{array}{l}\text { Merck [former } \\
\text { Calbiochem] } \\
\text { [Darmstadt, } \\
\text { Germany] }\end{array}$ & MAB377 & $\begin{array}{l}\text { Sarnat et al. } \\
\text { (1998) }\end{array}$ & 9545178 & AB_2298771 & Whole protein & $1: 2^{\prime} 000[\mathrm{IF}]$ \\
\hline $\begin{array}{l}\text { Mouse anti glial } \\
\text { fibrillary acidic } \\
\text { protein (clone } \\
\text { G-A-5) [anti- } \\
\text { GFAP] }\end{array}$ & $\begin{array}{l}\text { Sigma-Aldrich } \\
\text { [St. Louis, MO, } \\
\text { USA] }\end{array}$ & G3893 & $\begin{array}{l}\text { Burnashev et al. } \\
\text { (1992) }\end{array}$ & 1317970 & AB_477010 & Whole protein & $1: 2^{\prime} 000[\mathrm{IF}]$ \\
\hline
\end{tabular}

$B T$ biotinylated tyramine fluorescence immunohistochemistry, $I F$ immunofluorescence, $I P$ immunoperoxidase 
sections were then mounted on gelatin-coated glass slides in rostrocaudal order, dehydrated, defatted and coverslipped with a hydrophobic mounting medium (DPX; Sigma).

Double immunofluorescence was performed to verify whether the activated FOS-ir cells were neuronal or glial. Briefly, sections were rinsed in KPBS and incubated with $0.3 \%$ hydrogen peroxide in KPBS (Sigma Chemical) for $15 \mathrm{~min}$, followed by a new round of rinses in KPBS. The slices were then incubated with anti-FOS (Table 1) in 3\% normal goat serum and $0.3 \%$ Triton $\mathrm{X}-100$ for $24 \mathrm{~h}$ at room temperature. The sections were then rinsed in KPBS and incubated for one hour with biotinylated goat anti-rabbit IgG (1:5000; Vector), followed by $30 \mathrm{~min}$ of incubation with avidin-biotin-HRP solution (1:833; Vector) in KPBS and $20 \mathrm{~min}$ in $0.5 \%$ biotinylated (EZ-Link sulfo-NHS-LC-biotin; ThermoFisher Scientific, Waltham, MA, USA) tyramine (Sigma-Aldrich) and hydrogen peroxide $(0.005 \%)$. After new washes in KPBS, the slices were incubated with Alexa Fluor 488-conjugated streptavidin (1:200-Molecular Probes; Eugene, OR, USA) for $2 \mathrm{~h}$ at room temperature, rinsed and incubated with anti-NeuN or anti-GFAP primary antibodies (Table 1) at room temperature, as described before. The next day, the slices were washed in KPBS and incubated with Alexa Fluor 594-conjugated goat anti-mouse $\operatorname{IgG}(\mathrm{H}+\mathrm{L})$ (1:200-molecular probes), washed, mounted on glass slides, dried at room temperature and coverslipped with ProLong Diamond antifade mountant (ThermoFisher).

\section{Object counting}

The distribution of stained cells was examined in the tuberal hypothalamus of all animals and three-dimensionally reconstructed. Toward that end, we used the Neurolucida 11.03 software package (MBF Bioscience Co.; Williston, VT, USA) operating a computer-driven motorized stage (Ludl Electronic Products; Hawthorne, NY, USA) of an Eclipse 80i microscope (Nikon Instruments; Tokyo, Japan) equipped for brightfield and darkfield imaging. Nine slices from each animal were reconstructed, encompassing the tuberal hypothalamus as described by Saper et al. (1979) and roughly corresponding to -2.30 to $-3.30 \mathrm{~mm}$ from bregma (Paxinos and Watson 2013).

The tuberal hypothalamus of each animal was further subdivided into three sectors: the first three slices were designated the anterior sector, the three following slices were designated the central sector, and the final three slices were designated the posterior sector (Fig. 1). The location of the fornix was determined using a low-power magnifying lens with a darkfield condenser, and a four-quadrant probe was placed over it (Fig. 1). With brightfield illumination and $20 \times$ or $40 \times$ objectives, three different markers were then placed over the stained cells: cyan markers for ORXA-ir cells, magenta markers for FOS-ir cells and yellow markers for double ORXA/FOS-ir cells. This process was repeated for all slices, and the total number of markers in each quadrant was extracted from the samples through Neurolucida Explorer 11.03 software (MBF Bioscience). The experimenter responsible for object counting was informed only of the identification numbers of the animals and was completely blind to the experimental parameters of the samples.

\section{Imaging}

Brightfield photomicrographs were obtained with a Nikon Eclipse 80i microscope (Nikon) coupled to a digital camera (CX3000-MBF Bioscience) through Microlucida 3.03 acquisition software (MBF Bioscience). Immunofluorescence photomicrographs were obtained with a Leica DMR (Leica Microsystems Inc; Wetzlar, Germany) coupled to a DS-Ri1 camera (Nikon) through NIS-Elements BR 3.2 acquisition software (Nikon). The different fluorophores were acquired in an emulated monochromatic mode in separate channels and then merged. All images had adjustments in their brightness, contrast, and sharpness applied to every pixel in the picture, and the changes did not alter the information illustrated in the figures. The plates were assembled, and off-tissue background/ methodological artifacts were cleared by means of Adobe Photoshop CC 2017.0.1 software (Adobe Systems Inc; San Jose, CA, USA).

\section{Statistical analyses}

For all statistical analyses, the individual values from each animal were employed. Once every animal was grouped according to its experimental condition (day of lactation and suckling stimulus), outliers were identified by Grubbs' test and removed from the analysis. The measurements that were not excluded from this work were then normalized by a $\log _{10}$ transformation. Differences between group means were analyzed by two-way ANOVA with day of lactation (15-21) and suckling (WS/NS) as factors and were then corrected for multiple comparisons by Tukey's test. In specific cases, samples were individually analyzed by one-way ANOVA (with lactation or estrous cycle day as the factor) with Tukey's range test or an unpaired $t$ test with Welch's correction (suckling effect). In all cases, a $p<0.05$ was defined as statistically significant. The graphs are based on the mean \pm standard error of the mean (SEM). All calculations were performed using the statistical analysis software Prism 6 (GraphPad; San Diego, CA, USA). 

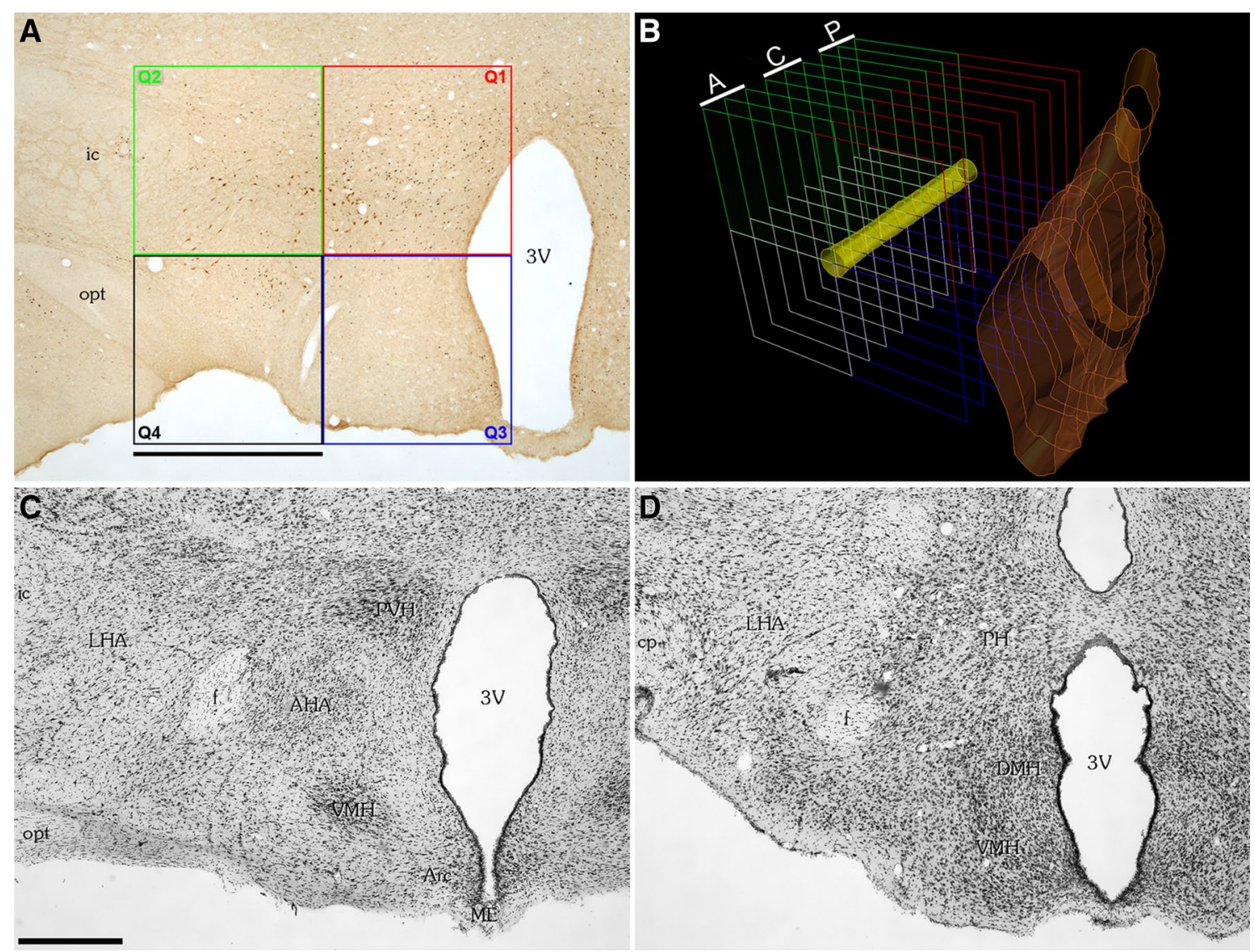

Fig. 1 Employed probe and object counting method. a Four-quadrant probe was used for each of the nine frontal slices analyzed per animal. Each quadrant had a total area of $960,400 \mu \mathrm{m}^{2}$. The common vertex among all four quadrants was placed in the middle of the fornix for each slice to ensure reproducibility between slices and animals. b Isometric view of the nine consecutive probes employed in each animal. The three first probes were counted as the anterior sector (A); the following three probes were counted as the central sector (C); and the final three probes were counted as the posterior sector $(\mathrm{P})$.

\section{Results}

\section{Orexin-immunoreactive neurons}

The majority of ORX-ir neurons were found in quadrant (Q) 1 and Q2 in all sectors, with the population in these two quadrants representing $85 \pm 5.9 \%$ (mean $\pm \mathrm{SD}$ ) of the total ORX population (Fig. 2). For this reason, the analysis of ORX-ir neurons will focus on these two quadrants. The three-dimensional reconstruction is available to the reader in VRML format in Online Resource 1.

In anterior Q1, there was a significant interactive effect of suckling and day of lactation on the number of ORX-ir neurons $[F(3,16)=7.076 ; p=0.0031$-Fig. $3 a)$. In the WS group, there was a decrease in the number of ORX-ir neurons as the weaning period progressed (PPD15 vs. PPD21: $p<0.01$; PPD17 vs. PPD21: $p<0.05$ ), while there was no
The relative positions of the fornix (yellow) and of the third ventricle (orange) are indicated in the figure. $\mathbf{c}$ Brightfield photomicrograph of a frontal slice subjected to Nissl staining, illustrating the main cytoarchitectonic landmarks of the first slice reconstructed from each animal. d Brightfield photomicrograph of a frontal slice subjected to Nissl staining, illustrating the main cytoarchitectonic landmarks of the last slice reconstructed from each animal. Scale bar: a $980 \mu \mathrm{m}$; c, d $100 \mu \mathrm{m}$

clear pattern to the variation in the number of ORX-ir neurons in the NS group. This difference ultimately resulted in the stimulated PPD21 animals displaying significantly fewer ORX-ir neurons than the non-stimulated group (PPD21WS vs. PPD21NS: $p<0.05$-Fig. 3a). This lead us to hypothesize that ORX-ir neurons return during weaning to a baseline number of neurons. To test that hypothesis, we investigated the number of ORX neurons of rats in diestrus and proestrus without any stimulus. The number of ORX-ir neurons in PPD15 was significantly higher than both the Diestrus and Proestrus groups (PPD15 WS vs. Diestrus: $p<0.01$; PPD15 WS vs. Proestrus: $p<0.001$; PPD15 NS vs. Proestrus: $p<0.05-$ Online Resource 2). When the PPD21 groups were compared to the baseline groups, there was no difference in the PPD21 WS group, but a statistically significant difference between PPD21 NS and Proestrus $(p<0.05)$. This was the only sector, where lactation values 


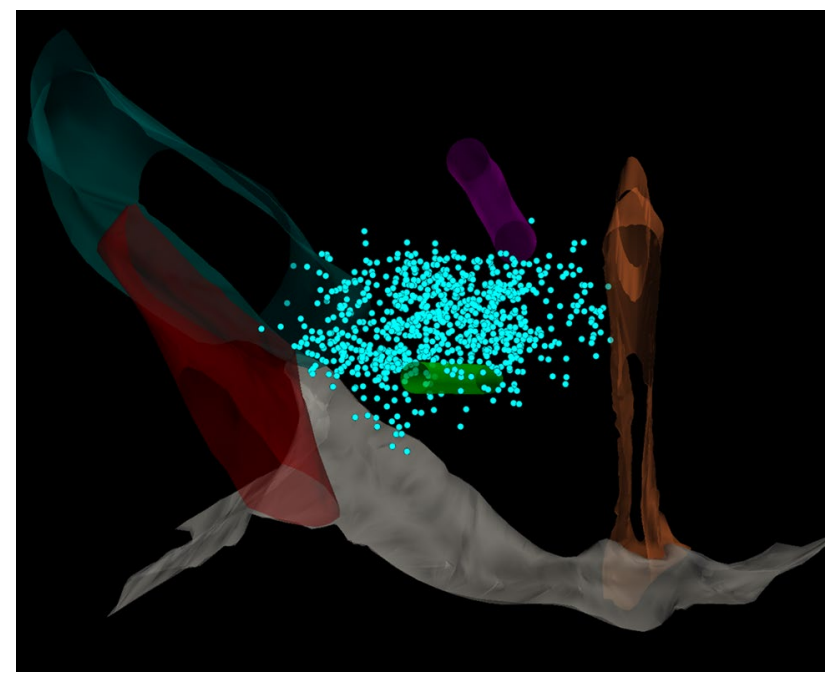

Fig. 2 Distribution of orexin-immunoreactive neurons in the tuberal hypothalamus of a rat dam. Three-dimensional reconstruction based on nine semi-serial frontal slices of the brain of a rat dam at the 15 th day of lactation, showcasing the distribution of orexin-immunoreactive neurons in the tuberal hypothalamus. Each cyan dot represents a single orexin-immunoreactive cell. The outlined structures are as follows: green-fornix; purple-mammillothalamic tract; orange-third ventricle; dark green-internal capsule/cerebral peduncle; red-optic tract; gray—ventral floor

statistically differed from baseline values. When compared to each other, there was no difference between Diestrus and Proestrus $(p=0.1766)$.

In anterior Q2, there was also a significant interactive effect of suckling stimulus and lactation day on the mean number of ORX-ir neurons present in this area $[F(3,16)=5.409 ; p=0.0092-F i g$. 3a'), with lactation day displaying an increased participation in the difference between means $(F(3,16)=0.0273)$. There was a higher number of ORX-ir neurons on PPD21 in the NS group than in either group on PPD19 (PPD19WS vs. PPD21NS: $p<0.05$; PPD19NS vs. PPD21NS: $p<0.05)$, while this increase was not observed in the WS group (Fig. 03a'). The decrease in ORX-ir cells in the anterior sector between PPD15 and PPD21 is illustrated in Fig. 4.

In contrast to the anterior sector, there was no evidence of variation in the number of ORX-ir neurons in the central Q1 (Fig. 03b). On the other hand, there was an alteration in the number of ORX-ir neurons in central Q2, with a strong interaction between suckling and day of lactation $(F(3,16)=10.9$; $p=0.004)$, and the lactation day was the predominant factor $\left(F(3,16)=3.257 ; p=0.0492-F i g .3 b^{\prime}\right)$. Upon further investigation, there was a strong effect of lactation day in the WS group $(F(3,8)=11.54 ; p=0.0028)$, with an increase from PPD15 to PPD19 and PPD21. This was not the case in the NS group, as the day of lactation did not affect the number of ORX-ir neurons $(F(3,8)=2.785 ; p=0.1097-$ Fig. 3b').
In the posterior division, the number of Q1 ORXir neurons displayed suckling-dependent variation $(F(1,16)=6.753 ; p=0.0194-F i g .3 c)$. Upon pairwise comparison, however, there was no effect of suckling stimulus on the number of ORX-ir neurons for each day of lactation, suggesting that the variation has a small magnitude or simply reflects noise in the sample. There was no statistically significant alteration in the number of ORX-ir neurons in the posterior Q2 (Fig. 3c').

Since ORX has been associated to stress responses (Furlong et al. 2009), and the abnormal behavior of the pups unable to suckle on the mother could have a stressor effect on the dam, we generated two groups with complete litter removal from the female on the day of testing (PPD15 PR and PPD21 PR). Except for the pup return, all other dam manipulations were performed as described. There was no statistical difference between PPD15 NS and PPD15 PR ( $p=0.49$-Online Resource 2). Regarding PPD21, the number of neurons in the pup removal group was statistically higher than the number of groups in the stimulated group (PPD21 WS vs. PPD21 PR: $p<0.05-O n l i n e$ Resource 2), but there was just a non-significant decrease in PPD21 PR when compared to PPD21 NS ( $p=0.224)$. The fact that the values of the NS groups were slightly higher than values on the PR groups suggest stress or other modalities of pup stimuli play a role in the observed results. However, the small magnitude of this effect (as revealed by the lack of statistical significance upon pairwise comparison) suggest that those effects cannot explain, alone, the results observed in this work.

\section{FOS-immunoreactive cells}

A high number of FOS-ir cells was detected in the tuberal hypothalamus of dams, with a greater staining density in the periventricular and medial zones of the hypothalamus and a lesser density in the lateral zone (Fig. 5-the three-dimensional reconstruction is available in VRML format in Online Resource 3). Double NeuN and FOS staining revealed that approximately $96 \%$ of FOS-labeled cells were immunoreactive to NeuN, but there was no colocalization between FOS and GFAP, suggesting that complete overlap of markers was not achieved as a result of faint or incomplete staining for NeuN (Online Resource 4). These results indicate that hypothalamic FOS activation during the lactation period occurs predominantly in neuronal cells.

Regarding the number of FOS-ir neurons in the anterior Q1, there was a significant effect of lactation day on this parameter $(F(3,16)=9.380 ; p=0.0008)$, with an overall decrease in the number of FOS-ir neurons in the anterior Q1 as lactation progressed (Fig. 6a). Upon further investigation, however, there was a difference between the WS and NS groups, with animals in the WS group displaying only 
Fig. 3 Variations in the number of orexin-immunoreactive neurons for different sectors, quadrants, days of lactation and stimulation conditions. Each bar represents the mean \pm SEM for each day of lactation and stimulation condition. $* p<0.05$; $* * p<0.01$
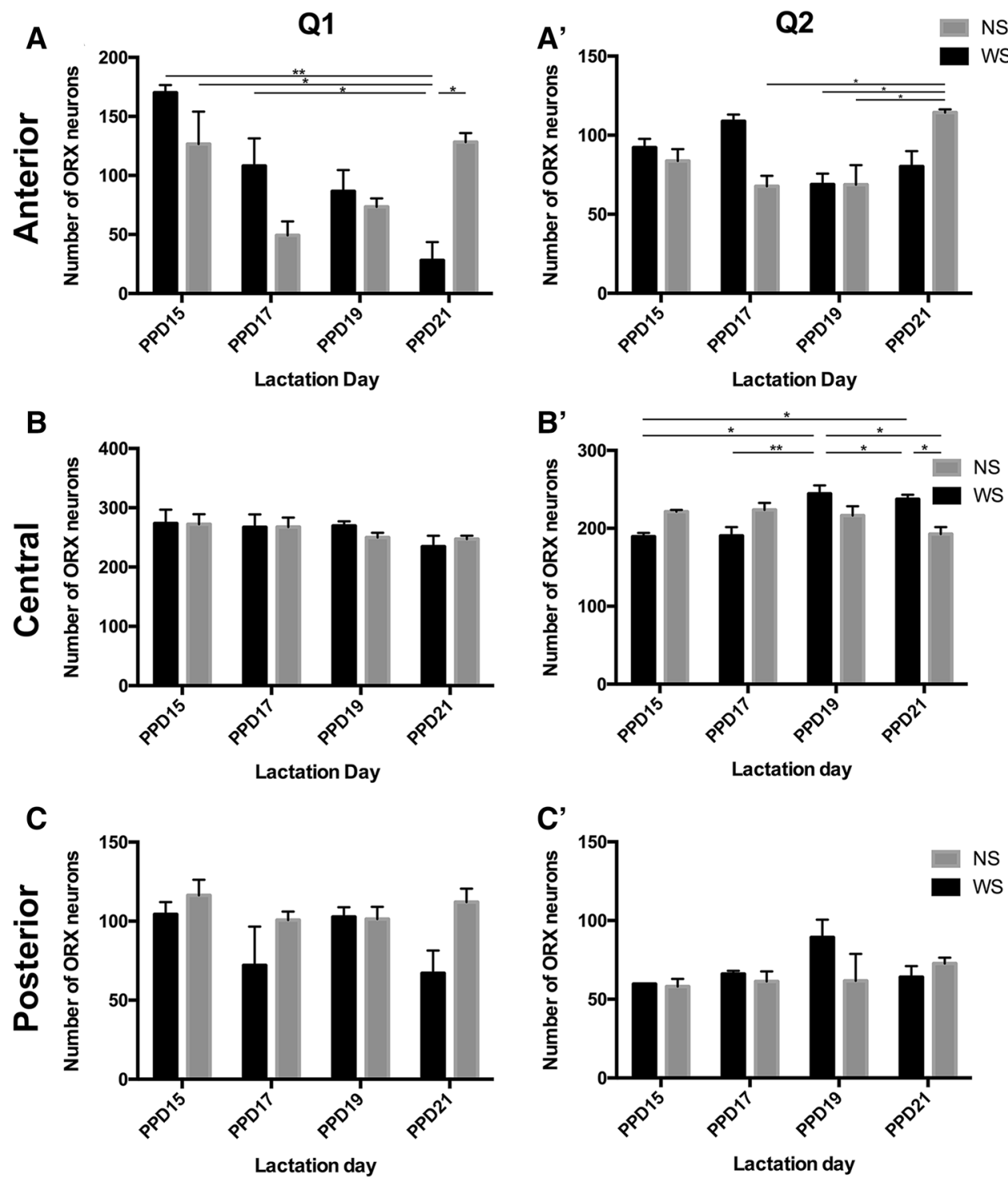

a decreasing trend $(F(3,8)=3.544 ; p=0.0677)$, while the NS group reached statistical significance $(F(3,8)=9.288$; $p=0.0055$-Fig. 6a). Regarding the number of FOS-ir neurons in anterior Q2, only the lactation day appears to influence the number of FOS-ir neurons $(F(3,16)=4.847$; $p=0.0138$-Fig. 6b). When the groups were investigated separately, there was no effect of lactation day in the WS group $(F(3,8)=1.454 ; p=0.2981)$, while there was a trend in the NS group $(F(3,8)=3.777 ; p=0.0590-$ Fig. $6 b)$. This difference suggests that the suckling effect may serve to slow down a progressive decrease in the activation of LHA neurons in the late lactation period. In anterior Q3, suckling played a discrete but statistically significant role in the number of FOS-ir neurons $(F(1,16)=4.710$; $p=0.0454$-Fig. 6c). Dams in the WS group had an overall higher number of FOS-ir neurons, and this effect became statistically significant on PPD21 (Fig. 6c). In anterior
Q4, both suckling stimulus and lactation day were statistically significant factors in the number of FOS-ir neurons (suckling $-F(1,16)=26.64 ; p<0.0001$ I day of lactation$F(3,16)=5.427, p=0.0091)$, but there was only a trend of interaction between these two factors $(F(3,16)=3.013$, $p=0.0608$ ), with an overall higher number of FOS-ir neurons in the stimulated group than in the NS group (Fig. 6d).

The number of FOS-ir neurons in central Q1 was strongly affected by lactation day $(F(3,16)=15.39$; $p<0.0001)$ but not by suckling stimulus $(F(1,16)=0.02347 ; p=0.8802)$, with the number of active neurons elevated on PPD15 and decreasing as the weaning period progressed (Fig. 6a'). Regarding FOS immunoreactivity in central Q2, there was a strong effect of lactation day on this parameter $(F(3,16)=10.75$; $p=0.0004)$, with an overall decreasing trend towards the end of the lactation period (Fig. 6b'). Figure 7 illustrates 


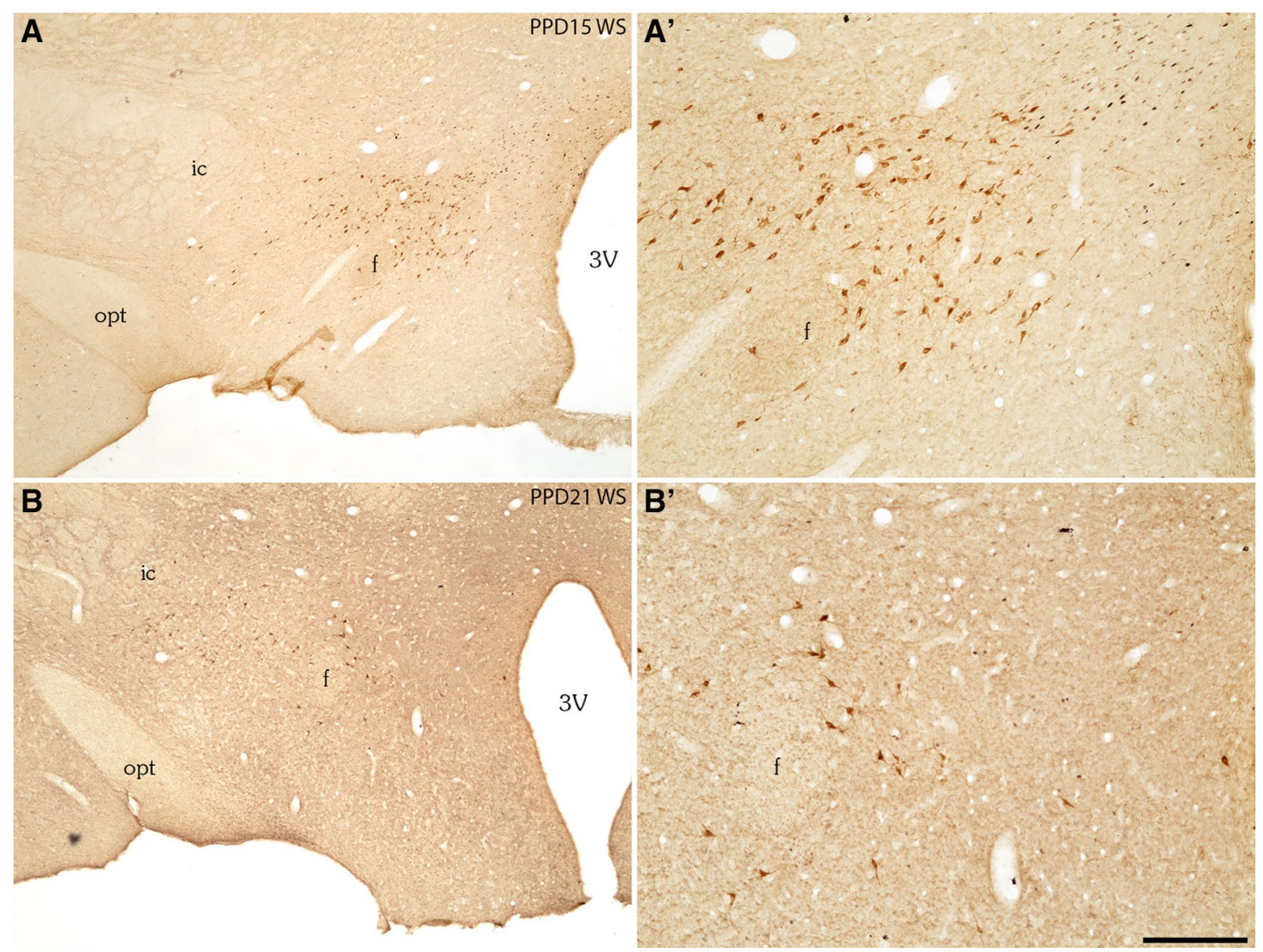

Fig. 4 Orexin-immunoreactive neurons decrease in number in the anterior sector of the tuberal hypothalamus. Brightfield photomicrographs of frontal brain slices from rat dams on the 15th and 21st days of lactation that were subjected to a suckling stimulus. The slices underwent immunostaining for orexin (brown) and FOS (black) localization. a Orexin-immunoreactive neurons are abundantly found in the dorsal aspect of the anterior tuberal hypothalamus at the beginning of the weaning period; $\mathbf{a}^{\prime}$ higher magnification of a illustrat- ing the significant number of immunoreactive neurons in the medial zone of the hypothalamus; $\mathbf{b}$ there is a clear decrease in the number of immunoreactive neurons in the anterior sector of the tuberal hypothalamus of females on the 21 st day of lactation; $\mathbf{b}^{\prime}$ higher magnification of $b$ illustrating that immunoreactive neurons are almost absent from the medial zone. Scale bar: $\mathbf{a}, \mathbf{b}=500 \mu \mathrm{m} ; \mathrm{a}^{\prime}, \mathrm{b}^{\prime}=200 \mu \mathrm{m} .3 \mathrm{~V}$ third ventricle, $f$ fornix, ic internal capsule, opt optic tract the overall distribution of FOS-ir cells in the Q1 and Q2 quadrants over the course of lactation for both stimulated and non-stimulated animals. The number of FOS-ir neurons in central Q3, on the other hand, showed a strong effect of lactation day $(F(3,16)=9.378 ; p=0.0008)$, with an overall decreasing effect as lactation progressed (Fig. $6 c^{\prime}$ ). There was also a trend for the WS group to have elevated numbers of FOS-ir neurons, but the trend did not reach statistical significance $(F(1,16)=4.061$; $p=0.0610$-Fig. $\left.06 \mathrm{c}^{\prime}\right)$. In central $\mathrm{Q} 4$, there were effects of both lactation day $(F(3,16)=4.059 ; p=0.0254)$ and suckling $(F(1,16)=14.90 ; p=0.0014)$ but no interactive effect on the number of FOS-ir neurons (Fig. 6d'). When the groups were individually examined, there was no statistically significant effect of lactation day on the number of FOS-ir neurons in the WS group $(F(3,8)=1.004$; $p=0.4397$ ), while the number of FOS-ir cells in the
NS group was affected by lactation day $(F(3,8)=4.745$; $p=0.0348$-Fig. 6d').

As in the previous sectors, there was a strong effect of lactation day on the number of FOS-ir cells in posterior Q1 $(F(3,16)=16.21 ; p<0.0001)$ and a small interaction factor between lactation day and suckling stimulus $\left(F(3,16)=4.507 ; p=0.0179-F i g\right.$. $\left.6 \mathrm{a}^{\prime \prime}\right)$. The number of FOS-ir cells in posterior Q2 varied according to lactation day $(F(3,16)=4.398 ; p=0.0183)$, but no statistically significant suckling effect was observed (Fig. $\left.6 \mathrm{~b}^{\prime \prime}\right)$. In posterior Q3, there were both lactation day $(F(3,16)=7.813 ; p=0.0017)$ and suckling $(F(1,16)=5.622 ; p=0.0298)$ effects on the number of FOS-ir cells (Fig. $\left.6 \mathrm{c}^{\prime \prime}\right)$. When the groups were individually examined, there was no effect of lactation day on the stimulated group $(F(3,8)=1.109 ; p=0.4005)$, while the opposite was observed for the NS group $(F(3,8)=14.59$; $p=0.0008)$, with a decrease in the number of FOS-ir cells as 


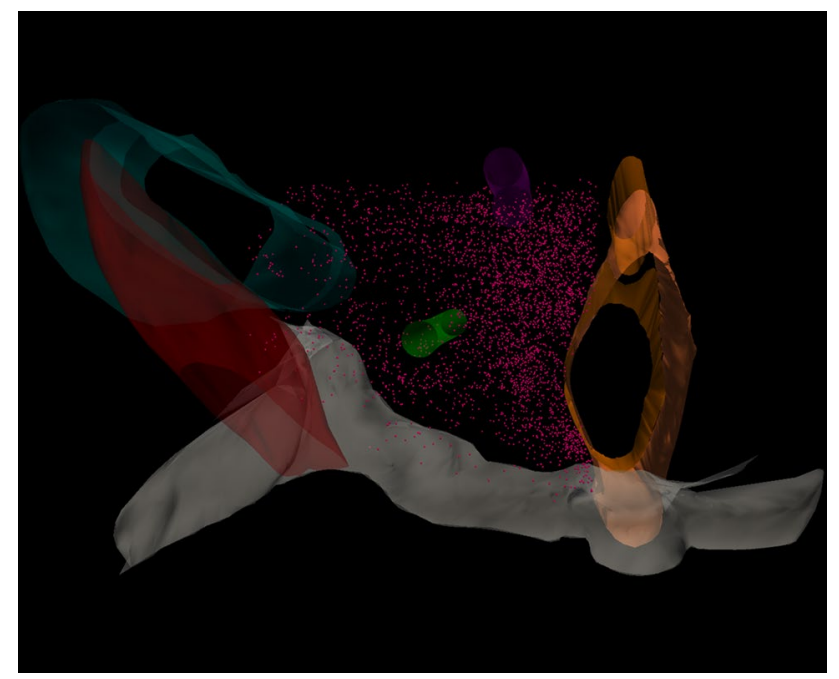

Fig. 5 Distribution of FOS-immunoreactive neurons in the tuberal hypothalamus of a rat dam. Three-dimensional reconstruction based on nine semi-serial frontal slices of the brain of a rat dam on the 15th day of lactation, showcasing the distribution of FOS-immunoreactive cells in the tuberal hypothalamus. Each magenta dot represents a single FOS-immunoreactive cell. The outlined structures are as follows: green-fornix; purple-mammillothalamic tract; orange-third ventricle; dark green-internal capsule/cerebral peduncle; red-optic tract; gray-ventral floor

the weaning period progressed (Fig. $6 \mathrm{c}^{\prime \prime}$ ). Once more, these results indicate that the suckling stimulus stops or at least delays a decrease in activation in Q3. In posterior Q4, there were also lactation day $(F(3,16)=4.566 ; p=0.0160)$ and suckling $(F(1,16)=12.28 ; p=0.0027)$ effects but no interaction (Fig. 6d"). Similar to the previous cases, there was no effect of lactation day on the WS group $(F(3,8)=1.276$; $p=0.3466)$, but there was an effect in the NS group $\left(F(3,8)=5.694 ; p=0.0182\right.$-Fig. $\left.6 \mathrm{~d}^{\prime \prime}\right)$.

\section{Double orexin/FOS-immunoreactive neurons}

Among all sectors, there was a prevalence of double ORX/ FOS-ir neurons in the Q1 quadrant $(85.89 \pm 15.85 \%)$. The preponderance of double-labeled cells in Q1 is illustrated in Fig. 8 (the three-dimensional reconstruction is available in VRML format in Online Resource 5). There was no statistically significant effect of either lactation day or suckling stimulus on the number of double-labeled neurons in any of the other quadrants. Different responses to lactation were observed among sectors. In anterior Q1, there was no effect of either day of lactation or suckling on the number of double ORX/FOS neurons (Fig. 9a). On the other hand, in central Q1, there was a statistically significant main effect of lactation day on the number of those neurons $(F(3,16)=6.623$; $p=0.0041$ ), with an overall decrease in the number of active ORX-ir neurons as lactation progressed (Fig. 9b). When the groups were separately analyzed, the lactation day effect was observable in the WS group $(F(3,8)=4.347 ; p=0.0428)$ but not in the NS group $(F(3,8)=2.498 ; p=0.1337$-Fig. 9b). The number of double-labeled neuron in PPD15 WS was significantly higher than that found on baseline groups $(p<0.05$-Online Resource 2). This effect could not be observed for other lactation days. Although there was no statistically significant difference between the WS and NS groups, there was a trend towards an elevated number of double-labeled cells in the WS group at PPD15 $(p=0.0656)$ that disappeared as lactation progressed. Figure 10 depicts the difference in distribution and number of double ORX/ FOS-ir cells in central Q1 for animals on different lactation days (PPD15 and PPD21) and under different stimulation conditions (WS and NS). In posterior Q1, there was only an effect of lactation day $(F(3,16)=5.137 ; p=0.0104)$ and no clear effect or trend for suckling stimulus (Fig. 9c).

\section{Discussion}

In the present study, we evaluated the relationship between ORX and FOS immunoreactivity in the tuberal hypothalamus of dams during the weaning period, with particular attention to the spatial distribution of labeled cells and the role played by the suckling stimulus from pups. Neurons immunoreactive to ORX were distributed in an ellipsoid shape centered in the tuberal hypothalamus and located dorsally to the fornix, with fewer neurons in the anterior and posterior sectors. Neurons were found in the dorsomedial hypothalamic area, perifornical nucleus and lateral hypothalamic area, as extensively described before (de Lecea et al. 1998; Sakurai et al. 1998). However, the three-dimensional distribution of these cells was much more cohesive, with the ORX population forming a continuous of cells without clear distinctions between populations in different nuclei.

The distribution of ORX-ir neurons in this work parallels that described by Donlin et al. (2014) for the prairie vole, with the highest number of immunoreactive neurons centrally located in the tuberal hypothalamus. In prairie vole dams, there was an increase in the number of ORX-ir cells exclusively in the anterior portion at PPD12, while in our work there was a decrease from PPD15 to PPD21 in rat dams. This led us to hypothesize that this decline was a return to a baseline number of neurons after the lactation period. The number of ORX-ir neurons was higher in PPD15 regardless of suckling stimulus when compared to the baseline groups (Diestrus and Proestrus), but that effect disappeared in the suckling stimulus group, while it was still observed in the PPD21 NS group. Although not statistically significant, the number of neurons in PPD21 WS appeared to be lower than that of the baseline groups, suggesting a small scale rebound effect between the end of lactation and 

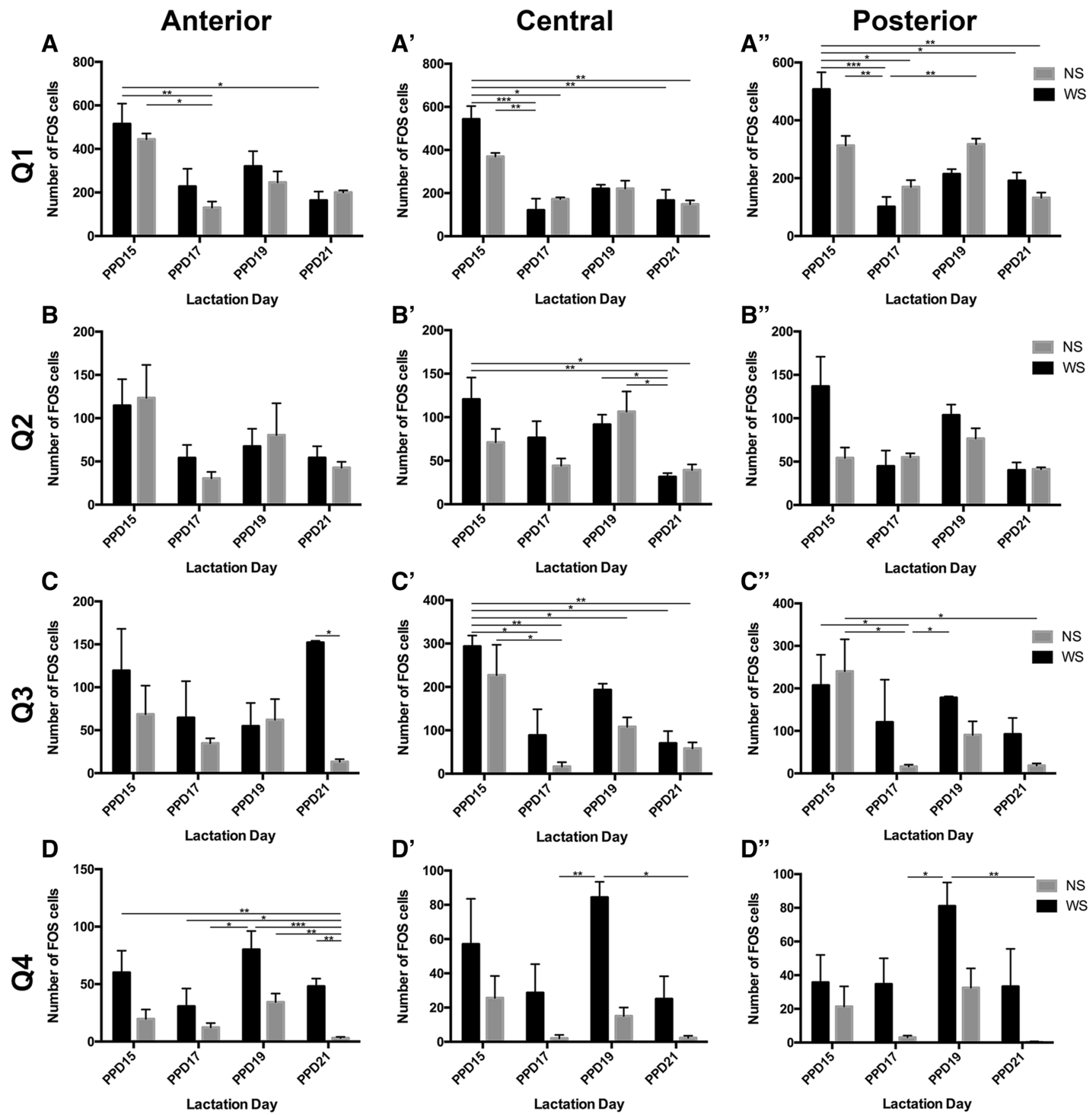

Fig. 6 Variations in the number of FOS-immunoreactive cells for different sectors, quadrants, days of lactation and stimulation conditions. Each bar represents the mean \pm SEM for each day of lactation and stimulation condition. $* p<0.05 ; * * p<0.01 ; * * * p<0.001$

the resume of sexual function. When taken together, these data indicate that an anterior subpopulation of ORX-ir neurons increases in size during early lactation and then shrinks during the weaning period and, furthermore, that this effect is conserved in at least two rodent species with different mating patterns.

Data from other studies in rat dams also support this model. Garcia et al. (2003) detected a decrease in Hcrt
mRNA in dams on PPD14, while Cai et al. (2001) did not detect a lowered level of hypothalamic ORX on the same day. This could indicate that, around PPD14, hypothalamic neurons are preparing to synthesize less ORX, which would be reflected by a decline in ORX immunoreactivity after PPD15, as observed in our work. These results indicate that Hcrt mRNA alterations precede, on the scale of days, any observable alterations in protein synthesis. 
Fig. 7 FOS-immunoreactive cells are unevenly distributed along the lateromedial axis of the tuberal hypothalamus.

Brightfield photomicrographs of frontal brain slices from rat dams on the 15th and 21st days of lactation with and without a suckling stimulus. The slices underwent immunostaining for orexin (brown) and FOS (black) localization. The quadrants employed in this work are delineated with dashed lines. a High number of FOS-immunoreactive cells are found in the periventricular and medial zones of the tuberal hypothalamus, while fewer cells are present in the lateral zone; $\mathbf{b}$ there is a decrease in the number of FOSimmunoreactive neurons in animals that were not stimulated; $\mathbf{c}, \mathbf{d}$ at the end of the weaning period, the density of FOSimmunoreactive cells decreases and almost no difference can be observed between animals with and without the stimulus. Scale bar: $200 \mu \mathrm{m}$. $3 V$ third ventricle, $f$ fornix, ic internal capsule
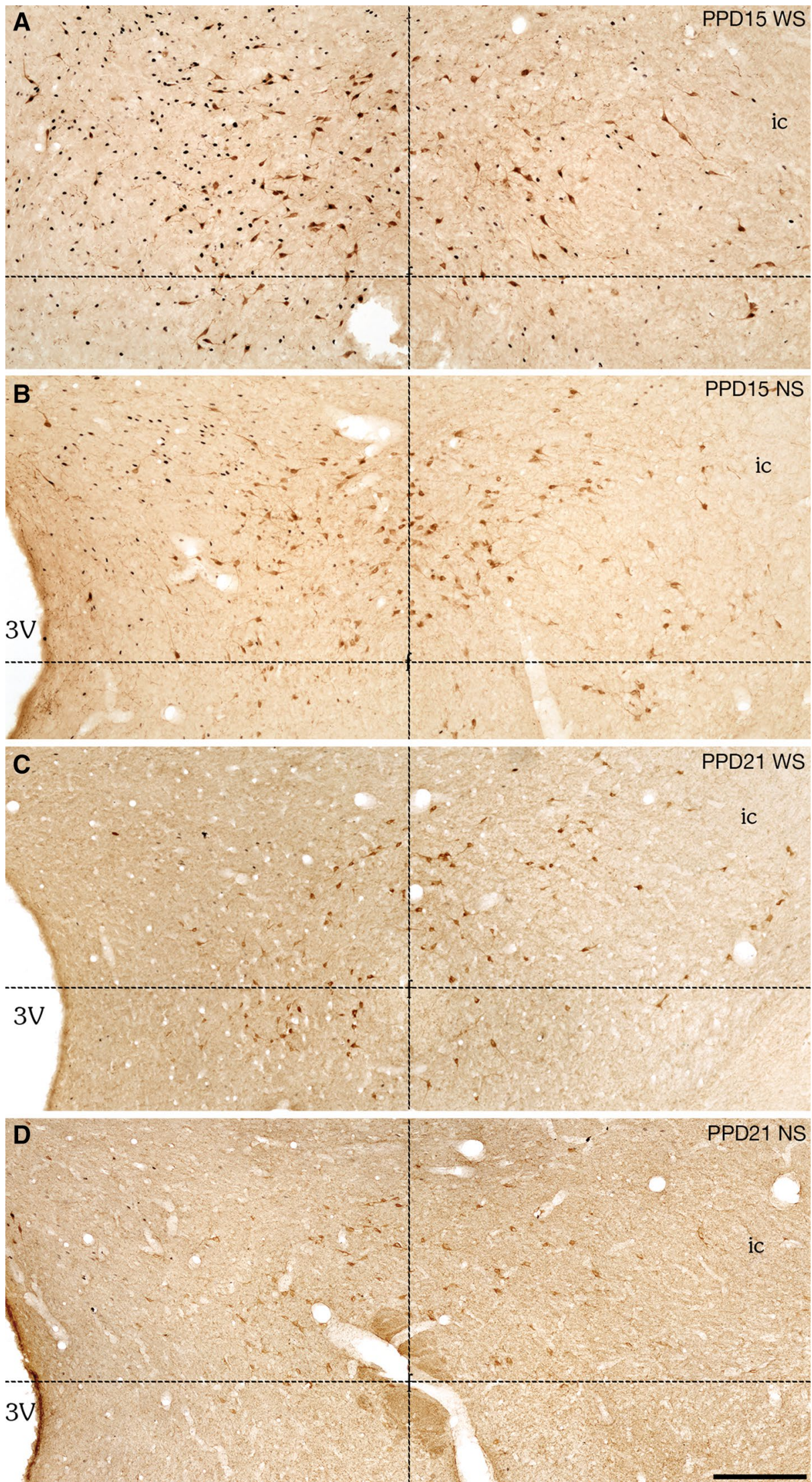


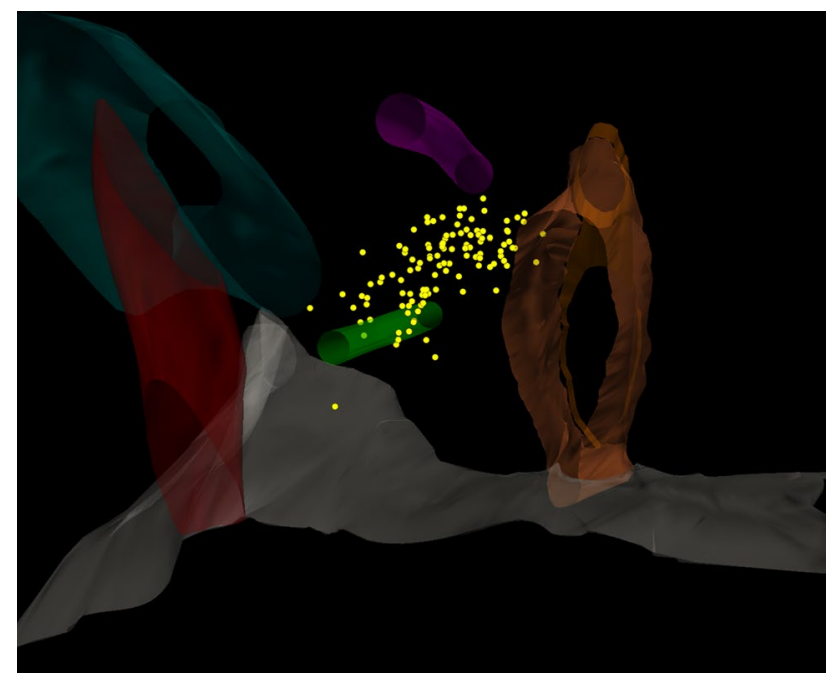

Fig. 8 Distribution of double-labeled orexin/FOS neurons in the tuberal hypothalamus of a rat dam. Three-dimensional reconstruction based on nine semi-serial frontal slices of the brain of a rat dam on the 15th day of lactation, showcasing the distribution of double orexin/FOS-immunoreactive neurons in the tuberal hypothalamus. Each yellow dot represents a single double-labeled cell. The outlined structures are as follows: green-fornix; purple-mammillothalamic tract; orange-third ventricle; dark green-internal capsule/cerebral peduncle; red-optic tract; gray—ventral floor

On earlier days, the reported alterations in ORX synthesis are less clear. Brogan et al. (2000) did not detect any difference in the orexinergic system between PPD10 and virgin animals, while Wang et al. (2003) failed to detect differences in immunoreactivity between PPD1 and PPD14 dams. A possible explanation for this discrepancy is the fact that both Brogan et al. (2000) and Wang et al. (2003) evaluated the hypothalamus as a whole, which may have masked differences occurring exclusively in the anterior subpopulation. Our results support this hypothesis, as the alterations in ORX immunoreactivity were not detectable when the whole hypothalamus was statistically evaluated.

In the central sector of the hypothalamus, the medial zone was unchanged, while the lateral hypothalamus had a small increase in the number of ORX-ir cells as weaning progressed. Sun et al. (2003) examined the central portion of the tuberal hypothalamus and observed an increase in the number of ORX-ir neurons in PPD11-12 dams compared with virgin rats. Together, these results suggest that centrally located ORX-ir neurons are different from the anterior subpopulation concerning their behavior during the lactation period, with the former maintaining higher numbers during the whole lactation period, including the weaning phase, while the anterior population declines in number as weaning progresses.

It is noteworthy that, for both the anterior and the central populations, the suckling stimulus is necessary to sustain the decrease or increase in the number of immunoreactive neurons. This indicates that, even during the weaning period, the suckling stimulus of the pups is still an essential pacemaker of lactation, signaling the progression of this physiological process to the brain of the dam. Perhaps most surprising was the fact that the absence of the suckling stimulus quickly disrupted the progression of alterations in ORX synthesis in the range of hours, on which scale we expected exclusively FOS-related alterations. Merali and Kateb (1993), however, describe bombesin alterations in the hypothalamus of re-fed rats as soon as $35 \mathrm{~min}$, indicating that hypothalamic protein synthesis can be modulated in this short time range. This time course suggests that alterations in the orexin population are caused by the suckling stimulus itself, rather than the larger metabolic state of the animal.

Two possible mechanisms may play a role in this process. The first is direct nipple stimulation from the pups that ascends through the spinal cord, reaches the lateral cervical nucleus and then activates several nuclei in the brainstem (Li et al. 1999). Among these nuclei, a good candidate to
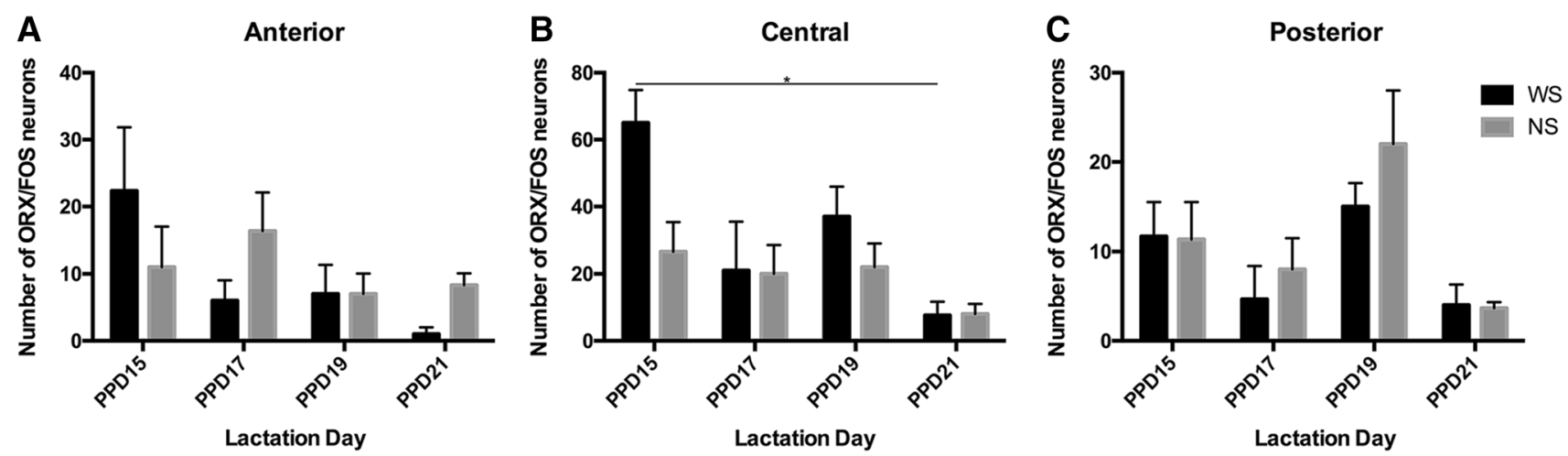

Fig. 9 Variations in the number of double orexin- and FOS-immunoreactive cells in the dorsomedial tuberal hypothalamus for different sectors, days of lactation and stimulation conditions. Each bar represents the mean \pm SEM for each day of lactation and stimulation condition. $* p<0.05$ 
relay signals to orexin cells is the reticular formation, as this area is specifically activated by suckling stimulus ( $\mathrm{Li}$ et al. 1999) and cells in the lateral reticular field are afferents to orexin neurons (Sakurai et al. 2005). In addition to a brainstem-originated input, the tactile stimulus may ascend to the forebrain and then be relayed back to ORX-ir neurons in the hypothalamus, since the ventral lateral septal nucleus and the medial preoptic area are selectively stimulated by suckling and have projections to ORX neurons ( $\mathrm{Li}$ et al. 1999; Sakurai et al. 2005). The second possibility is the activation by oxytocin (OT). Oxytocin depolarizes ORX neurons through receptor V1a (Tsunematsu et al. 2008), and both the supraoptic (SO) and the PVH send afferents to ORX-ir neurons (Sakurai et al. 2005). Even in the absence of direct connections, the somatodendritic release of OT could be responsible for ORX-ir cells activation. Leng and Ludwig (2008) estimate that one minute is sufficient for the anterior hypothalamus to achieve a mean basal concentration of oxytocin in the range of picograms $/ \mathrm{ml}$. That could explain why the anterior and the medial subdivisions of ORX-ir neurons show stronger effects during lactation, as those are the subpopulations that are closer to the $\mathrm{PVH} / \mathrm{SO}$ and, therefore, would be subject to higher concentrations of OT in the extracellular fluid. Although prolactin could also be a factor for the increase in ORX-ir neurons, our data does not support that hypothesis. Rats in the afternoon of the proestrus (high prolactin) had no difference in the number of ORX-ir neurons when compared to rats in the afternoon of the diestrus day (low prolactin).

The alteration in ORX synthesis was the inverse of the null hypothesis of this work: we initially hypothesized that the increase in feeding that dams display during weaning could have as its underlying cause an increase in ORX synthesis or activity. This does not appear to be the case, as there was a decrease in ORX immunoreactivity in the anterior sector and only a small increase in the central lateral zone population as the weaning period progressed. Our results suggest that ORX does not mediate the hyperphagia observed in dams during the later stages of lactation. What role might these neurons play? There are two main possibilities. First, ORX-ir neurons have been thoroughly implicated in the control of sleep-wake patterns, with ORX neurons displaying a potent arousing effect [as reviewed by Sakurai (2007)]. Dams show decreased sleeping time during the lactation period, which España et al. (2004) have suggested to be a result of increased ORX activity. The decline in ORX synthesis in the anterior sector may, therefore, play a major role in the regularization of the sleep cycle of the dam as the pups develop their autonomy.

A second possibility concerns the role of ORX in the expression of maternal behavior, as recent reports have implicated ORX in the expression of these behaviors. D'Anna and Gammie (2006) found an increase in both appetitive and consummatory aspects of maternal behavior after intracerebroventricular injections of low levels of ORXA, while Rivas et al. (2016) found a decrease in appetitive components after injections of an ORX antagonist in the medial preoptic area (MPOA), a brain region thoroughly implicated in maternal behavior (Numan et al. 1977). The declining population of ORX-ir neurons in the anterior part may be particularly involved in maternal behavior, and as the weaning period progresses, this population becomes smaller, since the mother has to display progressively less specific maternal behaviors. One possible approach to distinguish between these two possibilities is to determine the projection field of this anterior ORX-ir population. The work of Rivas et al. (2016) reported that ORX acts on the MPOA to alter maternal behavior, while ORX-ir projections to the tuberomammillary nucleus, locus coeruleus, and dorsal raphe appear to be particularly important for the actions of the peptide on arousal (Sakurai 2007). A selective projection of this group of ORX neurons to some of these areas could point to its main function.

Regarding the suckling-dependent activation of the tuberal hypothalamus, we had previously reported that suckling increased the number of FOS-ir cells in the lateral hypothalamus of dams during the weaning period (Alvisi et al. 2016). In the present work, however, we spatially refined our data on FOS synthesis, showing that there is a preferential activation of the periventricular and medial zones of the hypothalamus compared with the lateral zone. In almost all zones examined, the number of FOS-ir cells decreased as the weaning period progressed, indicating that several neurochemically distinct populations of the tuberal hypothalamus decrease their activity as pups become more independent from the mother. It is noteworthy, however, that the suckling stimulus had an overall effect of increasing the number of FOS-ir cells. This is in stark contrast to the synthesis of ORX, which is actively decreased by the suckling stimulus. Therefore, the suckling stimulus has two different dynamics in the brain of the dam during weaning: it decreases the synthesis of ORX-ir neurons in the anterior sector, and it also decelerates the deactivation of several neuronal populations in the tuberal hypothalamus as weaning progresses. One possible explanation for this apparent contradiction is that ORX represented less than $10 \%$ of the total FOS-ir cell population, suggesting that several other neurochemical populations become less activated during weaning. Some of these populations may have an inhibitory effect on maternal behaviors or other enhanced physiological functions of the dam. Therefore, the suckling stimulus may serve to increase activity in these inhibitory populations and accelerate the cessation of maternal behaviors. Another possibility is that the suckling stimulus may act differently depending on the time range, promoting maternal behaviors in the short term and inhibiting them or restoring them to 

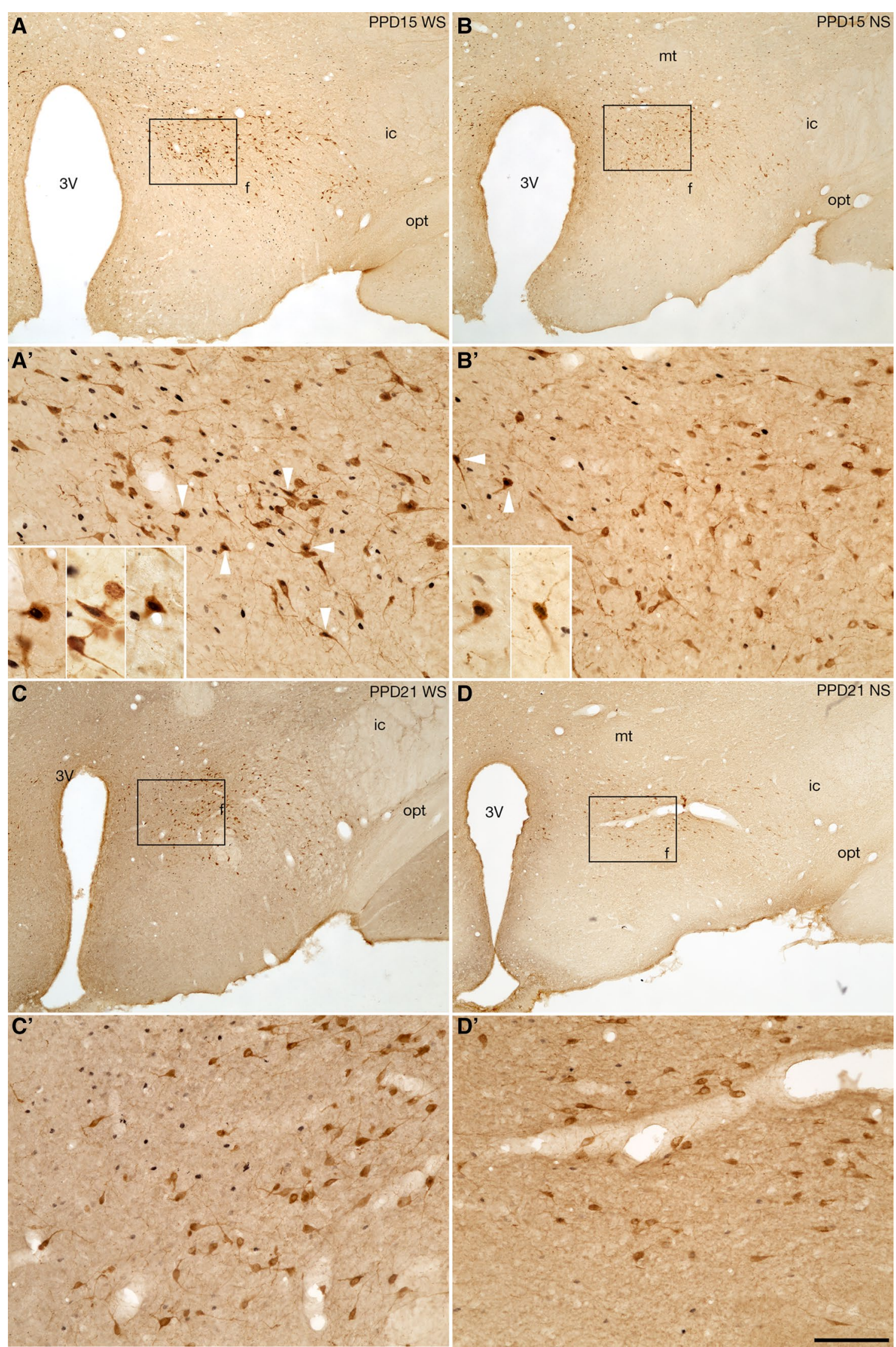
4Fig. 10 Double-labeled orexin/FOS neurons are found in the medial zone of the central tuberal hypothalamus in the early weaning period. Brightfield photomicrographs of frontal brain slices from rat dams on the 15 th and 21 st days of lactation with and without a suckling stimulus. The slices underwent immunostaining for orexin (brown) and FOS (black) localization. a Both orexin- and FOS-immunoreactive neurons are abundant in the medial zone of the tuberal hypothalamus of dams on the 15th day of lactation that were subjected to a suckling stimulus. Several cells contain both markers together, indicating activation of orexin neurons in this region. $\mathbf{a}^{\prime}$ higher magnification of a highlighting some of the double-labeled neurons. Examples of double-labeled cells are present on the inset; $\mathbf{b}$ double-labeled cells can be found in the non-stimulated dams at the 15th day of lactation, although these cells appear to be concentrated close to the periventricular zone; $\mathbf{b}^{\prime}$ higher magnification of $\mathrm{b}$ illustrating the smaller number of double-labeled cells. Examples of double-labeled cells are present on the inset; $\mathbf{c}$ even in stimulated animals, the number of double-labeled cells decreases at the end of the weaning period; $\mathbf{c}^{\prime}$ although both orexin- and FOS-immunoreactive neurons are found in the same field, there is no colocalization of these two markers; $\mathbf{d}$ both double-labeled and FOS-immunoreactive cells are found in decreased numbers in the non-stimulated dams at the 21st day of lactation; $\mathbf{d}^{\prime}$ higher magnification of $\mathbf{d}$. Scale bar: $\mathbf{a}-\mathbf{d}=500 \mu \mathrm{m} ; \mathbf{a}^{\prime}-\mathbf{d}^{\prime}=100 \mu \mathrm{m}$. $3 V$ third ventricle, $f$ fornix, ic internal capsule, opt optic tract

baseline in the long range. Further experiments will be necessary to dissect the role of suckling-activated hypothalamic neurons and to determine the neurochemical signatures of those populations.

Finally, we identified a subpopulation of ORX-ir neurons in the central dorsomedial hypothalamic area that was selectively responsive to the suckling stimulus. Although double ORX/FOS-ir neurons were found in other levels and other quadrants, these neurons were mostly found dorsomedial to the fornix and clustered in the central slices. As lactation progressed, the dams became insensitive to the stimulus and the number of double ORX/FOS-ir neurons equalized between the two groups. Our results agree to the idea of a dichotomy between medial (DMH/PFA) and lateral (LHA) ORX-ir neurons, proposed by Harris and AstonJones (2006). According to this model, medial ORX neurons are implicated in arousal and motivation, while the lateral population has been associated to cue-associated reward. If we transpose our results to this model, the centromedial position of double ORX/FOS-ir neurons suggests that these cells produce increased arousal in the lactating female. One interesting facet of this association is that ORX is not associated to any type of arousal, but to those that require environmental awareness and decision-making, such as exploration and food-shock, but not restriction (Furlong et al. 2009). Considering the unifying theory of ORX as a promoter of motivation (Mahler et al. 2014), the position of our doublelabeled cells suggests that the suckling stimulus activates ORX neurons to promote motivation and awareness in these females. As lactation progresses, however, this activation is lost, as the pups are more independent, and the dam has to be less proactive in their nutrition and protection. This result, along with the previously discussed results, indicates that there is substantial heterogeneity in ORX-ir cells concerning both their input and their behavior during the transitional period of weaning. In particular, a population of dorsomedially located ORX neurons is distinct from other ORX-ir neurons as it shows a sensitivity to the suckling stimulus that may also be associated with differences in hodology and neurochemistry, making this population suited for further studies.

To conclude, our work has important implications for human physiology. It is evident from our results that brain alterations are not exclusive to the peripartum period but also occur during weaning. This weaning period was necessary to decrease ORX-ir synthesis and activation, which may be able to decrease wakefulness time and restore nonlactating patterns of sleep. Perhaps even more important was the fact that the suckling stimulus was necessary to promote these changes. The premature cessation of suckling in humans, therefore, could interrupt the regularization of the orexinergic system, resulting in persistent sleeping problems for the mother. Blyton et al. (2002) have shown that breastfeeding mothers in the postpartum period have different sleep architecture than non-lactating mothers or bottle-feeding mothers: in the breastfeeding mothers, there was a drastic increase in slow-wave sleep (SWS), while bottle-feeding mothers had a decrease in SWS compared with control mothers. Our results suggest, therefore, that breastfeeding may be a major signal that decreases activity in the ORX system and regulates sleeping patterns, meaning that mothers who bottle-feed may have an increased risk of persistent sleeping problems caused by lactation. The ORX system may be an attractive target for evaluation in mothers with postpartum sleep problems.

\section{Conclusions}

Substantial alterations in the orexin peptidergic system occur during the weaning period. A subpopulation of anteriorly located immunoreactive neurons decreases in size as weaning progresses, and this process depends on suckling to occur adequately. The suckling stimulus also increases the number of FOS-ir cells in the tuberal hypothalamus, with a preferential distribution of active cells in the periventricular and medial zones compared with the lateral zone. Finally, a subpopulation of centrally located dorsomedial ORXir neurons is responsive to the suckling stimulus in early weaning but progressively disappears. When taken together, these data suggest that the weaning period is necessary to restore neurochemical pathways altered during the lactation period and that the suckling stimulus plays a major role in this process. 
Acknowledgements This article was supported by Fundação de Amparo à Pesquisa do Estado de São Paulo (São Paulo Research Foundation-FAPESP) grants \#2016/02748-0 (GD), \#2017/17998-5 (MK), \#2010/52068-0, \#2016/02224-1 and \#2017/08908-2 (JB). J-LN and JB are also recipients of a joint Coordenação de Aperfeiçoamento de Pessoal de Nivel Superior (Agency for the Advancement of Higher Education) and Comité Français d'Evaluation de la Coopération Universitaire avec le Brésil (French Committee for the Evaluation of Academic and Scientific Cooperation with Brazil) grant CAPESICOFECUB 848/15. J-LN and FP were supported by the French Centre National de la Recherche Scientifique (The National Center for Scientific Research-CNRS) and the French Agence Nationale de la Recherche (National Research Agency-ANR) through the LABEX SIGNALIFE program \#ANR-11-LABX-0028-01. LF and JB are Investigators with the Conselho Nacional de Desenvolvimento Científico e Tecnológico (National Council for Scientific and Technological DevelopmentCNPq) with grants \#305481/2016-9 (LF) and \#426378/2016-4 (JC).

\section{Compliance with ethical standards}

Conflict of interest The authors declare that this research was performed in the absence of any relationship that could be considered a potential conflict of interest of any kind.

Ethical approval All applicable international, national, and institutional guidelines for the care and use of animals were followed. All procedures performed in studies involving animals were in accordance with the ethical standards of the institution or practice at which the studies were conducted.

\section{References}

Alvisi R, Diniz G, Da-Silva J, Bittencourt J, Felicio L (2016) Suckling-induced Fos activation and melanin-concentrating hormone immunoreactivity during late lactation. Life Sci 148:241-246. https://doi.org/10.1016/j.1fs.2016.02.038

Blyton D, Sullivan C, Edwards N (2002) Lactation is associated with an increase in slow-wave sleep in women. J Sleep Res 11(4):297-303

Branch AF, Navidi W, Tabuchi S, Terao A, Yamanaka A, Scammell TE, Diniz Behn C (2016) Progressive loss of the orexin neurons reveals dual effects on wakefulness. Sleep 39(2):369-377

Brogan R, Grove K, Smith MS (2000) Differential regulation of leptin receptor but not orexin in the hypothalamus of the lactating rat. J Neuroendocrinol 12(11):1077-1086

Burnashev N, Khodorova A, Jonas P, Helm PJ, Wisden W, Monyer H, Seeburg PH, Sakmann B (1992) Calcium-permeable AMPAkainate receptors in fusiform cerebellar glial cells. Science 256(5063): 1566-1570

Cai XJ, Denis R, Vernon RG, Clapham JC, Wilson S, Arch JR, Williams G (2001) Food restriction selectively increases hypothalamic orexin-B levels in lactating rats. Regul Pept 97(2):163-168

Chemelli RM, Willie JT, Sinton CM, Elmquist JK, Scammell T, Lee C, Richardson JA, Williams SC, Xiong Y, Kisanuki Y (1999) Narcolepsy in orexin knockout mice: molecular genetics of sleep regulation. Cell 98(4):437-451

Chen C-T, Dun SL, Kwok EH, Dun NJ, Chang J-K (1999) Orexin A-like immunoreactivity in the rat brain. Neurosci Lett 260(3):161-164

Cramer CP, Thiels E, Alberts JR (1990) Weaning in rats: I. Maternal behavior. Dev Psychobiol 23(6):479-493. https://doi.org/10.1002/ dev.420230604
D’Anna KL, Gammie SC (2006) Hypocretin-1 dose-dependently modulates maternal behaviour in mice. J Neuroendocrinol 18(8):553-566

Date Y, Ueta Y, Yamashita H, Yamaguchi H, Matsukura S, Kangawa K, Sakurai T, Yanagisawa M, Nakazato M (1999) Orexins, orexigenic hypothalamic peptides, interact with autonomic, neuroendocrine and neuroregulatory systems. Proc Natl Acad Sci USA 96(2):748-753

de Lecea L, Kilduff TS, Peyron C, Gao X, Foye PE, Danielson PE, Fukuhara C, Battenberg EL, Gautvik VT, Bartlett FS 2nd, Frankel WN, van den Pol AN, Bloom FE, Gautvik KM, Sutcliffe JG (1998) The hypocretins: hypothalamus-specific peptides with neuroexcitatory activity. Proc Natl Acad Sci USA 95(1):322-327

Donlin M, Cavanaugh BL, Spagnuolo OS, Yan L, Lonstein JS (2014) Effects of sex and reproductive experience on the number of orexin A-immunoreactive cells in the prairie vole brain. Peptides 57:122-128

Edwards C, Abusnana S, Sunter D, Murphy K, Ghatei M, Bloom $S$ (1999) The effect of the orexins on food intake: comparison with neuropeptide $\mathrm{Y}$, melanin-concentrating hormone and galanin. J Endocrinol 160(3):R7-R12

España RA, Berridge CW, Gammie SC (2004) Diurnal levels of Fos immunoreactivity are elevated within hypocretin neurons in lactating mice. Peptides 25(11):1927-1934

Fleming AS (1976) Control of food intake in the lactating rat: Role of suckling and hormones. Physiol Behav 17(5):841-848

Furlong TM, Vianna DM, Liu L, Carrive P (2009) Hypocretin/orexin contributes to the expression of some but not all forms of stress and arousal. Eur J Neurosci 30(8):1603-1614

Garcia MC, Lopez M, Gualillo O, Seoane LM, Dieguez C, Senaris RM (2003) Hypothalamic levels of NPY, MCH, and preproorexin mRNA during pregnancy and lactation in the rat: role of prolactin. FASEB J 17(11):1392-1400. https://doi.org/10.1096/ fj.02-0933com

González JA, Jensen LT, Iordanidou P, Strom M, Fugger L, Burdakov D (2016) Inhibitory interplay between orexin neurons and eating. Curr Biol 26(18):2486-2491

Hagan JJ, Leslie RA, Patel S, Evans ML, Wattam TA, Holmes S, Benham CD, Taylor SG, Routledge C, Hemmati P, Munton RP, Ashmeade TE, Shah AS, Hatcher JP, Hatcher PD, Jones DN, Smith MI, Piper DC, Hunter AJ, Porter RA, Upton N (1999) Orexin A activates locus coeruleus cell firing and increases arousal in the rat. Proc Natl Acad Sci USA 96(19):10911-10916

Harris GC, Aston-Jones G (2006) Arousal and reward: a dichotomy in orexin function. Trends Neurosci 29(10):571-577. https:// doi.org/10.1016/j.tins.2006.08.002

Haynes AC, Jackson B, Overend P, Buckingham RE, Wilson S, Tadayyon M, Arch JR (1999) Effects of single and chronic intracerebroventricular administration of the orexins on feeding in the rat. Peptides 20(9):1099-1105

Ho C-Y, Berridge KC (2013) An orexin hotspot in ventral pallidum amplifies hedonic 'liking'for sweetness. Neuropsychopharmacology 38(9): 1655

Hoffman GE, Le WW, Sita LV (2008) The importance of titrating antibodies for immunocytochemical methods. Curr Protoc Neurosci Chap 2:2-12. https://doi.org/10.1002/0471142301.ns021 $2 s 45$

Kenyon C, Cronin P, Malinek P (1981) Effects of lidocaine on nipple attachment and home orientation by rat pups. Behav Neural Biol 32(2):261-264

Leng G, Ludwig M (2008) Neurotransmitters and peptides: whispered secrets and public announcements. J Physiol 586(23):5625-5632

Li C, Chen P, Smith MS (1999) Neural populations in the rat forebrain and brainstem activated by the suckling stimulus as demonstrated by cFos expression. Neuroscience 94(1):117-129 
Lonstein J, Simmons D, Swann J, Stern J (1997) Forebrain expression of $\mathrm{c}$-fos due to active maternal behaviour in lactating rats. Neuroscience 82(1):267-281

Mahler SV, Aston-Jones GS (2012) Fos activation of selective afferents to ventral tegmental area during cue-induced reinstatement of cocaine seeking in rats. J Neurosci 32(38):13309-13325

Mahler SV, Moorman DE, Smith RJ, James MH, Aston-Jones G (2014) Motivational activation: a unifying hypothesis of orexin/hypocretin function. Nat Neurosci 17(10):1298

Marcondes FK, Bianchi FJ, Tanno AP (2002) Determination of the estrous cycle phases of rats: some helpful considerations. Brazil J Biol 62(4A):609-614

Merali Z, Kateb CC (1993) Rapid alterations of hypothalamic and hippocampal bombesin-like peptide levels with feeding status. Am J Physiol Regul Integr Comp Physiol 265(2):R420-R425

Nambu T, Sakurai T, Mizukami K, Hosoya Y, Yanagisawa M, Goto K (1999) Distribution of orexin neurons in the adult rat brain. Brain Res 827(1):243-260

Numan M, Rosenblatt JS, Komisaruk BR (1977) Medial preoptic area and onset of maternal behavior in the rat. J Comp Physiol Psychol 91(1): 146

Ota K, Yokoyama A (1967) Body weight and food consumption of lactating rats: effects of ovariectomy and of arrest and resumption of suckling. J Endocrinol 38(3):251-261

Paxinos G, Watson C (2013) The Rat Brain in Stereotaxic Coordinates. 7th edn. Academic Press

Peyron C, Tighe DK, van den Pol AN, de Lecea L, Heller HC, Sutcliffe JG, Kilduff TS (1998) Neurons containing hypocretin (orexin) project to multiple neuronal systems. J Neurosci 18(23):9996-10015

Rivas M, Torterolo P, Ferreira A, Benedetto L (2016) Hypocretinergic system in the medial preoptic area promotes maternal behavior in lactating rats. Peptides 81:9-14

Sakurai T (2007) The neural circuit of orexin (hypocretin): maintaining sleep and wakefulness. Nat Rev Neurosci 8(3):171-181

Sakurai T, Amemiya A, Ishii M, Matsuzaki I, Chemelli RM, Tanaka H, Williams SC, Richardson JA, Kozlowski GP, Wilson S (1998) Orexins and orexin receptors: a family of hypothalamic neuropeptides and $\mathrm{G}$ protein-coupled receptors that regulate feeding behavior. Cell 92(4):573-585. https://doi.org/10.1016/S0092 -8674(00)80949-6

Sakurai T, Nagata R, Yamanaka A, Kawamura H, Tsujino N, Muraki Y, Kageyama H, Kunita S, Takahashi S, Goto K (2005) Input of orexin/hypocretin neurons revealed by a genetically encoded tracer in mice. Neuron 46(2):297-308

Saper CB, Swanson LW, Cowan WM (1979) An autoradiographic study of the efferent connections of the lateral hypothalamic area in the rat. J Comp Neurol 183(4):689-706. https://doi.org/10.1002/ cne. 901830402

Sarnat HB, Nochlin D, Born DE (1998) Neuronal nuclear antigen $(\mathrm{NeuN})$ : a marker of neuronal maturation in the early human fetal nervous system. Brain Dev 20(2):88-94

Smith RJ, Aston-Jones G (2012) Orexin/hypocretin 1 receptor antagonist reduces heroin self-administration and cue-induced heroin seeking. Eur J Neurosci 35(5):798-804

Sun G, Narita K, Murata T, Honda K, Higuchi T (2003) Orexin-A immunoreactivity and prepro-orexin mRNA expression in hyperphagic rats induced by hypothalamic lesions and lactation. J Neuroendocrinol 15(1):51-60

Thiels E, Alberts JR, Cramer CP (1990) Weaning in rats: II. Pup behavior patterns. Dev Psychobiol 23(6):495-510. https://doi. org/10.1002/dev.420230605

Tseng M, Chan S-A, Reid K, Iyer V (1997) Fos-like immunoreactivity in rat hippocampal neurons following transient global ischemia. Neurol Res 19(1):89-91

Tsujino N, Sakurai T (2009) Orexin/hypocretin: a neuropeptide at the interface of sleep, energy homeostasis, and reward system. Pharmacol Rev 61(2):162-176

Tsunematsu T, Fu L-Y, Yamanaka A, Ichiki K, Tanoue A, Sakurai T, van den Pol AN (2008) Vasopressin increases locomotion through a V1a receptor in orexin/hypocretin neurons: implications for water homeostasis. J Neurosci 28(1):228-238

Volkoff H, Bjorklund JM, Peter RE (1999) Stimulation of feeding behavior and food consumption in the goldfish, Carassius auratus, by orexin-A and orexin-B. Brain research 846(2):204-209

Wang J, Osaka T, Inoue S (2001) Energy expenditure by intracerebroventricular administration of orexin to anesthetized rats. Neurosci Lett 315(1-2):49-52

Wang J-B, Murata T, Narita K, Honda K, Higuchi T (2003) Variation in the expression of orexin and orexin receptors in the rat hypothalamus during the estrous cycle, pregnancy, parturition, and lactation. Endocrine 22(2):127-134 



\section{Chapter 7}

\section{General Discussion}

To be published in review form as

The neural correlates of weaning: brain plasticity in the post-parental

brain

Diniz GB, Bittencourt JC and Steinbusch HWM 



\subsection{The MCH peptidergic family}

Although the existence of $\mathrm{MCH}$ as a factor that counteracts the actions of a-MSH in the control of skin pigmentation of amphibia has been proposed in the 1930s (Hogben and Slome 1935), almost five decades were necessary for the proper isolation of this peptide from the pituitary of the chum salmon, displaying the exact predicted property of inducing pallor by melanin aggregation (Kawauchi et al. 1983). This discovery paved the way for other researchers to identify the mammalian homologue gene (Nahon et al. 1989) and protein (Vaughan et al. 1989). This ultimately led to the first mapping of MCH in the mammalian brain, by Bittencourt et al. (1992), which unveiled a previously unknown complexity for this peptide.

In the following two decades, the distribution of MCH immunoreactivity has been studied in several species, from some of the most basal chordates (Bird et al. 2001; Mizuno 1991) to humans (Elias et al. 2001). This interest in the various patterns of morphological organization of the MCH peptidergic family stem from the fact that this peptide has been remarkably conserved, and that $\mathrm{MCH}$-synthesizing neurons have widespread projections in the nervous system, what creates an interesting window into the evolution of the vertebrate brain.

Here we reviewed the main morphological aspects of the MCH peptidergic system in mammals, with special attention to the rat, as this species has been the most thoroughly investigated. Such systematic revision is important to highlight the extra-diencephalic areas of synthesis of $\mathrm{MCH}$, since most of the research focus has been directed to the LHA. Although poorly understood, these areas may be part of the anatomical basis for several MCH functions, such as the olfactory tubercle for olfactory integration (Adams et al. 2011), the paramedian pontine reticular formation for sleep and arousal (Costa et al. 2018), and the laterodorsal tegmental nucleus for the sexually dimorphic actions of MCH (Rondini et al. 2007).

The complexity of the diencephalic MCH should not be understated, however. The incertohypothalamic area (IHy) has been recently described (Sita et al. 2003; Sita et al. 2007) and is poorly understood in terms of connectivity and function, even though this area can be found in both rats and humans of both sexes. Perhaps even more interesting is the neurochemical composition of the IHy, with MCH neurons intermingled with dopaminergic neurons, but without co-localization between these two markers. The importance of the contact between these two neurochemical populations is still unknown, but it could make the IHy a site of coordination between the dopaminergic and the MCHergic circuits. In a similar fashion, $\mathrm{MCH}$ neurons in the anterior periventricular nucleus may play an important role in neuroendocrine modulation (Cvetkovic et al. 2003), but the lack of these neurons in mice may be a confounding factor in the study of this particular function. Finally, it is unclear what role may be played by $\mathrm{MCH}$ neurons in the dorsal part of the tuberomammillary nucleus, and if the proximity between $\mathrm{MCH}$ and histamine-positive neurons in this area is indicative of functional significance.

Another contentious point highlighted is the distribution of the MCH receptors, MCHR1 and MCHR2. Based on the available Mchr1 mRNA mappings (Chee et al. 2013; Hervieu et al. 2000; Saito et al. 2001), several areas have conflicting information, regarding both the presence/absence of receptors and the relative density. Additional research is necessary to elucidate the exact distribution of MCHR1 and to expand the physiological models, as only males have been used in these studies. Even more scarce is the available information about MCHR2. This scarcity can be explained by the loss of this receptor subtype in the last common ancestor of the Glires superorder (Tan et al. 2002). This phylogenetic occurrence 
deprived researchers of some of the most commonly used laboratory models for the study of MCHR2, what is reflected in a poor representation of this receptor in the literature. Since this receptor subtype is found in humans, our lack of knowledge about it may help explain our difficulty in transpose functional studies about MCH performed in rodents to human health. It is clear, therefore, that despite extensive research, the morphological aspects of the $\mathrm{MCH}$ peptidergic system are far from being completely understood.

Our next topic of interest was the available functional data and the underlying hodology to support that data. This approach is necessary to better frame MCH actions under a broader physiological context. Our analysis led us to postulate that the main $\mathrm{MCH}$ role in rodents is as a baseline maintainer, a damper that acts to avoid big fluctuations from the desirable homeostatic level. To perform this function, MCH neurons of the LHA receive multiple inputs, including other neurons on the LHA, arcuate and paraventricular nuclei of the hypothalamus, nucleus accumbens, amygdala, brainstem and hindbrain, in addition to circulating factors that reach MCH neurons both directly and indirectly. Drawing from these inputs, MCH neurons are able to monitor the homeostatic status of the organism. At the same time, an equally wide network of outputs allows MCH neurons to regulate metabolic parameters of the organism by changing the locomotor activity, energy expenditure, autonomic function, and thyroid function, by acting as a circulating hormone to act on peripheral MCHR1 receptors, and by modulating reward and foraging.

This framework, therefore, represents a unifying theory for $\mathrm{MCH}$ functions, grouping several of its known actions under the umbrella of homeostatic maintenance. That unifying theory can be expanded to functions not directly linked to energy homeostasis, such as sleep, sexual behavior and maternal behavior. The sleep-promoting activities of MCH may function to counteract the increased arousal that onsets when the animal reaches a negative energy balance, avoiding that too much energy is expended in foraging (Willie et al. 2008). Likewise, MCH may hamper sexual behavior in times of negative energy balance, protecting the female from undergoing the energy-costly process of lactation (Naufahu et al. 2013). Finally, MCH may inhibit maternal behaviors, as demonstrated by Benedetto et al. (2014), returning the mother to her regular physiology and the usual metabolic baseline.

At this point, a brief consideration should be made as to why only $\mathrm{MCH}$, and not the orexins, has been reviewed in this thesis, as they are both the subject of study. First, the distribution of ORX-ir neurons is considerably simpler than that of $\mathrm{MCH}$, forming a continuous of neurons in the medial and lateral hypothalamus, with most cells clustered in the perifornical nucleus (Date et al. 1999; Nambu et al. 1999; Peyron et al. 1998). This distribution was further confirmed by us in three-dimensional reconstructions. This compact distribution makes the morphological data available about ORX less informative than that of $\mathrm{MCH}$. Second, the orexins already have a sound unifying theory of function in the work of Mahler et al. (2014), that proposes ORX as a motivational activator, increasing the overall animal motivation to respond to certain environmental cues depending on its metabolic state.

\subsection{Weaning and the $\mathrm{MCH}$ peptidergic system}

Given the proposed role of MCH as a baseline maintainer, one valuable model to study it is, therefore, the weaning period, due to the severe baseline alterations that occur in this reproductive stage of the female. One could hypothesize that the MCH system will be either overactivated to restore the 
baseline or completely inactivated since the homeostatic balance has been lost and the dam body is operating in non-normal levels of energy storage and activity. That was our objective of study in this thesis. To simplify our study and reduce the number of confounding factors, we emphasized the importance of the pup stimulus to the dams through our experimental designs.

Therefore, we investigated the long-term effects of litter size on the number of MCH neurons in the MPOA of Sprague-Dawley rats. Our results show that large litters are correlated with an increased number of MCH neurons in the MPOA at mid-lactation ( $12^{\text {th }}$ day postpartum), early-weaning $\left(15^{\text {th }}\right.$ day postpartum) and late-weaning (19 th $^{\text {th }}$ day postpartum). Our results further showed that this effect was a combination of both pre- and postpartum litter size, with artificially-reduced litters resulting in an intermediate number of MCH-ir neurons in the MPOA when compared to naturally large or naturally small litters. When taken together with the work of Benedetto et al. (2014), which showed a reduction in appetitive components of maternal behavior by exogenous $\mathrm{MCH}$, our work suggests that the appearance of MCH-ir neurons in the MPOA during late lactation acts as a counterweight to the maternal behaviorinducing stimulus of the pups. The presence of pups is a potent inducer of maternal behavior, even in virgin females (Rosenblatt 1967). Therefore, without a proportional inhibitor, a large litter could drag the lactation to a longer duration, especially if solid food is not easily available to the pups. This lactation extension could be dangerous to the mother, since it would result in increased energy expenditure producing milk, less time to forage and vulnerability to predators, in a time when her fat storages are depleting (Naismith et al. 1982).

Another possibility is that MCH acts on the MPOA to reinitiate sexual behavior by the end of lactation. Previous studies have shown that MCH interacts with GnRH neurons and fibers both in the anteroventral periventricular nucleus and the median eminence to regulate $\mathrm{LH}$ and FSH release (Chiocchio et al. 2001; Gallardo et al. 2004; Gonzalez et al. 1997; Murray et al. 2000; Murray et al. 2006). An increasing synthesis of MCH in the MPOA could help balance the inhibitory effect the pups have on reproductive function (Södersten et al. 1983) and accelerate the resumption of post-weaning sexual function. Regardless of the specific function, we showed that the number of MCH-ir neurons in the MPOA is responsive to litter size during the lactation period, the first demonstration that this synthesis is dependent on exogenous factors.

We also performed a broadly similar examination, but focusing on the acute effect of suckling stimulus on MCH neurons. To achieve that, we employed the widely-used immediate early protein Fos, a marker or neuronal activation (Hoffman et al. 1993). Since Fos is an unspecific marker of activity, studies that use it require a strong, well-defined stimulus to allow discrimination from the background noise. Therefore, instead of culling the litter, we removed the pups at the experimental day, anesthetized the vibrissae pads of the non-stimulated group and returned them to the mother, a protocol based on previous studies (Kenyon et al. 1981; Lonstein et al. 1997). Because of the separation time, pups tended to immediately start suckling on the mothers upon return to the house cage and nursed for the remaining 20 minutes of the experiment. Anesthetized pups, however, were unable to attach to the nipples and, therefore, did not provide the mother with tactile stimulation.

In contrast to our results on the influence of the number of pups on the number of MCH neurons, there was no alteration in the number of $\mathrm{MCH}$-ir neurons in the MPOA of weaning dams linked to suckling stimulus. This is indicative that the number of MCH neurons in the MPOA is dependent on the pup stimulus on a larger time scale, with only chronic stimulation by the pups promoting an increase in the 
number of $\mathrm{MCH}$-ir neurons. Another possibility is that the tactile stimulus is not necessary for the increase in MCH synthesis, with other sensorial modalities fulfilling that role in the non-stimulated groups.

Although the suckling stimulus did not interact with the number of labeled neurons, there was a decrease on the number of MCH-ir neurons in the MPOA in the $20^{\text {th }}$ and $21^{\text {st }}$ days of lactation when compared to the $15^{\text {th }}$ and $16^{\text {th }}$ days. That decrease is in good agreement with data from the previous chapter. Pups in that stage of lactation are quite independent of the mother, and the easiness to obtain solid food accelerates the transition from milk to solid food, reducing the time pups spend nursing (Thiels et al. 1990). It is likely, therefore, that the beginning of weaning represents the apex of MCH synthesis, as from that point onwards the pups will spend progressively less time nursing, and the mother will be less stimulated. This decrease in stimulus must produce the smaller numbers of $\mathrm{MCH}$-ir neurons observed in the $20^{\text {th }}$ and $21^{\text {st }}$ days in this work, and their complete disappearance by the $26^{\text {th }}$ day as reported by Knollema et al. (1992).

Another interesting finding was the complete lack of colocalization between $\mathrm{MCH}$ and Fos. Once more, this result indicates that $\mathrm{MCH}$ plays not the role of an acute responder, but rather that of a much broader integrator of signals and organizer of responses in broader periods of time. Although no colocalization was found between MCH and Fos, numerous Fos-ir cells were found in all three areas examined of stimulated animals, indicating that the experimental approach used was effective in activating suckling-responsive areas. That ample Fos synthesis included the LHA, where numerous stained cells were found intermingled with MCH-ir neurons. Due to the importance of the LHA for the homeostatic balance and the presence of orexin neurons in this area, we decided to investigate if the Fos-ir cells of the LHA could correspond to ORX-ir neurons in the following experiments.

\subsection{Weaning and the ORX peptidergic system}

To investigate if the suckling-stimulated cells of the LHA corresponded to ORX-ir neurons, we employed a similar experimental design for pup stimulus suppression. In our initial work hypothesis, such activation of ORX neurons by the pups could be part of the mechanism that leads to increased food consumption by late lactation, as described by Ota and Yokoyama (1967). As expected, we did find double-labeled ORX/Fos neurons in the tuberal hypothalamus, with a higher number of activated cells found in the $15^{\text {th }}$ day of lactation in the stimulated group when compared to the non-stimulated group or the later stages of weaning.

A striking feature of those active neurons was their location, as over $85 \%$ of those neurons were found dorsomedial to the fornix. To understand this highly specific location of active neurons, one must only turn to the recent literature on ORX function. As proposed by Harris and Aston-Jones (2006), there is a dichotomy between the medial and the lateral orexin neurons. While the medial ORX-ir neurons are implicated in arousal and motivation, the lateral group has been associated with cue-associated reward. Transposing that model to our work, the activation of medial ORX neurons by the suckling stimulus must be part of an arousal-motivation network, increasing the dam's attention to its environment when pups are present and nursing on her. Following that reasoning, the number of active neurons decrease as weaning progresses, since the pups are more independent and, therefore, require less attention from the mother. The fact that only a subset of the neurons in that area were activated by the suckling stimulus 
further reveals the heterogeneity of ORX cells, making this area particularly suitable for further studies of hodology and neurochemistry.

To exam the synthesis of ORX, we first built three-dimensional (3D) models of the immunoreactivity distribution of each animal used in the study. To our best knowledge, these were the first 3D models of the orexin neuronal population published in the literature. Those reconstructions show a very compact population of neurons, forming an ellipsoid dorsal to the fornix, spanning the entire tuberal hypothalamus. The traditional atlas separation of LHA and medial hypothalamus was, therefore, insufficient to accurately describe the anatomical location of labeled neurons. To circumvent that problem, drawing from the work of Harris and Aston-Jones (2006) and Donlin et al. (2014), we devised a system of probes that were placed on the fornix and spliced the tuberal hypothalamus into three sectors: anterior, central and posterior; and four quadrants: dorsomedial, dorsolateral, ventromedial and ventrolateral.

By using this system, we were able to identify a decrease in the number of ORX neurons in the anterior dorsomedial area of the tuberal hypothalamus. This decrease followed the progression of weaning and was disturbed by the loss of suckling stimulation. This led us to hypothesize that suckling stimulus during weaning was responsible for returning the number of anterior ORX neurons in the anterior hypothalamus to baseline levels, what was confirmed when we compared the number of ORX neurons in the $15^{\text {th }}$ day of lactation to those of females during the estrous cycle. As Donlin et al. (2014) found an increase in the number of ORX neurons in the anterior sector of the tuberal hypothalamus of prairie vole dams during mid-lactation, it is likely that this variation in the number of ORX neurons is phylogenetically conserved, at least among rodents. We have confirmed, therefore, the importance of the weaning period to the reconfiguration of the brain neurochemistry to prepartum and pregestational levels.

Perhaps the most important result we obtained, due to its implications to human health, is the fact that the decline in the anterior ORX population was perturbed by the loss of touch stimulation from the pups. Mothers who do not breastfeed, either by choice or by necessity, could be at increased risk of retaining ORX neurons in the anterior dorsomedial hypothalamus, a region associated with the promotion of the awake state and of environmental awareness. Excessive ORX activity in this region could lead to sleep disturbances and emotional alterations, what would negatively impact the health of the mother after the lactation period. Other works have also showed positive effects of breastfeeding for the mother's health, as reviewed in Mohammad and Haymond (2012), and our work provides the neural mechanism for at least some of those benefits. An interesting possibility raised is that mothers with sleep disturbances may be affected by overactivation of the medial ORX population, meaning ORX antagonists already available in the market (Dubey et al. 2015) could be more effective to treat those mothers than broad-effect medication. Further inquiry in this question will be necessary to confirm this possibility.

Finally, we found extensive Fos immunoreactivity linked to the suckling stimulus in the medialmost part of the so-called lateral hypothalamus, an area poorly defined in terms of morphology, neurochemistry and hodology. With ORX-ir neurons accounting for just a small percentage of those Fosir cells, it is likely that several other neuronal populations are modulated during lactation and weaning. This should be taken as further evidence that this period is a prolific field of study, requiring more works to unveil the complete extension of brain alterations that take place in the parental brain. 


\subsection{Conclusion}

In summary, our results demonstrate the intense activity that the brain undergoes during the weaning period. In the medial preoptic area, the number of $\mathrm{MCH}$ neurons that appear in the last two thirds of the lactation period is influenced by the number of pups. In the tuberal hypothalamus, a subset of ORX neurons is stimulated by the tactile stimulus of the pups, at the same time that part of the ORX population recedes. All those changes take place in neuropeptidergic populations widely implicated in essential physiological and behavioral processes, further strengthening the notion that further attention should be payed to the brain alterations that occur in the weaning brain and the repercussions those alterations may have to the mother's health.

\subsection{References}

Adams AC, Domouzoglou EM, Chee MJ, Segal-Lieberman G, Pissios P, Maratos-Flier E (2011) Ablation of the hypothalamic neuropeptide melanin concentrating hormone is associated with behavioral abnormalities that reflect impaired olfactory integration Behav Brain Res 224:195-200

Benedetto L, Pereira M, Ferreira A, Torterolo P (2014) Melanin-concentrating hormone in the medial preoptic area reduces active components of maternal behavior in rats Peptides 58:20-25

Bird DJ, Potter IC, Sower SA, Baker BI (2001) The distribution of melanin-concentrating hormone in the lamprey brain Gen Comp Endocrinol 121:232-241

Bittencourt JC et al. (1992) The melanin-concentrating hormone system of the rat brain: an immuno- and hybridization histochemical characterization J Comp Neurol 319:218-245

Chee MJ, Pissios P, Maratos-Flier E (2013) Neurochemical characterization of neurons expressing melanin-concentrating hormone receptor 1 in the mouse hypothalamus Journal of Comparative Neurology 521:2208-2234

Chiocchio SR, Gallardo MG, Louzan P, Gutnisky V, Tramezzani JH (2001) Melanin-concentrating hormone stimulates the release of luteinizing hormone-releasing hormone and gonadotropins in the female rat acting at both median eminence and pituitary levels Biol Reprod 64:1466-1472

Costa A, Castro-Zaballa S, Lagos P, Chase MH, Torterolo P (2018) Distribution of MCH-containing fibers in the feline brainstem: Relevance for REM sleep regulation Peptides 104:50-61

Cvetkovic V, Brischoux F, Griffond B, Bernard G, Jacquemard C, Fellmann D, Risold PY (2003) Evidence of melanin-concentrating hormone-containing neurons supplying both cortical and neuroendocrine projections Neuroscience 116:31-35

Date $Y$ et al. (1999) Orexins, orexigenic hypothalamic peptides, interact with autonomic, neuroendocrine and neuroregulatory systems Proc Natl Acad Sci U S A 96:748-753

Donlin M, Cavanaugh BL, Spagnuolo OS, Yan L, Lonstein JS (2014) Effects of sex and reproductive experience on the number of orexin A-immunoreactive cells in the prairie vole brain Peptides 57:122-128

Dubey AK, Handu SS, Mediratta PK (2015) Suvorexant: the first orexin receptor antagonist to treat insomnia Journal of pharmacology \& pharmacotherapeutics 6:118

Elias CF, Lee CE, Kelly JF, Ahima RS, Kuhar M, Saper CB, Elmquist JK (2001) Characterization of CART neurons in the rat and human hypothalamus I Comp Neurol 432:1-19

Gallardo MG, Chiocchio SR, Tramezzani JH (2004) Changes of melanin-concentrating hormone related to LHRH release in the median eminence of rats Brain Res 1030:152-158

Gonzalez MI, Baker BI, Wilson CA (1997) Stimulatory effect of melanin-concentrating hormone on luteinising hormone release Neuroendocrinology 66:254-262

Harris GC, Aston-Jones G (2006) Arousal and reward: a dichotomy in orexin function Trends Neurosci 29:571-577

Hervieu GJ, Cluderay JE, Harrison D, Meakin J, Maycox P, Nasir S, Leslie RA (2000) The distribution of the mRNA and protein products of the melanin-concentrating hormone $(\mathrm{MCH})$ receptor gene, slc-1, in the central nervous system of the rat Eur J Neurosci 12:1194-1216

Hoffman GE, Smith MS, Verbalis JG (1993) c-Fos and related immediate early gene products as markers of activity in neuroendocrine systems Frontiers in neuroendocrinology 14:173-213

Hogben L, Slome D (1935) The Pigmentary Effector System. VIII.--The Dual Receptive Mechanism of the Amphibian Background Response vol 120. Proceedings of the Royal Society of London. Series B, Biological Sciences, vol 817. The Royal Society.

Kawauchi H, Kawazoe I, Tsubokawa M, Kishida M, Baker BI (1983) Characterization of melanin-concentrating hormone in chum salmon pituitaries Nature 305:321-323 
Kenyon C, Cronin P, Malinek P (1981) Effects of lidocaine on nipple attachment and home orientation by rat pups Behavioral and neural biology 32:261-264

Knollema S, Brown ER, Vale W, Sawchenko PE (1992) Novel hypothalamic and preoptic sites of prepro-melanin-concentrating hormone messenger ribonucleic Acid and Peptide expression in lactating rats J Neuroendocrinol 4:709-717

Lonstein J, Simmons D, Swann J, Stern J (1997) Forebrain expression of c-fos due to active maternal behaviour in lactating rats Neuroscience 82:267-281

Mahler SV, Moorman DE, Smith RJ, James MH, Aston-Jones G (2014) Motivational activation: a unifying hypothesis of orexin/hypocretin function Nature neuroscience 17:1298

Mizuno N (1991) Electron microscopic identification of axons containing melanin-concentrating hormone in the lamprey, Lampetra fluviatilis L Neuroscience letters 128:249-252

Mohammad MA, Haymond MW (2012) The magic of mother's milk Diabetes 61:3076-3077

Murray JF et al. (2000) Melanin-concentrating hormone, melanocortin receptors and regulation of luteinizing hormone release J Neuroendocrinol 12:217-223

Murray JF et al. (2006) Evidence for a Stimulatory Action of Melanin-Concentrating Hormone on Luteinising Hormone Release Involving MCH1 and Melanocortin-5 Receptors Journal of neuroendocrinology 18:157-167

Nahon JL, Presse F, Bittencourt JC, Sawchenko PE, Vale W (1989) The rat melanin-concentrating hormone messenger ribonucleic acid encodes multiple putative neuropeptides coexpressed in the dorsolateral hypothalamus Endocrinology 125:2056-2065

Naismith D, Richardson D, Pritchard AE (1982) The utilization of protein and energy during lactation in the rat, with particular regard to the use of fat accumulated in pregnancy British journal of nutrition 48:433-441

Nambu T, Sakurai T, Mizukami K, Hosoya Y, Yanagisawa M, Goto K (1999) Distribution of orexin neurons in the adult rat brain Brain research 827:243-260

Naufahu J, Cunliffe AD, Murray JF (2013) The roles of melanin-concentrating hormone in energy balance and reproductive function: Are they connected? Reproduction 146:R141-150

Ota K, Yokoyama A (1967) Body weight and food consumption of lactating rats: effects of ovariectomy and of arrest and resumption of suckling Journal of Endocrinology 38:251-261

Peyron C, Tighe DK, van den Pol AN, de Lecea L, Heller HC, Sutcliffe JG, Kilduff TS (1998) Neurons containing hypocretin (orexin) project to multiple neuronal systems J Neurosci 18:9996-10015

Rondini TA, Rodrigues Bde C, de Oliveira AP, Bittencourt JC, Elias CF (2007) Melanin-concentrating hormone is expressed in the laterodorsal tegmental nucleus only in female rats Brain Res Bull 74:21-28

Rosenblatt JS (1967) Nonhormonal basis of maternal behavior in the rat Science 156:1512-1513

Saito Y, Cheng M, Leslie FM, Civelli O (2001) Expression of the melanin-concentrating hormone (MCH) receptor mRNA in the rat brain J Comp Neurol 435:26-40

Sita LV, Elias CF, Bittencourt JC (2003) Dopamine and melanin-concentrating hormone neurons are distinct populations in the rat rostromedial zona incerta Brain Res 970:232-237

Sita LV, Elias CF, Bittencourt JC (2007) Connectivity pattern suggests that incerto-hypothalamic area belongs to the medial hypothalamic system Neuroscience 148:949-969

Södersten P, Hansen S, Eneroth P (1983) Inhibition of sexual behaviour in lactating rats Journal of endocrinology 99:189-197

Tan CP et al. (2002) Melanin-concentrating hormone receptor subtypes 1 and 2: species-specific gene expression Genomics 79:785-792

Thiels E, Alberts JR, Cramer CP (1990) Weaning in rats: II. Pup behavior patterns Developmental psychobiology 23:495-510

Vaughan JM, Fischer WH, Hoeger C, Rivier J, Vale W (1989) Characterization of melanin-concentrating hormone from rat hypothalamus Endocrinology 125:1660-1665

Willie JT, Sinton CM, Maratos-Flier E, Yanagisawa M (2008) Abnormal response of melanin-concentrating hormone deficient mice to fasting: hyperactivity and rapid eye movement sleep suppression Neuroscience 156:819-829 

Appendix A Summary [English] 



\section{Summary [English]}

Lactation, one of the defining characteristics of the Mammalia phylum, is an effective reproductive strategy that maximizes the survival of the litter at the expense of the mother own energy reserves. To achieve that, the dam physiology undergoes several transformations that will maximize her energy acquisition and storage. To organize those adaptations, the brain also undergoes several modifications. Once the pups transition to a solid-diet independent from the mother, weaning starts and the dam physiology must be restored to pre-gestational levels to allow a new reproductive cycle to start. Little is understood about how the brain copes with those changes and the return to normal physiology, with potential negative effects resulting from an incomplete process. In light of those facts, we proposed with this work to investigate the influence of the suckling stimulus on the lateral hypothalamus during weaning, with special attention to the melanin-concentrating hormone $(\mathrm{MCH})$ and orexin (ORX) populations, two neuropeptidergic populations that have been implicated in several essential physiological and behavioral processes.

In Chapter 1, a more extensive introduction about the parental brain and the importance of the MCH and ORX populations is presented to the reader. In Chapter 2, we review the major morphological aspects of the MCH system, including all peptides synthesized from the MCH precursor, and its receptors. The neuroanatomy of the system is evaluated in depth, including detailed descriptions of the immunoreactive cell bodies, fibers and receptors in the mammalian brain, and the need for studies in new animal models is highlighted. In Chapter 3 we review the major functions associated to MCH and the underlying connectivity. We propose in this review that most MCH functions can be understood as a single process: the maintenance of the homeostatic baseline by $\mathrm{MCH}$, which works as a dampener to prevent possibly deleterious fluctuations in metabolic parameters.

In Chapter 4, we investigate the effect of litter size on the MPOA population of MCH neurons. To that end, mothers were separated in two groups according to the size of their naturally-occurring litter and the number of $\mathrm{MCH}$-immunoreactive neurons was evaluated at mid-lactation, early weaning and late weaning. Our results show that the number of immunoreactive neurons in the MPOA was directly correlated to the size of the litter at all time points studied. Considering the available literature, our results seem to suggest that MCH synthesis in the MPOA works as a counterweight to the maternal-inducing stimulus of the pups. Artificial culling of the litter after birth revealed that this effect has both a pre- and a post-partum component.

In Chapter 5, we investigate the acute effects of the suckling stimulus on the activation of MCH neurons in the whole hypothalamus of weaning rat dams. To suppress the suckling stimulus, a group of pups had their vibrissae pads anesthetized and were allowed to return to the home cage. The brain of the dams was then examined for the presence of the Fos protein in addition to MCH immunoreactivity. Although widespread Fos immunoreactivity was found in the hypothalamus of the weaning dams, there was no colocalization between that marker and $\mathrm{MCH}$, indicating that the acute suckling stimulus cannot alone recruit the $\mathrm{MCH}$ peptidergic system. Furthermore, we found no alteration in the number of $\mathrm{MCH}$ neurons in the MPOA linked to the short-term pup stimulus.

In Chapter 6, we investigate if the Fos immunoreactivity found in the tuberal hypothalamus of weaning dams was indicative of the recruitment of the orexin neuronal population present in this area. Three-dimensional reconstructions were employed to answer that question. We found a small but well- 
defined population of ORX-immunoreactive neurons in the dorsomedial tuberal hypothalamus of the weaning dam that was activated by the suckling stimulus. In addition to this dorsomedial population, we discovered that the weaning period resulted in a reduction in the number of ORX-immunoreactive neurons in the anterior sector of the tuberal hypothalamus, and that reduction was perturbed by the loss of suckling stimulus. Considering the role of the medial ORX neurons to environmental awareness and attention, our results suggest that the weaning period is necessary to normalize the action of ORX neurons, and the lack of sensorial stimulation from the pups can potentially have deleterious effects. Altogether, our results show a remarkable heterogeneity among ORX-immunoreactive neurons of the tuberal hypothalamus. In Chapter 7, we discuss the main results found in the aforementioned works, with a focus on the perspectives in the field.

In summary, our results demonstrate the intense activity that the brain undergoes during the weaning period. In the medial preoptic area, the number of $\mathrm{MCH}$ neurons that appear in the last two thirds of the lactation period is influenced by the number of pups. In the tuberal hypothalamus, a subset of ORX neurons is stimulated by the tactile stimulus of the pups, at the same time that part of the ORX population recedes. All those changes take place in neuropeptidergic populations widely implicated in essential physiological and behavioral processes, further strengthening the notion that further attention should be payed to the brain alterations that occur in the weaning brain and the repercussions those alterations may have to the mother's health. 


\section{Appendix B Summary [Dutch]}





\section{Summary [Dutch]}

Melkafscheiding, een van de bepalende kenmerken van het fylum Mammalia, is een effectieve reproductiestrategie die het voortbestaan van het nest maximaliseert ten koste van de eigen energiereserves van de moeder. Om dit te bereiken, ondergaat de fysiologie van de moeder verschillende transformaties die haar energie-acquisitie en opslag maximaliseren. Om die aanpassingen te organiseren, ondergaat het brein ook verschillende aanpassingen. Zodra de jongen overgaan op een vast dieet onafhankelijk van de moeder, begint het spenen en moet de moederfysiologie worden hersteld tot het niveau van vóór de zwangerschap om een nieuwe voortplantingscyclus te starten. Er is weinig bekend over hoe het brein omgaat met die veranderingen en de terugkeer naar de normale fysiologie, met mogelijke negatieve effecten als gevolg van een onvolledig proces. In het licht van deze feiten, hebben we met dit onderzoek voorgesteld om de invloed van de zuigstimulus op de laterale hypothalamus tijdens het spenen te onderzoeken, met speciale aandacht voor de melanine-concentrerende hormoon $(\mathrm{MCH})$ en orexin (ORX) populaties, twee neuropeptidergische populaties die betrokken geweest bij verschillende essentiële fysiologische en gedragsprocessen.

In Hoofdstuk 1 wordt een uitgebreidere inleiding over het ouderlijk brein en het belang van de MCH- en ORX-populaties aan de lezer gepresenteerd. In Hoofdstuk 2 bespreken we de belangrijkste morfologische aspecten van het MCH-systeem, inclusief alle peptiden gesynthetiseerd uit de $\mathrm{MCH}$ precursor en zijn receptoren. De neuroanatomie van het systeem wordt diepgaand geëvalueerd, inclusief gedetailleerde beschrijvingen van de immunoreactieve cellichamen, vezels en receptoren in de hersenen van zoogdieren, en de behoefte aan studies in nieuwe diermodellen wordt benadrukt. In Hoofdstuk 3 bespreken we de belangrijkste functies die horen bij MCH en de onderliggende connectiviteit. We stellen in deze recensie voor dat de meeste MCH-functies kunnen worden begrepen als één enkel proces: het onderhouden van de homeostatische basislijn door $\mathrm{MCH}$, dat werkt als een demper om mogelijk schadelijke fluctuaties in metabole parameters te voorkomen.

In Hoofdstuk 4 onderzoeken we het effect van worpgrootte op de MPOA-populatie van MCHneuronen. Daartoe werden moeders in twee groepen gescheiden op basis van de grootte van hun in de natuur voorkomende nest en het aantal MCH-immunoreactieve neuronen werd geëvalueerd halverwege de lactatie, vroeg spenen en laat spenen. Onze resultaten laten zien dat het aantal immunoreactieve neuronen in de MPOA op alle onderzochte tijdstippen direct verband hield met de grootte van het nest. Gezien de beschikbare literatuur lijken onze resultaten erop te wijzen dat de $\mathrm{MCH}$ synthese in de MPOA werkt als een tegengewicht voor de moederinducerende stimulus van de jongen Het kunstmatig ruimen van het nest na de geboorte onthulde dat dit effect zowel een pre- als een postpartumcomponent heeft.

In Hoofdstuk 5 onderzoeken we de acuut effecten van de zuigstimulus op de activatie van MCHneuronen in de hele hypothalamus van speendammen. Om de zuigstimulus te onderdrukken, liet een groep jongen hun snoet verdoven en mochten ze terugkeren naar de kooi. De hersenen van de moederdieren werden vervolgens onderzocht op de aanwezigheid van het Fos-eiwit naast de immunoreactiviteit van MCH. Hoewel wijdverspreide immunoseactiviteit van Fos werd gevonden in de hypothalamus van de speendammen, was er geen colocalisatie tussen die marker en $\mathrm{MCH}$, wat aangeeft dat de acute zuigstimulus niet alleen het MCH peptidergische systeem kan aanwerven. Verder vonden 
we geen verandering in het aantal MCH-neuronen in de MPOA gekoppeld aan de korte-termijnpupenstimulus.

In Hoofdstuk 6 onderzoeken we of de immunoreactiviteit van Fos gevonden in de tuberale hypothalamus van speendammen indicatief was voor de rekrutering van de orexine neuronale populatie die aanwezig is in dit gebied. Driedimensionale reconstructies werden gebruikt om die vraag te beantwoorden. We vonden een kleine maar goed gedefinieerde populatie van ORX-immunoreactieve neuronen in de dorsomediale tuberale hypothalamus van de speendam die werd geactiveerd door de zuigstimulus. Naast deze dorsomediale populatie, ontdekten we dat de speenperiode resulteerde in een vermindering van het aantal ORX-immuunreactieve neuronen in de anterieure sector van de tuberale hypothalamus, en die vermindering werd verstoord door het verlies van zuigstimulus. Gezien de rol van de mediale ORX-neuronen voor het milieubewustzijn en de aandacht suggereren onze resultaten dat de speenperiode nodig is om de werking van ORX-neuronen te normaliseren, en het gebrek aan sensoriële stimulatie van de pups kan mogelijk schadelijke effecten hebben. Al met al laten onze resultaten een opmerkelijke heterogeniteit zien tussen ORX-immunoreactieve neuronen van de tuberale hypothalamus. In Hoofdstuk 7 bespreken we de belangrijkste resultaten in de bovengenoemde werken, met een focus op de perspectieven in het veld.

Samengevat tonen onze resultaten de intense activiteit aan die de hersenen ondergaan tijdens de speenperiode. In het mediale preoptische gebied wordt het aantal MCH-neuronen dat in de laatste twee derde van de lactatieperiode voorkomt, beïnvloed door het aantal jongen. In de tuberale hypothalamus wordt een subset van ORX-neuronen gestimuleerd door de tactiele stimulus van de jongen, op hetzelfde moment dat een deel van de ORX-populatie terugvalt. Al deze veranderingen vinden plaats in neuropeptidergroepen die op grote schaal betrokken zijn bij essentiële fysiologische en gedragsprocessen, wat het idee versterkt dat er verdere aandacht moet worden besteed aan de hersenveranderingen die optreden in het speenbrein en de gevolgen die deze veranderingen kunnen hebben voor de gezondheid van de moeder. 
Appendix C Valorisation 



\section{Valorisation}

The lactation period is perhaps unrivaled in terms of the extension and depth of the metabolic demands that it imposes on the female during a short period of time. This makes motherhood a very attractive field of research, as it equips researchers with a physiological model of brain plasticity in response to both internal and external cues. An often-overlooked aspect of lactation, however, is weaning, or the terminus of lactation. During weaning, the brain must undergo a new set of alterations that will reset most of its functions to their pre-pregnancy and pre-lactation levels. The inexorable growth of the pups exerts a strong pressure on the dam's body to constrain its duration and ensure a swift interruption of maternal behaviors and resuming of reproductive functions.

If examined out of context, several of the brain alterations that take place in the maternal physiology would be considered pathological at any other moment of the dam's life. Those alterations include, for example, leptin resistance, one of the hallmarks of metabolic syndrome, decreased (and therefore potentially insufficient) response to stressors, and excessive volume expansion at the cost of harshly reduced osmoregulation. It is logical, therefore, that if weaning does not proceed appropriately, the once-mother could carry unbalances for an unknown extension of time. To identify and understand the weaning alterations in the central nervous system, therefore, could provide us with the knowledge to better intervene if something goes awry during this period.

Although an underappreciated problem, motherhood can be challenging for several women and may leave long-standing consequences. Excessive weight gain during lactation is a predictor of obesity and cardiovascular risk decades after birth (Mannan et al. 2013; McClure et al. 2013). Sleep patterns are altered during pregnancy and lactation and there is no conclusive evidence about when and if the sleep patterns return to the baseline levels (Lee 1998). Postpartum depression is a crippling illness for mothers, that could be as prevalent as 30\% depending on the criteria used (Brummelte and Galea 2016). All those problems have poorly-understood causes, what makes studies focused on the brain processes of lactation and weaning particularly important to start unveiling the mechanisms that underlie those conditions.

Therefore, the results of the presented dissertations should be of high interest to all professionals in the medical field that work with women's health, especially those in the obstetrics field. Our work also fulfills a broader role of highlighting particular processes that occur in the female brain, an important step to correct the chronic underrepresentation of female subjects in research (Prendergast et al. 2014). Finally, our results should be of interest to the pharmacy industry, as we have identified a group of individuals (mothers who bottle feed) as under increased risk of inefficient brain restauration of circuits controlling sleep architecture and awareness, and we identified the neurochemical group that may be targeted for pharmacological intervention to restore the normal brain function. Clinical trials could be performed comparing the efficiency of orexin antagonism-based medication (Dubey et al. 2015) against other types of medication used in the treatment of insomnia in women in the postpartum period. Those results could then be used to inform public policies about the treatment of sleep disturbances in adult women.

Regarding the innovation of the presented results, this work can be considered innovation as it tackles a poorly-explored experimental model: the weaning brain. While most works investigated the mother physiology and behavior a few days after birth, only a few works have investigated what happens 
to the dam body as lactation comes to an end. This has direct market consequences, as medical products directed to the mother's health after delivery are minimal in comparison to those marketed for mothers during gestation or to the health of the babies. An example of this is the lack of any medication to target postpartum depression, the most common complication of childbearing, even though it is believed that postpartum depression shares only a part of its etiology with other depression disorders (Wisner et al. 2002).

To conclude, this dissertation is of relevance not only to the academic field, but also to the medical field of obstetrics and, particularly, of postpartum health. It may also lead to further research into the targeting of orexin for the treatment of sleep disorders in mothers, what could help inform public policies of women's health. We believe our results may have an important positive impact for society as a whole.

\section{References}

Brummelte S, Galea LA (2016) Postpartum depression: etiology, treatment and consequences for maternal care Hormones and behavior 77:153-166

Dubey AK, Handu SS, Mediratta PK (2015) Suvorexant: the first orexin receptor antagonist to treat insomnia Journal of pharmacology \& pharmacotherapeutics 6:118

Lee KA (1998) Alterations in sleep during pregnancy and postpartum: a review of 30 years of research Sleep medicine reviews 2:231-242

Mannan M, Doi SA, Mamun AA (2013) Association between weight gain during pregnancy and postpartum weight retention and obesity: a bias-adjusted meta-analysis Nutrition reviews 71:343-352

McClure CK, Catov JM, Ness R, Bodnar LM (2013) Associations between gestational weight gain and BMI, abdominal adiposity, and traditional measures of cardiometabolic risk in mothers 8 y postpartum- The American journal of clinical nutrition 98:1218-1225

Prendergast BJ, Onishi KG, Zucker I (2014) Female mice liberated for inclusion in neuroscience and biomedical research Neuroscience \& Biobehavioral Reviews 40:1-5

Wisner KL, Parry BL, Piontek CM (2002) Postpartum depression New England Journal of Medicine 347:194-199 
Appendix

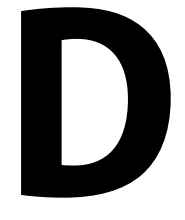

About the Author 



\section{Curriculum Vitae}

Giovanne B. Diniz was born on $8^{\text {th }}$ day of September, 1992, in São Paulo, Brazil. His primary education was completed in São Paulo in 2010, when he joined the Undergraduate Course in Biosciences of the University of São Paulo at the age of seventeen. In the same year, he applied to and was approved to be transferred to the Undergraduate Course of Molecular Sciences of the University of São Paulo.

During his undergraduate education, he joined the laboratory of Prof. Jackson C. Bittencourt to study the actions of melanin-concentrating hormone in the lactating brain. In $2013 \mathrm{Mr}$. Diniz received a Travel Award from the University of São Paulo to attend the "Genes, Circuits and Behavior" Symposia in Toronto, Canada, and present his work. During this period, he met Prof. Jean-Louis Nahon and became an intern at his lab in Sophia-Antipolis, France, for three months tanks to an Academic Merit Award from the University of São Paulo. In August 2014 he completed his undergraduate course, receiving from the University of São Paulo the title of Bachelor of Science. In the same year, he joined the Master's Degree program on Morphofunctional Sciences of the Institute of Biomedical Sciences of the University of São Paulo. After his qualifying examinations, in 2015, his evaluation committee recommended that Mr. Diniz should switch to the Direct Doctorate program at the same Institution.

In 2016, Mr. Diniz was selected to participate on the Society for Neuroscience's (SfN) Latin American Training Program School, held in Montevideo, Uruguay with financing from SfN, the International Brain Research Organization (IBRO), and the Grass Foundation. In 2016 he also spent two weeks in Prof. Paul Sawchenko's Lab at the Salk Institute (La Jolla, California) as a Research Collaborator to learn cold-probe methods of in situ hybridization and to attend the SfN Meeting held in that year.

In 2017, Prof. Harry Steinbusch visited the Laboratory of Prof. Bittencourt at the University of São Paulo, and talks began for Mr. Diniz to apply for a double-degree between the University of São Paulo and Maastricht University. He attended the SfN Meeting held in 2017 at Washington, DC with a Travel Award from the Society for Neuroscience. In 2018 Mr. Diniz applied for the PhD at Maastricht University under the sponsorship of Prof. Harry Steinbusch and Prof. Jackson Bittencourt. During his scientific trajectory Mr. Diniz has focused on understanding the interactions between lactation and hypothalamic neuropeptides, while also branching his research into other areas, such as neurogenesis, modes of neuronal communication and ligand-receptor interactions. 


\section{List of Publications}

1 The weaning period promotes alterations in the orexin neuronal population of rats in a sucklingdependent manner

Diniz GB, Candido PL, Klein MO, Alvisi RD, Presse F, Nahon J-L, Felicio LF and Bittencourt JC

Brain Structure and Function [ePub ahead of print] (2018)

DOI: 10.1007/s00429-018-1723-0

2 Morphological and cellular characterization of the fetal canine (Canis lupus familiaris) subventricular zone, rostral migratory stream and olfactory bulb

Orechio D, Aguiar BA, Diniz GB, Bittencourt JC, Haemmerle CAS, Watanabe I-S, Miglino MA and Castelucci P

The Anatomical Record (2018)

DOI: 10.1002/ar.23855

\section{Neuroanatomical Structure of the MCH System}

Bittencourt JC and Diniz GB

In: Pandi-Perumal S., Torterolo P., Monti J. (eds) Melanin-Concentrating Hormone and Sleep. Springer, Cham, 2018. [ISBN:

978-3-319-75764-3]

DOI: 10.1007/978-3-319-75765-0_1

4 Litter size determines the number of melanin-concentrating hormone neurons in the medial preoptic area of lactating dams

Ferreira JCG, Duarte JCG, Diniz GB and Bittencourt JC

Physiology and Behavior 181:75-79 (2017)

DOI: 10.1016/j.physbeh.2017.08.028

5 Anatomical organization of urocortin 3-synthesizing neurons and immunoreactive terminals in the central nervous system of non-human primates [Sapajus spp.]

Battagello DS, Diniz GB, Candido PL, Da-Silva JM, De-Oliveira AR, Torres-da-Silva KR, Lotfi CF, De-Oliveira JA, Sita LV, Casatti CA,

Lovejoy DA and Bittencourt JC

Frontiers in Neuroanatomy 11:1-17 (2017)

DOI: 10.3389/fnana.2017.00057

6 The melanin-concentrating hormone as an integrative peptide driving motivated behaviors

Diniz GB and Bittencourt JC

Frontiers in Systems Neuroscience 11:1-32 (2017)

DOI: $10.3389 /$ fnsys.2017.00032

7 Suckling-induced Fos activation and melanin-concentrating hormone immunoreactivity during late lactation

Alvisi RD', Diniz GB'1, Da-Silva JM, Bittencourt JC and Felicio LF

Life Sciences 148:241-246 (2016)

DOI: $10.1016 / j . l f s .2016 .02 .038$

8 Effect of intrahippocampal administration of anti-melanin-concentrating hormone on spatial foodseeking behavior in rats 
Sita LV, Diniz GB, Canteras NS, Xavier GF and Bittencourt JC Peptides 76:130-138 (2016)

DOI: 10.1016/j.peptides.2015.12.007

\section{The centrally-projecting Edinger-Westphal nucleus - I: Efferents in the rat brain}

Dos-Santos-Júnior ED, Da-Silva AV, Da-Silva KRT, Haemmerle CAS, Batagello DS, Da-Silva JM, Lima LB, Da-Silva RJ, Diniz GB, Sita LV, Elias CF and Bittencourt JC

Journal of Chemical Neuroanatomy 68:22-38 (2015)

DOI: 10.1016/j.jchemneu.2015.07.002 



\section{Appendix E}

Acknowledgements 



\section{Acknowledgements}

I would like to acknowledge all those who contributed to this work, be through financial, infrastructural or emotional support. Although they are too many to be named individually, I hope those who have touched this Dissertation and my life will feel reassured of my everlasting gratitude.

First and foremost, I would like to thank my supervisor, Prof. Dr. Jackson Cioni Bittencourt, for his guidance and friendship for the last 6 years. I consider myself very lucky to have met Prof. Bittencourt in the very early years of my professional development, as he has trusted and invested in my career from the moment I started in his lab. I would also like to thank my second supervisor, Prof. Dr. Jean-Louis Nahon, for taking me under his wing while I was in France and contributing so much to my personal and professional growth while I was living abroad. Working at his lab in the beautiful Sophia-Antipolis has been one of the best experiences I had in my life. Finally, I would like to thank Prof. Dr. Harry W. M. Steinbusch for giving me the once-in-a-lifetime opportunity of presenting my Dissertation for consideration at Maastricht University, something I would have never dreamed of when I was just starting in science. Just by glancing at the list of mentors I encountered in my life, one can readily see how lucky I have been so far.

My labmates have always been, and continue to be, an incomparable source of help, learning and fun. A special thanks should be given to Prof. Dr. Luciane V. Sita for her always useful comments and suggestions, and for the delightful time we spend together at lunch. To Dr. Daniella S. Battagello I owe an eternal debt of gratitude for her help in each and everything I do at the lab, fruit of her unbound desire to help others flourish. And, of course, I want to give a special thanks to all my colleagues and collaborators that helped make this Dissertation a possibility: Dr. Jozélia G. P. Ferreira, Ms. Jéssica C. G. Duarte, Mr. Renato D. Alvisi, Mr. Paulo L. Candido and Dr. Marianne O. Klein.

None of the above would be a reality without financial support from several sources. I am particular grateful to the University of São Paulo, my Alma Mater, for its continuous support for my education and the awards received. Those awards opened the doors of the World to me. I must also thank the São Paulo Research Foundation (FAPESP) for financially supporting each step of my career so far, as well as the Brazilian Coordination for the Improvement of Higher Education Personnel (CAPES) and the Brazilian National Council for Scientific and Technological Development (CNPq). Finally, I must wholeheartedly thank the Society for Neuroscience (SfN), the International Brain Research Organization (IBRO) and the Grass Foundation for the gift of participating of the Latin American Training Program (LATP) and attending the SfN Conference in Washington DC.

Last, but not least, I must thank all those who supported me on a personal level. Chief among those are my parents, Adaberio and Isaltina, for their unconditional love and support. My interests in science and knowledge were born at hearth. To my dear friends João Paulo, Gabriel, Daniel and Ricardo, I express my gratitude for all the laughs and the good times we spent together in the last decade or so. Even at the most troubling times I've encountered, they never ceased to support me. Finally, I want to thank Robert W. Galm for all the support and companionship he has provided me in the past few years. 
